\title{
ON THE LENGTH OF THE LONGEST COMMON SUBSEQUENCE OF TWO INDEPENDENT MALLOWS PERMUTATIONS
}

\author{
by
}

Ke Jin

A dissertation submitted to the Faculty of the University of Delaware in partial fulfillment of the requirements for the degree of Doctor of Philosophy in Applied Mathematics

Spring 2017

(C) 2017 Ke Jin

All Rights Reserved 


\section{ON THE LENGTH OF THE LONGEST COMMON SUBSEQUENCE OF TWO INDEPENDENT MALLOWS PERMUTATIONS}

Ke Jin

Approved:

Louis Rossi, Ph.D.

Chair of the Department of Mathematical Science

Approved:

George Watson, Ph.D.

Dean of the College of Arts \& Sciences

Approved:

Ann L. Ardis, Ph.D.

Senior Vice Provost for Graduate and Professional Education 
I certify that I have read this dissertation and that in my opinion it meets the academic and professional standard required by the University as a dissertation for the degree of Doctor of Philosophy.

Signed:

Nayantara Bhatnagar, Ph.D.

Professor in charge of dissertation

I certify that I have read this dissertation and that in my opinion it meets the academic and professional standard required by the University as a dissertation for the degree of Doctor of Philosophy.

Signed:

Mokshay Madiman, Ph.D.

Member of dissertation committee

I certify that I have read this dissertation and that in my opinion it meets the academic and professional standard required by the University as a dissertation for the degree of Doctor of Philosophy.

Signed:

Douglas Rizzolo, Ph.D.

Member of dissertation committee

I certify that I have read this dissertation and that in my opinion it meets the academic and professional standard required by the University as a dissertation for the degree of Doctor of Philosophy.

Signed:

Shannon Starr, Ph.D.

Member of dissertation committee 


\section{ACKNOWLEDGEMENTS}

I would like to thank my advisor Nayantara Bhatnagar for her guidance and encouragement during my Phd studies. She is gifted at explaining abstract concept in an intuitive way which benefits me a lot. I am also amazed by her ability to insightfully relate some known results to the problem being investigated. The completion of this dissertation would not be possible without her guidance.

I would like to thank the admission committee who give me the chance to pursue Phd in mathematics at University of Delaware. I also want to thank the faculty of the Department of Mathematical Science. It has been a fruitful experience attending the lectures offered by the department. The instructors are all very nice and helpful.

I would like to thank Shuying Sun, without whom life would not be as colorful as it has been.

I would like to thank my parents Keqin Jin and Guiying Zhou for their faith in me as well as their tolerance of my foolishness. This dissertation is dedicated to my parents. 


\section{TABLE OF CONTENTS}

LIST OF FIGURES . . . . . . . . . . . . . . . . . . vii

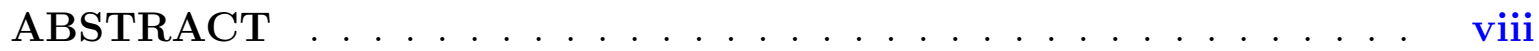

\section{Chapter}

1 INTRODUCTION . . . . . . . . . . . . . . . . . 1

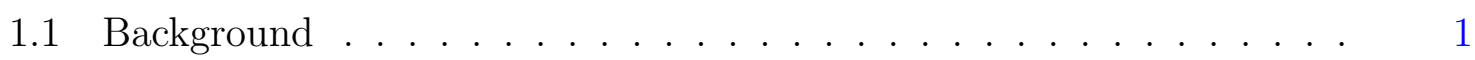

1.1.1 Longest Common Subsequence Problem . . . . . . . . . . . . 1

1.1.2 Mallows Measure on Symmetric Group $S_{n} \ldots \ldots . . . . .2$

1.1.3 Limiting Laws for the LIS of Mallows Permutation . . . . . . 3

1.2 Main Results ........................ 5

1.2.1 Convergence of Empirical Measure . . . . . . . . . . . . 5

1.2.2 Weak Law of Large Numbers for the LCS . . . . . . . . . . . . . 7

1.2.3 Central Limit Theorem for the LCS . . . . . . . . . . . . . . 9

2 CONVERGENCE OF EMPIRICAL MEASURES . . . . . . . . . 11

2.1 Two Key Lemmas . . . . . . . . . . . . . . . . . . . . . 11

2.1.1 Preliminaries ............................. 12

2.1.2 One dimension analog of Theorem 1.2.1 . . . . . . . . . . 15

2.1.3 Proof of Lemma 2.1.1 and Lemma 2.1.2 . . . . . . . . . . . 29

2.2 Proof of Theorem 1 . . . . . . . . . . . . . . . . . . . . . . . . . 42

2.3 Convergence of the Empirical Measure . . . . . . . . . . . . . 44

3 WEAK LAW OF LARGE NUMBERS WHEN AT LEAST ONE PERMUTATION IS CLOSE TO UNIFORMLY RANDOM . . . . 50

3.1 Reduction LCS problem to LIS problem . . . . . . . . . . . . 50 
3.2 Weak Bruhat Order . . . . . . . . . . . . . . . . . 52

3.3 Proof of WLLN when both $\beta$ and $\gamma$ are finite . . . . . . . . 62

3.4 More Couplings of Mallows Permutations . . . . . . . . . . . . . 87

3.5 Proof of WLLN when only $\beta$ is finite . . . . . . . . . . . . 92

3.6 Proof of Lemma 3.3.1 and Lemma 3.3.2 . . . . . . . . . . . 97

4 WEAK LAW OF LARGE NUMBERS WHEN NEITHER PERMUTATION IS CLOSE TO UNIFORMLY RANDOM . . . . 113

$4.1 \quad$ Mallows process . . . . . . . . . . . . . . . . . . . . . 113

4.2 Block decomposition . . . . . . . . . . . . . . . . . . . 116

4.3 Proof of Lemma $4.2 .2 \ldots \ldots \ldots$

5 CENTRAL LIMIT THEOREM FOR THE LCS . . . . . . . . . 130

5.1 Bound LCS via Regenerative Process . . . . . . . . . . . . . . . 130

5.1.1 Constructing Mallows Permutations . . . . . . . . . . . . . 130

5.1.2 The Regenerative Process Representation . . . . . . . . . . . 131

5.2 Renewal Time Estimate and the CLT . . . . . . . . . . . . . . 132

BIBLIOGRAPHY . . . . . . . . . . . . . . . . 139 


\section{LIST OF FIGURES}

$2.1 \pi=(4,1,7,3,6,2,5) \ldots \ldots \ldots \ldots$

$2.2 \quad \pi^{(4)}=(3,1,6,5,2,4) \ldots \ldots \ldots \ldots \ldots$

2.3 Row insertion ........................ . . . 14

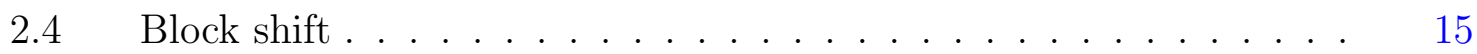




\begin{abstract}
The Mallows measure is a probability measure on $S_{n}$ where the probability of a permutation $\pi$ is proportional to $q^{l(\pi)}$ with $q>0$ being a parameter and $l(\pi)$ the number of inversions in $\pi$. We prove three weak laws of large numbers and a central limit theorem for the length of the longest common subsequences of two independent permutations drawn from the Mallows measure for different regimes of the parameter $q$.
\end{abstract}




\section{Chapter 1 \\ INTRODUCTION}

\section{$1.1 \quad$ Background}

\subsubsection{Longest Common Subsequence Problem}

Definition 1.1.1. Given two strings $s, t$, let $s_{i}$ denote the $i$-th character in $s$. The length of the longest common subsequence of $s$ and $t$ is defined by,

$$
\begin{array}{r}
\operatorname{LCS}(s, t):=\max \left(m: \exists i_{1}<\cdots<i_{m} \text { and } j_{1}<\cdots<j_{m}\right. \\
\text { such that } \left.s_{i_{k}}=t_{j_{k}} \text { for all } k \in[m]\right) .
\end{array}
$$

The length of the longest common subsequence of two permutations is defined similarly as follows

$$
\begin{aligned}
& \operatorname{LCS}(\pi, \tau):=\max \left(m: \exists i_{1}<\cdots<i_{m} \text { and } j_{1}<\cdots<j_{m}\right. \\
& \text { such that } \pi\left(i_{k}\right)\left.=\tau\left(j_{k}\right) \text { for all } k \in[m]\right) .
\end{aligned}
$$

The longest common subsequence(LCS) problem is a classical problem which has application in fields such as molecular biology (see, e.g., [23]), data comparison and software version control. Most previous works on the LCS problem are focused on the case when the strings are generated uniformly at random from a given alphabet. Notably, Chvátal and Sankoff [7] proved that the expected length of the LCS of two random $k$-ary sequences of length $n$ when normalized by $n$ converges to a constant $\gamma_{k}$. Since then, various endeavors $[11,9,10,19]$ have been made to determine the value of $\gamma_{k}$. The exact values of $\gamma_{k}$ are still unknown. The known lower and upper bounds [19] for $\gamma_{2}$ are

$$
0.788071<\gamma_{2}<0.826280
$$


In contrast to the LCS of two random strings, the LCS of two permutations is well connected to the longest increasing subsequence(LIS) problem (cf. Proposition 3.1 in [16]). This can be seen from the following two facts,

- For any $\pi \in S_{n}$, the length of the LCS of $\pi$ and the identity in $S_{n}$ is equal to the length of the LIS of $\pi$.

- For any $\pi, \tau \in S_{n}$, the length of the LCS of $\pi$ and $\tau$ is equal to the length of the LCS of $\tau^{-1} \circ \pi$ and the identity in $S_{n}$.

From the above two properties, it is easily seen that, if $\pi, \tau$ are independent and either $\pi$ or $\tau$ is uniformly distributed on $S_{n}$ the length of the LCS of $\pi$ and $\tau$ has the same distribution as the length of the LIS of a uniformly random permutation. The length of the LIS of a uniformly random permutation has been well studied with major contributions from Hammersley [15], Logan and Shepp [18], Vershik and Kerov [17] and culminating with the groundbreaking work of Baik, Deift and Johansson [4] who prove that, under proper scaling, the length of the LIS converges to the Tracy-Widom distribution. Therefore, the length of the LCS of two independent permutations is only of interest when both permutations are non-uniformly distributed.

\subsubsection{Mallows Measure on Symmetric Group $S_{n}$}

Definition 1.1.2. Given $\pi \in S_{n}$, the inversion set of $\pi$ is defined by

$$
\operatorname{Inv}(\pi):=\{(i, j): 1 \leq i<j \leq n \text { and } \pi(i)>\pi(j)\}
$$

and the inversion number of $\pi$, denoted by $l(\pi)$, is defined to be the cardinality of $\operatorname{Inv}(\pi)$.

The Mallows measure on $S_{n}$ is introduced by Mallows in [20] to study nonuniform distributions on permutations. For $q>0$, the $(n, q)$ - Mallows measure on $S_{n}$ is given by

$$
\mu_{n, q}(\pi):=\frac{q^{l(\pi)}}{Z_{n, q}}, \quad \text { where } \quad Z_{n, q}=\prod_{i=1}^{n} \frac{1-q^{i}}{1-q}
$$


Here $Z_{n, q}$ is the normalizing constant, which has an explicit form (see, e.g., [25] Corollary 1.3.13). In other words, under the Mallows measure with parameter $q>0$, the probability of a permutation $\pi$ is proportional to $q^{l(\pi)}$.

Mallows measure has been used in modeling ranked and partially ranked data (see, e.g., [8, 13, 21]). In [8], Critchlow provides several examples where Mallows model gives a good fit to ranking data.

We list a couple of properties of Mallows permutation. The proofs of the following lemmas can be found in Section 2 in [6].

Definition 1.1.3. Given $\pi \in S_{n}$, let $\pi^{-1}$ denote the inverse of $\pi$ in $S_{n}$ and $\pi^{r}$ denote the reversal of $\pi$ which is defined by $\pi^{r}(i)=\pi(n+1-i)$. Let $\boldsymbol{a}=\left(a_{1}, \ldots, a_{k}\right)$ be an increasing sequence of indices in $[n]$. Define $\pi(\boldsymbol{a}):=\left(\pi\left(a_{1}\right), \ldots, \pi\left(a_{k}\right)\right)$. Let $\pi_{\boldsymbol{a}}$ denote

the induced permutation of $\pi(\boldsymbol{a})$ in $S_{k}$ where $\pi_{\boldsymbol{a}}(i)=j$ if $\pi\left(a_{i}\right)$ is the $j$-th smallest term in $\pi(\boldsymbol{a})$.

Lemma 1.1.4. For any $q>0$, if $\pi \sim \mu_{n, q}$ then $\pi^{r} \sim \mu_{n, 1 / q}$ and $\pi^{-1} \sim \mu_{n, q}$.

Lemma 1.1.5. Let $\boldsymbol{a}=\left(a_{1}, \ldots, a_{k}\right)$ and $\boldsymbol{b}=\left(b_{1}, \ldots, b_{l}\right)$ be two increasing sequences of indices in $[n]$ such that $a_{k}<b_{1}$. If $\pi \sim \mu_{n, q}$, then $\pi_{\boldsymbol{a}}$ and $\pi(\boldsymbol{b})$ are independent and $\pi(\boldsymbol{a})$ and $\pi_{\boldsymbol{b}}$ are independent.

Lemma 1.1.6. Let $\boldsymbol{I}=(i, i+1, \ldots, i+m-1) \subset[n]$ be a sequence of consecutive indices. If $\pi \sim \mu_{n, q}$, then $\pi_{\boldsymbol{I}} \sim \mu_{m, q}$ and $\pi_{\pi^{-1}(\boldsymbol{I})} \sim \mu_{m, q}$. Moreover, conditioned on $\pi^{-1}(\boldsymbol{I})=E \subset[n]$, we still have $\pi_{E} \sim \mu_{m, q}$.

\subsubsection{Limiting Laws for the LIS of Mallows Permutation}

In this subsection, we introduce several limiting results about the length of the longest increasing subseqence(LIS) of Mallows permutation. Due to the intimate connection between the increasing subsequence and common subsequence of two permutations, we have adapted many ideas from the proofs of the following results in establishing the limiting laws of the LCS for Mallows permutation. 
In [22], Mueller and Starr prove the following weak law of large numbers for the LIS of Mallows permutation where the parameter $q$ is a function of $n$ and $n(1-q)$ has limit in $\mathbb{R}$ as $n \rightarrow \infty$.

Theorem 1.1.7. Suppose $\left\{q_{n}\right\}_{n=1}^{\infty}$ is a sequence such that $\lim _{n \rightarrow \infty} n\left(1-q_{n}\right)=\beta$ with $\beta \in \mathbb{R}$. Then

$$
\lim _{n \rightarrow \infty} \mu_{n, q_{n}}\left(\left|\frac{L I S(\pi)}{\sqrt{n}}-2 \kappa(\beta)\right|>\epsilon\right)=0
$$

where

$$
\kappa(\beta)= \begin{cases}\beta^{-1 / 2} \sinh ^{-1}\left(\sqrt{e^{\beta}-1}\right) & \text { for } \beta>0 ; \\ 1 & \text { for } \beta=0 ; \\ |\beta|^{-1 / 2} \sin ^{-1}\left(\sqrt{1-e^{\beta}}\right) & \text { for } \beta<0 .\end{cases}
$$

Their proof of Theorem 1.1.7, which makes use of the techniques developed by Deuschel and Zeitouni in [12], is based on a coupling argument and the following classic result of the LIS of the uniformly random permutations (cf. [17, 18])

Theorem 1.1.8. Let $\lambda_{n}$ denote the uniform probability measure on $S_{n}$. Then, for any $\epsilon>0$

$$
\lim _{n \rightarrow \infty} \lambda_{n}\left(\left|\frac{\operatorname{LIS}(\pi)}{\sqrt{n}}-2\right|<\epsilon\right)=1
$$

In [6], Bhatnagar and Peled prove the following $L_{p}$ convergence of the LIS of Mallows permutation in the regime where $q_{n} \rightarrow 1$ in a way such that $n\left(1-q_{n}\right) \rightarrow \infty$.

Theorem 1.1.9. Let $\left\{q_{n}\right\}_{n=1}^{\infty}$ be a sequence such that

$$
\lim _{n \rightarrow \infty} q_{n}=1 \quad \text { and } \quad \lim _{n \rightarrow \infty} n\left(1-q_{n}\right)=\infty
$$

Suppose $\pi_{n} \sim \mu_{n, q_{n}}$. Then, for any $p>0$,

$$
\frac{\operatorname{LIS}\left(\pi_{n}\right)}{n \sqrt{1-q_{n}}} \stackrel{L_{p}}{\longrightarrow} 1 .
$$

For the regime where the parameter $q$ is fixed with $0<q<1$, Basu and Bhatnagar show the following central limit theorem of the LIS of Mallows permutation in $[5]$. 
Theorem 1.1.10. Fix $0<q<1$. Suppose $\pi_{n} \sim \mu_{n, q}$. Then there exist constants $\sigma=\sigma(q)>0$ and $a=a(q)>0$ such that

$$
\frac{\operatorname{LIS}\left(\pi_{n}\right)-a n}{\sigma \sqrt{n}} \stackrel{d}{\longrightarrow} \mathcal{N}(0,1)
$$

as $n \rightarrow \infty$, where $\mathcal{N}(0,1)$ denotes the standard normal distribution.

\subsection{Main Results}

\subsubsection{Convergence of Empirical Measure}

Our first two theorems are about the convergence of the empirical measure of Mallows permutation. They are inspired by and are direct analogs to the following theorem proved by Starr in [26]. The reason to prove them is that they give us an estimate about the number of points which fall in a given rectangle, where those points are defined by two Mallows permutations. Specifically, Theorem 2 plays the same role in the proof of Theorem 3 as Theorem 1.2.1 in the proof of Theorem 1.1.7.

Theorem 1.2.1 (S.Starr). Suppose that $\left(q_{n}\right)_{n=1}^{\infty}$ is a sequence such that the limit $\beta=$ $\lim _{n \rightarrow \infty} n\left(1-q_{n}\right)$ exists. For any $\epsilon>0$ and any continuous function $f:[0,1] \times[0,1] \rightarrow$ $\mathbb{R}$

$$
\lim _{n \rightarrow \infty} \mu_{n, q_{n}}\left(\left|\frac{1}{n} \sum_{i=1}^{n} f\left(\frac{i}{n}, \frac{\pi(i)}{n}\right)-\int_{[0,1] \times[0,1]} f(x, y) u(x, y, \beta) d x d y\right|>\epsilon\right)=0
$$

where

$$
u(x, y, \beta):=\frac{(\beta / 2) \sinh (\beta / 2)}{\left(e^{\beta / 4} \cosh (\beta[x-y] / 2)-e^{-\beta / 4} \cosh (\beta[x+y-1] / 2)\right)^{2}}
$$

if $\beta \neq 0$, and $u(x, y, 0):=1$.

Our first result is about the distribution of $\frac{\pi(i)}{n}$ in the regime of the Mallows measure where $\lim _{n \rightarrow \infty} n\left(1-q_{n}\right)$ exists. It says that the distribution of $\frac{\pi(i)}{n}$ approaches towards the measure with density $u\left(\frac{i}{n}, y, \beta\right)$ uniformly on $i \in[n]$. Here the 'approach' is in the sense that, given any continuous function $f$ on $[0,1]$, the expectation of $f$

with respect to the empirical measure of $\frac{\pi(i)}{n}$ converges uniformly to the expectation of 
$f$ with respect to the probability with density $u\left(\frac{i}{n}, y, \beta\right)$. Moreover, the covariance of $f\left(\frac{\pi(i)}{n}\right)$ and $f\left(\frac{\pi(j)}{n}\right)$ converges to 0 uniformly on all pairs $(i, j)$ where $i \neq j$.

Theorem 1. Suppose that $\left\{q_{n}\right\}_{n=1}^{\infty}$ is a sequence such that $\lim _{n \rightarrow \infty} n\left(1-q_{n}\right)=\beta \in \mathbb{R}$. For any continuous function $f:[0,1] \longrightarrow \mathbb{R}$, we have

$$
\lim _{n \rightarrow \infty} \max _{i \in[n]}\left|\mu_{n, q_{n}}\left(f\left(\frac{\pi(i)}{n}\right)\right)-\int_{0}^{1} f(y) \cdot u\left(\frac{i}{n}, y, \beta\right) d y\right|=0
$$

and

$$
\lim _{n \rightarrow \infty} \max _{\substack{i \neq j \\ i, j \in[n]}}\left|\operatorname{Cov}_{n}\left(f\left(\frac{\pi(i)}{n}\right), f\left(\frac{\pi(j)}{n}\right)\right)\right|=0 .
$$

Here $u(x, y, \beta)$ is defined in (1.3), and

$$
\begin{aligned}
\operatorname{Cov}_{n}\left(f\left(\frac{\pi(i)}{n}\right), f\left(\frac{\pi(j)}{n}\right)\right) & := \\
& \mu_{n, q_{n}}\left(f\left(\frac{\pi(i)}{n}\right) f\left(\frac{\pi(j)}{n}\right)\right)-\mu_{n, q_{n}}\left(f\left(\frac{\pi(i)}{n}\right)\right) \mu_{n, q_{n}}\left(f\left(\frac{\pi(j)}{n}\right)\right) .
\end{aligned}
$$

Theorem 1 is a major step in proving the next theorem, which shows the convergence of the empirical measure defined by the product of two independent Mallows permutations.

Theorem 2. Suppose that $\left\{q_{n}\right\}_{n=1}^{\infty}$ and $\left\{q_{n}^{\prime}\right\}_{n=1}^{\infty}$ are two sequences such that $\lim _{n \rightarrow \infty} n\left(1-q_{n}\right)=\beta$ and $\lim _{n \rightarrow \infty} n\left(1-q_{n}^{\prime}\right)=\gamma$, with $\beta, \gamma \in \mathbb{R}$. Let $\mathbb{P}_{n}$ denote the probability measure on $S_{n} \times S_{n}$ such that $\mathbb{P}_{n}((\pi, \tau))=\mu_{n, q_{n}}(\pi) \cdot \mu_{n, q_{n}^{\prime}}(\tau)$, i. e. $\mathbb{P}_{n}$ is the product measure of $\mu_{n, q_{n}}$ and $\mu_{n, q_{n}^{\prime}}$. Let $\tau \circ \pi$ denote the product of $\tau$ and $\pi$ in $S_{n}$ with $\tau \circ \pi(i)=\tau(\pi(i))$. Then, for any $\epsilon>0$,

$$
\lim _{n \rightarrow \infty} \mathbb{P}_{n}\left(\left|\frac{1}{n} \sum_{i=1}^{n} f\left(\frac{i}{n}, \frac{\tau \circ \pi(i)}{n}\right)-\int_{0}^{1} \int_{0}^{1} f(x, y) \rho(x, y) d x d y\right|>\epsilon\right)=0
$$

for every continuous function $f:[0,1] \times[0,1] \rightarrow \mathbb{R}$, with

$$
\rho(x, y):=\int_{0}^{1} u(x, t, \beta) \cdot u(t, y, \gamma) d t
$$

where $u(x, y, \beta)$ is defined in (1.3). 


\subsubsection{Weak Law of Large Numbers for the LCS}

Our next result is the following weak law of large numbers for the LCS of two independent Mallows permutations.

Theorem 3. Let $B_{\nearrow}^{1}$ denote the set of nondecreasing, $C_{b}^{1}$ functions $\phi:[0,1] \rightarrow[0,1]$, with $\phi(0)=0$ and $\phi(1)=1$. Define function $J: B_{\nearrow}^{1} \rightarrow \mathbb{R}$,

$$
J(\phi):=\int_{0}^{1} \sqrt{\dot{\phi}(x) \rho(x, \phi(x))} d x, \quad \text { and } \quad \bar{J}:=\sup _{\phi \in B_{\nearrow}^{1}} J(\phi) .
$$

Here $\rho(x, y)$ is the density defined in (1.6). Under the same conditions as in Theorem 2, for any $\epsilon>0$, we have

$$
\lim _{n \rightarrow \infty} \mathbb{P}_{n}\left(\left|\frac{L C S(\pi, \tau)}{\sqrt{n}}-2 \bar{J}\right|<\epsilon\right)=1
$$

We derive the limiting constant in the special case when $\beta=\gamma$.

Corollary 3. Suppose that $\left\{q_{n}\right\}_{n=1}^{\infty}$ and $\left\{q_{n}^{\prime}\right\}_{n=1}^{\infty}$ are two sequences such that $\lim _{n \rightarrow \infty} n\left(1-q_{n}\right)=\lim _{n \rightarrow \infty} n\left(1-q_{n}^{\prime}\right)=\beta$ with $\beta \neq 0$. Then, the constant $\bar{J}$ in Theorem 3 is given by

$$
\bar{J}=\sqrt{\frac{\beta}{6 \sinh (\beta / 2)}} \cdot \int_{0}^{1} \sqrt{\cosh (\beta / 2)+2 \cosh (\beta[2 x-1] / 2)} d x .
$$

There are three main ingredients in our proof of Theorem 3. The first observation, which is proved in Corollary 3.1.4, is that the length of LCS of two permutations $\pi$ and $\tau$ is equal to the length of the longest increasing points in the collection of points

$$
\boldsymbol{z}\left(\pi^{-1}, \tau^{-1}\right):=\left\{\left(\frac{\pi^{-1}(i)}{n}, \frac{\tau^{-1}(i)}{n}\right)\right\}_{i \in[n]} .
$$

The second observation, deduced from Lemma 2.3.1, is that the number of points in $\boldsymbol{z}\left(\pi^{-1}, \tau^{-1}\right)$ contained in any fixed rectangle, when scaled by the size of the permutation, converges in probability to a constant. The third observation, proved in Lemma 3.3.4, is that the length of the longest increasing points in $\boldsymbol{z}\left(\pi^{-1}, \tau^{-1}\right)$ within a small box $R$ is close to the size of the LIS in the uniform case, i.e., it is approximately 
$2 \sqrt{\left|\boldsymbol{z}\left(\pi^{-1}, \tau^{-1}\right) \cap R\right|}$. With these results, we prove Theorem 3 following the method developed by Deuschel and Zeitouni in [12] for record lengths of i.i.d. points.

Using Theorem 3 and Theorem 1.1.7, by a coupling argument, we are able to prove the following weak law of large numbers for the LCS of two independent Mallows permutations when the parameters $q_{n}, q_{n}^{\prime}$ belong to a different regime where one of $n\left(1-q_{n}\right)$ and $n\left(1-q_{n}^{\prime}\right)$ has limit in $\mathbb{R}$ and the other diverges to infinity as $n \rightarrow \infty$.

Theorem 4. Suppose that $\left\{q_{n}\right\}_{n=1}^{\infty}$ and $\left\{q_{n}^{\prime}\right\}_{n=1}^{\infty}$ are two sequences such that $\lim _{n \rightarrow \infty} n\left(1-q_{n}\right)=\infty$, and $\lim _{n \rightarrow \infty} n\left(1-q_{n}^{\prime}\right)=\beta$ with $\beta \in \mathbb{R}$. Let $\mathbb{P}_{n}$ denote the probability measure on $S_{n} \times S_{n}$ such that $\mathbb{P}_{n}((\pi, \tau))=\mu_{n, q_{n}}(\pi) \cdot \mu_{n, q_{n}^{\prime}}(\tau)$, i.e. $\mathbb{P}_{n}$ is the product measure of $\mu_{n, q_{n}}$ and $\mu_{n, q_{n}^{\prime}}$. Then for any $\epsilon>0$, we have

$$
\lim _{n \rightarrow \infty} \mathbb{P}_{n}\left(\left|\frac{L C S(\pi, \tau)}{\sqrt{n}}-2 \kappa(\beta)\right|>\epsilon\right)=0,
$$

where

$$
\kappa(\beta)= \begin{cases}\beta^{-1 / 2} \sinh ^{-1}\left(\sqrt{e^{\beta}-1}\right) & \text { for } \beta>0 ; \\ 1 & \text { for } \beta=0 ; \\ |\beta|^{-1 / 2} \sin ^{-1}\left(\sqrt{1-e^{\beta}}\right) & \text { for } \beta<0 .\end{cases}
$$

Comparing Theorem 4 with Theorem 1.1.7, it can be seen that the LCS between $\pi$ and $\tau$ in the setting of Theorem 4 observes the same weak law of large numbers as the LIS of a Mallows permutation with a sequence of parameters which goes to 1 at the same rate as the slower one in the setting of Theorem 4. Note that $\operatorname{LCS}(i d, \tau)=\operatorname{LIS}(\tau)$, where $i d$ denote the identity in $S_{n}$. Hence, roughly speaking, Theorem 4 says that the influence of a Mallows permutation $\pi$, which is concentrated near identity, to the weak law of $\operatorname{LCS}(\pi, \tau)$ is just like that of the identity in the sense that if we replace $\pi$ by the identity, the magnitude as well as the constant of the weak law of $\operatorname{LCS}(\pi, \tau)$ do not change.

The following theorem establishes the $L_{p}$ convergence for the LCS of two independent Mallows permutations with same parameter $q_{n}$, such that $\lim _{n \rightarrow \infty} q_{n}=1$ and $\lim _{n \rightarrow \infty} n\left(1-q_{n}\right)=\infty$. 
Theorem 5. Suppose $\left\{q_{n}\right\}$ is a sequence such that

$$
\lim _{n \rightarrow \infty} q_{n}=1 \quad \text { and } \quad \lim _{n \rightarrow \infty} n\left(1-q_{n}\right)=\infty .
$$

For each $n$, define two independent random variables $\pi_{n}, \tau_{n}$ such that $\pi_{n} \sim \mu_{n, q_{n}}$ and $\tau_{n} \sim \mu_{n, q_{n}}$. Then, for any $0<p<\infty$,

$$
\frac{L C S\left(\pi_{n}, \tau_{n}\right)}{n \sqrt{1-q_{n}}} \stackrel{L_{p}}{\longrightarrow} \frac{\sqrt{6}}{3}
$$

as $n$ tends to infinity.

The proof of Theorem 5 follows the approach developed in [6], where the authors show Theorem 1.1.9.

\subsubsection{Central Limit Theorem for the LCS}

Our last result is a central limit theorem for the LCS of two independent Mallows permutations, where the parameters $0<q, q^{\prime}<1$ are fixed numbers.

Theorem 6. Given $0<q, q^{\prime}<1$, for each $n>0$ define two independent random variables $\pi_{n}, \tau_{n}$ such that $\pi_{n} \sim \mu_{n, q}$ and $\tau_{n} \sim \mu_{n, q^{\prime}}$. There exist constant $\sigma=\sigma\left(q, q^{\prime}\right)>$ 0 and $a=a\left(q, q^{\prime}\right)>0$ such that

$$
\frac{L C S\left(\pi_{n}, \tau_{n}\right)-a n}{\sigma \sqrt{n}} \stackrel{d}{\longrightarrow} \mathcal{N}(0,1)
$$

as $n \rightarrow \infty$. Here $\stackrel{d}{\longrightarrow}$ denotes convergence in distribution and $\mathcal{N}(0,1)$ denotes the standard normal distribution.

The proof of Theorem 6 is based on the approach developed in [5] in which the authors prove Theorem 1.1.10. The idea is to construct a regenerative processes such that we can bound the LCS by the sum of some i.i.d. random variables. Then the central limit theorem follows from the standard theory of regenerative processes.

The remainder of this dissertation is organized as follows. In Chapter 2, we prove the convergence of the empirical measure induced by the product of two independent Mallows permutations. In Chapter 3, we show a weak laws of large numbers for the LCS 
when at least one sequence $\left\{q_{n}\right\}$ is such that $n\left(1-q_{n}\right)$ has limit in $\mathbb{R}$. In Chapter 4 , we prove the $L_{p}$ convergence for the LCS when $q_{n}=q_{n}^{\prime}$ and $n\left(1-q_{n}\right) \rightarrow \infty$. In Chapter 5, we show the central limit theorem for the LCS when both parameters $q$ and $q^{\prime}$ are constant within $(0,1)$. The proofs in each chapter are largely independent of each other. 


\section{Chapter 2}

\section{CONVERGENCE OF EMPIRICAL MEASURES}

In this chapter, we show the convergence of empirical measures of the points defined by Mallows permutations. In [26], the author establishes Theorem 1.2.1 by making use of the mean field theory and evaluates the density of the limit distribution as the solution to an integrable PDE. We do not think this approach applies in our case, since the Hamiltonian is not of mean-field type. This chapter is organized as follows. In Section 2.1, we introduce and prove Lemma 2.1.1 and Lemma 2.1.2. In Section 2.2 and Section 2.3, we show Theorem 1 and Theorem 2 respectively using the two lemmas established in Section 2.1.

\subsection{Two Key Lemmas}

In this section we introduce the following two lemmas which play a key role in proving the main theorems. The proofs presented in this section are largely independent of the following sections. With these two lemmas in mind, readers can go through the proofs of Theorem 1 and Theorem 2 without trouble.

Lemma 2.1.1. Suppose $A=\left[y_{1}, y_{2}\right] \subset[0,1]$. For any $\beta \in \mathbb{R}$ and any sequence $\left\{q_{n}\right\}$ such that $q_{n}>0$ and $\lim _{n \rightarrow \infty} n\left(1-q_{n}\right)=\beta$,

$$
\lim _{n \rightarrow \infty} \max _{i \in[n]}\left|\mu_{n, q_{n}}\left(\mathbb{1}_{A}\left(\frac{\pi(i)}{n}\right)\right)-\int_{y_{1}}^{y_{2}} u\left(\frac{i}{n}, y, \beta\right) d y\right|=0 .
$$

Lemma 2.1.2. Suppose $A=\left[y_{1}, y_{2}\right] \subset[0,1]$ and $B=\left[y_{3}, y_{4}\right] \subset[0,1]$. Given $\beta \in \mathbb{R}$ and any sequence $\left\{q_{n}\right\}$ such that $q_{n}>0$ and $\lim _{n \rightarrow \infty} n\left(1-q_{n}\right)=\beta$, define

$$
\operatorname{Cov}_{n}\left(\mathbb{1}_{A}\left(\frac{\pi(i)}{n}\right), \mathbb{1}_{B}\left(\frac{\pi(j)}{n}\right)\right):=
$$




$$
\mu_{n, q_{n}}\left(\mathbb{1}_{A}\left(\frac{\pi(i)}{n}\right) \mathbb{1}_{B}\left(\frac{\pi(j)}{n}\right)\right)-\mu_{n, q_{n}}\left(\mathbb{1}_{A}\left(\frac{\pi(i)}{n}\right)\right) \mu_{n, q_{n}}\left(\mathbb{1}_{B}\left(\frac{\pi(j)}{n}\right)\right)
$$

Then, we have

$$
\lim _{n \rightarrow \infty} \max _{\substack{i \neq j \\ i, j \in[n]}}\left|\operatorname{Cov}_{n}\left(\mathbb{1}_{A}\left(\frac{\pi(i)}{n}\right), \mathbb{1}_{B}\left(\frac{\pi(j)}{n}\right)\right)\right|=0 .
$$

Lemma 2.1.1 states that in the regime of the Mallows measure where $n\left(1-q_{n}\right)$ has a limit in $\mathbb{R}$, the probability of $\frac{\pi(i)}{n}$ falling in an arbitrary interval converges to a constant uniformly for $i \in[n]$. Lemma 2.1.2 states that the covariance of $\mathbb{1}_{A}\left(\frac{\pi(i)}{n}\right)$ and $\mathbb{1}_{B}\left(\frac{\pi(j)}{n}\right)$ converges to 0 uniformly on all those pairs such that $i \neq j$.

The proofs of these two lemmas involve some computations which utilize Theorem 1.2.1 and properties of the Mallows measure. It may be the case that more general tools could be used to establish the uniform convergence of the distribution of $\frac{\pi(i)}{n}$ as well as $\operatorname{Cov}_{n}\left(\frac{\pi(i)}{n}, \frac{\pi(j)}{n}\right)$.

\subsubsection{Preliminaries}

Let $\mu$ be a probability measure on the Borel $\sigma$-field $\mathcal{B}_{\Sigma}$. We use the convention that $\mu(f):=\int_{\Sigma} f d \mu$, for any measurable function $f$. For any $\pi \in S_{n}$, let $L_{\pi}$ denote the empirical measure induced by $\pi$, that is,

$$
L_{\pi}(R):=\frac{1}{n} \sum_{i=1}^{n} \mathbb{1}_{R}\left(\frac{i}{n}, \frac{\pi(i)}{n}\right),
$$

for any $R \in \mathcal{B}_{[0,1] \times[0,1]}$. Here $\mathbb{1}_{R}(x, y)$ denotes the indicator function of $R$. Hence, for any measurable function $f$,

$$
L_{\pi}(f)=\frac{1}{n} \sum_{i=1}^{n} f\left(\frac{i}{n}, \frac{\pi(i)}{n}\right) .
$$

For any $\pi \in S_{n}$, let $\boldsymbol{z}(\pi):=\left\{\left(\frac{i}{n}, \frac{\pi(i)}{n}\right)\right\}_{i \in[n]}$ denote the set of $n$ points in $[0,1] \times$ $[0,1]$ defined by $\pi$. Conversely, for any $n$ points $V:=\left\{\left(x_{i}, y_{i}\right)\right\}_{i \in[n]}$ such that $i \neq j$ implies $x_{i} \neq x_{j}$ and $y_{i} \neq y_{j}$, we can define a permutation $\pi \in S_{n}$ as follows. Without loss of generality, assuming $x_{1}<\cdots<x_{n}$, define

$$
\pi(i):=\left|\left\{j \in[n]: y_{j} \leq y_{i}\right\}\right| .
$$


We will use $\Phi(V)$ to denote the permutation induced by $V$ as above. Similarly, we define the number of inversions of a collection points as follows,

$$
l(V):=\mid\left\{(i, j):\left(x_{i}-x_{j}\right)\left(y_{i}-y_{j}\right)<0 \text { and } i<j\right\} \mid .
$$

Note that the definition of the number of inversions of a collection of points is consistent with the definition of the number of inversions of permutation in the sense that, for any $\pi \in S_{n}$,

$$
l(\pi)=l(\boldsymbol{z}(\pi)) \quad \text { and } \quad l(V)=l(\Phi(V)) .
$$

Definition 2.1.3. For any $\pi \in S_{n}$ and $i \in[n]$, define

$$
\pi^{(i)}:=\Phi\left(\left\{\left(\frac{j}{n}, \frac{\pi(j)}{n}\right): j \neq i\right\}\right) \quad \text { and } \quad Q(\pi, i):=\left\{\tau \in S_{n}: \tau^{(i)}=\pi^{(i)}\right\} .
$$

In other words, $\pi^{(i)}$ denotes the permutation in $S_{n-1}$ which is induced from $\pi$ at those indices other than $i$, and $Q(\pi, i)$ contains those permutations in $S_{n}$ each of which has the same relative ordering as $\pi$ at those indices other than $i$.

The definition above is best understood when we represent a permutation by a grid of tiles. Specifically, for any $\pi \in S_{n}$, define an $n \times n$ grid of tiles such that the tile at $j$-th row and $i$-th column is black if only if $\pi(i)=j$. Here we index the row number from bottom to top, i.e. the bottom row is indexed as the first row. For example, the grid representations of $\pi=(4,1,7,3,6,2,5)$ and $\pi^{(4)}=(3,1,6,5,2,4)$ are shown in the following figures.

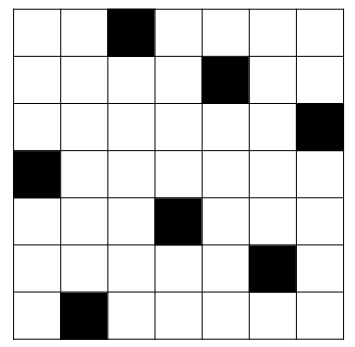

Figure 2.1: $\pi=(4,1,7,3,6,2,5)$

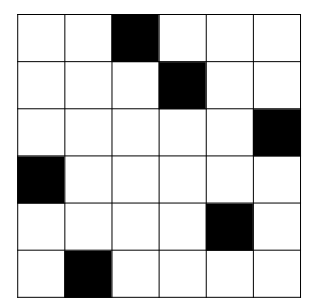

Figure 2.2: $\pi^{(4)}=(3,1,6,5,2,4)$

Note that the grid representation of $\pi^{(i)}$ can be easily obtained by deleting the $i$-th column and $\pi(i)$-th row from the grid of $\pi$. Also, the grid representations of those 
permutations other than $\pi$ in $Q(\pi, i)$ can be obtained by removing and reinserting the $\pi(i)$-th row into the grid of $\pi$. For example, it can be easily verified that $\tau=$ $(3,1,7,6,5,2,4) \in Q(\pi, 4)$. The grid representation of $\tau$ can be obtained by removing the third row from the grid of $\pi$ and reinserting it between the sixth row and seventh row of the grid of $\pi$ (see Figure 2.3).
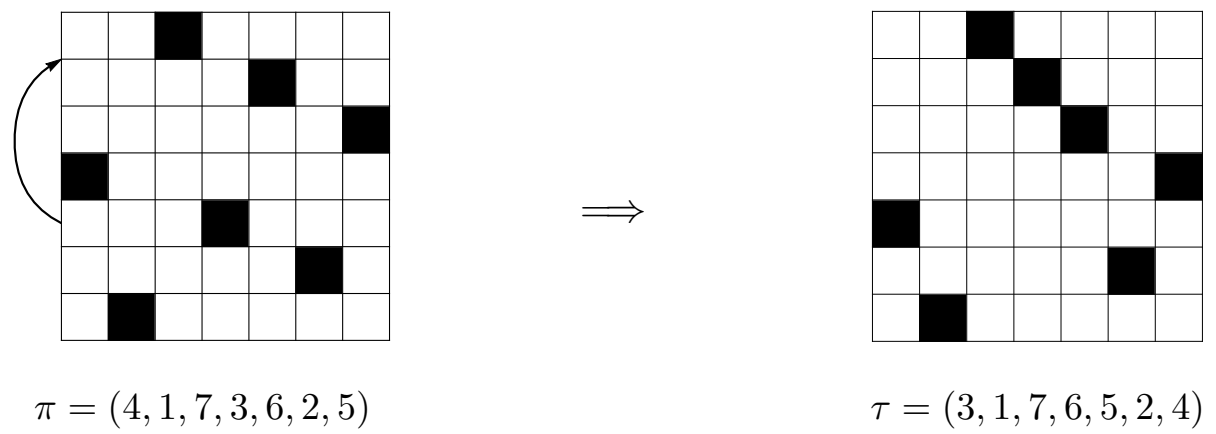

Row insertion

From this definition, it can be seen that $|Q(\pi, i)|=n$ for any $\pi \in S_{n}$. Also, for any $\pi, \tau \in S_{n}$, we have either $Q(\pi, i)=Q(\tau, i)$ or $Q(\pi, i) \cap Q(\tau, i)=\varnothing$.

Proposition 2.1.4. For any $\pi, \tau \in Q(\pi, i)$, with $\pi(i)=j<k=\tau(i)$, it holds that

$$
\begin{aligned}
l(\tau)-l(\pi) & =|\{t>i: j+1 \leq \pi(t) \leq k\}|-|\{t<i: j+1 \leq \pi(t) \leq k\}| \\
& =|\{t>i: j \leq \tau(t) \leq k-1\}|-|\{t<i: j \leq \tau(t) \leq k-1\}| .
\end{aligned}
$$

Proof. This result can be easily seen from the grid representations of $\pi$ and $\tau$. Note that an inversion in a permutation corresponds to a pair of black tiles such that one tile is located to the southeast of the other. Hence, by the discussion above, we only need to count the change of the number of those pairs when we reinsert the $j$-th row of $\pi$ 's grid to get the grid form of $\tau$. Specifically, we only need to consider those pairs which contain the black tile on the $i$-th column.

Taking the same example above, $l(\tau)-l(\pi)$ is equal to the difference of the number of black tiles within the rectangles $A$ and $B$ (see Figure 2.4). This is because, each of those black tiles in rectangle $A$ forms an inversion with the black tile in the 


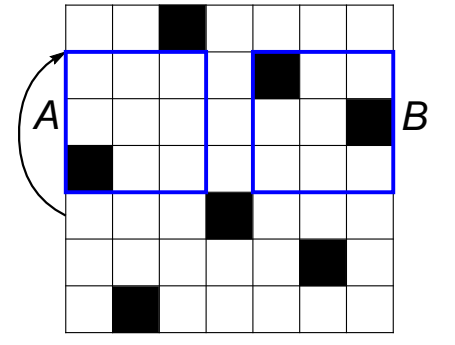

$\pi=(4,1,7,3,6,2,5)$

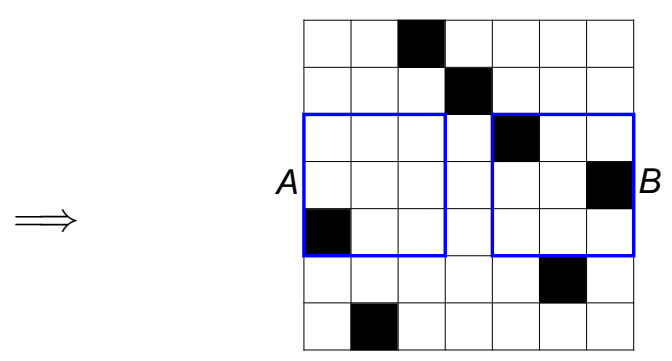

$\tau=(3,1,7,6,5,2,4)$

Block shift

fourth column in the grid representation of $\pi$ but not in that of $\tau$, whereas the opposite holds for those black tiles in the rectangle $B$.

\subsubsection{One dimension analog of Theorem 1.2.1}

The following lemma is the one dimensional analog of Theorem 1.2.1. It says that, in the regime of Mallows measure where $\lim _{n \rightarrow \infty} n\left(1-q_{n}\right)$ exists, the distribution of $\frac{\pi\left(a_{n}\right)}{n}$ converges in distribution to a probability measure with explicit density, where $\left\{a_{n}\right\}$ is a sequence of indices such that $\lim _{n \rightarrow \infty} \frac{a_{n}}{n}$ exists.

Lemma 2.1.5. Suppose that $\left\{q_{n}\right\}_{n=1}^{\infty}$ is a sequence such that $\lim _{n \rightarrow \infty} n\left(1-q_{n}\right)=\beta \in \mathbb{R}$, and $\left\{a_{n}\right\}$ is a sequence such that $\lim _{n \rightarrow \infty} \frac{a_{n}}{n}=a$, where $a \in[0,1]$ and $a_{n} \in[n]$. Then,

$$
\mu_{n, q_{n}}\left(\frac{\pi\left(a_{n}\right)}{n} \in(\cdot)\right) \stackrel{d}{\longrightarrow} v .
$$

Here $\stackrel{d}{\longrightarrow}$ denotes convergence in distribution and $v$ is the probability measure on $[0,1]$ with density $f(y)=u(a, y, \beta)$ where $u(x, y, \beta)$ is as defined in (1.3).

We will sometimes omit the third argument and simply use $u(x, y)$ to denote $u(x, y, \beta)$, if no confusion arises from the context. We use the symbol $u_{\beta}$ or $u$ to denote the measure on $[0,1] \times[0,1]$ which has density $u(x, y, \beta)$ with respect to the Lebesgue measure $\lambda$.

To prove Lemma 2.1.5, we show that any convergent subsequence of the em-

pirical measures $\left\{\frac{\pi\left(a_{n}\right)}{n}\right\}$ has limiting density $u(a, y, \beta)$ and the theorem follows from 
the standard result of convergence of measures (Theorem 2.1.12). It is unknown to us whether Lemma 2.1.5 can be obtained directly from Theorem 1.2.1. In the remainder of this section, we prove a sequence of technical lemmas to show Lemma 2.1.5. The following lemma says that the result of Theorem 1.2.1 also holds when $f$ is an indicator function of any rectangle.

Lemma 2.1.6. Under the same conditions as in Theorem 1.2.1, for any $\epsilon>0$,

$$
\lim _{n \rightarrow \infty} \mu_{n, q_{n}}\left(\left|\frac{1}{n} \sum_{i=1}^{n} \mathbb{1}_{R}\left(\frac{i}{n}, \frac{\pi(i)}{n}\right)-\int_{R} u(x, y) d x d y\right|>\epsilon\right)=0
$$

for any $R=\left[x_{1}, x_{2}\right] \times\left[y_{1}, y_{2}\right] \subset[0,1] \times[0,1]$.

Proof. First we show that for any $R=\left[x_{1}, x_{2}\right] \times\left[y_{1}, y_{2}\right]$ and any $\epsilon>0$, when $n$ is sufficiently large,

$$
L_{\pi}(R)<\min \left(x_{2}-x_{1}, y_{2}-y_{1}\right)+\frac{\epsilon}{24}
$$

for any $\pi \in S_{n}$. Let $s:=\min \left(x_{2}-x_{1}, y_{2}-y_{1}\right)$. For any $\pi \in S_{n}$, we have

$$
\left|\left\{i:\left(\frac{i}{n}, \frac{\pi(i)}{n}\right) \in R\right\}\right| \leq n s+1
$$

since, of the points in $\left\{\left(\frac{i}{n}, \frac{\pi(i)}{n}\right)\right\}$, there is one and only one point on each line $x=\frac{i}{n}$ or $y=\frac{j}{n}$. Hence, $L_{\pi}(R) \leq s+\frac{1}{n}$ for any $\pi \in S_{n}$. We can choose $n$ large enough such that $\frac{1}{n}<\frac{\epsilon}{24}$.

Next, given $\delta>0$, let $R_{\delta}:=\left(x_{1}-\delta, x_{2}+\delta\right) \times\left(y_{1}-\delta, y_{2}+\delta\right)$. Let $D:=R_{\delta}-R$. Then, it is easily seen that $D$ can be covered by four rectangles each of whose smaller side is no greater than $\delta$. For any $\delta>0$, by Urysohn's lemma (cf. 12.1 in [24]), we can choose a continuous function $f_{R_{\delta}}(x, y)$, such that,

$$
\begin{cases}f_{R_{\delta}}(x, y)=1 & \text { if }(x, y) \in R \\ f_{R_{\delta}}(x, y)=0 & \text { if }(x, y) \notin R_{\delta} \\ 0 \leq f_{R_{\delta}}(x, y) \leq 1 & \text { if }(x, y) \in D\end{cases}
$$

By the triangle inequality, we have

$$
\left|L_{\pi}(R)-u(R)\right|>\epsilon
$$




$$
\Rightarrow\left|L_{\pi}\left(f_{R_{\delta}}\right)-L_{\pi}(R)\right|+\left|u\left(f_{R_{\delta}}\right)-u(R)\right|+\left|L_{\pi}\left(f_{R_{\delta}}\right)-u\left(f_{R_{\delta}}\right)\right|>\epsilon .
$$

If we choose $\delta<\frac{\epsilon}{24}$, by (2.2), we have,

$$
\left|L_{\pi}\left(f_{R_{\delta}}\right)-L_{\pi}(R)\right| \leq L_{\pi}\left(R_{\delta}\right)-L_{\pi}(R)=L_{\pi}(D)<4\left(\frac{\epsilon}{24}+\frac{\epsilon}{24}\right)=\frac{\epsilon}{3},
$$

for any $\pi \in S_{n}$, when $n$ is sufficiently large. Since $u$ is absolutely continuous with respect to the Lebesgue measure, we may choose $\delta$ small enough such that

$$
\left|u\left(f_{R_{\delta}}\right)-u(R)\right| \leq u(D)<\frac{\epsilon}{3}
$$

Then by (2.3), for sufficiently large $n$, we have,

$$
\left|L_{\pi}(R)-u(R)\right|>\epsilon \Rightarrow\left|L_{\pi}\left(f_{R_{\delta}}\right)-u\left(f_{R_{\delta}}\right)\right|>\frac{\epsilon}{3}
$$

Thus,

$$
\mu_{n, q}\left(\left|L_{\pi}(R)-u(R)\right|>\epsilon\right) \leq \mu_{n, q}\left(\left|L_{\pi}\left(f_{R_{\delta}}\right)-u\left(f_{R_{\delta}}\right)\right|>\frac{\epsilon}{3}\right) .
$$

The lemma follows by Theorem 1.2.1.

The following property of Mallows permutations will be used in later proofs. It says that in a Mallows permutation, the relative chance that $\pi(i)$ takes two different values can be bounded in terms of the difference of those two values.

Lemma 2.1.7. For any $1 \leq i, s, t \leq n$ and $q>0$,

$$
\min \left(q^{d}, q^{-d}\right) \leq \frac{\mu_{n, q}(\pi(i)=s)}{\mu_{n, q}(\pi(i)=t)} \leq \max \left(q^{d}, q^{-d}\right),
$$

where $d=|s-t|$.

Proof. Suppose $0<q<1$. We claim it suffices to show that

$$
q \leq \frac{\mu_{n, q}(\pi(i)=j+1)}{\mu_{n, q}(\pi(i)=j)} \leq \frac{1}{q}
$$

for any $j \in[n-1]$. This follows since by taking the reciprocal of (2.4), we get

$$
q \leq \frac{\mu_{n, q}(\pi(i)=j)}{\mu_{n, q}(\pi(i)=j+1)} \leq \frac{1}{q},
$$


and the lemma follows by induction on $d$.

Consider the bijection $T_{j}$ on $S_{n}: \pi \rightarrow(j, j+1) \circ \pi$. Here $\circ$ denotes the group operator of $S_{n}$, and $(j, j+1)$ denotes the transposition of $j$ and $j+1$. Specifically, for any $i \in[n]$

$$
T_{j}(\pi)(i)= \begin{cases}j & \text { if } \pi(i)=j+1 \\ j+1 & \text { if } \pi(i)=j \\ \pi(i) & \text { otherwise. }\end{cases}
$$

From the definition, it is not hard to see that $\left|l(\pi)-l\left(T_{j}(\pi)\right)\right|=1$, for any $\pi \in S_{n}$. Hence,

$$
q \leq \frac{\mu_{n, q}\left(T_{j}(\pi)\right)}{\mu_{n, q}(\pi)} \leq \frac{1}{q}
$$

Let $A_{i, j}:=\left\{\pi \in S_{n}: \pi(i)=j\right\}$. For any fixed $i \in[n], T_{j}$ is also a bijection of $A_{i, j}$ and $A_{i, j+1}$. Hence,

$$
\frac{\mu_{n, q}(\pi(i)=j+1)}{\mu_{n, q}(\pi(i)=j)}=\frac{\sum_{\pi \in A_{i, j}} \mu_{n, q}\left(T_{j}(\pi)\right)}{\sum_{\pi \in A_{i, j}} \mu_{n, q}(\pi)},
$$

and (2.4) follows from (2.5) and (2.6). For the case $q>1$, the proof is similar. The lemma clearly also holds when $q=1$, which corresponds to the uniform measure on $S_{n}$

The following result establishes some bounds on the probability of a point in a Mallows permutation being within an interval.

Lemma 2.1.8. Suppose that $\left(q_{n}\right)_{n=1}^{\infty}$ is a sequence such that the limit $\beta=\lim _{n \rightarrow \infty} n(1-$ $\left.q_{n}\right)$ exists. For any sequence $\left\{a_{n}\right\}$ with $a_{n} \in[n]$ and any $0 \leq y_{1}<y_{2} \leq 1$,

$$
\begin{aligned}
& \limsup _{n \rightarrow \infty} \mu_{n, q_{n}}\left(\frac{\pi\left(a_{n}\right)}{n} \in\left[y_{1}, y_{2}\right]\right) \leq\left(y_{2}-y_{1}\right) e^{|\beta|}, \\
& \liminf _{n \rightarrow \infty} \mu_{n, q_{n}}\left(\frac{\pi\left(a_{n}\right)}{n} \in\left(y_{1}, y_{2}\right)\right) \geq\left(y_{2}-y_{1}\right) e^{-|\beta|} .
\end{aligned}
$$

Proof. Here we only prove the case $\beta \geq 0$. The case $\beta<0$ follows from the same argument. We also assume that $y_{2}-y_{1}<1$, since the case $y_{0}=0, y_{1}=1$ can be verified easily. Since $\lim _{n \rightarrow \infty} n\left(1-q_{n}\right)=\beta$ and $\lim _{n \rightarrow \infty} \frac{n \log q_{n}}{n\left(1-q_{n}\right)}=-1$, we have

$$
\lim _{n \rightarrow \infty} q_{n}^{n}=\lim _{n \rightarrow \infty} e^{n \log q_{n}}=e^{-\beta}
$$


Thus, for any $\delta>1$, there exists $N>0$ such that $q_{n}^{n} \in\left(\frac{e^{-\beta}}{\delta}, \delta e^{-\beta}\right)$, when $n>N$. By Lemma 2.1.7, for any $n>N$ and any $i, s, t \in[n]$

$$
\frac{\mu_{n, q_{n}}(\pi(i)=s)}{\mu_{n, q_{n}}(\pi(i)=t)} \leq \max \left(q_{n}^{n}, \frac{1}{q_{n}^{n}}\right)<\delta e^{\beta} .
$$

Let $d=y_{2}-y_{1}$ and $p_{n}=\min _{\left\{t: \frac{t}{n} \notin\left[y_{1}, y_{2}\right]\right\}}\left(\mu_{n, q_{n}}\left(\pi\left(a_{n}\right)=t\right)\right)$. Note that the set $\left\{t: \frac{t}{n} \notin\right.$ $\left.\left[y_{1}, y_{2}\right]\right\}$ is nonempty for sufficiently large $n$. Then, by (2.9) and the fact that,

$$
\left|\left\{k \in[n]: \frac{k}{n} \in\left[y_{1}, y_{2}\right]\right\}\right| \leq n d+1, \quad\left|\left\{k \in[n]: \frac{k}{n} \notin\left[y_{1}, y_{2}\right]\right\}\right| \geq n(1-d)-1
$$

we have,

$$
\begin{aligned}
& \mu_{n, q_{n}}\left(\frac{\pi\left(a_{n}\right)}{n} \in\left[y_{1}, y_{2}\right]\right)<(n d+1) \delta e^{\beta} p_{n}, \\
& \mu_{n, q_{n}}\left(\frac{\pi\left(a_{n}\right)}{n} \notin\left[y_{1}, y_{2}\right]\right) \geq(n(1-d)-1) p_{n} .
\end{aligned}
$$

Hence,

$$
\begin{aligned}
\mu_{n, q_{n}}\left(\frac{\pi\left(a_{n}\right)}{n} \in\left[y_{1}, y_{2}\right]\right) & <\frac{(n d+1) \delta e^{\beta}}{(n(1-d)-1)+(n d+1) \delta e^{\beta}} \\
& <\frac{(n d+1) \delta e^{\beta}}{(n(1-d)-1)+(n d+1)} \\
& =\frac{(n d+1) \delta e^{\beta}}{n}
\end{aligned}
$$

and (2.7) follows since $\delta$ can be chosen arbitrarily close to 1. Similarly, to prove (2.8), define $p_{n}^{\prime}=\min _{\left\{t: \frac{t}{n} \in\left(y_{1}, y_{2}\right)\right\}}\left(\mu_{n, q_{n}}\left(\pi\left(a_{n}\right)=t\right)\right)$. Then, by (2.9) and the fact that,

$$
\left|\left\{k \in[n]: \frac{k}{n} \in\left(y_{1}, y_{2}\right)\right\}\right| \geq n d-1, \quad\left|\left\{k \in[n]: \frac{k}{n} \notin\left(y_{1}, y_{2}\right)\right\}\right| \leq n(1-d)+1,
$$

we have

$$
\begin{aligned}
& \mu_{n, q_{n}}\left(\frac{\pi\left(a_{n}\right)}{n} \in\left(y_{1}, y_{2}\right)\right) \geq(n d-1) p_{n}^{\prime} \\
& \mu_{n, q_{n}}\left(\frac{\pi\left(a_{n}\right)}{n} \notin\left(y_{1}, y_{2}\right)\right)<(n(1-d)+1) \delta e^{\beta} p_{n}^{\prime}
\end{aligned}
$$

Hence,

$$
\mu_{n, q_{n}}\left(\frac{\pi\left(a_{n}\right)}{n} \in\left(y_{1}, y_{2}\right)\right)>\frac{(n d-1)}{(n(1-d)+1) \delta e^{\beta}+(n d-1)}
$$




$$
\begin{aligned}
& >\frac{(n d-1)}{(n(1-d)+1) \delta e^{\beta}+(n d-1) \delta e^{\beta}} \\
& =\frac{(n d-1) e^{-\beta}}{n \delta} .
\end{aligned}
$$

(2.8) follows since $\delta$ can be chosen arbitrarily close to 1 .

In the next two lemmas, we introduce some properties of the density function $u(x, y, \beta)$ defined in Theorem 1.2.1.

Lemma 2.1.9. With $u(x, y, \beta)$ defined as in (1.3), we have

$$
\begin{aligned}
& \int_{0}^{1} u(x, y, \beta) d x=1, \quad \forall y \in[0,1], \\
& \int_{0}^{1} u(x, y, \beta) d y=1, \quad \forall x \in[0,1] .
\end{aligned}
$$

Proof. Since $\cosh (x)$ is an even function, $u(x, y, \beta)$ is symmetric with respect to the line $y=x$. That is

$$
u(x, y, \beta)=u(y, x, \beta), \quad \forall x, y \in[0,1] .
$$

Hence we only need to show the first identity. By Corollary 6.2 in [26],

$$
\frac{\partial^{2} \ln u(x, y, \beta)}{\partial x \partial y}=2 \beta u(x, y, \beta) .
$$

Therefore, we have

$$
\int_{0}^{1} u(x, y, \beta) d x=\frac{1}{2 \beta}\left(\frac{\partial \ln u(1, y, \beta)}{\partial y}-\frac{\partial \ln u(0, y, \beta)}{\partial y}\right) .
$$

Next, by direct calculation, we have

$$
\begin{aligned}
& u(1, y, \beta)=\frac{(\beta / 2) \sinh (\beta / 2)}{\left(\frac{1}{2} e^{-\frac{\beta}{4}}\left(e^{\beta}-1\right) e^{-\frac{\beta y}{2}}\right)^{2}}=\frac{\beta e^{\beta y}}{e^{\beta}-1}, \\
& u(0, y, \beta)=\frac{(\beta / 2) \sinh (\beta / 2)}{\left(\frac{1}{2} e^{\frac{\beta}{4}}\left(1-e^{-\beta}\right) e^{\frac{\beta y}{2}}\right)^{2}}=\frac{\beta e^{-\beta y}}{1-e^{-\beta}} .
\end{aligned}
$$

Hence, we get

$$
\frac{\partial \ln u(1, y, \beta)}{\partial y}=\beta \quad \text { and } \quad \frac{\partial \ln u(0, y, \beta)}{\partial y}=-\beta .
$$

By (2.11), the lemma follows. 
In the remainder of this section, we will simply use $u(x, y)$ to denote $u(x, y, \beta)$.

Lemma 2.1.10. For any $0 \leq a, c, d \leq 1$,

$$
-\beta \int_{c}^{d}\left(-\int_{0}^{a} u(x, y) d x+\int_{a}^{1} u(x, y) d x\right) d y=\ln \frac{u(a, d)}{u(a, c)} .
$$

Proof. For fixed $c, d \in[0,1]$, define $f(a)$ to be the left-hand side of the identity and $g(a)$ to be the right-hand side of the identity. Then, by Lemma 2.1.9 and (2.13), we have

$$
f(0)=g(0)=\beta(c-d)
$$

Hence, to prove the identity it suffices to show $f^{\prime}(a)=g^{\prime}(a)$ for any $a \in(0,1)$. Since $u(x, y)$ is bounded on $[0,1] \times[0,1]$, we can change the order of integral and differentiation in the following,

$$
\begin{aligned}
f^{\prime}(a) & =-\beta \frac{\partial}{\partial a} \int_{c}^{d}\left(-\int_{0}^{a} u(x, y) d x+\int_{a}^{1} u(x, y) d x\right) d y \\
& =-\beta \int_{c}^{d} \frac{\partial}{\partial a}\left(-\int_{0}^{a} u(x, y) d x+\int_{a}^{1} u(x, y) d x\right) d y \\
& =-\beta \int_{c}^{d}(-u(a, y)-u(a, y)) d y \\
& =2 \beta \int_{c}^{d} u(a, y) d y .
\end{aligned}
$$

By $(2.10), \frac{\partial \ln u(x, y)}{\partial x}$ is the anti-derivative of $2 \beta u(x, y)$ with respect to $y$. Thus we have

$$
g^{\prime}(a)=\frac{\partial \ln u(a, d)}{\partial a}-\frac{\partial \ln u(a, c)}{\partial a}=2 \beta \int_{c}^{d} u(a, y) d y
$$

Lemma 2.1.11. In the context of Lemma 2.1.8, suppose $\left\{a_{n}\right\}_{n \geq 1}$ is a sequence such that $\lim _{n \rightarrow \infty} \frac{a_{n}}{n}=a$, where $a_{n} \in[n]$. For any $0 \leq y_{1}<y_{2}<1$,

$$
\lim _{\epsilon \rightarrow 0^{+}} \limsup _{n \rightarrow \infty}\left|\frac{\mu_{n, q_{n}}\left(\frac{\pi\left(a_{n}\right)}{n} \in\left(y_{2}, y_{2}+\epsilon\right)\right)}{\mu_{n, q_{n}}\left(\frac{\pi\left(a_{n}\right)}{n} \in\left(y_{1}, y_{1}+\epsilon\right)\right)}-\frac{u\left(a, y_{2}\right)}{u\left(a, y_{1}\right)}\right|=0 \text {. }
$$

For any $0<y_{1}<y_{2} \leq 1$,

$$
\lim _{\epsilon \rightarrow 0^{+}} \limsup _{n \rightarrow \infty}\left|\frac{\mu_{n, q_{n}}\left(\frac{\pi\left(a_{n}\right)}{n} \in\left(y_{2}-\epsilon, y_{2}\right)\right)}{\mu_{n, q_{n}}\left(\frac{\pi\left(a_{n}\right)}{n} \in\left(y_{1}-\epsilon, y_{1}\right)\right)}-\frac{u\left(a, y_{2}\right)}{u\left(a, y_{1}\right)}\right|=0 \text {. }
$$


Proof. Here we only prove (2.14), since (2.15) follows from the similar argument.

To prove (2.14), we need to show that for any $\eta>0$, there exists $\epsilon_{0}>0$ such that for any fixed $\epsilon<\epsilon_{0}$, there exists $N>0$, which may depend on $\epsilon$, such that for any $n>N$, we have

$$
\left|\frac{\mu_{n, q_{n}}\left(\frac{\pi\left(a_{n}\right)}{n} \in\left(y_{2}, y_{2}+\epsilon\right)\right)}{\mu_{n, q_{n}}\left(\frac{\pi\left(a_{n}\right)}{n} \in\left(y_{1}, y_{1}+\epsilon\right)\right)}-\frac{u\left(a, y_{2}\right)}{u\left(a, y_{1}\right)}\right|<\eta .
$$

First, we define the following two rectangles:

$$
R_{0}:=[0, a] \times\left[y_{1}, y_{2}\right], \quad R_{1}:=[a, 1] \times\left[y_{1}, y_{2}\right]
$$

Next define

$$
G(n, \lambda):=\left\{\pi \in S_{n}:\left|L_{\pi}(A)-u(A)\right|<\lambda, \text { for any } A \in\left\{R_{0}, R_{1}\right\}\right\}
$$

Let $\bar{G}(n, \lambda):=S_{n} \backslash G(n, \lambda)$ denote the complement of $G(n, \lambda)$. Then,

$$
\bar{G}(n, \lambda)=\cup_{A \in\left\{R_{0}, R_{1}\right\}}\left\{\pi \in S_{n}:\left|L_{\pi}(A)-u(A)\right| \geq \lambda\right\} .
$$

Thus by Lemma 2.1.6, for any $\epsilon_{0}>0$ and any $\lambda>0$, we have

$$
\lim _{n \rightarrow \infty} \mu_{n, q_{n}}(\bar{G}(n, \lambda))=0
$$

Define

$$
G D(n, \lambda):=\left\{\pi \in S_{n}: Q\left(\pi, a_{n}\right) \cap G(n, \lambda / 2) \neq \varnothing\right\}
$$

Note that, for any rectangle $R$ and any $\tau, \xi \in Q\left(\pi, a_{n}\right)$,

$$
\left|L_{\tau}(R)-L_{\xi}(R)\right| \leq \frac{1}{n}
$$

Thus, when $n>\frac{2}{\lambda}$, it follows from triangle inequality that

$$
G D(n, \lambda) \subset G(n, \lambda)
$$

On the other hand, by the definition of $G D(n, \lambda)$ and the fact that, for any $i \in[n]$, $\pi \in Q(\pi, i)$, it follows that

$$
G(n, \lambda / 2) \subset G D(n, \lambda)
$$


Hence by (2.17) and (2.19), for any $\lambda>0$, we have

$$
\lim _{n \rightarrow \infty} \mu_{n, q_{n}}(G D(n, \lambda))=1
$$

Next, given $\epsilon \in\left(0, \epsilon_{0}\right)$ where the value of $\epsilon_{0}$ is to be determined, define

$$
A_{n}:=\left\{\pi \in S_{n}: \frac{\pi\left(a_{n}\right)}{n} \in\left(y_{1}, y_{1}+\epsilon\right)\right\}, \quad B_{n}:=\left\{\pi \in S_{n}: \frac{\pi\left(a_{n}\right)}{n} \in\left(y_{2}, y_{2}+\epsilon\right)\right\}
$$

Then, by Lemma 2.1.8, when $n$ is sufficiently large, we have

$$
\mu_{n, q_{n}}\left(A_{n}\right)>\frac{\epsilon}{2} e^{-|\beta|}, \quad \mu_{n, q_{n}}\left(B_{n}\right)>\frac{\epsilon}{2} e^{-|\beta|} .
$$

Thus, by (2.20), there exists an $N_{1}>0$ such that, for any $n>N_{1}$, we have

$$
\left|\frac{\mu_{n, q_{n}}\left(B_{n} \cap G D(n, \lambda)\right)}{\mu_{n, q_{n}}\left(A_{n} \cap G D(n, \lambda)\right)}-\frac{\mu_{n, q_{n}}\left(B_{n}\right)}{\mu_{n, q_{n}}\left(A_{n}\right)}\right|<\frac{\eta}{2} .
$$

Therefore, to prove (2.16), it suffices to show that for sufficiently large $n$, we have

$$
\left|\frac{\mu_{n, q_{n}}\left(B_{n} \cap G D(n, \lambda)\right)}{\mu_{n, q_{n}}\left(A_{n} \cap G D(n, \lambda)\right)}-\frac{u\left(a, y_{2}\right)}{u\left(a, y_{1}\right)}\right|<\frac{\eta}{2} .
$$

In order to prove $(2.21)$, we are going to exploit two things. The first one is the fact that $\left\{Q\left(\pi, a_{n}\right): \pi \in G D(n, \lambda)\right\}$ is a partition of $G D(n, \lambda)$. The second is the following,

$$
\begin{aligned}
& \frac{c_{i}}{d_{i}}>r, c_{i}>0, d_{i}>0 \text { for } \forall i \in[m] \Rightarrow \frac{\sum_{i=1}^{m} c_{i}}{\sum_{i=1}^{m} d_{i}}>r, \\
& \frac{c_{i}}{d_{i}}<r, c_{i}>0, d_{i}>0 \text { for } \forall i \in[m] \Rightarrow \frac{\sum_{i=1}^{m} c_{i}}{\sum_{i=1}^{m} d_{i}}<r .
\end{aligned}
$$

Hence, to prove (2.21), it suffices to show that, for sufficiently large $n$, we have

$$
\left|\frac{\mu_{n, q_{n}}\left(B_{n} \cap Q\left(\pi, a_{n}\right)\right)}{\mu_{n, q_{n}}\left(A_{n} \cap Q\left(\pi, a_{n}\right)\right)}-\frac{u\left(a, y_{2}\right)}{u\left(a, y_{1}\right)}\right|<\frac{\eta}{2},
$$

for any $Q\left(\pi, a_{n}\right) \subset G D(n, \lambda)$. Note that $A_{n} \cap Q\left(\pi, a_{n}\right)$ is nonempty for any $\pi \in S_{n}$, when $n>1 / \epsilon$. The strategy to prove (2.22) is the following, we show that when $n$ is sufficiently large, for any $Q\left(\pi, a_{n}\right) \subset G D(n, \lambda)$ and any $\tau \in B_{n} \cap Q\left(\pi, a_{n}\right), \xi \in$ $A_{n} \cap Q\left(\pi, a_{n}\right)$, we have

$$
\left|\frac{1}{n}(l(\tau)-l(\xi))-I\right|<2 \lambda+4 \epsilon+\frac{4}{n} .
$$


Here

$$
I:=\int_{y_{1}}^{y_{2}}\left(-\int_{0}^{a} u(x, y) d x+\int_{a}^{1} u(x, y) d x\right) d y=u\left(R_{1}\right)-u\left(R_{0}\right) .
$$

Note that $\frac{\mu_{n, q_{n}}(\tau)}{\mu_{n, q_{n}}(\xi)}=q_{n}^{l(\tau)-l(\xi)}$. Thus, by (2.23), for any $\tau \in B_{n} \cap Q\left(\pi, a_{n}\right), \xi \in A_{n} \cap$ $Q\left(\pi, a_{n}\right)$, we have

$$
q_{n}^{n(I+2 \lambda+4 \epsilon+4 / n)} \leq \frac{\mu_{n, q_{n}}(\tau)}{\mu_{n, q_{n}}(\xi)} \leq q_{n}^{n(I-2 \lambda-4 \epsilon-4 / n)}
$$

Here we assume $0<q_{n}<1$. (The cases $q_{n}>1$ and $q_{n}=1$ follow by similar argument.) By the definition of $A_{n}, B_{n}$, we have

$$
n \epsilon-1 \leq\left|A_{n} \cap Q\left(\pi, a_{n}\right)\right|,\left|B_{n} \cap Q\left(\pi, a_{n}\right)\right| \leq n \epsilon+1 .
$$

Hence we have

$$
\frac{n \epsilon-1}{n \epsilon+1} q_{n}^{n(I+2 \lambda+4 \epsilon+4 / n)} \leq \frac{\mu_{n, q_{n}}\left(B_{n} \cap Q\left(\pi, a_{n}\right)\right)}{\mu_{n, q_{n}}\left(A_{n} \cap Q\left(\pi, a_{n}\right)\right)} \leq \frac{n \epsilon+1}{n \epsilon-1} q_{n}^{n(I-2 \lambda-4 \epsilon-4 / n)} .
$$

By Lemma 2.1.10 and the fact that $\lim _{n \rightarrow \infty} q_{n}^{n}=e^{-\beta}$ and $\lim _{n \rightarrow \infty} q_{n}=1$, we have

$$
\begin{aligned}
& \lim _{n \rightarrow \infty} \frac{n \epsilon-1}{n \epsilon+1} q_{n}^{n(I+2 \lambda+4 \epsilon+4 / n)}=\frac{u\left(a, y_{2}\right)}{u\left(a, y_{1}\right)} e^{-\beta(2 \lambda+4 \epsilon)}, \\
& \lim _{n \rightarrow \infty} \frac{n \epsilon+1}{n \epsilon-1} q_{n}^{n(I-2 \lambda-4 \epsilon-4 / n)}=\frac{u\left(a, y_{2}\right)}{u\left(a, y_{1}\right)} e^{\beta(2 \lambda+4 \epsilon)} .
\end{aligned}
$$

Thus, we can choose $\epsilon_{0}$ and $\lambda$ small enough such that, for any $\epsilon \in\left(0, \epsilon_{0}\right),(2.22)$ holds for sufficiently large $n$.

The remaining part of the proof is to show (2.23). Suppose $n$ is sufficiently large such that $\frac{a_{n}}{n} \in(a-\epsilon, a+\epsilon)$. Without loss of generality, suppose $\frac{a_{n}}{n} \in[a, a+\epsilon)$. (The other case can be shown in a similar argument.) By Proposition 2.1.4, for any $Q\left(\pi, a_{n}\right) \subset G D(n, \lambda)$, and for any $\tau \in B_{n} \cap Q\left(\pi, a_{n}\right), \xi \in A_{n} \cap Q\left(\pi, a_{n}\right)$, we have

$$
\begin{aligned}
& l(\tau)-l(\xi) \\
= & \left|\left\{t>a_{n}: \xi\left(a_{n}\right)<\xi(t) \leq \tau\left(a_{n}\right)\right\}\right|-\left|\left\{t<a_{n}: \xi\left(a_{n}\right)<\xi(t) \leq \tau\left(a_{n}\right)\right\}\right| \\
= & \left|\left\{\frac{t}{n}>\frac{a_{n}}{n}: \frac{\xi\left(a_{n}\right)}{n}<\frac{\xi(t)}{n} \leq \frac{\tau\left(a_{n}\right)}{n}\right\}\right|-\left|\left\{\frac{t}{n}<\frac{a_{n}}{n}: \frac{\xi\left(a_{n}\right)}{n}<\frac{\xi(t)}{n} \leq \frac{\tau\left(a_{n}\right)}{n}\right\}\right|
\end{aligned}
$$




$$
\begin{aligned}
& \leq\left|\left\{\frac{t}{n}>a: y_{1}<\frac{\xi(t)}{n}<y_{2}+\epsilon\right\}\right|-\left|\left\{\frac{t}{n}<a: y_{1}+\epsilon \leq \frac{\xi(t)}{n} \leq y_{2}\right\}\right| \\
& =\left|\left\{t:\left(\frac{t}{n}, \frac{\xi(t)}{n}\right) \in(a, 1] \times\left(y_{1}, y_{2}+\epsilon\right)\right\}\right| \\
& -\left|\left\{t:\left(\frac{t}{n}, \frac{\xi(t)}{n}\right) \in(0, a) \times\left[y_{1}+\epsilon, y_{2}\right]\right\}\right| \\
& \leq\left|\left\{t:\left(\frac{t}{n}, \frac{\xi(t)}{n}\right) \in(a, 1] \times\left(y_{1}, y_{2}\right]\right\}\right|+(n \epsilon+1) \\
& -\left|\left\{t:\left(\frac{t}{n}, \frac{\xi(t)}{n}\right) \in(0, a) \times\left[y_{1}, y_{2}\right]\right\}\right|+(n \epsilon+1) \\
& \leq\left|\left\{t:\left(\frac{t}{n}, \frac{\xi(t)}{n}\right) \in[a, 1] \times\left[y_{1}, y_{2}\right]\right\}\right| \\
& -\left|\left\{t:\left(\frac{t}{n}, \frac{\xi(t)}{n}\right) \in[0, a] \times\left[y_{1}, y_{2}\right]\right\}\right|+2 n \epsilon+4 \\
& =n L_{\xi}\left([a, 1] \times\left[y_{1}, y_{2}\right]\right)-n L_{\xi}\left([0, a] \times\left[y_{1}, y_{2}\right]\right)+2 n \epsilon+4 \\
& =n L_{\xi}\left(R_{1}\right)-n L_{\xi}\left(R_{0}\right)+2 n \epsilon+4 \text {. }
\end{aligned}
$$

The first inequality above follows because $\frac{a_{n}}{n} \geq a, \frac{\xi\left(a_{n}\right)}{n} \in\left(y_{1}, y_{1}+\epsilon\right)$ and $\frac{\tau\left(a_{n}\right)}{n} \in\left(y_{2}, y_{2}+\epsilon\right)$. The second inequality follows because

$$
\begin{aligned}
& \left|\left\{t \in[n]: \frac{\xi(t)}{n} \in\left(y_{2}, y_{2}+\epsilon\right)\right\}\right| \leq n \epsilon+1, \\
& \left|\left\{t \in[n]: \frac{\xi(t)}{n} \in\left[y_{1}, y_{1}+\epsilon\right)\right\}\right| \leq n \epsilon+1 .
\end{aligned}
$$

The third inequality follows because, since we change $(0, a)$ to $[0, a]$ in the second term, we add two in the end to compensate the possible extra subtraction. Hence, we have

$$
\begin{aligned}
\frac{1}{n}(l(\tau)-l(\xi)) & \leq L_{\xi}\left(R_{1}\right)-L_{\xi}\left(R_{0}\right)+2 \epsilon+\frac{4}{n} \\
& \leq u\left(R_{1}\right)-u\left(R_{0}\right)+2 \lambda+2 \epsilon+\frac{4}{n} \\
& =I+2 \lambda+2 \epsilon+\frac{4}{n} .
\end{aligned}
$$

Here we use the fact that, by (2.18), $\xi \in G D(n, \lambda) \subset G(n, \lambda)$.

Similarly, to show the lower bound of $l(\tau)-l(\xi)$, we have

$$
\begin{aligned}
& l(\tau)-l(\xi) \\
= & \left|\left\{t>a_{n}: \xi\left(a_{n}\right)<\xi(t) \leq \tau\left(a_{n}\right)\right\}\right|-\left|\left\{t<a_{n}: \xi\left(a_{n}\right)<\xi(t) \leq \tau\left(a_{n}\right)\right\}\right| \\
= & \left|\left\{\frac{t}{n}>\frac{a_{n}}{n}: \frac{\xi\left(a_{n}\right)}{n}<\frac{\xi(t)}{n} \leq \frac{\tau\left(a_{n}\right)}{n}\right\}\right|-\left|\left\{\frac{t}{n}<\frac{a_{n}}{n}: \frac{\xi\left(a_{n}\right)}{n}<\frac{\xi(t)}{n} \leq \frac{\tau\left(a_{n}\right)}{n}\right\}\right| \\
= & \left|\left\{\frac{t}{n}>a: \frac{\xi\left(a_{n}\right)}{n}<\frac{\xi(t)}{n} \leq \frac{\tau\left(a_{n}\right)}{n}\right\}\right|-\left|\left\{\frac{a_{n}}{n} \geq \frac{t}{n}>a: \frac{\xi\left(a_{n}\right)}{n}<\frac{\xi(t)}{n} \leq \frac{\tau\left(a_{n}\right)}{n}\right\}\right|
\end{aligned}
$$




$$
\begin{aligned}
& -\left|\left\{\frac{t}{n}<a: \frac{\xi\left(a_{n}\right)}{n}<\frac{\xi(t)}{n} \leq \frac{\tau\left(a_{n}\right)}{n}\right\}\right|-\left|\left\{a \leq \frac{t}{n}<\frac{a_{n}}{n}: \frac{\xi\left(a_{n}\right)}{n}<\frac{\xi(t)}{n} \leq \frac{\tau\left(a_{n}\right)}{n}\right\}\right| \\
& \geq\left|\left\{\frac{t}{n}>a: y_{1}+\epsilon \leq \frac{\xi(t)}{n} \leq y_{2}\right\}\right|-(n \epsilon+1) \\
& -\left|\left\{\frac{t}{n}<a: y_{1}<\frac{\xi(t)}{n}<y_{2}+\epsilon\right\}\right|-(n \epsilon+1) \\
& =\left|\left\{t:\left(\frac{t}{n}, \frac{\xi(t)}{n}\right) \in(a, 1] \times\left[y_{1}+\epsilon, y_{2}\right]\right\}\right| \\
& -\left|\left\{t:\left(\frac{t}{n}, \frac{\xi(t)}{n}\right) \in(0, a) \times\left(y_{1}, y_{2}+\epsilon\right)\right\}\right|-2(n \epsilon+1) \\
& \geq\left|\left\{t:\left(\frac{t}{n}, \frac{\xi(t)}{n}\right) \in(a, 1] \times\left[y_{1}, y_{2}\right]\right\}\right|-(n \epsilon+1) \\
& -\left|\left\{t:\left(\frac{t}{n}, \frac{\xi(t)}{n}\right) \in(0, a) \times\left(y_{1}, y_{2}\right]\right\}\right|-(n \epsilon+1)-2(n \epsilon+1) \\
& =n L_{\xi}\left([a, 1] \times\left[y_{1}, y_{2}\right]\right)-n L_{\xi}\left([0, a] \times\left[y_{1}, y_{2}\right]\right)-4 n \epsilon-4 \\
& =n L_{\xi}\left(R_{1}\right)-n L_{\xi}\left(R_{0}\right)-4 n \epsilon-4 \text {. }
\end{aligned}
$$

The first inequality above follows since, by the definition of $A_{n}, B_{n}$, we have $\frac{\xi\left(a_{n}\right)}{n} \in$ $\left(y_{1}, y_{1}+\epsilon\right), \frac{\tau\left(a_{n}\right)}{n} \in\left(y_{2}, y_{2}+\epsilon\right)$ and, since $\frac{a_{n}}{n} \in[a, a+\epsilon)$,

$$
\left|\left\{t \in[n]: \frac{a_{n}}{n} \geq \frac{t}{n}>a\right\}\right| \leq n \epsilon+1, \quad\left|\left\{t \in[n]: a \leq \frac{t}{n}<\frac{a_{n}}{n}\right\}\right| \leq n \epsilon+1 .
$$

The second inequality follows because

$$
\begin{aligned}
& \left|\left\{t \in[n]: \frac{\xi(t)}{n} \in\left[y_{1}, y_{1}+\epsilon\right)\right\}\right| \leq n \epsilon+1, \\
& \left|\left\{t \in[n]: \frac{\xi(t)}{n} \in\left(y_{2}, y_{2}+\epsilon\right)\right\}\right| \leq n \epsilon+1 .
\end{aligned}
$$

Hence, we have

$$
\begin{aligned}
\frac{1}{n}(l(\tau)-l(\xi)) & \geq L_{\xi}\left(R_{1}\right)-L_{\xi}\left(R_{0}\right)-4 \epsilon-\frac{4}{n} \\
& \geq u\left(R_{1}\right)-u\left(R_{0}\right)-2 \lambda-4 \epsilon-\frac{4}{n} \\
& =I-2 \lambda-4 \epsilon-\frac{4}{n} .
\end{aligned}
$$

Here again we use the fact that, by (2.18), $\xi \in G D(n, \lambda) \subset G(n, \lambda)$. The fact that (2.23) follows from (2.24) and (2.25) completes the proof.

To complete the proof of Lemma 2.1.5 we use the following result (cf. 7.2.5 in [3]) and the next two lemmas. 
Theorem 2.1.12. Let $\left\{u_{n}\right\}_{n \geq 1}$ be a sequence of finite measures on $\mathbb{R}$. If $\left\{u_{n}\right\}_{n \geq 1}$ is tight, and every weakly convergent subsequence of $\left\{u_{n}\right\}_{n \geq 1}$ converges to the measure $v$, then $u_{n} \stackrel{d}{\longrightarrow} v$.

Lemma 2.1.13. In the context of Lemma 2.1.5, let $\left\{a_{t_{n}}\right\}$ be a subsequence of $\left\{a_{n}\right\}$ such that

$$
\mu_{t_{n}, q_{n}}\left(\frac{\pi\left(a_{t_{n}}\right)}{t_{n}} \in(\cdot)\right) \stackrel{d}{\longrightarrow} v .
$$

Then the distribution function $F_{v}(y)$ of the limit probability measure $v$ is absolutely continuous. Here $\mu_{t_{n}, q_{t_{n}}}\left(\frac{\pi\left(a_{t_{n}}\right)}{t_{n}} \in(\cdot)\right)$ denotes the probability measure induced by $\frac{\pi\left(a_{t_{n}}\right)}{t_{n}}$ under $\mu_{t_{n}, q_{t_{n}}}$.

Proof. For any $\epsilon>0$, let $\delta=\frac{\epsilon}{4 e^{|\beta|}}$. By the definition of absolute continuity, we will show that, for any $\left\{\left(y_{1}, y_{2}\right),\left(y_{3}, y_{4}\right), \ldots,\left(y_{2 m-1}, y_{2 m}\right)\right\}$ with $y_{2 k-1}<y_{2 k}$ and $\sum_{k=1}^{m} \mid y_{2 k}-$ $y_{2 k-1} \mid<\delta$, we have $\sum_{k=1}^{m}\left|F_{v}\left(y_{2 k}\right)-F_{v}\left(y_{2 k-1}\right)\right|<\epsilon$. Without loss of generality, we may assume that every $y_{i}$ is a continuous point of $F_{v}(y)$ with $0 \leq y_{i} \leq 1$. Since there are at most countably many discontinuity of $F_{v}(y)$, we can always choose a new set of interval $\left\{\left(y_{2 k-1}^{\prime}, y_{2 k}^{\prime}\right)\right\}$ such that $F_{v}(y)$ is continuous at every $y_{i}^{\prime},\left[y_{2 k-1}, y_{2 k}\right] \subset\left[y_{2 k-1}^{\prime}, y_{2 k}^{\prime}\right]$ and $\sum_{k=1}^{m}\left|y_{2 k}^{\prime}-y_{2 k-1}^{\prime}\right|<\delta$ still holds. Next, for the simplicity of notation, define

$$
v_{n}:=\mu_{t_{n}, q_{t_{n}}}\left(\frac{\pi\left(a_{t_{n}}\right)}{t_{n}} \in(\cdot)\right) .
$$

By Lemma 2.1.8, there exists $N_{1}>0$ such that for any $n>N_{1}$,

$$
v_{n}\left(\left[y_{2 k-1}, y_{2 k}\right]\right) \leq 2\left(y_{2 k}-y_{2 k-1}\right) e^{|\beta|},
$$

for all $k \in[m]$. Since $v_{n} \stackrel{d}{\longrightarrow} v$, there exists $N_{2}>0$ such that for any $n>N_{2}$,

$$
\left|F_{v}\left(y_{2 k}\right)-F_{v}\left(y_{2 k-1}\right)-v_{n}\left(\left[y_{2 k-1}, y_{2 k}\right]\right)\right|<\frac{\epsilon}{2 m}
$$

for all $k \in[m]$. Let $n=\max \left(N_{1}, N_{2}\right)+1$, we have

$$
\sum_{k=1}^{m}\left|F_{v}\left(y_{2 k}\right)-F_{v}\left(y_{2 k-1}\right)\right|
$$




$$
\begin{aligned}
& \leq \sum_{k=1}^{m}\left|F_{v}\left(y_{2 k}\right)-F_{v}\left(y_{2 k-1}\right)-v_{n}\left(\left[y_{2 k-1}, y_{2 k}\right]\right)\right|+\sum_{k=1}^{m} v_{n}\left(\left[y_{2 k-1}, y_{2 k}\right]\right) \\
& <\frac{\epsilon}{2}+2 e^{|\beta|} \sum_{k=1}^{m}\left(y_{2 k}-y_{2 k-1}\right) \\
& <\frac{\epsilon}{2}+2 e^{|\beta|} \delta \\
& =\epsilon
\end{aligned}
$$

Lemma 2.1.14. In the context of Lemma 2.1.13, we have

$$
F_{v}(y)=\int_{0}^{y} u(a, t, \beta) d t
$$

for any $y \in[0,1]$. Here $u(x, y, \beta)$ is defined in (1.3).

Proof. For the simplicity of notation, we will use $u(x, y)$ to denote $u(x, y, \beta)$. By Lemma 2.1.13, $F_{v}(y)$ is absolutely continuous. Hence $F_{v}(y)$ is differentiable almost everywhere, say $F_{v}^{\prime}(y)=f(y)$ a.e. on $[0,1]$, and moreover, we have $F_{v}(y)=\int_{0}^{y} f(t) d t$. Here we use the fact that the support of $v$ is $[0,1]$. Note that, by Lemma 2.1.8, for any $y \in(0,1)$ such that $F_{v}^{\prime}(y)=f(y)$, we have $f(y) \geq e^{-|\beta|}>0$. Then in order to show $f(y)=u(a, y)$ a.e., it suffices to show

$$
\frac{f\left(y_{2}\right)}{f\left(y_{1}\right)}=\frac{u\left(a, y_{2}\right)}{u\left(a, y_{1}\right)}
$$

for any $y_{1}, y_{2} \in A$, where $A:=\left\{y \in(0,1): F_{v}^{\prime}(y)=f(y)\right\}$. This is because, for any $y \in A$, we have

$$
\frac{1}{f(y)}=\int_{0}^{1} \frac{f(z)}{f(y)} d z=\int_{A} \frac{f(z)}{f(y)} d z=\int_{A} \frac{u(a, z)}{u(a, y)} d z=\int_{0}^{1} \frac{u(a, z)}{u(a, y)} d z=\frac{1}{u(a, y)} .
$$

Here we use the fact that the Lebesgue measure of $A$ is 1 as well as Lemma 2.1.9 in the last equality. Next, since we have

$$
\begin{aligned}
\lim _{\epsilon \rightarrow 0^{+}} \frac{v\left(\left(y_{2}, y_{2}+\epsilon\right)\right)}{v\left(\left(y_{1}, y_{1}+\epsilon\right)\right)} & =\lim _{\epsilon \rightarrow 0^{+}} \frac{F_{v}\left(y_{2}+\epsilon\right)-F_{v}\left(y_{2}\right)}{F_{v}\left(y_{1}+\epsilon\right)-F_{v}\left(y_{1}\right)} \\
& =\lim _{\epsilon \rightarrow 0^{+}} \frac{F_{v}\left(y_{2}+\epsilon\right)-F_{v}\left(y_{2}\right)}{\epsilon} / \frac{F_{v}\left(y_{1}+\epsilon\right)-F_{v}\left(y_{1}\right)}{\epsilon}
\end{aligned}
$$




$$
=\frac{f\left(y_{2}\right)}{f\left(y_{1}\right)} \text {. }
$$

Thus, to prove (2.27), it suffices to show that

$$
\lim _{\epsilon \rightarrow 0^{+}}\left|\frac{v\left(\left(y_{2}, y_{2}+\epsilon\right)\right)}{v\left(\left(y_{1}, y_{1}+\epsilon\right)\right)}-\frac{u\left(a, y_{2}\right)}{u\left(a, y_{1}\right)}\right|=0
$$

Next, inheriting the notation in (2.26), since $v_{n} \stackrel{d}{\longrightarrow} v$ and $F_{v}(y)$ is continuous, we have

$$
\lim _{n \rightarrow \infty}\left|\frac{v_{n}\left(\left(y_{2}, y_{2}+\epsilon\right)\right)}{v_{n}\left(\left(y_{1}, y_{1}+\epsilon\right)\right)}-\frac{u\left(a, y_{2}\right)}{u\left(a, y_{1}\right)}\right|=\left|\frac{v\left(\left(y_{2}, y_{2}+\epsilon\right)\right)}{v\left(\left(y_{1}, y_{1}+\epsilon\right)\right)}-\frac{u\left(a, y_{2}\right)}{u\left(a, y_{1}\right)}\right| .
$$

Since $\left\{v_{n}\right\}$ is a subsequence of $\left\{\mu_{n, q_{n}}\left(\frac{\pi\left(a_{n}\right)}{n} \in(\cdot)\right)\right\}$, by Lemma 2.1.11, (2.28) follows from (2.29).

Proof of Lemma 2.1.5. Since the support of $\mu_{n, q_{n}}\left(\frac{\pi\left(a_{n}\right)}{n} \in(\cdot)\right)$ is within $[0,1]$, the sequence $\left\{\mu_{n, q_{n}}\left(\frac{\pi\left(a_{n}\right)}{n} \in(\cdot)\right)\right\}$ is tight. The claim follows from Lemma 2.1.13, Lemma 2.1.14 and Theorem 2.1.12.

\subsubsection{Proof of Lemma 2.1.1 and Lemma 2.1.2}

Definition 2.1.15. For any $\pi \in S_{n}$ and any $1 \leq j<k \leq n$, let $\pi([j, k])$ denote the vector $(\pi(j), \pi(j+1), \cdots, \pi(k))$. Let $\pi_{[j, k]}$ denote the permutation in $S_{k-j+1}$ induced by $\pi([j, k])$, i.e.

$$
\pi_{[j, k]}(i):=\sum_{s=j}^{k} \mathbb{1}_{\{\pi(s) \leq \pi(j+i-1)\}}, \quad \forall i \in[k-j+1] .
$$

Lemma 2.1.16. For any $0 \leq a<b \leq 1$ and $y \in[0,1]$, we have the following identity

$$
\int_{0}^{y} u(a, t, \beta) d t=\int_{0}^{y^{\prime}} u\left(\frac{a}{b}, t, b \beta\right) d t, \quad \forall \beta \in \mathbb{R} .
$$

Here,

$$
y^{\prime}:=\frac{1}{b} u_{\beta}([0, b] \times[0, y])=\frac{1}{b} \int_{0}^{b} \int_{0}^{y} u(x, t, \beta) d t d x
$$

and $u(x, y, \beta)$ is defined in Theorem 1.2.1. 
We make some preparation before proving Lemma 2.1.16. Given $a, b \in[0,1]$, choose two sequences $\left\{a_{n}\right\}$ and $\left\{b_{n}\right\}$ such that $a_{n} \in[n], b_{n} \in[n]$ and $\lim _{n \rightarrow \infty} \frac{a_{n}}{n}=a$, $\lim _{n \rightarrow \infty} \frac{b_{n}}{n}=b$. Moreover, for any $\beta \in \mathbb{R}$, choose a sequence $\left\{q_{n}\right\}$ with $q_{n}>0$ such that $\lim _{n \rightarrow \infty} n\left(1-q_{n}\right)=\beta$.

By Lemma 2.1.5, we have

$$
\lim _{n \rightarrow \infty} \mu_{n, q_{n}}\left(\frac{\pi\left(a_{n}\right)}{n} \leq y\right)=\int_{0}^{y} u(a, t, \beta) d t
$$

We will show that

$$
\lim _{n \rightarrow \infty} \mu_{n, q_{n}}\left(\frac{\pi\left(a_{n}\right)}{n} \leq y\right)=\int_{0}^{y^{\prime}} u\left(\frac{a}{b}, t, b \beta\right) d t .
$$

Lemma 2.1.16 follows from (2.30) and (2.31). First, regarding $\left\{a_{n}\right\}$ and $\left\{b_{n}\right\}$ as fixed sequences, $y$ as a fixed number, we make the following definitions,

$$
\begin{gathered}
R_{0}:=[0, b] \times[0, y], \quad R_{1}:=[b, 1] \times[0, y], \\
K_{n}:=\left\{\left(v_{1}, v_{2}, \cdots, v_{n-b_{n}+1}\right): v_{i} \in[n] \text { and } i \neq j \Rightarrow v_{i} \neq v_{j}\right\}, \\
f_{n}(v):=\left|\left\{v_{i} \in v: v_{i} \leq n y\right\}\right| \quad \text { for } v \in K_{n}, \\
G_{n}(\lambda):=\left\{v \in K_{n}:\left|\frac{1}{n} f_{n}(v)-u_{\beta}\left(R_{1}\right)\right|<\lambda\right\} .
\end{gathered}
$$

Here $K_{n}$ consists of all possible values $\pi\left(\left[b_{n}, n\right]\right)$ can take when $\pi \in S_{n} . f_{n}\left(\pi\left(\left[b_{n}, n\right]\right)\right)$ denotes the number of points $\left(\frac{i}{n}, \frac{\pi(i)}{n}\right)$ inside the rectangle $\left[\frac{b_{n}}{n}, 1\right] \times[0, y]$.

Next we show that, for any $\lambda>0$,

$$
\lim _{n \rightarrow \infty} \mu_{n, q_{n}}\left(\pi\left(\left[b_{n}, n\right]\right) \notin G_{n}(\lambda)\right)=0
$$

Proof of (2.32). First, since the difference between $\left[\frac{b_{n}}{n}, 1\right] \times[0, y]$ and $R_{1}$ is a rectangle with width $\left|\frac{b_{n}}{n}-b\right|$, it follows that

$$
\begin{aligned}
& \left|f_{n}\left(\pi\left(\left[b_{n}, n\right]\right)\right)-n L_{\pi}\left(R_{1}\right)\right| \\
= & ||\left\{i:\left(\frac{i}{n}, \frac{\pi(i)}{n}\right) \in\left[\frac{b_{n}}{n}, 1\right] \times[0, y]\right\}|-|\left\{i:\left(\frac{i}{n}, \frac{\pi(i)}{n}\right) \in R_{1}\right\}|| \\
\leq & \left|b_{n}-n b\right|+1 .
\end{aligned}
$$


Thus, for any $\lambda>0$, there exists a $N>0$ such that for all $n>N$,

$$
\left|\frac{1}{n} f_{n}\left(\pi\left(\left[b_{n}, n\right]\right)\right)-L_{\pi}\left(R_{1}\right)\right| \leq\left|\frac{b_{n}}{n}-b\right|+\frac{1}{n}<\frac{\lambda}{2}
$$

Here we use the fact that $\lim _{n \rightarrow \infty} \frac{b_{n}}{n}=b$. Hence, for any $n>N$, we have

$$
\begin{aligned}
& \left|\frac{1}{n} f_{n}\left(\pi\left(\left[b_{n}, n\right]\right)\right)-u_{\beta}\left(R_{1}\right)\right| \geq \lambda \\
\Rightarrow & \left|\frac{1}{n} f_{n}\left(\pi\left(\left[b_{n}, n\right]\right)\right)-L_{\pi}\left(R_{1}\right)\right|+\left|L_{\pi}\left(R_{1}\right)-u_{\beta}\left(R_{1}\right)\right| \geq \lambda \\
\Rightarrow & \left|L_{\pi}\left(R_{1}\right)-u_{\beta}\left(R_{1}\right)\right|>\frac{\lambda}{2} .
\end{aligned}
$$

Thus,

$$
\begin{aligned}
& \mu_{n, q_{n}}\left(\pi\left(\left[b_{n}, n\right]\right) \notin G_{n}(\lambda)\right) \\
= & \mu_{n, q_{n}}\left(\left|\frac{1}{n} f_{n}\left(\pi\left(\left[b_{n}, n\right]\right)\right)-u_{\beta}\left(R_{1}\right)\right| \geq \lambda\right) \\
\leq & \mu_{n, q_{n}}\left(\left|L_{\pi}\left(R_{1}\right)-u_{\beta}\left(R_{1}\right)\right|>\frac{\lambda}{2}\right) .
\end{aligned}
$$

(2.32) follows from the above inequality and Lemma 2.1.6.

Next we show that, for any $\epsilon>0$, we can choose a sufficiently small $\lambda$ and $N>0$ such that for all $n>N$ and any $v \in G_{n}(\lambda)$,

$$
\left|\mu_{n, q_{n}}\left(\frac{\pi\left(a_{n}\right)}{n} \leq y \mid \pi\left(\left[b_{n}, n\right]\right)=v\right)-\int_{0}^{y^{\prime}} u\left(\frac{a}{b}, t, b \beta\right) d t\right|<\frac{\epsilon}{3} .
$$

Proof of (2.33). Assume $n$ is sufficiently large such that $a_{n}<b_{n}$. For any $v \in G_{n}(\lambda)$, here the value of $\lambda$ is to be determined, we have

$$
\begin{aligned}
& \mu_{n, q_{n}}\left(\frac{\pi\left(a_{n}\right)}{n} \leq y \mid \pi\left(\left[b_{n}, n\right]\right)=v\right) \\
= & \mu_{n, q_{n}}\left(\pi\left(a_{n}\right) \leq n y \mid \pi\left(\left[b_{n}, n\right]\right)=v\right) \\
= & \mu_{n, q_{n}}\left(\pi_{\left[1, b_{n}-1\right]}\left(a_{n}\right) \leq\lfloor n y\rfloor-f_{n}(v) \mid \pi\left(\left[b_{n}, n\right]\right)=v\right) \\
= & \mu_{b_{n}-1, q_{n}}\left(\tau\left(a_{n}\right) \leq\lfloor n y\rfloor-f_{n}(v)\right) \\
= & \mu_{b_{n}-1, q_{n}}\left(\frac{\tau\left(a_{n}\right)}{b_{n}-1} \leq \frac{1}{b_{n}-1}\left(\lfloor n y\rfloor-f_{n}(v)\right)\right)
\end{aligned}
$$




$$
=\mu_{b_{n}-1, q_{n}}\left(\frac{\tau\left(a_{n}\right)}{b_{n}-1} \leq \frac{n}{b_{n}-1}\left(\frac{\lfloor n y\rfloor}{n}-\frac{f_{n}(v)}{n}\right)\right) .
$$

The second equality follows since, conditioned on $\pi\left(\left[b_{n}, n\right]\right)=v$, we have

$$
\left\{\pi \in S_{n}: \pi\left(a_{n}\right) \leq n y\right\}=\left\{\pi \in S_{n}: \pi_{\left[1, b_{n}-1\right]}\left(a_{n}\right) \leq\lfloor n y\rfloor-f_{n}(v)\right\}
$$

Note that $\lfloor n y\rfloor-f_{n}(v)$ is the number of $i \leq n y$ which is not in $v$. The third equality is due to Lemma 1.1.5 and Lemma 1.1 .6 with $\tau \sim \mu_{b_{n}-1, q_{n}}$. Next, by the following facts,

$$
\begin{gathered}
\lim _{n \rightarrow \infty}\left(b_{n}-1\right)\left(1-q_{n}\right)=\lim _{n \rightarrow \infty} \frac{b_{n}-1}{n} \cdot \lim _{n \rightarrow \infty} n\left(1-q_{n}\right)=b \beta, \\
\lim _{n \rightarrow \infty} \frac{a_{n}}{b_{n}-1}=\lim _{n \rightarrow \infty} \frac{a_{n}}{n} \cdot \lim _{n \rightarrow \infty} \frac{n}{b_{n}-1}=\frac{a}{b}, \\
\lim _{n \rightarrow \infty} \frac{n}{b_{n}-1}\left(\frac{\lfloor n y\rfloor}{n}-u_{\beta}\left(R_{1}\right)\right)=\frac{1}{b}\left(y-u_{\beta}\left(R_{1}\right)\right)=\frac{1}{b} u_{\beta}\left(R_{0}\right)=y^{\prime},
\end{gathered}
$$

and Lemma 2.1.5, we have

$$
\lim _{n \rightarrow \infty} \mu_{b_{n}-1, q_{n}}\left(\frac{\tau\left(a_{n}\right)}{b_{n}-1} \leq \frac{n}{b_{n}-1}\left(\frac{\lfloor n y\rfloor}{n}-u_{\beta}\left(R_{1}\right)\right)\right)=\int_{0}^{y^{\prime}} u\left(\frac{a}{b}, t, b \beta\right) d t .
$$

Hence, there exists $N_{1}>0$ such that for any $n>N_{1}$,

$$
\left|\mu_{b_{n}-1, q_{n}}\left(\frac{\tau\left(a_{n}\right)}{b_{n}-1} \leq \frac{n}{b_{n}-1}\left(\frac{\lfloor n y\rfloor}{n}-u_{\beta}\left(R_{1}\right)\right)\right)-\int_{0}^{y^{\prime}} u\left(\frac{a}{b}, t, b \beta\right) d t\right|<\frac{\epsilon}{6} .
$$

By (2.37), there exists $N_{2}>0$ such that for all $n>N_{2}$,

$$
\frac{n}{b_{n}-1}<\frac{2}{b} \quad \text { and } \quad\left|\frac{n}{b_{n}-1}\left(\frac{\lfloor n y\rfloor}{n}-u_{\beta}\left(R_{1}\right)\right)-y^{\prime}\right|<\lambda \text {. }
$$

Hence, for any $n>N_{2}$ and any $v \in G_{n}(\lambda)$, we have

$$
\begin{aligned}
& \quad\left|\frac{n}{b_{n}-1}\left(\frac{\lfloor n y\rfloor}{n}-\frac{f_{n}(v)}{n}\right)-y^{\prime}\right| \\
& \leq\left|\frac{n}{b_{n}-1}\left(\frac{\lfloor n y\rfloor}{n}-\frac{f_{n}(v)}{n}\right)-\frac{n}{b_{n}-1}\left(\frac{\lfloor n y\rfloor}{n}-u_{\beta}\left(R_{1}\right)\right)\right| \\
& \quad+\left|\frac{n}{b_{n}-1}\left(\frac{\lfloor n y\rfloor}{n}-u_{\beta}\left(R_{1}\right)\right)-y^{\prime}\right| \\
& <\left(\frac{2}{b}+1\right) \lambda .
\end{aligned}
$$


Let $C:=\frac{2}{b}+1$. Since, by (2.39) and (2.40), both

$$
\frac{n}{b_{n}-1}\left(\frac{\lfloor n y\rfloor}{n}-\frac{f_{n}(v)}{n}\right) \quad \text { and } \quad \frac{n}{b_{n}-1}\left(\frac{\lfloor n y\rfloor}{n}-u_{\beta}\left(R_{1}\right)\right)
$$

are in the interval $\left(y^{\prime}-C \lambda, y^{\prime}+C \lambda\right)$, it follows that, for any $n>N_{2}$ and any $v \in$ $G_{n}(\lambda)$

$$
\begin{aligned}
\mid \mu_{b_{n}-1, q_{n}}\left(\frac{\tau\left(a_{n}\right)}{b_{n}-1}\right. & \left.\leq \frac{n}{b_{n}-1}\left(\frac{\lfloor n y\rfloor}{n}-\frac{f_{n}(v)}{n}\right)\right) \\
& -\mu_{b_{n}-1, q_{n}}\left(\frac{\tau\left(a_{n}\right)}{b_{n}-1} \leq \frac{n}{b_{n}-1}\left(\frac{\lfloor n y\rfloor}{n}-u_{\beta}\left(R_{1}\right)\right)\right) \mid \\
<\mu_{b_{n}-1, q_{n}}\left(\frac{\tau\left(a_{n}\right)}{b_{n}-1}\right. & \left.\in\left(y^{\prime}-C \lambda, y^{\prime}+C \lambda\right)\right) .
\end{aligned}
$$

By (2.35) and Lemma 2.1.8, there exists $N_{3}>0$ such that for all $n>N_{3}$,

$$
\mu_{b_{n}-1, q_{n}}\left(\frac{\tau\left(a_{n}\right)}{b_{n}-1} \in\left(y^{\prime}-C \lambda, y^{\prime}+C \lambda\right)\right)<4 C \lambda e^{b|\beta|}
$$

Therefore, we can fix $\lambda=\frac{\epsilon}{24 C} e^{-b|\beta|}$ in the first place. Then, by (2.38), (2.41) and (2.42), for any $n>\max \left(N_{1}, N_{2}, N_{3}\right)$ and any $v \in G_{n}(\lambda)$,

$$
\begin{aligned}
& \left|\mu_{b_{n}-1, q_{n}}\left(\frac{\tau\left(a_{n}\right)}{b_{n}-1} \leq \frac{n}{b_{n}-1}\left(\frac{\lfloor n y\rfloor}{n}-\frac{f_{n}(v)}{n}\right)\right)-\int_{0}^{y^{\prime}} u\left(\frac{a}{b}, t, b \beta\right) d t\right| \\
< & \frac{\epsilon}{6}+\frac{\epsilon}{6}=\frac{\epsilon}{3} .
\end{aligned}
$$

(2.33) follows by (2.34) and (2.43).

Now we are in the position to prove (2.31), which completes the proof of Lemma 2.1.16.

Proof of (2.31). For simplicity, let $I:=\int_{0}^{y^{\prime}} u\left(\frac{a}{b}, t, b \beta\right) d t$. Since

$$
y^{\prime}=\frac{1}{b} u_{\beta}\left(R_{0}\right) \leq \frac{1}{b} u_{\beta}([0, b] \times[0,1])=1,
$$

we have

$$
I=\int_{0}^{y^{\prime}} u\left(\frac{a}{b}, t, b \beta\right) d t \leq \int_{0}^{1} u\left(\frac{a}{b}, t, b \beta\right) d t=1 .
$$

Then, given $\epsilon>0$, fix the value of $\lambda$ such that (2.33) holds for any $n>N_{1}$ and any $v \in G_{n}(\lambda)$. By (2.32), there exists $N_{2}>0$ such that for any $n>N_{2}$,

$$
\mu_{n, q_{n}}\left(\pi\left(\left[b_{n}, n\right]\right) \notin G_{n}(\lambda)\right)<\frac{\epsilon}{3} .
$$


Then, for any $n>\max \left(N_{1}, N_{2}\right)$,

$$
\begin{aligned}
& \quad\left|\mu_{n, q_{n}}\left(\frac{\pi\left(a_{n}\right)}{n} \leq y\right)-I\right| \\
& =\mid \sum_{v \in K_{n}} \mu_{n, q_{n}}\left(\frac{\pi\left(a_{n}\right)}{n} \leq y \mid \pi\left(\left[b_{n}, n\right]\right)=v\right) \cdot \mu_{n, q_{n}}\left(\pi\left(\left[b_{n}, n\right]\right)=v\right) \\
& \quad-\sum_{v \in K_{n}} I \cdot \mu_{n, q_{n}}\left(\pi\left(\left[b_{n}, n\right]\right)=v\right) \mid \\
& \leq \sum_{v \in G_{n}(\lambda)\left|\mu_{n, q_{n}}\left(\frac{\pi\left(a_{n}\right)}{n} \leq y \mid \pi\left(\left[b_{n}, n\right]\right)=v\right)-I\right| \cdot \mu_{n, q_{n}}\left(\pi\left(\left[b_{n}, n\right]\right)=v\right)} \quad+\sum_{v \notin G_{n}(\lambda)} \mu_{n, q_{n}}\left(\frac{\pi\left(a_{n}\right)}{n} \leq y \mid \pi\left(\left[b_{n}, n\right]\right)=v\right) \cdot \mu_{n, q_{n}}\left(\pi\left(\left[b_{n}, n\right]\right)=v\right) \\
& \quad+\sum_{v \notin G_{n}(\lambda)} I \cdot \mu_{n, q_{n}}\left(\pi\left(\left[b_{n}, n\right]\right)=v\right) \\
& \leq \frac{\epsilon}{3} \cdot \sum_{v \in G_{n}(\lambda)} \mu_{n, q_{n}}\left(\pi\left(\left[b_{n}, n\right]\right)=v\right)+2 \cdot \sum_{v \notin G_{n}(\lambda)} \mu_{n, q_{n}}\left(\pi\left(\left[b_{n}, n\right]\right)=v\right) \\
& <\frac{\epsilon}{3}+\frac{2 \epsilon}{3}=\epsilon .
\end{aligned}
$$

Here we use (2.33) and (2.44) in the second to last inequality.

Lemma 2.1.17. For any $0 \leq a<b \leq 1$ and any $\beta \in \mathbb{R}$, suppose we have sequences $\left\{a_{n}\right\},\left\{b_{n}\right\}$ and $\left\{q_{n}\right\}$ such that $a_{n} \in[n], b_{n} \in[n], q_{n}>0$ and

$$
\lim _{n \rightarrow \infty} \frac{a_{n}}{n}=a, \quad \lim _{n \rightarrow \infty} \frac{b_{n}}{n}=b, \quad \lim _{n \rightarrow \infty} n\left(1-q_{n}\right)=\beta
$$

Then, for any $A=\left[y_{1}, y_{2}\right] \subset[0,1]$ and $B=\left[y_{3}, y_{4}\right] \subset[0,1]$,

$$
\lim _{n \rightarrow \infty} \mu_{n, q_{n}}\left(\mathbb{1}_{A}\left(\frac{\pi\left(a_{n}\right)}{n}\right) \mathbb{1}_{B}\left(\frac{\pi\left(b_{n}\right)}{n}\right)\right)-\mu_{n, q_{n}}\left(\mathbb{1}_{A}\left(\frac{\pi\left(a_{n}\right)}{n}\right)\right) \mu_{n, q_{n}}\left(\mathbb{1}_{B}\left(\frac{\pi\left(b_{n}\right)}{n}\right)\right)=0
$$

Proof. The proof is similar to the proof of Lemma 2.1.16, and we inherit those definitions in the previous proof. First of all, since

$$
\begin{aligned}
& \mu_{n, q_{n}}\left(\mathbb{1}_{A}\left(\frac{\pi\left(a_{n}\right)}{n}\right) \mathbb{1}_{B}\left(\frac{\pi\left(b_{n}\right)}{n}\right)\right) \\
= & \mu_{n, q_{n}}\left(\mathbb{1}_{\left[0, y_{2}\right]}\left(\frac{\pi\left(a_{n}\right)}{n}\right) \mathbb{1}_{B}\left(\frac{\pi\left(b_{n}\right)}{n}\right)\right)-\mu_{n, q_{n}}\left(\mathbb{1}_{\left[0, y_{1}\right)}\left(\frac{\pi\left(a_{n}\right)}{n}\right) \mathbb{1}_{B}\left(\frac{\pi\left(b_{n}\right)}{n}\right)\right)
\end{aligned}
$$

and

$$
\mu_{n, q_{n}}\left(\mathbb{1}_{A}\left(\frac{\pi\left(a_{n}\right)}{n}\right)\right)=\mu_{n, q_{n}}\left(\mathbb{1}_{\left[0, y_{2}\right]}\left(\frac{\pi\left(a_{n}\right)}{n}\right)\right)-\mu_{n, q_{n}}\left(\mathbb{1}_{\left[0, y_{1}\right)}\left(\frac{\pi\left(a_{n}\right)}{n}\right)\right)
$$


it suffices to show the cases when the interval $A$ is of the form $[0, y]$ or $[0, y)$ for any $y \in[0,1]$. Moreover, by Lemma 2.1.5, we have

$$
\lim _{n \rightarrow \infty} \mu_{n, q_{n}}\left(\frac{\pi\left(a_{n}\right)}{n}=y\right)=0, \quad \forall y \in[0,1] .
$$

Hence, it suffices to show the case when $A=[0, y]$, for any $y \in[0,1]$.

By Lemma 2.1.16, define

$$
I:=\int_{0}^{y} u(a, t, \beta) d t=\int_{0}^{y^{\prime}} u\left(\frac{a}{b}, t, b \beta\right) d t .
$$

By Lemma 2.1.5, we have

$$
\lim _{n \rightarrow \infty} \mu_{n, q_{n}}\left(\mathbb{1}_{A}\left(\frac{\pi\left(a_{n}\right)}{n}\right)\right)=\int_{0}^{y} u(a, t, \beta) d t=I .
$$

Hence it suffices to show the following,

$$
\lim _{n \rightarrow \infty} \mu_{n, q_{n}}\left(\mathbb{1}\left(\frac{\pi\left(a_{n}\right)}{n} \leq y\right) \mathbb{1}_{B}\left(\frac{\pi\left(b_{n}\right)}{n}\right)\right)-\mu_{n, q_{n}}\left(\mathbb{1}_{B}\left(\frac{\pi\left(b_{n}\right)}{n}\right)\right) \cdot I=0
$$

for any $y \in[0,1]$. Given $\epsilon>0$, by (2.33), there exists $\lambda>0$ and $N_{1}>0$ such that for any $n>N_{1}$ and any $v \in G_{n}(\lambda)$,

$$
\left|\mu_{n, q_{n}}\left(\frac{\pi\left(a_{n}\right)}{n} \leq y \mid \pi\left(\left[b_{n}, n\right]\right)=v\right)-I\right|<\frac{\epsilon}{3}
$$

By (2.32), there exists $N_{2}>0$ such that for any $n>N_{2}$,

$$
\mu_{n, q_{n}}\left(\pi\left(\left[b_{n}, n\right]\right) \notin G_{n}(\lambda)\right)<\frac{\epsilon}{3} .
$$

Moreover, by conditioning on the value of $\pi\left(\left[b_{n}, n\right]\right)$, we have

$$
\begin{aligned}
& \mu_{n, q_{n}}\left(\mathbb{1}\left(\frac{\pi\left(a_{n}\right)}{n} \leq y\right) \mathbb{1}_{B}\left(\frac{\pi\left(b_{n}\right)}{n}\right)\right) \\
= & \sum_{v \in K_{n}} \mu_{n, q_{n}}\left(\mathbb{1}\left(\frac{\pi\left(a_{n}\right)}{n} \leq y\right) \mathbb{1}_{B}\left(\frac{\pi\left(b_{n}\right)}{n}\right) \mid \pi\left(\left[b_{n}, n\right]\right)=v\right) \cdot \mu_{n, q_{n}}\left(\pi\left(\left[b_{n}, n\right]\right)=v\right) \\
= & \sum_{v \in K_{n}} \mu_{n, q_{n}}\left(\mathbb{1}\left(\frac{\pi\left(a_{n}\right)}{n} \leq y\right) \mid \pi\left(\left[b_{n}, n\right]\right)=v\right) \cdot \mathbb{1}_{B}\left(\frac{v_{1}}{n}\right) \cdot \mu_{n, q_{n}}\left(\pi\left(\left[b_{n}, n\right]\right)=v\right) \\
= & \sum_{v \in G_{n}(\lambda)} \mu_{n, q_{n}}\left(\mathbb{1}\left(\frac{\pi\left(a_{n}\right)}{n} \leq y\right) \mid \pi\left(\left[b_{n}, n\right]\right)=v\right) \cdot \mathbb{1}_{B}\left(\frac{v_{1}}{n}\right) \cdot \mu_{n, q_{n}}\left(\pi\left(\left[b_{n}, n\right]\right)=v\right) \\
+ & \sum_{v \notin G_{n}(\lambda)} \mu_{n, q_{n}}\left(\mathbb{1}\left(\frac{\pi\left(a_{n}\right)}{n} \leq y\right) \mid \pi\left(\left[b_{n}, n\right]\right)=v\right) \cdot \mathbb{1}_{B}\left(\frac{v_{1}}{n}\right) \cdot \mu_{n, q_{n}}\left(\pi\left(\left[b_{n}, n\right]\right)=v\right)
\end{aligned}
$$


and

$$
\begin{aligned}
& \mu_{n, q_{n}}\left(\mathbb{1}_{B}\left(\frac{\pi\left(b_{n}\right)}{n}\right)\right) \\
= & \sum_{v \in K_{n}} \mu_{n, q_{n}}\left(\mathbb{1}_{B}\left(\frac{\pi\left(b_{n}\right)}{n}\right) \mid \pi\left(\left[b_{n}, n\right]\right)=v\right) \cdot \mu_{n, q_{n}}\left(\pi\left(\left[b_{n}, n\right]\right)=v\right) \\
= & \sum_{v \in K_{n}} \mathbb{1}_{B}\left(\frac{v_{1}}{n}\right) \cdot \mu_{n, q_{n}}\left(\pi\left(\left[b_{n}, n\right]\right)=v\right) \\
= & \sum_{v \in G_{n}(\lambda)} \mathbb{1}_{B}\left(\frac{v_{1}}{n}\right) \cdot \mu_{n, q_{n}}\left(\pi\left(\left[b_{n}, n\right]\right)=v\right) \\
& \quad+\sum_{v \notin G_{n}(\lambda)} \mathbb{1}_{B}\left(\frac{v_{1}}{n}\right) \cdot \mu_{n, q_{n}}\left(\pi\left(\left[b_{n}, n\right]\right)=v\right) .
\end{aligned}
$$

Here $v_{1}$ denotes the first entry of vector $v$. Hence, for any $n>\max \left(N_{1}, N_{2}\right)$, we have

$$
\begin{aligned}
& \left|\mu_{n, q_{n}}\left(\mathbb{1}\left(\frac{\pi\left(a_{n}\right)}{n} \leq y\right) \mathbb{1}_{B}\left(\frac{\pi\left(b_{n}\right)}{n}\right)\right)-\mu_{n, q_{n}}\left(\mathbb{1}_{B}\left(\frac{\pi\left(b_{n}\right)}{n}\right)\right) \cdot I\right| \\
\leq & \sum_{v \in G_{n}(\lambda)}\left|\mu_{n, q_{n}}\left(\mathbb{1}\left(\frac{\pi\left(a_{n}\right)}{n} \leq y\right) \mid \pi\left(\left[b_{n}, n\right]\right)=v\right)-I\right| \cdot \mu_{n, q_{n}}\left(\pi\left(\left[b_{n}, n\right]\right)=v\right) \\
& +2 \sum_{v \notin G_{n}(\lambda)} \mu_{n, q_{n}}\left(\pi\left(\left[b_{n}, n\right]\right)=v\right) \\
\leq & \frac{\epsilon}{3} \sum_{v \in G_{n}(\lambda)} \mu_{n, q_{n}}\left(\pi\left(\left[b_{n}, n\right]\right)=v\right)+2 \sum_{v \notin G_{n}(\lambda)} \mu_{n, q_{n}}\left(\pi\left(\left[b_{n}, n\right]\right)=v\right) \\
< & \frac{\epsilon}{3}+2 \cdot \frac{\epsilon}{3}=\epsilon .
\end{aligned}
$$

The first inequality follows from triangle inequality and the fact that,

$$
\mu_{n, q_{n}}\left(\mathbb{1}\left(\frac{\pi\left(a_{n}\right)}{n} \leq y\right) \mid \pi\left(\left[b_{n}, n\right]\right)=v\right) \leq 1, \quad \mathbb{1}_{B}\left(\frac{v_{1}}{n}\right) \leq 1, \text { and } I \leq 1
$$

The last two inequalities follow from (2.46) and (2.47) respectively.

Before we start to prove Lemma 2.1.1 and Lemma 2.1.2, we briefly introduce the following facts:

Lemma 2.1.18. For any $s, t, i \in[n]$,

$$
\min \left(q^{d}, q^{-d}\right) \leq \frac{\mu_{n, q}(\pi(s)=i)}{\mu_{n, q}(\pi(t)=i)} \leq \max \left(q^{d}, q^{-d}\right)
$$

where $d=|s-t|$. 
Lemma 2.1.19. For any $s, t, w, i, j \in[n]$ such that $i \neq j$ and either $w<\min (s, t)$ or $w>\max (s, t)$,

$$
\min \left(q^{d}, q^{-d}\right) \leq \frac{\mu_{n, q}\left(\left\{\pi \in S_{n}: \pi(s)=i \text { and } \pi(w)=j\right\}\right)}{\mu_{n, q}\left(\left\{\pi \in S_{n}: \pi(t)=i \text { and } \pi(w)=j\right\}\right)} \leq \max \left(q^{d}, q^{-d}\right),
$$

where $d=|s-t|$.

Lemma 2.1.18 and Lemma 2.1.19 follow from similar argument as in the proof of Lemma 2.1.7. We omit their proofs. From these two lemmas, we can show the following,

Lemma 2.1.20. For any $A \subset[0,1]$ and any $s, t \in[n]$,

$$
\left|\mu_{n, q}\left(\mathbb{1}_{A}\left(\frac{\pi(s)}{n}\right)\right)-\mu_{n, q}\left(\mathbb{1}_{A}\left(\frac{\pi(t)}{n}\right)\right)\right| \leq M
$$

where $M=\max \left(\left|1-q^{d}\right|,\left|1-q^{-d}\right|\right)$ and $d=|s-t|$.

Lemma 2.1.21. For any $A, B \subset[0,1]$ and any $s, t, w \in[n]$ such that either $w<$ $\min (s, t)$ or $w>\max (s, t)$,

$$
\left|\mu_{n, q}\left(\mathbb{1}_{A}\left(\frac{\pi(s)}{n}\right) \mathbb{1}_{B}\left(\frac{\pi(w)}{n}\right)\right)-\mu_{n, q}\left(\mathbb{1}_{A}\left(\frac{\pi(t)}{n}\right) \mathbb{1}_{B}\left(\frac{\pi(w)}{n}\right)\right)\right| \leq M
$$

where $M=\max \left(\left|1-q^{d}\right|,\left|1-q^{-d}\right|\right)$ and $d=|s-t|$.

Here we only deduce Lemma 2.1.20 from Lemma 2.1.18. Lemma 2.1.21 follows from Lemma 2.1.19 by the similar argument.

Proof of Lemma 2.1.20. Without loss of generality, assume $0<q<1$. By Lemma 2.1.18, for any $i \in[n]$, we have

$$
q^{d} \leq \frac{\mu_{n, q}(\pi(s)=i)}{\mu_{n, q}(\pi(t)=i)} \leq q^{-d}
$$

Hence

$$
q^{d} \sum_{\left\{i: \frac{i}{n} \in A\right\}} \mu_{n, q}(\pi(t)=i) \leq \sum_{\left\{i: \frac{i}{n} \in A\right\}} \mu_{n, q}(\pi(s)=i) \leq q^{-d} \sum_{\left\{i: \frac{i}{n} \in A\right\}} \mu_{n, q}(\pi(t)=i)
$$


Thus

$$
\mu_{n, q}\left(\mathbb{1}_{A}\left(\frac{\pi(t)}{n}\right)\right) \cdot q^{d} \leq \mu_{n, q}\left(\mathbb{1}_{A}\left(\frac{\pi(s)}{n}\right)\right) \leq \mu_{n, q}\left(\mathbb{1}_{A}\left(\frac{\pi(t)}{n}\right)\right) \cdot q^{-d}
$$

Therefore

$$
\begin{aligned}
& \left|\mu_{n, q}\left(\mathbb{1}_{A}\left(\frac{\pi(s)}{n}\right)\right)-\mu_{n, q}\left(\mathbb{1}_{A}\left(\frac{\pi(t)}{n}\right)\right)\right| \\
\leq & \mu_{n, q}\left(\mathbb{1}_{A}\left(\frac{\pi(t)}{n}\right)\right) \max \left(q^{-d}-1,1-q^{d}\right) \\
\leq & \max \left(q^{-d}-1,1-q^{d}\right) .
\end{aligned}
$$

Proof of Lemma 2.1.2. Let $m$ be a positive integer whose value is to be determined. Define the following $m+1$ sequences $\left\{a_{n}^{(k)}\right\}, 0 \leq k \leq m$, as follows,

$$
a_{n}^{(k)}:= \begin{cases}1, & \text { if } k=0 \\ \left\lceil\frac{k n}{m}\right\rceil, & \text { if } 1 \leq k \leq m .\end{cases}
$$

Then, for any $0 \leq k \leq m$, we have $\lim _{n \rightarrow \infty} \frac{a_{n}^{(k)}}{n}=\frac{k}{m}$. Also, for any $0 \leq k \leq m-1$ and $n>m$ we have

$$
1 \leq a_{n}^{(k+1)}-a_{n}^{(k)} \leq \frac{n}{m}+1
$$

Then, for any $n>m$ and any $i, j \in[n]$ with $i<j$, there exist unique $k$ and $l$ such that

$$
i \in\left[a_{n}^{(k)}, a_{n}^{(k+1)}\right), \quad \text { and } \quad j \in\left(a_{n}^{(l-1)}, a_{n}^{(l)}\right] .
$$

Clearly, we have

$$
k<l, \quad\left|i-a_{n}^{(k)}\right| \leq \frac{n}{m} \quad \text { and } \quad\left|j-a_{n}^{(l)}\right| \leq \frac{n}{m}
$$

Then, given $\epsilon>0$, fix a sufficiently large $m$ in the first place such that,

$$
\left|e^{\frac{\beta}{m}}-1\right|<\frac{\epsilon}{12}, \quad\left|e^{-\frac{\beta}{m}}-1\right|<\frac{\epsilon}{12}
$$

Next, since $\lim _{n \rightarrow \infty} q_{n}^{n}=e^{-\beta}$, there exists $N_{1}>0$ such that for any $n>N_{1}$,

$$
\left|e^{\frac{\beta}{m}}-q_{n}^{-\frac{n}{m}}\right|<\frac{\epsilon}{12}, \quad\left|e^{-\frac{\beta}{m}}-q_{n}^{\frac{n}{m}}\right|<\frac{\epsilon}{12} .
$$


Then, by triangle inequality, for any $n>N_{1}$,

$$
\max \left(\left|1-q_{n}^{\frac{n}{m}}\right|,\left|1-q_{n}^{-\frac{n}{m}}\right|\right)<\frac{\epsilon}{6}
$$

For the simplicity of notation, define

$$
\begin{aligned}
U & :=\left|\mu_{n, q_{n}}\left(\mathbb{1}_{A}\left(\frac{\pi(i)}{n}\right)\right) \mu_{n, q_{n}}\left(\mathbb{1}_{B}\left(\frac{\pi(j)}{n}\right)\right)-\mu_{n, q_{n}}\left(\mathbb{1}_{A}\left(\frac{\pi\left(a_{n}^{(k)}\right)}{n}\right)\right) \mu_{n, q_{n}}\left(\mathbb{1}_{B}\left(\frac{\pi(j)}{n}\right)\right)\right|, \\
V & :=\left|\mu_{n, q_{n}}\left(\mathbb{1}_{A}\left(\frac{\pi(i)}{n}\right) \mathbb{1}_{B}\left(\frac{\pi(j)}{n}\right)\right)-\mu_{n, q_{n}}\left(\mathbb{1}_{A}\left(\frac{\pi\left(a_{n}^{(k)}\right)}{n}\right) \mathbb{1}_{B}\left(\frac{\pi(j)}{n}\right)\right)\right|, \\
W & :=\left|\mu_{n, q_{n}}\left(\mathbb{1}_{A}\left(\frac{\pi\left(a_{n}^{(k)}\right)}{n}\right) \mathbb{1}_{B}\left(\frac{\pi(j)}{n}\right)\right)-\mu_{n, q_{n}}\left(\mathbb{1}_{A}\left(\frac{\pi\left(a_{n}^{(k)}\right)}{n}\right)\right) \mu_{n, q_{n}}\left(\mathbb{1}_{B}\left(\frac{\pi(j)}{n}\right)\right)\right| \\
& =\left|\operatorname{Cov}_{n}\left(\mathbb{1}_{A}\left(\frac{\pi\left(a_{n}^{(k)}\right)}{n}\right), \mathbb{1}_{B}\left(\frac{\pi(j)}{n}\right)\right)\right| .
\end{aligned}
$$

Then, by (2.50), (2.51), Lemma 2.1.20 and Lemma 2.1.21, for any $n>\max \left(m, N_{1}\right)$ and any $0 \leq i<j \leq n$ with corresponding $k, l$ defined in (2.49), we have

$$
\begin{aligned}
U & =\mu_{n, q_{n}}\left(\mathbb{1}_{B}\left(\frac{\pi(j)}{n}\right)\right) \cdot\left|\mu_{n, q_{n}}\left(\mathbb{1}_{A}\left(\frac{\pi(i)}{n}\right)\right)-\mu_{n, q_{n}}\left(\mathbb{1}_{A}\left(\frac{\pi\left(a_{n}^{(k)}\right)}{n}\right)\right)\right| \\
& \leq\left|\mu_{n, q_{n}}\left(\mathbb{1}_{A}\left(\frac{\pi(i)}{n}\right)\right)-\mu_{n, q_{n}}\left(\mathbb{1}_{A}\left(\frac{\pi\left(a_{n}^{(k)}\right)}{n}\right)\right)\right| \\
& \leq \max \left(\left|1-q_{n}^{\frac{n}{m}}\right|,\left|1-q_{n}^{-\frac{n}{m}}\right|\right)<\frac{\epsilon}{6}, \\
V & \leq \max \left(\left|1-q_{n}^{\frac{n}{m}}\right|,\left|1-q_{n}^{-\frac{n}{m}}\right|\right)<\frac{\epsilon}{6} .
\end{aligned}
$$

Whence, again, by triangle inequality, for any $n>\max \left(m, N_{1}\right)$,

$$
\begin{aligned}
& \left|\operatorname{Cov}_{n}\left(\mathbb{1}_{A}\left(\frac{\pi(i)}{n}\right), \mathbb{1}_{B}\left(\frac{\pi(j)}{n}\right)\right)\right| \\
= & \left|\mu_{n, q_{n}}\left(\mathbb{1}_{A}\left(\frac{\pi(i)}{n}\right) \mathbb{1}_{B}\left(\frac{\pi(j)}{n}\right)\right)-\mu_{n, q_{n}}\left(\mathbb{1}_{A}\left(\frac{\pi(i)}{n}\right)\right) \mu_{n, q_{n}}\left(\mathbb{1}_{B}\left(\frac{\pi(j)}{n}\right)\right)\right| \\
< & U+V+W \\
< & \left|\operatorname{Cov}_{n}\left(\mathbb{1}_{A}\left(\frac{\pi\left(a_{n}^{(k)}\right)}{n}\right), \mathbb{1}_{B}\left(\frac{\pi(j)}{n}\right)\right)\right|+\frac{\epsilon}{3}
\end{aligned}
$$

By the same argument, it follows that for any $n>\max \left(m, N_{1}\right)$,

$$
\begin{aligned}
& \left|\operatorname{Cov}_{n}\left(\mathbb{1}_{A}\left(\frac{\pi\left(a_{n}^{(k)}\right)}{n}\right), \mathbb{1}_{B}\left(\frac{\pi(j)}{n}\right)\right)\right| \\
< & \left|\operatorname{Cov}_{n}\left(\mathbb{1}_{A}\left(\frac{\pi\left(a_{n}^{(k)}\right)}{n}\right), \mathbb{1}_{B}\left(\frac{\pi\left(a_{n}^{(l)}\right)}{n}\right)\right)\right|+\frac{\epsilon}{3} .
\end{aligned}
$$


Combining (2.52) and (2.53), for any $n>\max \left(m, N_{1}\right)$ and any $0 \leq i<j \leq n$ with corresponding $k, l$ defined in (2.49), we have

$$
\begin{aligned}
& \left|\operatorname{Cov}_{n}\left(\mathbb{1}_{A}\left(\frac{\pi(i)}{n}\right), \mathbb{1}_{B}\left(\frac{\pi(j)}{n}\right)\right)\right| \\
< & \left|\operatorname{Cov}_{n}\left(\mathbb{1}_{A}\left(\frac{\pi\left(a_{n}^{(k)}\right)}{n}\right), \mathbb{1}_{B}\left(\frac{\pi\left(a_{n}^{(l)}\right)}{n}\right)\right)\right|+\frac{2 \epsilon}{3} .
\end{aligned}
$$

Moreover, since $m$ is fixed, by Lemma 2.1.17, there exists $N_{2}>0$ such that, for any $n>N_{2}$ and any $0 \leq k<l \leq m$, we have

$$
\left|\operatorname{Cov}_{n}\left(\mathbb{1}_{A}\left(\frac{\pi\left(a_{n}^{(k)}\right)}{n}\right), \mathbb{1}_{B}\left(\frac{\pi\left(a_{n}^{(l)}\right)}{n}\right)\right)\right|<\frac{\epsilon}{3}
$$

Thus, for $n>\max \left(m, N_{1}, N_{2}\right)$ and any $0 \leq i<j \leq n$,

$$
\left|\mu_{n, q_{n}}\left(\mathbb{1}_{A}\left(\frac{\pi(i)}{n}\right) \mathbb{1}_{B}\left(\frac{\pi(j)}{n}\right)\right)-\mu_{n, q_{n}}\left(\mathbb{1}_{A}\left(\frac{\pi(i)}{n}\right)\right) \mu_{n, q_{n}}\left(\mathbb{1}_{B}\left(\frac{\pi(j)}{n}\right)\right)\right|<\epsilon
$$

Proof of Lemma 2.1.1. The proof of Lemma 2.1.1 is similar to the proof of Lemma 2.1.2. Firstly, since $u(x, y, \beta)$ is uniformly continuous on $[0,1] \times[0,1]$, given $\epsilon>0$, there exists $m_{1}>0$ such that

$$
\sup _{\substack{|s-t|<\frac{1}{m_{1}} \\ s, t, y \in[0,1]}}|u(s, y, \beta)-u(t, y, \beta)|<\frac{\epsilon}{6} .
$$

Hence, for any $|s-t|<\frac{1}{m_{1}}$ with $s, t \in[0,1]$, we have

$$
\begin{aligned}
& \left|\int_{y_{1}}^{y_{2}} u(s, y, \beta) d y-\int_{y_{1}}^{y_{2}} u(t, y, \beta) d y\right| \\
\leq & \int_{y_{1}}^{y_{2}}|u(s, y, \beta)-u(t, y, \beta)| d y \\
\leq & \int_{0}^{1}|u(s, y, \beta)-u(t, y, \beta)| d y \\
< & \frac{\epsilon}{6} .
\end{aligned}
$$

Then, choose an $m>2 m_{1}$ such that

$$
\left|e^{\frac{\beta}{m}}-1\right|<\frac{\epsilon}{12}, \quad\left|e^{-\frac{\beta}{m}}-1\right|<\frac{\epsilon}{12}
$$


Next, since $\lim _{n \rightarrow \infty} q_{n}^{n}=e^{-\beta}$, there exists $N_{1}>0$ such that for any $n>N_{1}$,

$$
\left|e^{\frac{\beta}{m}}-q_{n}^{-\frac{n}{m}}\right|<\frac{\epsilon}{12}, \quad\left|e^{-\frac{\beta}{m}}-q_{n}^{\frac{n}{m}}\right|<\frac{\epsilon}{12} .
$$

By triangle inequality, for any $n>N_{1}$,

$$
\max \left(\left|1-q_{n}^{\frac{n}{m}}\right|,\left|1-q_{n}^{-\frac{n}{m}}\right|\right)<\frac{\epsilon}{6}
$$

Next, define the $m+1$ sequences $\left\{a_{n}^{(k)}\right\}, 0 \leq k \leq m$, as in (2.48). By (2.50) and Lemma 2.1.20, for any $n>\max \left(m, N_{1}\right)$ and any $i \in[n]$ with corresponding $k$ defined in (2.49), we have

$$
\begin{aligned}
& \left|\mu_{n, q_{n}}\left(\mathbb{1}_{A}\left(\frac{\pi(i)}{n}\right)\right)-\mu_{n, q_{n}}\left(\mathbb{1}_{A}\left(\frac{\pi\left(a_{n}^{(k)}\right)}{n}\right)\right)\right| \\
\leq & \max \left(\left|1-q_{n}^{\frac{n}{m}}\right|,\left|1-q_{n}^{-\frac{n}{m}}\right|\right) \\
< & \frac{\epsilon}{6} .
\end{aligned}
$$

Secondly, by the definition of $a_{n}^{(k)}$ in (2.48), it is easily seen that

$$
\frac{k n}{m} \leq a_{n}^{(k)} \leq \frac{k n}{m}+1
$$

Thus, for any $n>m$ and any $i \in[n]$ with corresponding $k$ defined in (2.49), we have

$$
\begin{aligned}
& \frac{k n}{m} \leq a_{n}^{(k)} \leq i<a_{n}^{(k+1)} \leq \frac{(k+1) n}{m}+1 \\
\Rightarrow & \frac{k}{m} \leq \frac{i}{n} \leq \frac{k+1}{m}+\frac{1}{n} \\
\Rightarrow & \left|\frac{i}{n}-\frac{k}{m}\right| \leq \frac{1}{m}+\frac{1}{n}<\frac{2}{m}<\frac{1}{m_{1}} .
\end{aligned}
$$

Hence, by (2.54), for any $n>m$ and any $i \in[n]$ with corresponding $k$ defined in (2.49), we have

$$
\left|\int_{y_{1}}^{y_{2}} u\left(\frac{i}{n}, y, \beta\right) d y-\int_{y_{1}}^{y_{2}} u\left(\frac{k}{m}, y, \beta\right) d y\right|<\frac{\epsilon}{6} .
$$

Thirdly, since $\lim _{n \rightarrow \infty} \frac{a_{n}^{(k)}}{n}=\frac{k}{m}$ for any $0 \leq k \leq m$, by Lemma 2.1.5, there exists $N_{1}>0$ such that, for any $n>N_{1}$ and any $0 \leq k \leq m$,

$$
\left|\mu_{n, q_{n}}\left(\mathbb{1}_{A}\left(\frac{\pi\left(a_{n}^{(k)}\right)}{n}\right)\right)-\int_{y_{1}}^{y_{2}} u\left(\frac{k}{m}, y, \beta\right) d y\right|<\frac{\epsilon}{3} .
$$


Therefore, for any $n>\max \left(m, N_{1}, N_{2}\right)$ and any $i \in[n]$ with corresponding $k$ defined in (2.49), we have

$$
\begin{aligned}
& \mid \mu_{n, q_{n}}\left(\mathbb{1}_{A}\left(\frac{\pi(i)}{n}\right)\right)- \\
\leq \mid \mu_{n, q_{n}}\left(\mathbb{1}_{A}\left(\frac{\pi(i)}{n}\right)\right)- & \mu_{n, q_{n}}\left(\mathbb{1}_{A}\left(\frac{\pi\left(a_{n}^{(k)}\right)}{n}\right)\right) \mid \\
& +\left|\mu_{n, q_{n}}\left(\mathbb{1}_{A}\left(\frac{\pi\left(a_{n}^{(k)}\right)}{n}\right)\right)-\int_{y_{1}}^{y_{2}} u\left(\frac{k}{m}, y, \beta\right) d y\right| \\
& +\left|\int_{y_{1}}^{y_{2}} u\left(\frac{k}{m}, y, \beta\right) d y-\int_{y_{1}}^{y_{2}} u\left(\frac{i}{n}, y, \beta\right) d y\right| \\
<\frac{\epsilon}{3}+\frac{\epsilon}{6}+\frac{\epsilon}{6}<\epsilon . &
\end{aligned}
$$

The last inequality follows from (2.55), (2.56) and (2.57).

\subsection{Proof of Theorem 1}

In this section, we show Theorem 1 using Lemma 2.1.1 and Lemma 2.1.2. In the proof we approximate the continuous function $f$ on $[0,1]$ by a sequence of simple functions. The following elementary lemma will be used in the proof.

Lemma 2.2.1. Given random variables $X, X^{\prime}, Y, Y^{\prime}$ such that $\left|X-X^{\prime}\right|<\epsilon,\left|Y-Y^{\prime}\right|<\epsilon$ and $\max \left(|X|,\left|X^{\prime}\right|,|Y|,\left|Y^{\prime}\right|\right)<M$, we have

$$
\left|\operatorname{Cov}(X, Y)-\operatorname{Cov}\left(X^{\prime}, Y^{\prime}\right)\right|<4 M \epsilon
$$

Proof. Since $X Y-X^{\prime} Y^{\prime}=X\left(Y-Y^{\prime}\right)+Y^{\prime}\left(X-X^{\prime}\right)$, we have

$$
\left|X Y-X^{\prime} Y^{\prime}\right| \leq|X|\left|Y-Y^{\prime}\right|+\left|Y^{\prime}\right|\left|X-X^{\prime}\right|<2 M \epsilon
$$

Similarly, since $\mathbb{E}(X) \mathbb{E}(Y)-\mathbb{E}\left(X^{\prime}\right) \mathbb{E}\left(Y^{\prime}\right)=\mathbb{E}(X) \mathbb{E}\left(Y-Y^{\prime}\right)+\mathbb{E}\left(Y^{\prime}\right) \mathbb{E}\left(X-X^{\prime}\right)$, we have

$$
\left|\mathbb{E}(X) \mathbb{E}(Y)-\mathbb{E}\left(X^{\prime}\right) \mathbb{E}\left(Y^{\prime}\right)\right|<2 M \epsilon
$$

Hence

$$
\begin{aligned}
\left|\operatorname{Cov}(X, Y)-\operatorname{Cov}\left(X^{\prime}, Y^{\prime}\right)\right| & \leq \mathbb{E}\left|X Y-X^{\prime} Y^{\prime}\right|+\left|\mathbb{E}(X) \mathbb{E}(Y)-\mathbb{E}\left(X^{\prime}\right) \mathbb{E}\left(Y^{\prime}\right)\right| \\
& <4 M \epsilon
\end{aligned}
$$


Proof of Theorem 1. Given continuous function $f:[0,1] \longrightarrow \mathbb{R}$, define a sequence of simple functions $\left\{g_{m}\right\}_{m \geq 1}$ as follows,

$$
g_{m}(x):=\sum_{k=1}^{m} f\left(\frac{k}{m}\right) \mathbb{1}_{A_{k}}(x), \quad \text { where } A_{k}:=\left(\frac{k-1}{m}, \frac{k}{m}\right]
$$

and $g_{m}(0):=f(0)$. Since $f$ is continuous on a compact interval, it is uniformly continuous on $[0,1]$ and there exists $M>0$ such that $|f(x)|<M$. Hence, for any $\epsilon>0$, there exists an $N>0$ such that for any $m>N$ we have

$$
\left|f(x)-g_{m}(x)\right|<\epsilon, \quad \forall x \in[0,1] .
$$

Hence for any $m>N$ and any $i \in[n]$ we have

$$
\left|\mu_{n, q_{n}}\left(f\left(\frac{\pi(i)}{n}\right)\right)-\mu_{n, q_{n}}\left(g_{m}\left(\frac{\pi(i)}{n}\right)\right)\right|<\epsilon
$$

and

$$
\left|\int_{0}^{1} f(y) \cdot u\left(\frac{i}{n}, y, \beta\right) d y-\int_{0}^{1} g_{m}(y) \cdot u\left(\frac{i}{n}, y, \beta\right) d y\right|<\epsilon .
$$

Moreover, we have

$$
\begin{aligned}
& \left|\mu_{n, q_{n}}\left(g_{m}\left(\frac{\pi(i)}{n}\right)\right)-\int_{0}^{1} g_{m}(y) \cdot u\left(\frac{i}{n}, y, \beta\right) d y\right| \\
\leq & \sum_{k=1}^{m}\left|f\left(\frac{k}{m}\right)\right|\left|\mu_{n, q_{n}}\left(\mathbb{1}_{A_{k}}\left(\frac{\pi(i)}{n}\right)\right)-\int_{0}^{1} \mathbb{1}_{A_{k}}(y) \cdot u\left(\frac{i}{n}, y, \beta\right) d y\right| \\
\leq & \sum_{k=1}^{m} M\left|\mu_{n, q_{n}}\left(\mathbb{1}_{A_{k}}\left(\frac{\pi(i)}{n}\right)\right)-\int_{A_{k}} u\left(\frac{i}{n}, y, \beta\right) d y\right|,
\end{aligned}
$$

Hence by triangle inequality, the first claim (1.4) follows from Lemma 2.1.1, (2.60) and (2.61). To prove the second claim (1.5), we use the same technique by approximating $f$ by simple functions $g_{m}$. Note that by Lemma 2.2.1, for any $m>N$ and any $1 \leq i<$ $j \leq n$, we have

$$
\left|\operatorname{Cov}_{n}\left(f\left(\frac{\pi(i)}{n}\right), f\left(\frac{\pi(j)}{n}\right)\right)-\operatorname{Cov}_{n}\left(g_{m}\left(\frac{\pi(i)}{n}\right), g_{m}\left(\frac{\pi(j)}{n}\right)\right)\right|<4 M \epsilon
$$

Note that

$$
\left|\operatorname{Cov}_{n}\left(g_{m}\left(\frac{\pi(i)}{n}\right), g_{m}\left(\frac{\pi(j)}{n}\right)\right)\right|
$$




$$
\begin{aligned}
& =\left|\sum_{k=1}^{m} \sum_{l=1}^{m} f\left(\frac{k}{m}\right) f\left(\frac{l}{m}\right) \operatorname{Cov}_{n}\left(\mathbb{1}_{A_{k}}\left(\frac{\pi(i)}{n}\right), \mathbb{1}_{A_{l}}\left(\frac{\pi(j)}{n}\right)\right)\right| \\
& \leq M^{2} \sum_{k=1}^{m} \sum_{l=1}^{m}\left|\operatorname{Cov}_{n}\left(\mathbb{1}_{A_{k}}\left(\frac{\pi(i)}{n}\right), \mathbb{1}_{A_{l}}\left(\frac{\pi(j)}{n}\right)\right)\right| .
\end{aligned}
$$

(1.5) follows from Lemma 2.1.2 and (2.62).

\subsection{Convergence of the Empirical Measure}

Recall that, under the conditions in Theorem 2, we need to show the convergence of the empirical measure induced by $\left\{\left(\frac{i}{n}, \frac{\tau \circ \pi(i)}{n}\right)\right\}_{i \in[n]}$. Note that, by relabeling the indices, we have $\left\{\left(\frac{i}{n}, \frac{\tau \circ \pi(i)}{n}\right)\right\}_{i \in[n]}=\left\{\left(\frac{\pi^{-1}(i)}{n}, \frac{\tau(i)}{n}\right)\right\}_{i \in[n]}$. Since $\pi$ and $\tau$ are independent, for a given $i$, the $x$ coordinate and $y$ coordinate of $\left(\frac{\pi^{-1}(i)}{n}, \frac{\tau(i)}{n}\right)$ are independent. We will exploit this property to establish the first and second moment estimates of the number of these points which fall inside a given rectangle.

Recall that, in Section 2.1.1, for any $\pi \in S_{n}$, we define $L_{\pi}$ as the empirical probability measure of $\left\{\left(\frac{i}{n}, \frac{\pi(i)}{n}\right)\right\}_{i \in[n]}$, i.e.,

$$
L_{\pi}(R):=\frac{1}{n} \sum_{i=1}^{n} \mathbb{1}_{R}\left(\frac{i}{n}, \frac{\pi(i)}{n}\right), \quad \forall R \in \mathcal{B}_{[0,1] \times[0,1]} .
$$

Similarly, we now define $L_{\pi, \tau}$ to be the empirical probability measure of $\left\{\left(\frac{\pi(i)}{n}, \frac{\tau(i)}{n}\right)\right\}_{i \in[n]}$. That is

$$
L_{\pi, \tau}(R):=\frac{1}{n} \sum_{i=1}^{n} \mathbb{1}_{R}\left(\frac{\pi(i)}{n}, \frac{\tau(i)}{n}\right), \quad \forall R \in \mathcal{B}_{[0,1] \times[0,1]} .
$$

Lemma 2.1.1 and Lemma 2.1.2 imply the following weak convergence for $L_{\pi, \tau}$.

Lemma 2.3.1. Under the same conditions as Theorem 2, for any $R=\left(x_{1}, x_{2}\right] \times$ $\left(y_{1}, y_{2}\right] \subset[0,1] \times[0,1]$, we have

$$
\lim _{n \rightarrow \infty} \mathbb{P}_{n}\left(\left|L_{\pi, \tau}(R)-\int_{R} \rho(x, y) d x d y\right|>\epsilon\right)=0
$$

for any $\epsilon>0$. Here $\rho(x, y)$ is the density function defined in Theorem 2.

Proof. Let $\bar{R}=\left[x_{1}, x_{2}\right] \times\left[y_{1}, y_{2}\right]$ be the closure of $R$. Since, for any vertical or horizontal line $l$ and any $\pi, \tau \in S_{n}$, we have $L_{\pi, \tau}(l) \leq \frac{1}{n}$, it follows that

$$
\left|L_{\pi, \tau}(R)-L_{\pi, \tau}(\bar{R})\right| \leq \frac{2}{n}
$$


Then, given $\epsilon>0$, for any $n>\frac{4}{\epsilon}$, by triangle inequality and the fact that $\int_{R} \rho(x, y) d x d y=\int_{\bar{R}} \rho(x, y) d x d y$, we get

$$
\begin{aligned}
& \left|L_{\pi, \tau}(R)-\int_{R} \rho(x, y) d x d y\right|>\epsilon \\
\Rightarrow & \left|L_{\pi, \tau}(\bar{R})-\int_{\bar{R}} \rho(x, y) d x d y\right|>\frac{\epsilon}{2} .
\end{aligned}
$$

Hence, it suffices to show (2.63) for $R=\left[x_{1}, x_{2}\right] \times\left[y_{1}, y_{2}\right]$. In the remainder of the proof, let $R:=\left[x_{1}, x_{2}\right] \times\left[y_{1}, y_{2}\right]$. We will show

$$
\begin{aligned}
& \lim _{n \rightarrow \infty} \mathbb{E}_{n}\left(L_{\pi, \tau}(R)\right)=\int_{R} \rho(x, y) d x d y \\
& \lim _{n \rightarrow \infty} \operatorname{Var}_{n}\left(L_{\pi, \tau}(R)\right)=0
\end{aligned}
$$

Then, (2.63) follows from (2.64) and (2.65) by Chebyshev's inequality and triangle inequality.

Let $A=\left[x_{1}, x_{2}\right]$ and $B=\left[y_{1}, y_{2}\right]$. Define

$$
\begin{aligned}
\delta_{n}^{(i)} & :=\mu_{n, q_{n}}\left(\mathbb{1}_{A}\left(\frac{\pi(i)}{n}\right)\right)-\int_{A} u\left(x, \frac{i}{n}, \beta\right) d x, \\
\delta_{n}^{\prime(i)} & :=\mu_{n, q_{n}^{\prime}}\left(\mathbb{1}_{B}\left(\frac{\tau(i)}{n}\right)\right)-\int_{B} u\left(\frac{i}{n}, y, \gamma\right) d y, \\
\delta_{n} & :=\max _{i \in[n]}\left(\left|\delta_{n}^{(i)}\right|\right) \quad \text { and } \quad \delta_{n}^{\prime}:=\max _{i \in[n]}\left(\left|\delta_{n}^{\prime}(i)\right|\right) .
\end{aligned}
$$

Then, by Lemma 2.1.1 and the fact that $u(x, y, \beta)=u(y, x, \beta)$, for any $\epsilon>0$, there exists $N_{1}>0$ such that, for any $n>N_{1}$,

$$
\delta_{n}<\frac{\epsilon}{3} \quad \text { and } \quad \delta_{n}^{\prime}<\frac{\epsilon}{3}
$$

Without loss of generality, assume $0<\epsilon<1$. Then, for any $n>N_{1}$ and any $i \in[n]$, we have

$$
\begin{aligned}
& \left|\mu_{n, q_{n}}\left(\mathbb{1}_{A}\left(\frac{\pi(i)}{n}\right)\right) \mu_{n, q_{n}^{\prime}}\left(\mathbb{1}_{B}\left(\frac{\tau(i)}{n}\right)\right)-\int_{R} u\left(x, \frac{i}{n}, \beta\right) u\left(\frac{i}{n}, y, \gamma\right) d x d y\right| \\
= & \mid\left(\delta_{n}^{(i)}+\int_{A} u\left(x, \frac{i}{n}, \beta\right) d x\right)\left(\delta_{n}^{\prime(i)}+\int_{B} u\left(\frac{i}{n}, y, \gamma\right) d y\right) \\
& -\int_{A} u\left(x, \frac{i}{n}, \beta\right) d x \cdot \int_{B} u\left(\frac{i}{n}, y, \gamma\right) d y \mid \\
\leq & \left|\delta_{n}^{\prime(i)}\right| \int_{A} u\left(x, \frac{i}{n}, \beta\right) d x+\left|\delta_{n}^{(i)}\right| \int_{B} u\left(\frac{i}{n}, y, \gamma\right) d y+\left|\delta_{n}^{(i)} \delta_{n}^{\prime(i)}\right|
\end{aligned}
$$




$$
<\frac{\epsilon}{3}+\frac{\epsilon}{3}+\frac{\epsilon}{3}=\epsilon
$$

Here we use Lemma 2.1.9 in the last inequality. Hence, for any $n>N_{1}$,

$$
\begin{aligned}
& \left|\mathbb{E}_{n}\left(L_{\pi, \tau}(R)\right)-\frac{1}{n} \sum_{i=1}^{n} \int_{R} u\left(x, \frac{i}{n}, \beta\right) u\left(\frac{i}{n}, y, \gamma\right) d x d y\right| \\
= & \left|\frac{1}{n} \sum_{i=1}^{n} \mathbb{E}_{n}\left(\mathbb{1}_{R}\left(\frac{\pi(i)}{n}, \frac{\tau(i)}{n}\right)\right)-\frac{1}{n} \sum_{i=1}^{n} \int_{R} u\left(x, \frac{i}{n}, \beta\right) u\left(\frac{i}{n}, y, \gamma\right) d x d y\right| \\
\leq & \frac{1}{n} \sum_{i=1}^{n}\left|\mathbb{E}_{n}\left(\mathbb{1}_{A}\left(\frac{\pi(i)}{n}\right) \mathbb{1}_{B}\left(\frac{\tau(i)}{n}\right)\right)-\int_{R} u\left(x, \frac{i}{n}, \beta\right) u\left(\frac{i}{n}, y, \gamma\right) d x d y\right| \\
= & \frac{1}{n} \sum_{i=1}^{n}\left|\mu_{n, q_{n}}\left(\mathbb{1}_{A}\left(\frac{\pi(i)}{n}\right)\right) \mu_{n, q_{n}^{\prime}}\left(\mathbb{1}_{B}\left(\frac{\tau(i)}{n}\right)\right)-\int_{R} u\left(x, \frac{i}{n}, \beta\right) u\left(\frac{i}{n}, y, \gamma\right) d x d y\right| \\
< & \epsilon .
\end{aligned}
$$

Here the last equality follows from the fact that $(\pi, \tau) \sim \mu_{n, q_{n}} \times \mu_{n, q_{n}^{\prime}}$ under $\mathbb{P}_{n}$, and the last inequality follows from (2.66).

Since $u(x, y, \beta)$ and $u(x, y, \gamma)$ are bounded on $[0,1] \times[0,1]$, by the definition of Riemann integral and the dominated convergence theorem, we have

$$
\begin{aligned}
& \lim _{n \rightarrow \infty} \frac{1}{n} \sum_{i=1}^{n} \int_{R} u\left(x, \frac{i}{n}, \beta\right) u\left(\frac{i}{n}, y, \gamma\right) d x d y \\
= & \int_{R}\left(\lim _{n \rightarrow \infty} \frac{1}{n} \sum_{i=1}^{n} u\left(x, \frac{i}{n}, \beta\right) u\left(\frac{i}{n}, y, \gamma\right)\right) d x d y \\
= & \int_{R}\left(\int_{0}^{1} u(x, t, \beta) u(t, y, \gamma) d t\right) d x d y \\
= & \int_{R} \rho(x, y) d x d y .
\end{aligned}
$$

Hence, (2.64) follows from (2.67) and (2.68).

To show (2.65), similarly, by Lemma 2.1.2, for any $\epsilon>0$, there exists $N_{2}>0$ such that, for any $n>N_{2}$,

$$
\begin{aligned}
& \max _{\substack{i \neq j \\
i, j \in[n]}}\left|\mu_{n, q_{n}}\left(\mathbb{1}_{A}\left(\frac{\pi(i)}{n}\right) \mathbb{1}_{A}\left(\frac{\pi(j)}{n}\right)\right)-\mu_{n, q_{n}}\left(\mathbb{1}_{A}\left(\frac{\pi(i)}{n}\right)\right) \mu_{n, q_{n}}\left(\mathbb{1}_{A}\left(\frac{\pi(j)}{n}\right)\right)\right|<\frac{\epsilon}{4}, \\
& \max _{\substack{i \neq j \\
i, j \in[n]}}\left|\mu_{n, q_{n}^{\prime}}\left(\mathbb{1}_{B}\left(\frac{\tau(i)}{n}\right) \mathbb{1}_{B}\left(\frac{\tau(j)}{n}\right)\right)-\mu_{n, q_{n}^{\prime}}\left(\mathbb{1}_{B}\left(\frac{\tau(i)}{n}\right)\right) \mu_{n, q_{n}^{\prime}}\left(\mathbb{1}_{B}\left(\frac{\tau(j)}{n}\right)\right)\right|<\frac{\epsilon}{4} .
\end{aligned}
$$

Without loss of generality, assume $0<\epsilon<1$. Then, similar to (2.66), for any $n>N_{2}$ and any $1 \leq i<j \leq n$,

$$
\left|\operatorname{Cov}_{n}\left(\mathbb{1}_{A}\left(\frac{\pi(i)}{n}\right) \mathbb{1}_{B}\left(\frac{\tau(i)}{n}\right), \mathbb{1}_{A}\left(\frac{\pi(j)}{n}\right) \mathbb{1}_{B}\left(\frac{\tau(j)}{n}\right)\right)\right|
$$




$$
\begin{aligned}
& =\mid \mathbb{E}_{n}\left(\mathbb{1}_{A}\left(\frac{\pi(i)}{n}\right) \mathbb{1}_{B}\left(\frac{\tau(i)}{n}\right) \mathbb{1}_{A}\left(\frac{\pi(j)}{n}\right) \mathbb{1}_{B}\left(\frac{\tau(j)}{n}\right)\right) \\
& \quad-\mathbb{E}_{n}\left(\mathbb{1}_{A}\left(\frac{\pi(i)}{n}\right) \mathbb{1}_{B}\left(\frac{\tau(i)}{n}\right)\right) \mathbb{E}_{n}\left(\mathbb{1}_{A}\left(\frac{\pi(j)}{n}\right) \mathbb{1}_{B}\left(\frac{\tau(j)}{n}\right)\right) \mid \\
& =\mid \mu_{n, q_{n}}\left(\mathbb{1}_{A}\left(\frac{\pi(i)}{n}\right) \mathbb{1}_{A}\left(\frac{\pi(j)}{n}\right)\right) \mu_{n, q_{n}^{\prime}}\left(\mathbb{1}_{B}\left(\frac{\tau(i)}{n}\right) \mathbb{1}_{B}\left(\frac{\tau(j)}{n}\right)\right) \\
& \quad-\mu_{n, q_{n}}\left(\mathbb{1}_{A}\left(\frac{\pi(i)}{n}\right)\right) \mu_{n, q_{n}}\left(\mathbb{1}_{A}\left(\frac{\pi(j)}{n}\right)\right) \mu_{n, q_{n}^{\prime}}\left(\mathbb{1}_{B}\left(\frac{\tau(i)}{n}\right)\right) \mu_{n, q_{n}^{\prime}}\left(\mathbb{1}_{B}\left(\frac{\tau(j)}{n}\right)\right) \mid<\frac{\epsilon}{2} .
\end{aligned}
$$

Here the second equality follows from the fact that $(\pi, \tau) \sim \mu_{n, q_{n}} \times \mu_{n, q_{n}^{\prime}}$ under $\mathbb{P}_{n}$, and the last inequality follows by triangle inequality. Specifically, if $0 \leq a_{1}, a_{2}, b_{1}, b_{2} \leq 1$, $\left|a_{1}-a_{2}\right|<\frac{\epsilon}{4}$ and $\left|b_{1}-b_{2}\right|<\frac{\epsilon}{4}$, then we have

$$
\left|a_{1} b_{1}-a_{2} b_{2}\right| \leq\left|a_{1} b_{1}-a_{2} b_{1}\right|+\left|a_{2} b_{1}-a_{2} b_{2}\right| \leq\left|a_{1}-a_{2}\right|+\left|b_{1}-b_{2}\right|<\frac{\epsilon}{2}
$$

Here we choose

$$
\begin{array}{ll}
a_{1}=\mu_{n, q_{n}}\left(\mathbb{1}_{A}\left(\frac{\pi(i)}{n}\right) \mathbb{1}_{A}\left(\frac{\pi(j)}{n}\right)\right), & a_{2}=\mu_{n, q_{n}}\left(\mathbb{1}_{A}\left(\frac{\pi(i)}{n}\right)\right) \mu_{n, q_{n}}\left(\mathbb{1}_{A}\left(\frac{\pi(j)}{n}\right)\right), \\
b_{1}=\mu_{n, q_{n}^{\prime}}\left(\mathbb{1}_{B}\left(\frac{\tau(i)}{n}\right) \mathbb{1}_{B}\left(\frac{\tau(j)}{n}\right)\right), & b_{2}=\mu_{n, q_{n}^{\prime}}\left(\mathbb{1}_{B}\left(\frac{\tau(i)}{n}\right)\right) \mu_{n, q_{n}^{\prime}}\left(\mathbb{1}_{B}\left(\frac{\tau(j)}{n}\right)\right) .
\end{array}
$$

(2.70) follows.

Thus, for any $n>\max \left(N_{2}, \frac{1}{\epsilon}\right)$,

$$
\begin{aligned}
& \operatorname{Var}_{n}\left(L_{\pi, \tau}(R)\right) \\
= & \operatorname{Var}_{n}\left(\frac{1}{n} \sum_{i=1}^{n} \mathbb{1}_{A}\left(\frac{\pi(i)}{n}\right) \mathbb{1}_{B}\left(\frac{\tau(i)}{n}\right)\right) \\
= & \frac{1}{n^{2}} \sum_{i=1}^{n} \operatorname{Var}_{n}\left(\mathbb{1}_{A}\left(\frac{\pi(i)}{n}\right) \mathbb{1}_{B}\left(\frac{\tau(i)}{n}\right)\right) \\
& \quad+\frac{1}{n^{2}} \sum_{\substack{i \neq j \\
i, j \in[n]}} \operatorname{Cov}_{n}\left(\mathbb{1}_{A}\left(\frac{\pi(i)}{n}\right) \mathbb{1}_{B}\left(\frac{\tau(i)}{n}\right), \mathbb{1}_{A}\left(\frac{\pi(j)}{n}\right) \mathbb{1}_{B}\left(\frac{\tau(j)}{n}\right)\right) \\
< & \frac{1}{n^{2}} \cdot \frac{n}{4}+\frac{n(n-1)}{n^{2}} \cdot \frac{\epsilon}{2}<\epsilon .
\end{aligned}
$$

The first inequality follows by (2.69) and the fact that the variance of any indicator function is no greater than $\frac{1}{4}$.

Now we are in the position to prove Theorem 2 
Proof of Theorem 2. First of all, we make the following claim:

Claim: To prove Theorem 2, it suffices to show the case when $f(x, y)=$ $\mathbb{1}_{R}(x, y)$, for any $R=\left(x_{1}, x_{2}\right] \times\left(y_{1}, y_{2}\right] \subset[0,1] \times[0,1]$. This is because for any continuous function $f(x, y)$ and any $\epsilon>0$, we can find a simple function $s(x, y)$ on $(0,1] \times(0,1]$ such that

$$
|f(x, y)-s(x, y)|<\frac{\epsilon}{3} \quad \forall(x, y) \in(0,1] \times(0,1]
$$

where $s(x, y)$ is of the form

$$
s(x, y)=\sum_{j=1}^{m} a_{j} \mathbb{1}_{R_{j}}(x, y),
$$

with $R_{j}=\left(x_{1}^{(j)}, x_{2}^{(j)}\right] \times\left(y_{1}^{(j)}, y_{2}^{(j)}\right] \subset(0,1] \times(0,1]$ and $\left\{R_{j}\right\}_{j=1}^{m}$ is a partition of $(0,1] \times(0,1]$. Hence, we have

$$
\left|\frac{1}{n} \sum_{i=1}^{n} f\left(\frac{i}{n}, \frac{\tau \circ \pi(i)}{n}\right)-\frac{1}{n} \sum_{i=1}^{n} s\left(\frac{i}{n}, \frac{\tau \circ \pi(i)}{n}\right)\right|<\frac{\epsilon}{3},
$$

and

$$
\begin{aligned}
&\left|\int_{0}^{1} \int_{0}^{1} s(x, y) \rho(x, y) d x d y-\int_{0}^{1} \int_{0}^{1} f(x, y) \rho(x, y) d x d y\right| \\
& \leq \int_{0}^{1} \int_{0}^{1}|s(x, y)-f(x, y)| \rho(x, y) d x d y \\
&<\frac{\epsilon}{3}
\end{aligned}
$$

Here we use the fact that, by Lemma 2.1.9,

$$
\int_{0}^{1} \int_{0}^{1} \rho(x, y) d x d y=1
$$

Thus, by (2.71), (2.72) and triangle inequality, we have

$$
\begin{aligned}
& \left|\frac{1}{n} \sum_{i=1}^{n} f\left(\frac{i}{n}, \frac{\tau \circ \pi(i)}{n}\right)-\int_{0}^{1} \int_{0}^{1} f(x, y) \rho(x, y) d x d y\right|>\epsilon \\
\Rightarrow & \left|\frac{1}{n} \sum_{i=1}^{n} s\left(\frac{i}{n}, \frac{\tau \circ \pi(i)}{n}\right)-\int_{0}^{1} \int_{0}^{1} s(x, y) \rho(x, y) d x d y\right|>\frac{\epsilon}{3} .
\end{aligned}
$$

Hence, we get

$$
\mathbb{P}_{n}\left(\left|\frac{1}{n} \sum_{i=1}^{n} f\left(\frac{i}{n}, \frac{\tau \circ \pi(i)}{n}\right)-\int_{0}^{1} \int_{0}^{1} f(x, y) \rho(x, y) d x d y\right|>\epsilon\right)
$$




$$
\begin{aligned}
& \leq \mathbb{P}_{n}\left(\left|\frac{1}{n} \sum_{i=1}^{n} s\left(\frac{i}{n}, \frac{\tau \circ \pi(i)}{n}\right)-\int_{0}^{1} \int_{0}^{1} s(x, y) \rho(x, y) d x d y\right|>\frac{\epsilon}{3}\right) \\
& \leq \sum_{j=1}^{m} \mathbb{P}_{n}\left(\left|\frac{1}{n} \sum_{i=1}^{n} \mathbb{1}_{R_{j}}\left(\frac{i}{n}, \frac{\tau \circ \pi(i)}{n}\right)-\int_{R_{j}} \rho(x, y) d x d y\right|>\frac{\epsilon}{3 m\left|a_{j}\right|}\right)
\end{aligned}
$$

Here the last inequality follows by the union bound. Therefore, to prove Theorem 2, it suffices to show the case when $f(x, y)=\mathbb{1}_{R}(x, y)$, with $R=\left(x_{1}, x_{2}\right] \times\left(y_{1}, y_{2}\right]$. In other words, we need to show that, for any $\epsilon>0$,

$$
\lim _{n \rightarrow \infty} \mathbb{P}_{n}\left(\left|L_{\tau \circ \pi}(R)-\int_{R} \rho(x, y) d x d y\right|>\epsilon\right)=0 .
$$

Here, as defined in (2.1),

$$
L_{\tau \circ \pi}(R):=\frac{1}{n} \sum_{i=1}^{n} \mathbb{1}_{R}\left(\frac{i}{n}, \frac{\tau \circ \pi(i)}{n}\right) .
$$

Then, for any $\pi, \tau \in S_{n}$, we have

$$
\begin{aligned}
\{(i, \tau \circ \pi(i)): i \in[n]\} & =\left\{\left(\pi^{-1}(\pi(i)), \tau(\pi(i))\right): i \in[n]\right\} \\
& =\left\{\left(\pi^{-1}(i), \tau(i)\right): i \in[n]\right\} .
\end{aligned}
$$

The last equality follows since $\{\pi(i)\}_{i \in[n]}=[n]$. Thus, it follows that

$$
L_{\tau \circ \pi}(R)=L_{\pi^{-1}, \tau}(R), \quad \forall R \in \mathcal{B}_{[0,1] \times[0,1]} .
$$

If $(\pi, \tau) \sim \mu_{n, q} \times \mu_{n, q^{\prime}}$, by Lemma 1.1.4, $\left(\pi^{-1}, \tau\right) \sim \mu_{n, q} \times \mu_{n, q^{\prime}}$. Thus, given $(\pi, \tau) \sim \mathbb{P}_{n}$, we have

$$
L_{\tau \circ \pi}(R)=L_{\pi^{-1}, \tau}(R) \stackrel{d}{=} L_{\pi, \tau}(R) .
$$

That is $L_{\tau \circ \pi}(R)$ and $L_{\pi, \tau}(R)$ have the same distribution when $(\pi, \tau) \sim \mathbb{P}_{n}$. Therefore, (2.73) follows by Lemma 2.3.1. 


\section{Chapter 3}

\section{WEAK LAW OF LARGE NUMBERS WHEN AT LEAST ONE PERMUTATION IS CLOSE TO UNIFORMLY RANDOM}

In this chapter we prove Theorem 3 and Theorem 4. In Section 3.1, we argue that the LCS of two permutations is equal to the LIS of a set of points defined by these two permutations. In Section 3.2, we introduce the weak Bruhat order on $S_{n}$ and construct several couplings of Mallows permutations which enable us to establish Lemma 3.3.4 which says that the LIS of those points are close to the LIS of uniformly random points. In Section 3.3, we prove Theorem 3 following the method developed by Deuschel and Zeitouni in [12] for the record lengths of i.i.d. points. In Section 3.4 and Section 3.5, we prove Theorem 4 by constructing couplings of Mallows permutations such that we can bound $\operatorname{LCS}(\pi, \tau)$ by either the LIS or the LCS of the coupled permutations, the limit of which are known. Section 3.6 contains the proofs of two technical lemmas.

\subsection{Reduction LCS problem to LIS problem}

Definition 3.1.1. Given a set of points in $\mathbb{R}^{2}: \boldsymbol{z}=\left\{z_{1}, z_{2}, \ldots, z_{n}\right\}$, where $z_{i}=$ $\left(x_{i}, y_{i}\right) \in \mathbb{R}^{2}$, we say that $\left(z_{i_{1}}, z_{i_{2}}, \ldots, z_{i_{m}}\right)$ is an increasing subsequence if

$$
x_{i_{j}}<x_{i_{j+1}}, \quad y_{i_{j}}<y_{i_{j+1}}, \quad j=1,2, \ldots, m-1 .
$$

Here we do not require $i_{j}<i_{j+1}$. Let LIS( $\left.\boldsymbol{z}\right)$ denote the length of the longest increasing subsequence of $\boldsymbol{z}$.

Definition 3.1.2. Given $\boldsymbol{a}=\left(a_{1}, \ldots, a_{n}\right) \in \mathbb{R}^{n}, \boldsymbol{b}=\left(b_{1}, \ldots, b_{n}\right) \in \mathbb{R}^{n}$, we say that $\left(\left(a_{i_{1}}, b_{i_{1}}\right),\left(a_{i_{2}}, b_{i_{2}}\right), \ldots,\left(a_{i_{m}}, b_{i_{m}}\right)\right)$ is an increasing subsequence between $\boldsymbol{a}$ and $\boldsymbol{b}$ if

$$
a_{i_{j}}<a_{i_{j+1}}, \quad b_{i_{j}}<b_{i_{j+1}}, \quad j=1,2, \ldots, m-1
$$


Here we do not require $i_{j}<i_{j+1}$. Let LIS $(\boldsymbol{a}, \boldsymbol{b})$ denote the length of the longest increasing subsequence between $\boldsymbol{a}$ and $\boldsymbol{b}$. Let $L I S(\boldsymbol{a}):=\operatorname{LIS}(i d, \boldsymbol{a}), \operatorname{LDS}(\boldsymbol{a}):=\operatorname{LIS}\left(i d^{r}, \boldsymbol{a}\right)$. Here $i d=(1,2, \ldots, n)$ denotes the identity in $S_{n}$ and $i d^{r}=(n, \ldots, 2,1)$ denotes the reversal of identity in $S_{n}$. Hence LIS $(\boldsymbol{a})$ is the length of the longest increasing subsequence of $\boldsymbol{a}$ and $\operatorname{LDS}(\boldsymbol{a})$ is the length of the longest decreasing subsequence of $\boldsymbol{a}$.

Note that Definition 3.1.2 allows us to define $\operatorname{LIS}(\pi, \tau)$, the length of the longest increasing subsequence of two permutations, by regarding $\pi$ and $\tau$ as vectors in $\mathbb{R}^{n}$. We show that $\operatorname{LCS}(\pi, \tau)=\operatorname{LIS}\left(\pi^{-1}, \tau^{-1}\right)$, which allows us to reduce the LCS problem to the LIS problem.

Lemma 3.1.3. Given $\pi, \tau \in S_{n}$, we have

$$
L C S(\pi, \tau)=L C S(\sigma \circ \pi, \sigma \circ \tau), \quad L I S(\pi, \tau)=L I S(\pi \circ \sigma, \tau \circ \sigma)
$$

for any $\sigma \in S_{n}$.

Proof. Suppose $\left(a_{1}, a_{2}, \ldots, a_{m}\right)$ is a common subsequence of $\pi$ and $\tau$, then $\left(\sigma\left(a_{1}\right), \ldots, \sigma\left(a_{m}\right)\right)$ is a common subsequence of $\sigma \circ \pi$ and $\sigma \circ \tau$. Hence,

$$
\operatorname{LCS}(\pi, \tau) \leq \operatorname{LCS}(\sigma \circ \pi, \sigma \circ \tau) \leq \operatorname{LCS}\left(\sigma^{-1} \circ \sigma \circ \pi, \sigma^{-1} \circ \sigma \circ \tau\right)=\operatorname{LCS}(\pi, \tau)
$$

Similarly, suppose $\left(\left(\pi\left(i_{1}\right), \tau\left(i_{1}\right)\right),\left(\pi\left(i_{2}\right), \tau\left(i_{2}\right)\right), \ldots,\left(\pi\left(i_{m}\right), \tau\left(i_{m}\right)\right)\right)$ is an increasing subsequence between $\pi$ and $\tau$, then $\left(\left(\pi \circ \sigma\left(i_{1}^{\prime}\right), \tau \circ \sigma\left(i_{1}^{\prime}\right)\right)\right.$, $\left.\left(\pi \circ \sigma\left(i_{2}^{\prime}\right), \tau \circ \sigma\left(i_{2}^{\prime}\right)\right), \ldots,\left(\pi \circ \sigma\left(i_{m}^{\prime}\right), \tau \circ \sigma\left(i_{m}^{\prime}\right)\right)\right)$ is an increasing subsequence between $\pi \circ \sigma$ and $\tau \circ \sigma$, where $i_{k}^{\prime}=\sigma^{-1}\left(i_{k}\right)$ for $k \in[m]$. Hence,

$$
\operatorname{LIS}(\pi, \tau) \leq \operatorname{LIS}(\pi \circ \sigma, \tau \circ \sigma) \leq \operatorname{LIS}\left(\pi \circ \sigma \circ \sigma^{1}, \tau \circ \sigma \circ \sigma^{-1}\right)=\operatorname{LIS}(\pi, \tau) .
$$

Corollary 3.1.4. For any $\pi, \tau \in S_{n}, \operatorname{LCS}(\pi, \tau)=\operatorname{LIS}\left(\pi^{-1}, \tau^{-1}\right)$.

Proof. By the previous lemma, we have

$$
\operatorname{LCS}(\pi, \tau)=\operatorname{LCS}\left(i d, \pi^{-1} \circ \tau\right)=\operatorname{LIS}\left(i d, \pi^{-1} \circ \tau\right)=\operatorname{LIS}\left(\tau^{-1}, \pi^{-1}\right)
$$


In the second equality, we use the following trivial fact,

$$
\operatorname{LCS}(i d, \pi)=\operatorname{LIS}(\pi)=\operatorname{LIS}(i d, \pi)
$$

Here, $i d$ denotes the identity in $S_{n}$, i.e. $i d=(1,2, \ldots, n)$.

\subsection{Weak Bruhat Order}

To prove Lemma 3.3.4, which says that the LIS of the points $\left\{\left(\frac{\pi(i)}{n}, \frac{\tau(i)}{n}\right)\right\}_{i \in[n]}$ that fall in a small box is close to the uniform case, we will establish a coupling of permutations $\left(X, Y, X^{\prime}, X^{\prime \prime}\right)$ such that given $\boldsymbol{a}=\left(a_{1}, \ldots, a_{k}\right)$ with $a_{i} \in[n], \operatorname{LIS}\left(X_{\boldsymbol{a}}, Y_{\boldsymbol{a}}\right)$ can be bounded by $\operatorname{LIS}\left(X_{\boldsymbol{a}}^{\prime}\right)$ and $\operatorname{LDS}\left(X_{\boldsymbol{a}}^{\prime \prime}\right)$. Here $X, X^{\prime}$ and $X^{\prime \prime}$ are distributed according to $\mu_{n, q}$ and $Y$ is independent of $X$ with an arbitrary distribution on $S_{n}$. The main tool we use to construct the coupling is the weak Bruhat order on $S_{n}$.

Recall that for a permutation $\pi \in S_{n}, l(\pi)$ denotes the number of inversions of $\pi$ and $\operatorname{Inv}(\pi)$ denotes the set of inversions of $\pi$. Let $(i, j)$ denote the transposition in $S_{n}$ and $s_{i}:=(i, i+1)$ the adjacent transposition in $S_{n}$.

Definition 3.2.1. The left weak Bruhat order $\left(S_{n}, \leq_{L}\right)$ is defined as the transitive closure of the relations

$$
\pi \leq_{L} \tau \quad \text { if } \quad \tau=s_{i} \circ \pi \text { and } l(\tau)=l(\pi)+1
$$

We are multiplying permutations right-to-left. For instance, $s_{2} \circ 2413=3412$. One characterization of the left weak order is the following (cf. [1]),

$$
\pi \leq_{L} \tau \text { if and only if } \operatorname{Inv}(\pi) \subseteq \operatorname{Inv}(\tau)
$$

The right weak Bruhat order $\left(S_{n}, \leq_{R}\right)$ is defined in the same way except that the covering relationship is given by $\tau=\pi \circ s_{i}$.

Definition 3.2.2. The right weak Bruhat order $\left(S_{n}, \leq_{R}\right)$ is defined as the transitive closure of the relations

$$
\pi \leq_{R} \tau \quad \text { if } \quad \tau=\pi \circ s_{i} \text { and } l(\tau)=l(\pi)+1
$$


From the above definitions, the following proposition follows trivially.

Proposition 3.2.3. For any $\pi, \tau \in S_{n}, \pi \leq_{R} \tau$ if and only if $\pi^{-1} \leq_{L} \tau^{-1}$.

Definition 3.2.4. Let $(\Omega, \leq)$ be a partially ordered set. A non-empty subset $A \subset \Omega$ is called increasing if

$$
\omega \in A \text { and } \omega \leq \omega^{\prime} \Rightarrow \omega^{\prime} \in A
$$

Given two probability measures $\mu_{1}, \mu_{2}$ on $(\Omega, \mathcal{F})$, we say that $\mu_{1}$ is stochastically smaller than $\mu_{2}$, denoted by $\mu_{1} \preceq \mu_{2}$, if

$$
\mu_{1}(A) \leq \mu_{2}(A) \text { for all increasing events } A \text {. }
$$

Lemma 3.2.5. Given the poset $\left(S_{n}, \leq_{L}\right)$, for any $0<q<q^{\prime}$, we have $\mu_{n, q} \preceq \mu_{n, q^{\prime}}$.

Proof. We are going to construct a coupling of two Markov chains $\left(X_{t}, Y_{t}\right)$, such that

1. Both $\left\{X_{t}\right\}$ and $\left\{Y_{t}\right\}$ are irreducible, aperiodic Markov chains on $S_{n}$.

2. The stationary distributions for $\left\{X_{t}\right\}$ and $\left\{Y_{t}\right\}$ are $\mu_{n, q}$ and $\mu_{n, q^{\prime}}$ respectively.

3. $X_{t} \leq_{L} Y_{t}$ for any $t \geq 0$.

By 3, for any increasing subset $A \subseteq S_{n}$, we have

$$
\mathbb{P}\left(X_{t} \in A\right)=\mathbb{P}\left(X_{t} \in A, X_{t} \leq_{L} Y_{t}\right) \leq \mathbb{P}\left(Y_{t} \in A\right)
$$

Also, by properties 1 and 2, we have

$$
\mu_{n, q}(A)=\lim _{t \rightarrow \infty} \mathbb{P}\left(X_{t} \in A\right), \quad \text { and } \quad \mu_{n, q^{\prime}}(A)=\lim _{t \rightarrow \infty} \mathbb{P}\left(Y_{t} \in A\right)
$$

Combining (3.1) and (3.2), we get

$$
\mu_{n, q}(A) \leq \mu_{n, q^{\prime}}(A), \quad \text { for all increasing subsets } A
$$

The remainder of the proof is devoted to the construction of the coupling $\left(X_{t}, Y_{t}\right)$ which satisfies the three properties above. The coupling $\left(X_{t}, Y_{t}\right)$ is defined as follows, 
- When time $t=0, X_{0}=Y_{0}=i d$. That is, both chains start from the identity in $S_{n}$.

- At each time $t$, sample three independent random variables: $U, F, B$. $U$ samples the integers from 1 to $n-1$ uniformly. $F$ and $B$ are two coins with the probability of heads being $\frac{1}{1+q^{\prime}}$ and $\frac{\left(1+q^{\prime}\right) q}{(1+q) q^{\prime}}$ respectively.

Suppose $U=i$. Then flip the coins $F$ and $B$ and update the chains according to the following rules:

Case 1 if $X^{-1}(i)<X^{-1}(i+1)$ and $Y^{-1}(i)<Y^{-1}(i+1)$, then

\begin{tabular}{lll}
\hline$F$ is head & $X_{t+1}=X_{t}$, & $Y_{t+1}=Y_{t}$ \\
\hline$F$ is tail, $B$ is head & $X_{t+1}=s_{i} \circ X_{t}$, & $Y_{t+1}=s_{i} \circ Y_{t}$ \\
\hline$F$ is tail, $B$ is tail & $X_{t+1}=X_{t}$, & $Y_{t+1}=s_{i} \circ Y_{t}$ \\
\hline
\end{tabular}

Case 2 if $X^{-1}(i)<X^{-1}(i+1)$ and $Y^{-1}(i)>Y^{-1}(i+1)$, then

\begin{tabular}{lll}
\hline$F$ is head & $X_{t+1}=X_{t}$, & $Y_{t+1}=s_{i} \circ Y_{t}$ \\
\hline$F$ is tail, $B$ is head & $X_{t+1}=s_{i} \circ X_{t}$, & $Y_{t+1}=Y_{t}$ \\
\hline$F$ is tail, $B$ is tail & $X_{t+1}=X_{t}$, & $Y_{t+1}=Y_{t}$ \\
\hline
\end{tabular}

Case 3 if $X^{-1}(i)>X^{-1}(i+1)$ and $Y^{-1}(i)>Y^{-1}(i+1)$, then

\begin{tabular}{lll}
\hline$F$ is head & $X_{t+1}=s_{i} \circ X_{t}$, & $Y_{t+1}=s_{i} \circ Y_{t}$ \\
\hline$F$ is tail, $B$ is head & $X_{t+1}=X_{t}$, & $Y_{t+1}=Y_{t}$ \\
\hline$F$ is tail, $B$ is tail & $X_{t+1}=s_{i} \circ X_{t}$, & $Y_{t+1}=Y_{t}$ \\
\hline
\end{tabular}

By the definition above and the following facts, it is easy to check that the three properties listed at the beginning of the proof are satisfied. 
- The adjacent transpositions $\left\{s_{i}\right\}$ generate $S_{n}$ under the group multiplication in $S_{n}$.

- If $\pi^{-1}(i)=j, \pi^{-1}(i+1)=k$ and $j<k$, we have $\operatorname{Inv}\left(s_{i} \circ \pi\right)=\operatorname{Inv}(\pi) \cup\{(j, k)\}$.

- If $\pi^{-1}(i)=j, \pi^{-1}(i+1)=k$ and $j>k$, we have $\operatorname{Inv}\left(s_{i} \circ \pi\right)=\operatorname{Inv}(\pi) \backslash\{(k, j)\}$.

- $\pi \leq_{L} \tau$ if and only if $\operatorname{Inv}(\pi) \subseteq \operatorname{Inv}(\tau)$.

- Both chains $X_{t}, Y_{t}$ satisfy the detailed balance equations, i.e. that the Mallows distribution satisfies that

$$
\mu_{n, q}(\pi) \cdot \mathbb{P}\left(X_{t+1}=\tau \mid X_{t}=\pi\right)=\mu_{n, q}(\tau) \cdot \mathbb{P}\left(X_{t+1}=\pi \mid X_{t}=\tau\right)
$$

for any $\pi, \tau \in S_{n}$ and similarly for the chain $Y_{t}$.

The stochastic dominance between $\mu_{n, q}$ and $\mu_{n, q^{\prime}}$ also holds if we change the underlying partial order to the right weak Bruhat order.

Lemma 3.2.6. Given the poset $\left(S_{n}, \leq_{R}\right)$, for any $0<q<q^{\prime}$, we have $\mu_{n, q} \preceq \mu_{n, q^{\prime}}$.

Proof. Given any increasing set $A$ in $\left(S_{n}, \leq_{R}\right)$, let $A^{-1}:=\left\{\pi^{-1}: \pi \in A\right\}$. By Proposition 3.2.3, $A^{-1}$ is an increasing set in $\left(S_{n}, \leq_{L}\right)$. Since $l(\pi)=l\left(\pi^{-1}\right)$, we have $\mu_{n, q}(\pi)=\mu_{n, q}\left(\pi^{-1}\right)$, whence $\mu_{n, q}(A)=\mu_{n, q}\left(A^{-1}\right)$. Then, by Lemma 3.2.5 and Definition 3.2.4, we have

$$
\mu_{n, q}(A)=\mu_{n, q}\left(A^{-1}\right) \leq \mu_{n, q^{\prime}}\left(A^{-1}\right)=\mu_{n, q^{\prime}}(A)
$$

Definition 3.2.7. Given $\pi \in S_{n}$ and $\boldsymbol{a}=\left(a_{1}, a_{2}, \ldots, a_{k}\right)$, where $a_{i} \in[n]$ and $a_{1}<$ $a_{2}<\cdots<a_{k}$, let $\pi(\boldsymbol{a})=\left(\pi\left(a_{1}\right), \pi\left(a_{2}\right), \ldots, \pi\left(a_{k}\right)\right)$. Let $\pi_{\boldsymbol{a}} \in S_{k}$ denote the permutation induced by $\pi(\boldsymbol{a})$, i. e. $\pi_{\boldsymbol{a}}(i)=j$ if $\pi\left(a_{i}\right)$ is the $j$-th smallest term in $\pi(\boldsymbol{a})$. 
Corollary 3.2.8. Let $n \in \mathbb{N}$, and $\boldsymbol{a}=\left(a_{1}, a_{2}, \ldots, a_{k}\right)$, where $a_{i} \in[n]$ and $a_{1}<a_{2}<$ $\cdots<a_{k}$.

(a) For any $q \geq 1$, we can construct a pair of random variables $(U, V)$ such that $U$ is uniformly distributed on $S_{k}, V$ has the same distribution as $\pi_{\boldsymbol{a}}$, where $\pi \sim \mu_{n, q}$, and $U \leq_{L} V$.

(b) For any $q \leq 1$, we can construct a pair of random variables $(U, V)$ such that $U$ is uniformly distributed on $S_{k}, V$ has the same distribution as $\pi_{\boldsymbol{a}}$, where $\pi \sim \mu_{n, q}$, and $V \leq_{L} U$.

Proof. Here we only prove part (a). Part (b) follows by a similar argument. Since $q \geq 1$, by Lemma 3.2.5 and Strassen's theorem [27], there exist two random variables $(X, Y)$ defined on the same probability space such that $X$ is the uniform measure on $S_{n}, Y \sim \mu_{n, q}$ and $X \leq_{L} Y$. Then we can show $X_{\boldsymbol{a}} \leq_{L} Y_{\boldsymbol{a}}$. Since $\pi \leq_{L} \tau$ if and only if $\operatorname{Inv}(\pi) \subseteq \operatorname{Inv}(\tau)$, we have

$$
\begin{aligned}
\operatorname{Inv}\left(X_{\boldsymbol{a}}\right) & =\left\{(i, j): 1 \leq i<j \leq k \text { and } X\left(a_{i}\right)>X\left(a_{j}\right)\right\} \\
& \subset\left\{(i, j): 1 \leq i<j \leq k \text { and } Y\left(a_{i}\right)>Y\left(a_{j}\right)\right\}=\operatorname{Inv}\left(Y_{\boldsymbol{a}}\right) .
\end{aligned}
$$

Hence, if we define $U:=X_{\boldsymbol{a}}$ and $V:=Y_{\boldsymbol{a}}$, part (a) follows by the fact that $X_{\boldsymbol{a}}$ is uniformly distributed on $S_{k}$.

Lemma 3.2.9. Given $\pi, \tau \in S_{k}$ with $\pi \leq_{L} \tau$, for any $n \geq k, 0<q \leq 1$ and $a_{1}<\cdots<$ $a_{k}$ with $a_{i} \in[n]$, there exists a coupling $(X, Y)$ such that $X \sim \mu_{n, q}, Y \sim \mu_{n, q}$ and

$$
\operatorname{LIS}\left(X_{\boldsymbol{a}}, \pi\right) \geq \operatorname{LIS}\left(Y_{\boldsymbol{a}}, \tau\right)
$$

Here $\boldsymbol{a}=\left(a_{1}, a_{2}, \ldots, a_{k}\right)$.

Proof. First, we claim that it suffices to show the case when $\tau$ covers $\pi$ in $\left(S_{k}, \leq_{L}\right)$, that is $l(\tau)=l(\pi)+1$ and $\tau=s_{i} \circ \pi$ for some $i \in[k-1]$. The claim can be shown by induction on the Kendall's tau distance of $\pi$ and $\tau$, i.e., the minimum number of adjacent transpositions multiplied to $\pi$ from the left to get $\tau$. Suppose we have 
$\pi \leq_{L} \sigma \leq_{L} \tau$ in $S_{k}$ with $l(\pi)<l(\sigma)<l(\tau)$. By the induction hypothesis there exist two couplings $(X, Y)$ and $\left(Y^{\prime}, Z\right)$, which are not necessarily defined in the same probability space, such that $X, Y, Y^{\prime}, Z$ have the same marginal distribution $\mu_{n, q}$ and

$$
\operatorname{LIS}\left(X_{\boldsymbol{a}}, \pi\right) \geq \operatorname{LIS}\left(Y_{\boldsymbol{a}}, \sigma\right), \quad \operatorname{LIS}\left(Y_{\boldsymbol{a}}^{\prime}, \sigma\right) \geq \operatorname{LIS}\left(Z_{\boldsymbol{a}}, \tau\right)
$$

We can construct a new coupling $\left(X^{\prime}, Z^{\prime}\right)$ as follows,

(1) Sample a permutation $\xi \in S_{n}$ according to the distribution $\mu_{n, q}$.

(2) Sample $X^{\prime}$ according to the induced distribution on $S_{n}$ by the first coupling $(X, Y)$ conditioned on $Y=\xi$.

(3) Sample $Z^{\prime}$ according to the induced distribution on $S_{n}$ by the second coupling $\left(Y^{\prime}, Z\right)$ conditioned on $Y^{\prime}=\xi$.

By the law of total probability, it is easily seen that $X^{\prime} \sim \mu_{n, q}$ and $Z^{\prime} \sim \mu_{n, q}$. Also, regardless of which permutation $\xi$ being sampled in the first step, by (3.3), we have

$$
\operatorname{LIS}\left(X_{\boldsymbol{a}}^{\prime}, \pi\right) \geq \operatorname{LIS}\left(\xi_{\boldsymbol{a}}, \sigma\right) \geq \operatorname{LIS}\left(Z_{\boldsymbol{a}}^{\prime}, \tau\right)
$$

In the remainder of the proof, we assume $\tau=s_{i} \circ \pi$ and $l(\tau)=l(\pi)+1$. Note that, for any $\sigma \in S_{n}$,

$$
\sigma \circ(i, j)=(\sigma(i), \sigma(j)) \circ \sigma, \quad \sigma_{\boldsymbol{a}} \circ(i, j)=\left(\sigma \circ\left(a_{i}, a_{j}\right)\right)_{\boldsymbol{a}}
$$

Let $r=a_{\pi^{-1}(i)}$ and $t=a_{\pi^{-1}(i+1)}$. Since $l(\tau)=l(\pi)+1$, we have $\pi^{-1}(i)<\pi^{-1}(i+1)$, thus, $r<t$. Let $A:=\left\{\{\sigma, \sigma \circ(r, t)\}: \sigma \in S_{n}\right.$ and $\left.\sigma(r)<\sigma(t)\right\}$. Clearly, $A$ is a partition of $S_{n}$. Then we construct the coupling $(X, Y)$ as follows:

(1) Choose a set in $A$ according to measure $\mu_{n, q}$, i. e. the set $\{\sigma, \sigma \circ(r, t)\}$ is chosen with probability $\mu_{n, q}(\{\sigma, \sigma \circ(r, t)\})$.

(2) Suppose the set $\{\sigma, \sigma \circ(r, t)\}$, with $\sigma(r)<\sigma(t)$, is chosen in the first step. Flip a coin with probability of heads being

$$
p=\frac{q^{l(\sigma)}-q^{l(\sigma \circ(r, t))}}{q^{l(\sigma)}+q^{l(\sigma \circ(r, t))}} .
$$


(3) If the outcome is head, then we set $X=Y=\sigma$.

(4) If the outcome is tail, then, with equal probability, we set either $X=\sigma, Y=$ $\sigma \circ(r, t)$ or $X=\sigma \circ(r, t), Y=\sigma$.

Here, in the second step, the probability of heads $p$ is nonnegative because we have $0<q \leq 1$ and the following fact:

$$
i<j \text { and } \sigma(i)<\sigma(j) \Rightarrow l(\sigma)<l(\sigma \circ(i, j)), \quad \forall \sigma \in S_{n}
$$

It can be verified that $(X, Y)$ thus defined has the correct marginal distribution $\mu_{n, q}$. In the following we show that

$$
\operatorname{LIS}\left(X_{\boldsymbol{a}} \circ \pi^{-1}\right) \geq \operatorname{LIS}\left(Y_{\boldsymbol{a}} \circ \tau^{-1}\right)
$$

Then, the lemma follows by Lemma 3.1 .3 because, let $i d$ denote the identity in $S_{k}$, we have

$$
\begin{aligned}
& \operatorname{LIS}\left(X_{\boldsymbol{a}} \circ \pi^{-1}\right)=\operatorname{LIS}\left(X_{\boldsymbol{a}} \circ \pi^{-1}, i d\right)=\operatorname{LIS}\left(X_{\boldsymbol{a}}, \pi\right), \\
& \operatorname{LIS}\left(Y_{\boldsymbol{a}} \circ \tau^{-1}\right)=\operatorname{LIS}\left(Y_{\boldsymbol{a}} \circ \tau^{-1}, i d\right)=\operatorname{LIS}\left(Y_{\boldsymbol{a}}, \tau\right) .
\end{aligned}
$$

Suppose the set $\{\sigma, \sigma \circ(r, t)\}$, with $\sigma(r)<\sigma(t)$, is chosen in the first step. If the outcome in the second step is tail, we verify that $X_{\boldsymbol{a}} \circ \pi^{-1}=Y_{\boldsymbol{a}} \circ \tau^{-1}$. When $X=\sigma$, $Y=\sigma \circ(r, t)$, by $(3.4)$, we have

$$
\begin{aligned}
X_{\boldsymbol{a}} \circ \pi^{-1} & =\sigma_{\boldsymbol{a}} \circ \pi^{-1}, \\
Y_{\boldsymbol{a}} \circ \tau^{-1} & =(\sigma \circ(r, t))_{\boldsymbol{a}} \circ \pi^{-1} \circ s_{i} \\
& =(\sigma \circ(r, t))_{\boldsymbol{a}} \circ\left(\pi^{-1}(i), \pi^{-1}(i+1)\right) \circ \pi^{-1} \\
& =(\sigma \circ(r, t) \circ(r, t))_{\boldsymbol{a}} \circ \pi^{-1} \\
& =\sigma_{\boldsymbol{a}} \circ \pi^{-1} .
\end{aligned}
$$

When $X=\sigma \circ(r, t), Y=\sigma$, again by (3.4), we have

$$
X_{\boldsymbol{a}} \circ \pi^{-1}=(\sigma \circ(r, t))_{\boldsymbol{a}} \circ \pi^{-1}
$$




$$
\begin{aligned}
& =\sigma_{\boldsymbol{a}} \circ\left(\pi^{-1}(i), \pi^{-1}(i+1)\right) \circ \pi^{-1} \\
& =\sigma_{\boldsymbol{a}} \circ \pi^{-1} \circ s_{i}, \\
Y_{\boldsymbol{a}} \circ \tau^{-1} & =\sigma_{\boldsymbol{a}} \circ \pi^{-1} \circ s_{i} .
\end{aligned}
$$

If the outcome in the second step is head, we have

$$
X_{\boldsymbol{a}} \circ \pi^{-1}=\sigma_{\boldsymbol{a}} \circ \pi^{-1} \quad \text { and } \quad Y_{\boldsymbol{a}} \circ \tau^{-1}=\sigma_{\boldsymbol{a}} \circ \pi^{-1} \circ s_{i}
$$

Since $\sigma(r)<\sigma(t)$, i. e., $\sigma\left(a_{\pi^{-1}(i)}\right)<\sigma\left(a_{\pi^{-1}(i+1)}\right)$, we have $\sigma_{\boldsymbol{a}} \circ \pi^{-1}(i)<\sigma_{\boldsymbol{a}} \circ \pi^{-1}(i+1)$. Hence $Y_{\boldsymbol{a}} \circ \tau^{-1}$ covers $X_{\boldsymbol{a}} \circ \pi^{-1}$ in $\left(S_{k}, \leq_{R}\right)$. (3.5) follows.

Remark. A special case of Lemma 3.2.9 is when $k=n$, in which the only choice for $\boldsymbol{a}$ is the vector $(1,2,3, \ldots, n)$ whence $X_{\boldsymbol{a}}=X, Y_{\boldsymbol{a}}=Y$.

We can prove a similar result for the case when $q \geq 1$.

Lemma 3.2.10. Given $\pi, \tau \in S_{k}$ with $\pi \leq_{L} \tau$, for any $n \geq k, q \geq 1$ and $a_{1}<\cdots<a_{k}$ with $a_{i} \in[n]$, there exists a coupling $(X, Y)$ such that $X \sim \mu_{n, q}, Y \sim \mu_{n, q}$ and

$$
\operatorname{LIS}\left(X_{\boldsymbol{a}}, \pi\right) \leq \operatorname{LIS}\left(Y_{\boldsymbol{a}}, \tau\right)
$$

Here $\boldsymbol{a}=\left(a_{1}, a_{2}, \ldots, a_{k}\right)$.

Proof. Given $\pi \in S_{n}$, recall that $\pi^{r}$ denote the reversal of $\pi$. For any $\pi \in S_{n}$, we have $\operatorname{Inv}\left(\pi^{r}\right)=\{(i, j): 1 \leq i<j \leq n$ and $(n+1-j, n+1-i) \notin \operatorname{Inv}(\pi)\}$. Hence, $\pi \leq_{L} \tau$ implies $\pi^{r} \geq_{L} \tau^{r}$. By Lemma 3.2.9, there exists a coupling $(U, V)$ such that $U \sim \mu_{n, 1 / q}$, $V \sim \mu_{n, 1 / q}$ and

$$
\operatorname{LIS}\left(U_{\boldsymbol{a}^{\prime}}, \pi^{r}\right) \leq \operatorname{LIS}\left(V_{\boldsymbol{a}^{\prime}}, \tau^{r}\right) .
$$

Here $\boldsymbol{a}^{\prime}=\left(a_{1}^{\prime}, a_{2}^{\prime}, \ldots, a_{k}^{\prime}\right)$ with $a_{i}^{\prime}=n+1-a_{k+1-i}$.

Define $(X, Y):=\left(U^{r}, V^{r}\right)$. By Lemma 1.1.4, $X \sim \mu_{n, q}, Y \sim \mu_{n, q}$. Moreover, we have

$$
\begin{aligned}
\operatorname{LIS}\left(X_{\boldsymbol{a}}, \pi\right) & =\operatorname{LIS}\left(\left(X_{\boldsymbol{a}}\right)^{r}, \pi^{r}\right)=\operatorname{LIS}\left(\left(X^{r}\right)_{\boldsymbol{a}^{\prime}}, \pi^{r}\right)=\operatorname{LIS}\left(U_{\boldsymbol{a}^{\prime}}, \pi^{r}\right) \\
& \leq \operatorname{LIS}\left(V_{\boldsymbol{a}^{\prime}}, \tau^{r}\right)=\operatorname{LIS}\left(\left(Y^{r}\right)_{\boldsymbol{a}^{\prime}}, \tau^{r}\right)=\operatorname{LIS}\left(\left(Y_{\boldsymbol{a}}\right)^{r}, \tau^{r}\right) \\
& =\operatorname{LIS}\left(Y_{\boldsymbol{a}}, \tau\right) .
\end{aligned}
$$


Lemma 3.2.11. Given $\boldsymbol{a}=\left(a_{1}, a_{2}, \ldots, a_{k}\right)$, where $a_{1}<\cdots<a_{k}$ and $a_{i} \in[n]$, for any $0<q \leq 1$ and any distribution $\nu$ on $S_{k}$, there exists a coupling $(X, Y, Z)$ such that the following holds,

(a) $X$ and $Y$ are independent.

(b) $X \sim \mu_{n, q}, Y \sim \nu$ and $Z \sim \mu_{n, q}$.

(c) $\operatorname{LIS}\left(X_{\boldsymbol{a}}, Y\right) \leq \operatorname{LIS}\left(Z_{\boldsymbol{a}}\right)$.

Proof. Let $i d_{k}$ denote the identity in $S_{k}$. By the definition of weak bruhat order, for any $\xi \in S_{k}$, we have $i d_{k} \leq_{L} \xi$. Hence, given $\xi \in S_{k}$, by Lemma 3.2.9, there exists a coupling $(U, V)$ such that $U \sim \mu_{n, q}, V \sim \mu_{n, q}$ and $\operatorname{LIS}\left(U_{\boldsymbol{a}}, \xi\right) \leq \operatorname{LIS}\left(V_{\boldsymbol{a}}, i d_{k}\right)=\operatorname{LIS}\left(V_{\boldsymbol{a}}\right)$. Then we construct the coupling $(X, Y, Z)$ as follows

- Sample $Y$ according to the distribution $\nu$.

- Conditioned on $Y=\xi,(X, Z)$ has the same distribution as $(U, V)$ defined above.

First, we point out that $X$ and $Y$ are independent. Since whatever value $Y$ takes, the conditional distribution of $X$ is $\mu_{n, q}$. Moreover, it can be seen that $X, Y$ and $Z$ have the right marginal distribution. Finally, (c) holds by the construction of the coupling.

We can prove a similar result for the case when $q \geq 1$.

Lemma 3.2.12. Given $\boldsymbol{a}=\left(a_{1}, a_{2}, \ldots, a_{k}\right)$, where $a_{1}<\cdots<a_{k}$ and $a_{i} \in[n]$, for any $q \geq 1$ and any distribution $\nu$ on $S_{k}$, there exists a coupling $(X, Y, Z)$ such that the following holds,

(a) $X$ and $Y$ are independent.

(b) $X \sim \mu_{n, q}, Y \sim \nu$ and $Z \sim \mu_{n, q}$.

(c) $\operatorname{LIS}\left(X_{\boldsymbol{a}}, Y\right) \geq \operatorname{LIS}\left(Z_{\boldsymbol{a}}\right)$. 
Proof. Let $i d_{k}$ denote the identity in $S_{k}$. By the definition of weak bruhat order, for any $\xi \in S_{k}$, we have $i d_{k} \leq_{L} \xi$. Hence, given $\xi \in S_{k}$, by Lemma 3.2.10, there exists a coupling $(U, V)$ such that $U \sim \mu_{n, q}, V \sim \mu_{n, q}$ and $\operatorname{LIS}\left(U_{\boldsymbol{a}}, \xi\right) \geq \operatorname{LIS}\left(V_{\boldsymbol{a}}, i d_{k}\right)=\operatorname{LIS}\left(V_{\boldsymbol{a}}\right)$. Then we construct the coupling $(X, Y, Z)$ as follows

- Sample $Y$ according to the distribution $\nu$.

- Conditioned on $Y=\xi,(X, Z)$ has the same distribution as $(U, V)$ defined above.

First, we point out that $X$ and $Y$ are independent. Since whatever value $Y$ takes, the conditional distribution of $X$ is $\mu_{n, q}$. Moreover, it can be seen that $X, Y$ and $Z$ have the right marginal distribution. Finally, (c) holds by the construction of the coupling.

Lemma 3.2.13. Given $\boldsymbol{a}=\left(a_{1}, a_{2}, \ldots, a_{k}\right)$, where $a_{1}<\cdots<a_{k}$ and $a_{i} \in[n]$. Define $\overline{\boldsymbol{a}}:=\left\{n+1-a_{k}, n+1-a_{k-1}, \ldots, n+1-a_{1}\right\}$. For any $0<q \leq 1$ and any distribution $\nu$ on $S_{k}$, there exists a coupling $(X, Y, Z)$ such that the following holds,

(a) $X$ and $Y$ are independent.

(b) $X \sim \mu_{n, q}, Y \sim \nu$ and $Z \sim \mu_{n, 1 / q}$.

(c) $\operatorname{LIS}\left(X_{\boldsymbol{a}}, Y\right) \geq \operatorname{LIS}\left(Z_{\overline{\boldsymbol{a}}}\right)$.

Proof. Recall that $\pi^{r}$ denotes the reversal of $\pi$. If $\pi \sim \nu$, we use $\nu^{r}$ to denote the distribution of $\pi^{r}$. Clearly, $\nu=\left(\nu^{r}\right)^{r}$. By Lemma 3.2.12, there exists a coupling $(U, V, Z)$ such that

- $U$ and $V$ are independent.

- $U \sim \mu_{n, 1 / q}, V \sim \nu^{r}$ and $Z \sim \mu_{n, 1 / q}$.

- $\operatorname{LIS}\left(U_{\overline{\boldsymbol{a}}}, V\right) \geq \operatorname{LIS}\left(Z_{\overline{\boldsymbol{a}}}\right)$.

Define $X:=U^{r}$ and $Y:=V^{r}$. We have

$$
\operatorname{LIS}\left(U_{\overline{\boldsymbol{a}}}, V\right)=\operatorname{LIS}\left(\left\{\left(U_{\overline{\boldsymbol{a}}}(i), V(i)\right)\right\}_{i \in[k]}\right)
$$




$$
\begin{aligned}
& =\operatorname{LIS}\left(\left\{\left(\left(U_{\overline{\boldsymbol{a}}}\right)^{r}(i), V^{r}(i)\right)\right\}_{i \in[k]}\right) \\
& =\operatorname{LIS}\left(\left\{\left(\left(U^{r}\right)_{\boldsymbol{a}}(i), V^{r}(i)\right)\right\}_{i \in[k]}\right) \\
& =\operatorname{LIS}\left(\left\{\left(X_{\boldsymbol{a}}(i), Y(i)\right)\right\}_{i \in[k]}\right) \\
& =\operatorname{LIS}\left(X_{\boldsymbol{a}}, Y\right),
\end{aligned}
$$

The lemma follows.

\subsection{Proof of WLLN when both $\beta$ and $\gamma$ are finite}

We start this section by introducing the following two lemmas which can be seen as generalizations of Corollary 4.3 in [22]. That result shows that the LIS of a Mallows permutation scaled by $n^{-1 / 2}$ can be bounded within a multiplicative interval of $e^{|\beta|}$ around 2. We postpone the proofs of these two lemmas to Section 3.6. For any positive integer $n$ and $m \in[n]$, define

$$
Q(n, m):=\left\{\left(b_{1}, b_{2}, \ldots, b_{m}\right): b_{i} \in[n] \text { and } b_{i}<b_{i+1} \text { for all } i\right\} .
$$

Lemma 3.3.1. Suppose that $\left\{q_{n}\right\}_{n=1}^{\infty}$ is a sequence such that $q_{n} \geq 1$ and $\liminf _{n \rightarrow \infty} n\left(1-q_{n}\right)=\beta$, with $\beta \in \mathbb{R}$. For any sequence $\left\{k_{n}\right\}_{n=1}^{\infty}$ such that $k_{n} \in[n]$ and $\lim _{n \rightarrow \infty} k_{n}=\infty$, we have

$$
\lim _{n \rightarrow \infty} \max _{\boldsymbol{b} \in Q\left(n, k_{n}\right)} \mu_{n, q_{n}}\left(\pi \in S_{n}: \frac{\operatorname{LIS}\left(\pi_{\boldsymbol{b}}\right)}{\sqrt{k_{n}}} \notin\left(2 e^{\frac{\beta}{2}}-\epsilon, 2+\epsilon\right)\right)=0,
$$

for any $\epsilon>0$.

Lemma 3.3.2. Suppose that $\left\{q_{n}\right\}_{n=1}^{\infty}$ is a sequence such that $0<q_{n} \leq 1$ and $\limsup _{n \rightarrow \infty} n\left(1-q_{n}\right)=\beta<\ln 2$. For any sequence $\left\{k_{n}\right\}_{n=1}^{\infty}$ such that $k_{n} \in[n]$ and $\lim _{n \rightarrow \infty} k_{n}=\infty$, we have

$$
\lim _{n \rightarrow \infty} \max _{\boldsymbol{b} \in Q\left(n, k_{n}\right)} \mu_{n, q_{n}}\left(\pi \in S_{n}: \frac{\operatorname{LIS}\left(\pi_{\boldsymbol{b}}\right)}{\sqrt{k_{n}}} \notin\left(2-\epsilon, 2 e^{\frac{\beta}{2}}+\epsilon\right)\right)=0,
$$

for any $\epsilon>0$. 
Next, we introduce the following way to sample a permutation according to $\mu_{n, q}$ which will be used in the proofs. Given $\boldsymbol{c}=\left\{c_{1}, c_{2}, \ldots, c_{m}\right\}$, where $c_{i} \in \mathbb{Z}^{+}$and $\sum_{i=1}^{m} c_{i}=n$, define

$$
\begin{aligned}
& d_{0}:=0, \quad d_{k}:=\sum_{i=1}^{k} c_{i} \quad \forall k \in[m], \\
& A(\boldsymbol{c}):=\left\{\left(A_{1}, A_{2}, \ldots, A_{m}\right):\left\{A_{i}\right\}_{i \in[m]} \text { is a partition of }[n],\left|A_{i}\right|=c_{i}\right\} .
\end{aligned}
$$

Given $\left(A_{1}, \ldots, A_{m}\right) \in A(\boldsymbol{c})$, define the inversion number of $\left(A_{1}, \ldots, A_{m}\right)$ as follows,

$$
\begin{aligned}
& l\left(\left(A_{1}, \ldots, A_{m}\right)\right):= \\
& \mid\left\{(x, y): x>y \text { and there exists } i<j \text { such that } x \in A_{i}, y \in A_{j}\right\} \mid .
\end{aligned}
$$

Let $\boldsymbol{a}_{i}$ be the vector which consists of the numbers in $A_{i}$ in increasing order. There exists a bijection $f_{\boldsymbol{c}}$ between $S_{n}$ and $A(\boldsymbol{c}) \times S_{c_{1}} \times S_{c_{2}} \times \cdots \times S_{c_{m}}$ such that, for any $\pi \in S_{n}, f_{\boldsymbol{c}}(\pi)=\left(\left(A_{1}, A_{2}, \ldots, A_{m}\right), \tau_{1}, \tau_{2}, \ldots, \tau_{m}\right)$ if and only if

$$
\left\{\pi(j): j \in A_{i}\right\}=\left\{d_{i-1}+1, d_{i-1}+2, \ldots, d_{i}\right\}, \quad \pi_{\boldsymbol{a}_{i}}=\tau_{i}, \quad \forall i \in[m] .
$$

From the definition above, it is not hard to see that the following relation holds,

$$
l(\pi)=l\left(\left(A_{1}, A_{2}, \ldots, A_{m}\right)\right)+\sum_{i=1}^{m} l\left(\tau_{i}\right) .
$$

Define the random variable $X_{\boldsymbol{c}}$ which takes value in $A(\boldsymbol{c})$ such that

$$
\mathbb{P}\left(X_{\boldsymbol{c}}=\left(A_{1}, A_{2}, \ldots, A_{m}\right)\right) \propto q^{l\left(\left(A_{1}, A_{2}, \ldots, A_{m}\right)\right)} .
$$

Independent of $X_{\boldsymbol{c}}$, let $Y_{1}, Y_{2}, \ldots, Y_{m}$ be independent random variables such that, for any $i \in[m], Y_{i} \sim \mu_{c_{i}, q}$. Define $Z:=f_{\boldsymbol{c}}^{-1}\left(X_{\boldsymbol{c}}, Y_{1}, Y_{2}, \ldots, Y_{m}\right)$. By (3.6), we have $Z \sim \mu_{n, q}$, since

$$
\mathbb{P}(Z=\pi) \propto q^{l(\pi)} .
$$

As our last step in preparation for the proof of Lemma 3.3.4, we introduce the following elementary result in analysis. 
Lemma 3.3.3. Suppose $\left\{B_{i}\right\}_{i=1}^{\infty}$ is a partition of $\mathbb{N}$, i.e. $\cup_{i=1}^{\infty} B_{i}=\mathbb{N}$ and $B_{i} \cap B_{j}=$ $\varnothing, \forall i \neq j$. Moreover, each $B_{i}$ is a finite nonempty set. Given a sequence $\left\{x_{i}\right\}_{i=1}^{\infty}$, if $\lim _{n \rightarrow \infty} x_{b_{n}}=a$, for any sequence $\left\{b_{i}\right\}_{i=1}^{\infty}$ with $b_{i} \in B_{i}$, then we have $\lim _{n \rightarrow \infty} x_{n}=a$.

Proof. We prove the lemma by contradiction. Suppose $\lim _{n \rightarrow \infty} x_{n}=a$ does not hold. Then there exists $\epsilon>0$ and a subsequence $\left\{x_{n_{j}}\right\}_{j=1}^{\infty}$ such that $x_{n_{j}} \notin(a-\epsilon, a+\epsilon)$ for all $j$. Since each $B_{i}$ is a finite set, without loss of generality, we may assume that each $B_{i}$ contains at most one $n_{j}$. Then, we can construct a sequence $\left\{b_{i}\right\}_{i=1}^{\infty}$ with $b_{i} \in B_{i}$, such that $x_{b_{i}} \notin(a-\epsilon, a+\epsilon)$ infinitely often. Specifically, we define the sequence $\left\{b_{i}\right\}_{i=1}^{\infty}$ as follows. For each $i$, if there exists an $n_{j} \in B_{i}$, let $b_{i}=n_{j}$, otherwise, let $b_{i}$ be an arbitrary number in $B_{i}$. Thus, we get the contradiction.

For any $\pi, \tau \in S_{n}$, define $\boldsymbol{z}(\pi, \tau):=\left\{\left(\frac{\pi(i)}{n}, \frac{\tau(i)}{n}\right)\right\}_{i \in[n]}$. Let $l_{R}(\pi, \tau)$ denote the length of the longest increasing subsequence of $\boldsymbol{z}(\pi, \tau)$ within $R$. The following lemma addresses the size of the LIS of $\boldsymbol{z}(\pi, \tau)$ in a small rectangle and this result will be the most crucial building block used to show both the upper and lower bounds in Theorem 3.

Lemma 3.3.4. Let $R=\left(x_{1}, x_{2}\right] \times\left(y_{1}, y_{2}\right] \subset[0,1] \times[0,1]$. Under the same conditions as in Lemma 2.3.1, if $\Delta x|\beta|<\ln 2$, we have

$$
\lim _{n \rightarrow \infty} \mathbb{P}_{n}\left(\frac{l_{R}(\pi, \tau)}{\sqrt{n \rho(R)}} \in\left(2 e^{-\Delta x|\beta| / 2}-\epsilon, 2 e^{\Delta x|\beta| / 2}+\epsilon\right)\right)=1
$$

for any $\epsilon>0$, where $\rho(R):=\iint_{R} \rho(x, y) d x d y$ and $\Delta x:=x_{2}-x_{1}$.

Proof. To simplify the proof, we divide the lemma into the following three cases:

Case $1: \beta>0$ or $\beta=0$ and $q_{n} \leq 1$ when $n$ is sufficiently large.

Case 2 : $\beta<0$ or $\beta=0$ and $q_{n} \geq 1$ when $n$ is sufficiently large.

Case $3: \beta=0$. 
Firstly, Case 3 follows from Case 1 and Case 2 because if $\lim _{n \rightarrow \infty} n\left(1-q_{n}\right)=0$, we can divide the sequence $\left\{q_{n}\right\}_{n=1}^{\infty}$ into two disjoint subsequences such that one of them falls into Case 1 and the other falls into Case 2.

Next we argue that Case 2 follows from Case 1. If $\pi \sim \mu_{n, q}$, by Lemma 1.1.4, we have $\pi^{r} \sim \mu_{n, 1 / q}$. Trivially, for any $\pi, \tau \in S_{n}$, we have $\boldsymbol{z}(\pi, \tau)=\boldsymbol{z}\left(\pi^{r}, \tau^{r}\right)$. Since $\lim _{n \rightarrow \infty} n\left(1-q_{n}\right)=\beta \in \mathbb{R}$, we have $\lim _{n \rightarrow \infty} q_{n}=1$. Hence,

$$
\lim _{n \rightarrow \infty} n\left(1-1 / q_{n}\right)=\lim _{n \rightarrow \infty} n\left(q_{n}-1\right) / q_{n}=-\beta
$$

Therefore, Case 2 follows from Case 1 by considering the reversal of $\pi$ and $\tau$ in (3.7). Specifically, if $\pi \sim \mu_{n, q_{n}}$ and $\tau \sim \mu_{n, q_{n}^{\prime}}$, after reversing, we have $\pi^{r} \sim \mu_{n, 1 / q_{n}}$ and $\tau^{r} \sim \mu_{n, 1 / q_{n}^{\prime}}$ and the $n$ points induced by $\pi$ and $\tau$ do not change, i.e., $\boldsymbol{z}(\pi, \tau)=\boldsymbol{z}\left(\pi^{r}, \tau^{r}\right)$. To prove Case 1 , in the following, we assume $x_{1}, y_{1}>0$ and $x_{2}, y_{2}<1$. The proofs for the cases when $x_{1}=0$ or $y_{1}=0$ or $x_{2}=1$ or $y_{2}=1$ are similar.

Let $x_{3}=y_{3}=1$. Given $n \in \mathbb{N}$, we will sample $(\pi, \tau)$ according to $\mathbb{P}_{n}$ by the method introduced before Lemma 3.3.3. Define

$$
\begin{array}{lll}
d_{n, i}:=\left\lfloor n x_{i}\right\rfloor, & c_{n, i}:=d_{n, i}-d_{n, i-1}, & \text { for } i=1,2,3, \\
d_{n, i}^{\prime}:=\left\lfloor n y_{i}\right\rfloor, & c_{n, i}^{\prime}:=d_{n, i}^{\prime}-d_{n, i-1}^{\prime}, & \text { for } i=1,2,3 .
\end{array}
$$

Here we assume that $d_{n, 0}=d_{n, 0}^{\prime}=0$. Then, it is trivial that

$$
\begin{array}{ll}
d_{n, i}=\left|\left\{j \in[n]: \frac{j}{n} \in\left(0, x_{i}\right]\right\}\right|, & c_{n, 2}=\left|\left\{j \in[n]: \frac{j}{n} \in\left(x_{1}, x_{2}\right]\right\}\right|, \\
d_{n, i}^{\prime}=\left|\left\{j \in[n]: \frac{j}{n} \in\left(0, y_{i}\right]\right\}\right|, & c_{n, 2}^{\prime}=\left|\left\{j \in[n]: \frac{j}{n} \in\left(y_{1}, y_{2}\right]\right\}\right| .
\end{array}
$$

Since $\lim _{n \rightarrow \infty} \frac{\lfloor n x\rfloor}{n}=x, \forall x \in \mathbb{R}$, it follows that $\lim _{n \rightarrow \infty} \frac{d_{n, i}}{n}=x_{i}$. Hence

$$
\lim _{n \rightarrow \infty} \frac{c_{n, 2}}{n}=x_{2}-x_{1}=\Delta x
$$

Next, for any nonnegative integer $i$, define $B_{i}:=\left\{n \in \mathbb{N}: c_{n, 2}=i\right\}$. Clearly, $\left\{B_{i}\right\}_{i=0}^{\infty}$ thus defined is a partition of $\mathbb{N}$ and we show that each $B_{i}$ is a nonempty finite set. Since, by (3.8), $\lim _{n \rightarrow \infty} c_{n, 2}=\infty$, we conclude that each $B_{i}$ is a finite set. From the definition of $d_{n, i}$, it is easily seen that the sequence $\left\{d_{n, 1}\right\}$ is nondecreasing and the 
increment of consecutive terms is either 0 or 1 . The same is true for the sequence $\left\{d_{n, 2}\right\}$. Hence, we have

$$
\left|c_{n+1,2}-c_{n, 2}\right|=\left|d_{n+1,2}-d_{n, 2}-\left(d_{n+1,1},-d_{n, 1}\right)\right| \leq 1
$$

Since $c_{1,2} \in B_{0}$ and $\lim _{n \rightarrow \infty} c_{n, 2}=\infty$, the inequality above guarantees that each $B_{i}$ is nonempty.

Next, define $\boldsymbol{c}_{n}=\left(c_{n, 1}, c_{n, 2}, c_{n, 3}\right)$ and $\boldsymbol{c}_{n}^{\prime}=\left(c_{n, 1}^{\prime}, c_{n, 2}^{\prime}, c_{n, 3}^{\prime}\right)$. Define $X_{\boldsymbol{c}_{n}}$ which takes values in $A\left(\boldsymbol{c}_{n}\right)$ such that

$$
\mathbb{P}\left(X_{\boldsymbol{c}_{n}}=\left(A_{1}, A_{2}, A_{3}\right)\right) \propto q_{n}^{l\left(\left(A_{1}, A_{2}, A_{3}\right)\right)}, \quad \forall\left(A_{1}, A_{2}, A_{3}\right) \in A\left(\boldsymbol{c}_{n}\right) .
$$

Independently, define three independent random variables $Y_{n, 1}, Y_{n, 2}, Y_{n, 3}$ such that $Y_{n, i} \sim \mu_{c_{n, i}, q_{n}}$. Independent of all the variables defined above, define $X_{\boldsymbol{c}_{n}^{\prime}}$ and $Y_{n, 1}^{\prime}$, $Y_{n, 2}^{\prime}, Y_{n, 3}^{\prime}$ in the same fashion. That is, $X_{\boldsymbol{c}_{n}^{\prime}}$ takes value in $A\left(\boldsymbol{c}_{n}^{\prime}\right)$ with

$$
\mathbb{P}\left(X_{\boldsymbol{c}_{n}^{\prime}}=\left(A_{1}^{\prime}, A_{2}^{\prime}, A_{3}^{\prime}\right)\right) \propto\left(q_{n}^{\prime}\right)^{l\left(\left(A_{1}^{\prime}, A_{2}^{\prime}, A_{3}^{\prime}\right)\right)}, \quad \forall\left(A_{1}^{\prime}, A_{2}^{\prime}, A_{3}^{\prime}\right) \in A\left(\boldsymbol{c}_{n}^{\prime}\right)
$$

and $Y_{n, 1}^{\prime}, Y_{n, 2}^{\prime}, Y_{n, 3}^{\prime}$ are three independent random variables with $Y_{n, i}^{\prime} \sim \mu_{c_{n, i}^{\prime}, q_{n}^{\prime}}$. Define

$$
\pi:=f_{\boldsymbol{c}_{n}}^{-1}\left(X_{\boldsymbol{c}_{n}}, Y_{n, 1}, Y_{n, 2}, Y_{n, 3}\right), \quad \tau:=f_{\boldsymbol{c}^{\prime}{ }_{n}}^{-1}\left(X_{\boldsymbol{c}_{n}^{\prime}}, Y_{n, 1}^{\prime}, Y_{n, 2}^{\prime}, Y_{n, 3}^{\prime}\right)
$$

From the discussion before Lemma 3.3.3, it follows that $(\pi, \tau)$ thus defined has distribution $\mathbb{P}_{n}$. Moreover, given $X_{\boldsymbol{c}_{n}}=\left(A_{1}, A_{2}, A_{3}\right)$ and $X_{\boldsymbol{c}_{n}^{\prime}}=\left(A_{1}^{\prime}, A_{2}^{\prime}, A_{3}^{\prime}\right)$, we have

$$
A_{2}=\left\{i \in[n]: \frac{\pi(i)}{n} \in\left(x_{1}, x_{2}\right]\right\}, \quad A_{2}^{\prime}=\left\{i \in[n]: \frac{\tau(i)}{n} \in\left(y_{1}, y_{2}\right]\right\} .
$$

Hence, we have

$$
A_{2} \cap A_{2}^{\prime}=\left\{i \in[n]:\left(\frac{\pi(i)}{n}, \frac{\tau(i)}{n}\right) \in R\right\} .
$$

Define $M=|\boldsymbol{z}(\pi, \tau) \cap R|$, i. e. $M$ denotes the number of points $\left\{\left(\frac{\pi(i)}{n}, \frac{\tau(i)}{n}\right)\right\}_{i=1}^{n}$ within $R$. Then, by (3.9), we have $M=\left|A_{2} \cap A_{2}^{\prime}\right|$. Hence, $M$ only depends on the values of $X_{\boldsymbol{c}_{n}}$ and $X_{\boldsymbol{c}_{n}^{\prime}}$ and is independent of $\cup_{i \in[3]}\left\{Y_{n, i}, Y_{n, i}^{\prime}\right\}$. Next, we point out that, conditioning on $X_{\boldsymbol{c}_{n}}=\left(A_{1}, A_{2}, A_{3}\right)$ and $X_{\boldsymbol{c}_{n}^{\prime}}=\left(A_{1}^{\prime}, A_{2}^{\prime}, A_{3}^{\prime}\right), l_{R}(\pi, \tau)$ is determined by $Y_{n, 2}$ and $Y_{n, 2}^{\prime}$. To see this, we first define a new function $I$ as follows, given any finite 
set $A \subset \mathbb{Z}$ and any $a \in A$, define $I(A, a):=k$ if $a$ is the $k$-th smallest number in $A$. Suppose $A_{2} \cap A_{2}^{\prime}=\left\{a_{j}\right\}_{j \in[M]}$ with $a_{1}<a_{2}<\cdots<a_{M}$. Define $\boldsymbol{b} \in Q\left(c_{n, 2}, M\right)$ and $\boldsymbol{b}^{\prime} \in Q\left(c_{n, 2}^{\prime}, M\right)$ by

$$
\begin{aligned}
\boldsymbol{b} & :=\left(I\left(A_{2}, a_{1}\right), I\left(A_{2}, a_{2}\right), \ldots, I\left(A_{2}, a_{M}\right)\right), \\
\boldsymbol{b}^{\prime} & :=\left(I\left(A_{2}^{\prime}, a_{1}\right), I\left(A_{2}^{\prime}, a_{2}\right), \ldots, I\left(A_{2}^{\prime}, a_{M}\right)\right) .
\end{aligned}
$$

Note that $\boldsymbol{b}$ and $\boldsymbol{b}^{\prime}$ are determined by $A_{2}$ and $A_{2}^{\prime}$. Then, we have

$$
l_{R}(\pi, \tau)=\operatorname{LIS}\left(\left(Y_{n, 2}\right)_{\boldsymbol{b}},\left(Y_{n, 2}^{\prime}\right)_{\boldsymbol{b}^{\prime}}\right) .
$$

Because, conditioning on $X_{\boldsymbol{c}_{n}}=\left(A_{1}, A_{2}, A_{3}\right)$, we know that $\left\{\pi(i): i \in A_{2}\right\}=\left\{d_{n, 1}+\right.$ $\left.1, d_{n, 1}+2, \ldots, d_{n, 2}\right\}$. The value of $Y_{n, 2}$ determines the relative ordering of $\pi(i)$ for those $i \in A_{2}$. Similarly, the value of $Y_{n, 2}^{\prime}$ determines the relative ordering of $\tau(i)$ for those $i \in A_{2}^{\prime}$.

Now we are in the position to prove (3.7) for Case 1. From the discussion above and Lemma 3.3.3, it suffices to show that, for any sequence $\left\{s_{n}\right\}_{n=1}^{\infty}$ with $s_{n} \in B_{n}$, i.e., when $c_{s_{n}, 2}=n$, we have

$$
\lim _{n \rightarrow \infty} \mathbb{P}_{s_{n}}\left(\frac{l_{R}(\pi, \tau)}{\sqrt{s_{n} \rho(R)}} \in\left(2 e^{-\Delta x \beta / 2}-\epsilon, 2 e^{\Delta x \beta / 2}+\epsilon\right)\right)=1,
$$

for any $\epsilon>0$. Note that by the definition of $\mathbb{P}_{s_{n}}$ in Lemma 2.3.1, $\pi$ and $\tau$ above are of size $s_{n}$ with $\pi \sim \mu_{s_{n}, q_{s_{n}}}, \tau \sim \mu_{s_{n}, q_{s_{n}}^{\prime}}$.

We separate the proof of (3.12) into two parts. Specifically, we need to show that

$$
\lim _{n \rightarrow \infty} \mathbb{P}_{s_{n}}\left(\frac{l_{R}(\pi, \tau)}{\sqrt{s_{n} \rho(R)}}<2 e^{\Delta x \beta / 2}+\epsilon\right)=1
$$

and

$$
\lim _{n \rightarrow \infty} \mathbb{P}_{s_{n}}\left(\frac{l_{R}(\pi, \tau)}{\sqrt{s_{n} \rho(R)}}>2 e^{-\Delta x \beta / 2}-\epsilon\right)=1,
$$

for any $\epsilon>0$.

Since $\left\{s_{n}\right\}_{n \geq 1}$ is a subsequence of $\{i\}_{i \geq 0}, \lim _{n \rightarrow \infty} s_{n}=\infty$. Hence, by (3.8) and the fact that $c_{s_{n}, 2}=n$, we get

$$
\lim _{n \rightarrow \infty} \frac{n}{s_{n}}=\lim _{n \rightarrow \infty} \frac{c_{s_{n}, 2}}{s_{n}}=\Delta x
$$


Thus,

$$
\lim _{n \rightarrow \infty} n\left(1-q_{s_{n}}\right)=\lim _{n \rightarrow \infty} \frac{n}{s_{n}} s_{n}\left(1-q_{s_{n}}\right)=\Delta x \beta<\ln 2 .
$$

To prove (3.13), for any $\epsilon>0$, we can choose $\epsilon_{1}>0$ sufficiently small such that

$$
\left(1-\epsilon_{1}\right)\left(2 e^{\Delta x \beta / 2}+\epsilon\right)>2 e^{\Delta x \beta / 2} .
$$

For this fixed $\epsilon_{1}$, we can choose $\delta>0$ such that

$$
\sqrt{\frac{\rho(R)}{\rho(R)+\delta}}>1-\epsilon_{1} .
$$

Given $n \in \mathbb{N}$, define $k_{n}=\left\lfloor s_{n}(\rho(R)+\delta)\right\rfloor$. Clearly, we have $\lim _{n \rightarrow \infty} k_{n}=\infty$. Moreover, under the conditions of Case 1, $q_{n} \leq 1$ for sufficiently large $n$. Hence, by Lemma 3.3.2, (3.15) and (3.16), there exists $N_{1}>0$ such that, for any $n>N_{1}$, we have

$$
\min _{\boldsymbol{b} \in Q\left(n, k_{n}\right)} \mu_{n, q_{s_{n}}}\left(\eta \in S_{n}: \frac{\operatorname{LIS}\left(\eta_{b}\right)}{\sqrt{k_{n}}}<\left(1-\epsilon_{1}\right)\left(2 e^{\Delta x \beta / 2}+\epsilon\right)\right)>1-\epsilon .
$$

Given $\boldsymbol{b} \in Q\left(n, k_{n}\right)$, for any $\boldsymbol{b}^{\prime}$ which is a subsequence of $\boldsymbol{b}$, we have $\operatorname{LIS}\left(\eta_{\boldsymbol{b}}\right) \geq \operatorname{LIS}\left(\eta_{\boldsymbol{b}^{\prime}}\right)$. Thus we can make (3.18) stronger as follows,

$$
\min _{\boldsymbol{b} \in \bar{Q}\left(n, k_{n}\right)} \mu_{n, q_{s_{n}}}\left(\eta \in S_{n}: \frac{\operatorname{LIS}\left(\eta_{b}\right)}{\sqrt{k_{n}}}<\left(1-\epsilon_{1}\right)\left(2 e^{\Delta x \beta / 2}+\epsilon\right)\right)>1-\epsilon,
$$

where $\bar{Q}\left(n, k_{n}\right)=\cup_{i \in\left[k_{n}\right]} Q(n, i)$. Since $\lim _{n \rightarrow \infty} s_{n}=\infty$, we have

$$
\lim _{n \rightarrow \infty} s_{n}\left(1-q_{s_{n}}\right)=\beta \quad \text { and } \quad \lim _{n \rightarrow \infty} s_{n}\left(1-q_{s_{n}}^{\prime}\right)=\gamma
$$

Hence, by Lemma 2.3.1, there exists $N_{2}>0$ such that, for any $n>N_{2}$, we have

$$
\mathbb{P}_{s_{n}}\left(\frac{|\boldsymbol{z}(\pi, \tau) \cap R|}{s_{n}} \leq \rho(R)+\delta\right)>1-\epsilon .
$$

In the following, let $E_{n}\left(A_{2}, A_{2}^{\prime}\right)$ denote the event that the second entries of $X_{\boldsymbol{c}_{s_{n}}}$ and $X_{\boldsymbol{c}_{s_{n}}^{\prime}}$ are $A_{2}$ and $A_{2}^{\prime}$ respectively. Then, for any $n>\max \left(N_{1}, N_{2}\right)$, we have

$$
\begin{aligned}
& \mathbb{P}_{s_{n}}\left(\frac{l_{R}(\pi, \tau)}{\sqrt{s_{n} \rho(R)}}<2 e^{\Delta x \beta / 2}+\epsilon\right) \\
\geq & \sum_{\left|A_{2} \cap A_{2}^{\prime}\right| \leq k_{n}} \mathbb{P}\left(\frac{l_{R}(\pi, \tau)}{\sqrt{s_{n} \rho(R)}}<2 e^{\Delta x \beta / 2}+\epsilon \mid E_{n}\left(A_{2}, A_{2}^{\prime}\right)\right) \times \mathbb{P}\left(E_{n}\left(A_{2}, A_{2}^{\prime}\right)\right)
\end{aligned}
$$




$$
\begin{aligned}
& =\sum_{\left|A_{2} \cap A_{2}^{\prime}\right| \leq k_{n}} \mathbb{P}\left(\frac{\operatorname{LIS}\left(\left(Y_{s_{n}, 2}\right)_{\boldsymbol{b}},\left(Y_{s_{n}, 2}^{\prime}\right)_{\boldsymbol{b}^{\prime}}\right)}{\sqrt{s_{n} \rho(R)}}<2 e^{\Delta x \beta / 2}+\epsilon \mid E_{n}\left(A_{2}, A_{2}^{\prime}\right)\right) \\
& \times \mathbb{P}\left(E_{n}\left(A_{2}, A_{2}^{\prime}\right)\right) \\
& =\sum_{\left|A_{2} \cap A_{2}^{\prime}\right| \leq k_{n}} \mathbb{P}\left(\frac{\operatorname{LIS}\left(\left(Y_{s_{n}, 2}\right)_{\boldsymbol{b}},\left(Y_{s_{n}, 2}^{\prime}\right)_{\boldsymbol{b}^{\prime}}\right)}{\sqrt{s_{n} \rho(R)}}<2 e^{\Delta x \beta / 2}+\epsilon\right) \times \mathbb{P}\left(E_{n}\left(A_{2}, A_{2}^{\prime}\right)\right) \\
& \geq \sum_{\left|A_{2} \cap A_{2}^{\prime}\right| \leq k_{n}} \mu_{n, q_{s_{n}}}\left(\frac{\operatorname{LIS}\left(\eta_{b}\right)}{\sqrt{s_{n} \rho(R)}}<2 e^{\Delta x \beta / 2}+\epsilon\right) \times \mathbb{P}\left(E_{n}\left(A_{2}, A_{2}^{\prime}\right)\right) \\
& =\sum_{\left|A_{2} \cap A_{2}^{\prime}\right| \leq k_{n}} \mu_{n, q_{s_{n}}}\left(\frac{\operatorname{LIS}\left(\eta_{\boldsymbol{b}}\right)}{\sqrt{s_{n}(\rho(R)+\delta)}}<\frac{\sqrt{\rho(R)}}{\sqrt{\rho(R)+\delta}}\left(2 e^{\Delta x \beta / 2}+\epsilon\right)\right) \\
& \times \mathbb{P}\left(E_{n}\left(A_{2}, A_{2}^{\prime}\right)\right) \\
& \geq \sum_{\left|A_{2} \cap A_{2}^{\prime}\right| \leq k_{n}} \mu_{n, q_{s_{n}}}\left(\frac{\operatorname{LIS}\left(\eta_{b}\right)}{\sqrt{k_{n}}}<\left(1-\epsilon_{1}\right)\left(2 e^{\Delta x \beta / 2}+\epsilon\right)\right) \times \mathbb{P}\left(E_{n}\left(A_{2}, A_{2}^{\prime}\right)\right) \\
& \geq(1-\epsilon) \times \sum_{\left|A_{2} \cap A_{2}^{\prime}\right| \leq k_{n}} \mathbb{P}\left(E_{n}\left(A_{2}, A_{2}^{\prime}\right)\right) \\
& =(1-\epsilon) \times \mathbb{P}_{s_{n}}\left(|\boldsymbol{z}(\pi, \tau) \cap R| \leq k_{n}\right) \\
& =(1-\epsilon) \times \mathbb{P}_{s_{n}}\left(|\boldsymbol{z}(\pi, \tau) \cap R| \leq s_{n}(\rho(R)+\delta)\right) \\
& >(1-\epsilon)^{2} \text {. }
\end{aligned}
$$

Here $\mathbb{P}$ denotes the probability space on which $\left(X_{\boldsymbol{c}_{s_{n}}}, Y_{s_{n}, 1}, Y_{s_{n}, 2}, Y_{s_{n}, 3}\right)$ and $\left(X_{\boldsymbol{c}_{s_{n}}^{\prime}}, Y_{s_{n}, 1}^{\prime}, Y_{s_{n}, 2}^{\prime}, Y_{s_{n}, 3}^{\prime}\right)$ are defined. The first equality follows by (3.11). The second equality follows by independence of $\left(X_{\boldsymbol{c}_{s_{n}}}, X_{\boldsymbol{c}_{s_{n}}^{\prime}}\right)$ and $\left(Y_{s_{n}, 2}, Y_{s_{n}, 2}^{\prime}\right)$. Note that $\boldsymbol{b}$ and $\boldsymbol{b}^{\prime}$ are determined by $A_{2}$ and $A_{2}^{\prime}$ as in (3.10). The second inequality follows by Lemma 3.2.11, since $Y_{s_{n}, 2}$ and $Y_{s_{n}, 2}^{\prime}$ are independent with $Y_{s_{n}, 2} \sim \mu_{n, q_{s_{n}}}$. The third inequality follows by (3.17) and the fact that $k_{n}=\left\lfloor s_{n}(\rho(R)+\delta)\right\rfloor \leq s_{n}(\rho(R)+\delta)$. The fourth inequality follows by (3.19) and the fact that the dimension of $\boldsymbol{b}$ equals to $\left|A_{2} \cap A_{2}^{\prime}\right|$. The last inequality follows by (3.21). Hence, (3.13) follows.

The proof of (3.14) is analogous to the proof of (3.13). First, by (3.15) and the fact that $\lim _{n \rightarrow \infty} q_{n}=1$, we have

$$
\lim _{n \rightarrow \infty} n\left(1-1 / q_{s_{n}}\right)=\lim _{n \rightarrow \infty} \frac{n\left(q_{s_{n}}-1\right)}{q_{s_{n}}}=-\Delta x \beta .
$$


For any $\epsilon>0$, we can choose $\epsilon_{1}>0$ sufficiently small such that

$$
\left(1+\epsilon_{1}\right)\left(2 e^{-\Delta x \beta / 2}-\epsilon\right)<2 e^{-\Delta x \beta / 2} .
$$

For this fixed $\epsilon_{1}$, we can choose $\delta>0$ such that

$$
\sqrt{\frac{\rho(R)}{\rho(R)-\delta}}<1+\epsilon_{1}
$$

Given $n \in \mathbb{N}$, define $k_{n}^{\prime}=\left\lceil s_{n}(\rho(R)-\delta)\right\rceil$. Clearly, we have $\lim _{n \rightarrow \infty} k_{n}^{\prime}=\infty$. Moreover, under conditions of Case $1,1 / q_{n} \geq 1$ for sufficiently large $n$. Hence, by Lemma 3.3.1, (3.22) and (3.23), there exist $N_{3}>0$ such that, for any $n>N_{3}$, we have

$$
\min _{\boldsymbol{b} \in Q\left(n, k_{n}^{\prime}\right)} \mu_{n, 1 / q_{s_{n}}}\left(\eta \in S_{n}: \frac{\operatorname{LIS}\left(\eta_{b}\right)}{\sqrt{k_{n}^{\prime}}}>\left(1+\epsilon_{1}\right)\left(2 e^{-\Delta x \beta / 2}-\epsilon\right)\right)>1-\epsilon .
$$

Given $\boldsymbol{b} \in Q\left(n, k_{n}^{\prime}\right)$, for any $\boldsymbol{b}^{\prime}$ such that $\boldsymbol{b}$ is a subsequence of $\boldsymbol{b}^{\prime}$, we have $\operatorname{LIS}\left(\eta_{\boldsymbol{b}}\right) \leq$ $\operatorname{LIS}\left(\eta_{\boldsymbol{b}^{\prime}}\right)$. Thus we can make (3.25) stronger as follows,

$$
\min _{\boldsymbol{b} \in \hat{Q}\left(n, k_{n}^{\prime}\right)} \mu_{n, 1 / q_{s_{n}}}\left(\eta \in S_{n}: \frac{\operatorname{LIS}\left(\eta_{b}\right)}{\sqrt{k_{n}^{\prime}}}>\left(1+\epsilon_{1}\right)\left(2 e^{-\Delta x \beta / 2}-\epsilon\right)\right)>1-\epsilon,
$$

where $\hat{Q}\left(n, k_{n}^{\prime}\right)=\cup_{k_{n}^{\prime} \leq i \leq n} Q(n, i)$.

By (3.20) and Lemma 2.3.1, there exists $N_{4}>0$ such that, for any $n>N_{4}$, we have

$$
\mathbb{P}_{s_{n}}\left(\frac{|\boldsymbol{z}(\pi, \tau) \cap R|}{s_{n}} \geq \rho(R)-\delta\right)>1-\epsilon
$$

Again, let $E_{n}\left(A_{2}, A_{2}^{\prime}\right)$ denote the event that the second entries of $X_{\boldsymbol{c}_{s_{n}}}$ and $X_{\boldsymbol{c}_{s_{n}}^{\prime}}$ are $A_{2}$ and $A_{2}^{\prime}$ respectively. Then, for any $n>\max \left(N_{3}, N_{4}\right)$, we have

$$
\begin{aligned}
& \mathbb{P}_{s_{n}}\left(\frac{l_{R}(\pi, \tau)}{\sqrt{s_{n} \rho(R)}}>2 e^{-\Delta x \beta / 2}-\epsilon\right) \\
\geq & \sum_{\left|A_{2} \cap A_{2}^{\prime}\right| \geq k_{n}} \mathbb{P}\left(\frac{l_{R}(\pi, \tau)}{\sqrt{s_{n} \rho(R)}}>2 e^{-\Delta x \beta / 2}-\epsilon \mid E_{n}\left(A_{2}, A_{2}^{\prime}\right)\right) \times \mathbb{P}\left(E_{n}\left(A_{2}, A_{2}^{\prime}\right)\right) \\
= & \sum_{\left|A_{2} \cap A_{2}^{\prime}\right| \geq k_{n}^{\prime}} \mathbb{P}\left(\frac{\operatorname{LIS}\left(\left(Y_{s_{n}, 2}\right)_{\boldsymbol{b}},\left(Y_{s_{n}, 2}^{\prime}\right)_{\boldsymbol{b}^{\prime}}\right)}{\sqrt{s_{n} \rho(R)}}>2 e^{-\Delta x \beta / 2}-\epsilon \mid E_{n}\left(A_{2}, A_{2}^{\prime}\right)\right) \\
= & \sum_{\left|A_{2} \cap A_{2}^{\prime}\right| \geq k_{n}^{\prime}} \mathbb{P}\left(\frac{\operatorname{LIS}\left(\left(Y_{s_{n}, 2}\right)_{\boldsymbol{b}},\left(Y_{s_{n}, 2}^{\prime}\right)_{\boldsymbol{b}^{\prime}}\right)}{\sqrt{s_{n} \rho(R)}}>2 e^{-\Delta x \beta / 2}-\epsilon\right) \times \mathbb{P}\left(E_{n}\left(A_{n}, A_{2}, A_{2}^{\prime}\right)\right)
\end{aligned}
$$




$$
\begin{aligned}
& \geq \sum_{\left|A_{2} \cap A_{2}^{\prime}\right| \geq k_{n}^{\prime}} \mu_{n, 1 / q_{s_{n}}}\left(\frac{\operatorname{LIS}\left(\eta_{\bar{b}}\right)}{\sqrt{s_{n} \rho(R)}}>2 e^{-\Delta x \beta / 2}-\epsilon\right) \times \mathbb{P}\left(E_{n}\left(A_{2}, A_{2}^{\prime}\right)\right) \\
& =\sum_{\left|A_{2} \cap A_{2}^{\prime}\right| \geq k_{n}^{\prime}} \mu_{n, 1 / q_{s_{n}}}\left(\frac{\operatorname{LIS}\left(\eta_{\bar{b}}\right)}{\sqrt{s_{n}(\rho(R)-\delta)}}>\frac{\sqrt{\rho(R)}}{\sqrt{\rho(R)-\delta}}\left(2 e^{-\Delta x \beta / 2}-\epsilon\right)\right) \\
& \geq \sum_{\left|A_{2} \cap A_{2}^{\prime}\right| \geq k_{n}^{\prime}} \mu_{n, 1 / q_{s_{n}}}\left(\frac{\operatorname{LIS}\left(\eta_{\bar{b}}\right)}{\sqrt{k_{n}^{\prime}}}>\left(1+\epsilon_{1}\right)\left(2 e^{-\Delta x \beta / 2}-\epsilon\right)\right) \times \mathbb{P}\left(E_{n}\left(A_{2}, A_{2}^{\prime}, A_{2}^{\prime}\right)\right) \\
& \geq(1-\epsilon) \times \sum_{\left|A_{2} \cap A_{2}^{\prime}\right| \geq k_{n}^{\prime}} \mathbb{P}\left(E_{n}\left(A_{2}, A_{2}^{\prime}\right)\right) \\
& =(1-\epsilon) \times \mathbb{P}_{s_{n}}\left(|\boldsymbol{z}(\pi, \tau) \cap R| \geq k_{n}^{\prime}\right) \\
& =(1-\epsilon) \times \mathbb{P}_{s_{n}}\left(|\boldsymbol{z}(\pi, \tau) \cap R| \geq s_{n}(\rho(R)-\delta)\right) \\
& >(1-\epsilon)^{2} .
\end{aligned}
$$

Here $\mathbb{P}$ denotes the probability space on which $\left(X_{\boldsymbol{c}_{s_{n}}}, Y_{s_{n}, 1}, Y_{s_{n}, 2}, Y_{s_{n}, 3}\right)$ and $\left(X_{\boldsymbol{c}_{s_{n}}^{\prime}}, Y_{s_{n}, 1}^{\prime}, Y_{s_{n}, 2}^{\prime}, Y_{s_{n}, 3}^{\prime}\right)$ are defined. The first equality follows by (3.11). The second equality follows by independence of $\left(X_{\boldsymbol{c}_{s_{n}}}, X_{\boldsymbol{c}_{s_{n}}^{\prime}}\right)$ and $\left(Y_{s_{n}, 2}, Y_{s_{n}, 2}^{\prime}\right)$. The second inequality follows by Lemma 3.2.13, since $Y_{s_{n}, 2}$ and $Y_{s_{n}, 2}^{\prime}$ are independent with $Y_{s_{n}, 2} \sim \mu_{n, q_{s_{n}}}$. The third inequality follows by (3.24) and the fact that $k_{n}^{\prime}=\left\lceil s_{n}(\rho(R)-\delta)\right\rceil \geq$ $s_{n}(\rho(R)-\delta)$. The fourth inequality follows by (3.26) and the fact that $\overline{\boldsymbol{b}}$ has the same dimension as of $\boldsymbol{b}$ which equals to $\left|A_{2} \cap A_{2}^{\prime}\right|$. The last inequality follows by (3.27). Hence, (3.14) follows and this completes the proof of Lemma 3.3.4.

The following lemma establishes certain degree of smoothness of the densities $u$ and $\rho$ defined in Lemma 2.3.1.

Lemma 3.3.5. The density functions $u(x, y, \beta)$ defined in (1.3) and $\rho(x, y)$ defined in (1.6) satisfy the following,

(a) $e^{-|\beta|} \leq u(x, y, \beta) \leq e^{|\beta|}, e^{-|\beta|-|\gamma|} \leq \rho(x, y) \leq e^{|\beta|+|\gamma|}$,

(b) $u(x, y, \beta) \in C_{b}^{1}, \rho(x, y) \in C_{b}^{1}$,

(c) $\max \left(\left|\frac{\partial u}{\partial x}\right|,\left|\frac{\partial u}{\partial y}\right|\right) \leq|\beta| e^{|\beta|}$, 
(d) $\max \left(\left|\frac{\partial \rho}{\partial x}\right|,\left|\frac{\partial \rho}{\partial y}\right|\right) \leq(|\beta|+|\gamma|) e^{|\beta|+|\gamma|}$

where $(x, y) \in[0,1] \times[0,1]$.

Proof. First we show that $e^{-|\beta|} \leq u(x, y, \beta) \leq e^{|\beta|}$ for any $0 \leq x, y \leq 1$. Here we assume $\beta>0$. The proof for the case when $\beta<0$ is similar. By (1.3), we have

$$
\begin{aligned}
u(x, y, \beta) & =\frac{(\beta / 2) \sinh (\beta / 2)}{\left(e^{\beta / 4} \cosh (\beta[x-y] / 2)-e^{-\beta / 4} \cosh (\beta[x+y-1] / 2)\right)^{2}} \\
& =\frac{\beta\left(e^{\beta}-1\right)}{\left(2 e^{\beta / 2} \cosh (\beta[x-y] / 2)-2 \cosh (\beta[x+y-1] / 2)\right)^{2}} .
\end{aligned}
$$

Since $-1 \leq x-y \leq 1$ and $-1 \leq x+y-1 \leq 1$, we have

$$
\begin{aligned}
2 e^{\beta / 2} & \leq 2 e^{\beta / 2} \cosh (\beta[x-y] / 2) \leq e^{\beta}+1 \\
2 & \leq 2 \cosh (\beta[x+y-1] / 2) \leq e^{\beta / 2}+e^{-\beta / 2} .
\end{aligned}
$$

Since $e^{\beta / 2}+e^{-\beta / 2}<2 e^{\beta / 2}$, from (3.29) and (3.30), we have

$$
e^{\beta / 2}-e^{-\beta / 2} \leq 2 e^{\beta / 2} \cosh (\beta[x-y] / 2)-2 \cosh (\beta[x+y-1] / 2) \leq e^{\beta}-1
$$

By (3.28) and (3.31), it follows that

$$
\frac{\beta}{e^{\beta}-1} \leq u(x, y, \beta) \leq \frac{\beta\left(e^{\beta}-1\right)}{\left(e^{\beta / 2}-e^{-\beta / 2}\right)^{2}} .
$$

It is easily verified that

$$
\begin{gathered}
\frac{\beta}{e^{\beta}-1} \geq e^{-\beta} \Longleftrightarrow e^{-\beta} \geq 1-\beta, \\
\frac{\beta\left(e^{\beta}-1\right)}{\left(e^{\beta / 2}-e^{-\beta / 2}\right)^{2}} \leq e^{\beta} \Longleftrightarrow\left(e^{\beta}-1\right)\left(e^{\beta}-1-\beta\right) \geq 0 .
\end{gathered}
$$

By the inequality $e^{x} \geq 1+x$, the right-hand side of (3.33) and (3.34) hold. It follows from (3.32) and the left-hand side of (3.33) and (3.34) that

$$
e^{-\beta} \leq u(x, y, \beta) \leq e^{\beta}, \quad \forall 0 \leq x, y \leq 1
$$

By the definition of $\rho(x, y)$, it follows trivially that

$$
e^{-|\beta|-|\gamma|} \leq \rho(x, y) \leq e^{|\beta|+|\gamma|}, \quad \forall 0 \leq x, y \leq 1
$$


In [26], Starr shows that $\frac{\partial^{2} \ln u(x, y, \beta)}{\partial x \partial y}=2 \beta u(x, y, \beta)$. Thus

$$
\int_{0}^{x} u(t, y, \beta) d t=\frac{1}{2 \beta}\left(\frac{\partial \ln u(x, y, \beta)}{\partial y}-\frac{\partial \ln u(0, y, \beta)}{\partial y}\right) .
$$

By direct calculation, we have $u(1, y, \beta)=\frac{\beta e^{\beta y}}{e^{\beta}-1}, u(0, y, \beta)=\frac{\beta e^{-\beta y}}{1-e^{-\beta}}$. Therefore, we get $\frac{\partial \ln u(1, y, \beta)}{\partial y}=\beta$ and $\frac{\partial \ln u(0, y, \beta)}{\partial y}=-\beta$. By (3.35), it follows that

$$
\frac{\partial u(x, y, \beta)}{\partial y}=2 \beta u(x, y, \beta)\left(\int_{0}^{x} u(t, y, \beta) d t-\frac{1}{2}\right)
$$

and

$$
\int_{0}^{x} u(t, y, \beta) d t \leq \int_{0}^{1} u(t, y, \beta) d t=1 .
$$

From (3.36) and (3.37), we get

$$
\left|\frac{\partial u}{\partial y}\right| \leq|\beta| u(x, y, \beta) \leq|\beta| e^{|\beta|} .
$$

Since $u(x, y, \beta)$ is uniformly continuous on $[0,1] \times[0,1], \int_{0}^{x} u(t, y, \beta) d t$ is also continuous on $[0,1] \times[0,1]$. Hence, by (3.36), $\frac{\partial u}{\partial y}$ is bounded and continuous on $[0,1] \times[0,1]$. Similar argument can be made for $\frac{\partial u}{\partial x}$. Thus we have shown that $u(x, y, \beta) \in C_{b}^{1}$ and

$$
\max \left(\left|\frac{\partial u}{\partial x}\right|,\left|\frac{\partial u}{\partial y}\right|\right) \leq|\beta| e^{|\beta|} .
$$

Next, since $\left|\frac{\partial u(x, t, \beta)}{\partial x} \cdot u(t, y, \gamma)\right| \leq|\beta| e^{|\beta|+|\gamma|}$ for any $0 \leq x, y, t \leq 1$, by dominated convergence theorem, we have

$$
\frac{\partial \rho(x, y)}{\partial x}=\frac{\partial}{\partial x}\left(\int_{0}^{1} u(x, t, \beta) u(t, y, \gamma) d t\right)=\int_{0}^{1} \frac{\partial u(x, t, \beta)}{\partial x} u(t, y, \gamma) d t
$$

Hence, $\left|\frac{\partial \rho}{\partial x}\right| \leq|\beta| e^{|\beta|+|\gamma|}$. Moreover, $\frac{\partial u(x, t, \beta)}{\partial x} \cdot u(t, y, \gamma)$ as a function of $x, y, t$ is uniformly continuous on $[0,1] \times[0,1] \times[0,1]$. Thus, by $(3.39)$, $\frac{\partial \rho}{\partial x}$ is continuous on $[0,1] \times[0,1]$. By a similar argument, it can be shown that $\frac{\partial \rho}{\partial y}$ is continuous on $[0,1] \times[0,1]$, and $\left|\frac{\partial \rho}{\partial y}\right| \leq|\gamma| e^{|\beta|+|\gamma|}$. Therefore, $\rho(x, y) \in C_{b}^{1}$ and

$$
\max \left(\left|\frac{\partial \rho}{\partial x}\right|,\left|\frac{\partial \rho}{\partial y}\right|\right) \leq(|\beta|+|\gamma|) e^{|\beta|+|\gamma|}
$$


The next lemma shows that for any non-decreasing curve in the unit square, in a strip of small width around it, with probability approaching to 1, there exists an increasing subsequence whose length can be bounded from below. The proof of Lemma 3.3.7 uses similar arguments as in the proof of Lemma 8 in [12]. Before stating the lemma, we need the following notation.

Definition 3.3.6. Let $B_{\nearrow}$ be the set of nondecreasing, right continuous functions $\phi$ : $[0,1] \rightarrow[0,1]$. For $\phi \in B_{\nearrow}$, we have $\phi(x)=\int_{0}^{x} \dot{\phi}(t) d t+\phi_{s}(x)$, where $\phi_{s}$ is singular and has a zero derivative almost everywhere. Define function $J: B_{\nearrow} \rightarrow \mathbb{R}$,

$$
J(\phi):=\int_{0}^{1} \sqrt{\dot{\phi}(x) \rho(x, \phi(x))} d x \quad \text { and } \quad \bar{J}:=\sup _{\phi \in B_{\nearrow}} J(\phi) .
$$

Here $\rho(x, y)$ is the density defined in (1.6).

Remark. By Theorems 3 and 4 in [12] it follows from Lemma 3.3 .5 (a) and (b), that

$$
\sup _{\phi \in B_{\nearrow}} J(\phi)=\sup _{\phi \in B_{\nearrow}^{1}} J(\phi)
$$

where $B_{\nearrow}^{1}$ is defined in Theorem 3. Hence we use the same notation $\bar{J}$ to denote the supremum over $B \nearrow$.

Lemma 3.3.7. Under the same conditions as in Theorem 3, for any $\phi \in B_{\nearrow}^{1}$ and any $\delta, \epsilon>0$, define the event

$$
\begin{aligned}
E_{n}:=\{( & (\pi, \tau) \in S_{n} \times S_{n}: \exists \text { an increasing subsequence of }\left\{\left(\frac{\pi(i)}{n}, \frac{\tau(i)}{n}\right)\right\}_{i \in[n]} \\
& \text { which is wholly contained in the } \delta \text { neighborhood of } \phi(\cdot) \\
& \text { and the length of which is greater than } 2 J(\phi)(1-\epsilon) \sqrt{n}\} .
\end{aligned}
$$

Here we say a point $(x, y)$ is in the $\delta$ neighborhood of $\phi$ if $\phi(x)-\delta<y<\phi(x)+\delta$. Then

$$
\lim _{n \rightarrow \infty} \mathbb{P}_{n}\left(E_{n}\right)=1
$$


Proof. Given $\delta, \epsilon>0$, fix an integer $K$. Let $\Delta x:=1 / K$. Let $x_{i}:=i \Delta x$ and $y_{i}:=\phi\left(x_{i}\right)$ for $i \in[K]$. Let $x_{0}:=0, y_{0}:=0$. Define the rectangles $R_{i}:=\left[x_{i-1}, x_{i}\right] \times\left[y_{i-1}, y_{i}\right]$ for $i \in[K]$. Since $\phi$ is in $C_{b}^{1}$, for any $0<\delta^{\prime}<1$, we can choose $K$ large enough such that

$$
\begin{aligned}
& \max _{i}\left(y_{i}-y_{i-1}\right)<\delta, \quad e^{-\Delta x|\beta| / 2}>1-\delta^{\prime}, \quad \Delta x|\beta|<\ln 2 \\
& \max _{i} \max _{x, y \in R_{i}} \max \left(\frac{\rho(x, y)}{\rho\left(x_{i}, y_{i}\right)}, \frac{\rho\left(x_{i}, y_{i}\right)}{\rho(x, y)}\right)<\frac{1}{1-\delta^{\prime}}
\end{aligned}
$$

and

$$
\sum_{i=1}^{K} \sqrt{\rho\left(x_{i}, y_{i}\right)\left(y_{i}-y_{i-1}\right) \Delta x}>\left(1-\delta^{\prime}\right) J(\phi) .
$$

(3.41) follows from the uniform continuity of $\rho(x, y)$ on $[0,1] \times[0,1]$ and the fact that $\rho(x, y)$ is bounded away from 0, which is proved in Lemma 3.3.5 (a). (3.42) follows since

$$
\begin{aligned}
& \lim _{K \rightarrow \infty} \sum_{i=1}^{K} \sqrt{\rho\left(x_{i}, y_{i}\right)\left(y_{i}-y_{i-1}\right) \Delta x} \\
= & \lim _{K \rightarrow \infty} \sum_{i=1}^{K} \sqrt{\rho\left(x_{i}, y_{i}\right) \frac{y_{i}-y_{i-1}}{x_{i}-x_{i-1}}} \Delta x \\
= & J(\phi) .
\end{aligned}
$$

Here the last equality follows from the definition of Riemann integral, the mean value theorem and the fact that $\phi \in C_{b}^{1}$.

Next, for any $i \in[K]$, define $\rho\left(R_{i}\right):=\iint_{R_{i}} \rho(x, y) d x d y$. By (3.41), we have

$$
\frac{\rho\left(R_{i}\right)}{1-\delta^{\prime}}>\rho\left(x_{i}, y_{i}\right)\left(y_{i}-y_{i-1}\right) \Delta x \text {. }
$$

Hence, for any $i \in[K]$, we have

$$
\frac{l_{R_{i}}(\pi, \tau)}{2 \sqrt{n \rho\left(x_{i}, y_{i}\right)\left(y_{i}-y_{i-1}\right) \Delta x}} \geq \frac{l_{R_{i}}(\pi, \tau) \sqrt{1-\delta^{\prime}}}{2 \sqrt{n \rho\left(R_{i}\right)}} .
$$

By fixing the $\epsilon$ in Lemma 3.3.4 to be $2 \delta^{\prime}$, we have

$$
\lim _{n \rightarrow \infty} \mathbb{P}_{n}\left(\frac{l_{R_{i}}(\pi, \tau)}{\sqrt{n \rho\left(R_{i}\right)}}>2 e^{-\Delta x|\beta| / 2}-2 \delta^{\prime}\right)=1 .
$$


Moreover,

$$
\begin{aligned}
& \mathbb{P}_{n}\left(\frac{l_{R_{i}}(\pi, \tau)}{2 \sqrt{n \rho\left(x_{i}, y_{i}\right)\left(y_{i}-y_{i-1}\right) \Delta x}}>\left(1-2 \delta^{\prime}\right) \sqrt{1-\delta^{\prime}}\right) \\
\geq & \mathbb{P}_{n}\left(\frac{l_{R_{i}}(\pi, \tau)}{2 \sqrt{n \rho\left(R_{i}\right)}}>1-2 \delta^{\prime}\right) \\
\geq & \mathbb{P}_{n}\left(\frac{l_{R_{i}}(\pi, \tau)}{\sqrt{n \rho\left(R_{i}\right)}}>2 e^{-\Delta x|\beta| / 2}-2 \delta^{\prime}\right) .
\end{aligned}
$$

The first inequality follows by (3.43), and the second inequality follows by (3.40), since

$$
2 e^{-\Delta x|\beta| / 2}-2 \delta^{\prime}>2\left(1-\delta^{\prime}\right)-2 \delta^{\prime}=2\left(1-2 \delta^{\prime}\right) .
$$

Hence, by (3.44) and (3.45), we get

$$
\lim _{n \rightarrow \infty} \mathbb{P}_{n}\left(\frac{l_{R_{i}}(\pi, \tau)}{2 \sqrt{n \rho\left(x_{i}, y_{i}\right)\left(y_{i}-y_{i-1}\right) \Delta x}}>\left(1-2 \delta^{\prime}\right) \sqrt{1-\delta^{\prime}}\right)=1,
$$

for any $i \in[K]$.

Note that by concatenating the increasing subsequences of $\left\{\left(\frac{\pi(i)}{n}, \frac{\tau(i)}{n}\right)\right\}_{i \in[n]}$ in each $R_{i}$ we get a increasing subsequence in $[0,1] \times[0,1]$ which is wholly contained in a $\delta$ neighborhood of $\phi$. Combining (3.42) and (3.46), it follows that, with probability converging to 1 as $n \rightarrow \infty$, there exists an increasing subsequence of $\left\{\left(\frac{\pi(i)}{n}, \frac{\tau(i)}{n}\right)\right\}_{i \in[n]}$ in a $\delta$ neighborhood of $\phi$ whose length is at least

$$
\sum_{i=1}^{K} 2 \sqrt{n}\left(1-2 \delta^{\prime}\right) \sqrt{1-\delta^{\prime}} \sqrt{\rho\left(x_{i}, y_{i}\right)\left(y_{i}-y_{i-1}\right) \Delta x}>2 \sqrt{n}\left(1-2 \delta^{\prime}\right)\left(1-\delta^{\prime}\right)^{\frac{3}{2}} J(\phi) .
$$

The lemma follows since we can choose $\delta^{\prime}$ small enough in the first place such that $\left(1-2 \delta^{\prime}\right)\left(1-\delta^{\prime}\right)^{\frac{3}{2}}>1-\epsilon$.

Definition 3.3.8. Given $K, L \in \mathbb{N}$ and multi-indices $\boldsymbol{b}=\left(b_{0}, b_{1}, \ldots, b_{K}\right)$ such that $0=b_{0} \leq b_{1} \leq \cdots \leq b_{K}=K L-1$, for any $i \in[K]$, define the rectangle $R_{i}:=$ $((i-1) \Delta x, i \Delta x] \times\left(b_{i-1} \Delta y,\left(b_{i}+1\right) \Delta y\right]$, where $\Delta x:=\frac{1}{K}$ and $\Delta y:=\frac{1}{K L}$. Let $M_{i}:=$ $\sup _{(x, y) \in R_{i}} \rho(x, y)$ and $m_{i}:=\inf _{(x, y) \in R_{i}} \rho(x, y)$. Define

$$
J_{\boldsymbol{b}}^{K, L}:=\sum_{i=1}^{K} \sqrt{M_{i}\left(b_{i}-b_{i-1}+1\right) \Delta x \Delta y}
$$




\section{Lemma 3.3.9.}

$$
\varlimsup_{\substack{K \rightarrow \infty \\ L \rightarrow \infty}} \max _{\boldsymbol{b}} J_{\boldsymbol{b}}^{K, L} \leq \bar{J}
$$

where $\bar{J}$ is defined in Definition 3.3.6, and the maximum is taken over all $\boldsymbol{b}=\left(b_{0}, b_{1}, \ldots, b_{K}\right)$ such that $0=b_{0} \leq b_{1} \leq \cdots \leq b_{K}=K L-1$.

Proof. Let $M$ be an upper bound of $\rho(x, y)$. In the context of Definition 3.3.8, let $\phi_{\boldsymbol{b}}(x)$ be the piecewise linear function on $[0,1]$ such that $\phi_{\boldsymbol{b}}(i \Delta x)=b_{i} \Delta y, i=0,1, \ldots, K$. From the two definitions above, we have

$$
\begin{aligned}
J\left(\phi_{\boldsymbol{b}}\right) & =\int_{0}^{1} \sqrt{\dot{\phi}_{\boldsymbol{b}}(x) \rho\left(x, \phi_{\boldsymbol{b}}(x)\right)} d x \\
& =\sum_{i=1}^{K} \int_{(i-1) \Delta x}^{i \Delta x} \sqrt{\dot{\phi}_{\boldsymbol{b}}(x) \rho\left(x, \phi_{\boldsymbol{b}}(x)\right)} d x \\
& =\sum_{i=1}^{K} \int_{(i-1) \Delta x}^{i \Delta x} \sqrt{\frac{\left(b_{i}-b_{i-1}\right) \Delta y}{\Delta x} \cdot \rho\left(x, \phi_{\boldsymbol{b}}(x)\right)} d x \\
& \geq \sum_{i=1}^{K} \int_{(i-1) \Delta x}^{i \Delta x} \sqrt{\frac{\left(b_{i}-b_{i-1}\right) \Delta y}{\Delta x} \cdot m_{i}} d x \\
& =\sum_{i=1}^{K} \sqrt{m_{i}\left(b_{i}-b_{i-1}\right) \Delta x \Delta y} \\
& \geq \sum_{i=1}^{K} \sqrt{M_{i}\left(b_{i}-b_{i-1}\right) \Delta x \Delta y}-\sum_{i=1}^{K} \sqrt{\left(M_{i}-m_{i}\right)\left(b_{i}-b_{i-1}\right) \Delta x \Delta y}
\end{aligned}
$$

Here the last inequality follows since, for $a, b \geq 0, \sqrt{a}+\sqrt{b} \geq \sqrt{a+b}$. Moreover,

$$
\begin{aligned}
& \sum_{i=1}^{K} \sqrt{M_{i}\left(b_{i}-b_{i-1}\right) \Delta x \Delta y} \\
= & J_{\boldsymbol{b}}^{K, L}-\sum_{i=1}^{K}\left(\sqrt{M_{i}\left(b_{i}-b_{i-1}+1\right) \Delta x \Delta y}-\sqrt{M_{i}\left(b_{i}-b_{i-1}\right) \Delta x \Delta y}\right) \\
= & J_{\boldsymbol{b}}^{K, L}-\sum_{i=1}^{K} \frac{M_{i} \Delta x \Delta y}{\sqrt{M_{i}\left(b_{i}-b_{i-1}+1\right) \Delta x \Delta y}+\sqrt{M_{i}\left(b_{i}-b_{i-1}\right) \Delta x \Delta y}} \\
\geq & J_{\boldsymbol{b}}^{K, L}-\sum_{i=1}^{K} \frac{M_{i} \Delta x \Delta y}{\sqrt{M_{i}\left(b_{i}-b_{i-1}+1\right) \Delta x \Delta y}} \\
\geq & J_{\boldsymbol{b}}^{K, L}-\sum_{i=1}^{K} \frac{M_{i} \Delta x \Delta y}{\sqrt{M_{i} \Delta x \Delta y}}
\end{aligned}
$$




$$
\begin{aligned}
& \geq J_{\boldsymbol{b}}^{K, L}-\sqrt{M} \sum_{i=1}^{K} \sqrt{\Delta x \Delta y} \\
& =J_{\boldsymbol{b}}^{K, L}-\sqrt{\frac{M}{L}} .
\end{aligned}
$$

Next, define

$$
\begin{aligned}
& D_{1}(\boldsymbol{b}):=\left\{i \in[K]:\left(b_{i}-b_{i-1}+1\right) \Delta y \leq \sqrt[3]{\Delta x}\right\} \\
& D_{2}(\boldsymbol{b}):=\left\{i \in[K]:\left(b_{i}-b_{i-1}+1\right) \Delta y>\sqrt[3]{\Delta x}\right\}
\end{aligned}
$$

For $i \in D_{1}(\boldsymbol{b})$, the height of $R_{i}$ is no greater than $\sqrt[3]{\Delta x}$, and for $i \in D_{2}(\boldsymbol{b})$, the height of $R_{i}$ is greater than $\sqrt[3]{\Delta x}$. To bound the cardinality of $D_{2}(\boldsymbol{b})$, we have

$$
\begin{aligned}
\left|D_{2}(\boldsymbol{b})\right| \sqrt[3]{\Delta x} & \leq \sum_{i \in D_{2}(\boldsymbol{b})}\left(b_{i}-b_{i-1}+1\right) \Delta y \\
& \leq \sum_{i \in D_{2}(\boldsymbol{b})}\left(b_{i}-b_{i-1}\right) \Delta y+\left|D_{2}(\boldsymbol{b})\right| \Delta y \\
& \leq \sum_{i=1}^{K}\left(b_{i}-b_{i-1}\right) \Delta y+K \Delta y \\
& \leq 1+\frac{1}{L} \\
& \leq 2 .
\end{aligned}
$$

Given $\epsilon>0$, by the uniform continuity of $\rho(x, y)$ on $[0,1] \times[0,1]$, there exists $K_{0}>0$ such that, for any $K>K_{0}$ and any $i \in D_{1}(\boldsymbol{b})$, we have $M_{i}-m_{i}<\epsilon^{2}$. We can also choose $K_{0}$ sufficiently large such that, for any $K>K_{0}$,

$$
2 \sqrt{M}(\Delta x)^{\frac{1}{6}}<\epsilon .
$$

Thus, for any $K>K_{0}$, we have

$$
\begin{aligned}
& \sum_{i=1}^{K} \sqrt{\left(M_{i}-m_{i}\right)\left(b_{i}-b_{i-1}\right) \Delta x \Delta y} \\
\leq & \sum_{i \in D_{1}(\boldsymbol{b})} \sqrt{\epsilon^{2}\left(b_{i}-b_{i-1}\right) \Delta x \Delta y}+\sum_{i \in D_{2}(\boldsymbol{b})} \sqrt{M\left(b_{i}-b_{i-1}\right) \Delta x \Delta y}
\end{aligned}
$$




$$
\begin{aligned}
& \leq \epsilon \sum_{i=1}^{K} \sqrt{\left(b_{i}-b_{i-1}\right) \Delta x \Delta y}+\sum_{i \in D_{2}(\boldsymbol{b})} \sqrt{M \Delta x} \\
& \leq \epsilon \sqrt{\sum_{i=1}^{K} \Delta x} \sqrt{\sum_{i=1}^{K}\left(b_{i}-b_{i-1}\right) \Delta y}+2 \sqrt{M}(\Delta x)^{\frac{1}{6}} \\
& <\epsilon+\epsilon .
\end{aligned}
$$

Here the second to last inequality follows by Cauchy-Schwarz inequality and (3.49). Let $L_{0}:=\left\lceil\frac{M}{\epsilon^{2}}\right\rceil$. By combining (3.47), (3.48) and (3.51), we get, for any $K>K_{0}$, $L>L_{0}$ and any $\boldsymbol{b}$,

$$
J_{\boldsymbol{b}}^{K, L} \leq J\left(\phi_{\boldsymbol{b}}\right)+\sqrt{\frac{M}{L}} \leq J\left(\phi_{\boldsymbol{b}}\right)+3 \epsilon \leq \bar{J}+3 \epsilon .
$$

Here the last inequality follows from the fact that $\phi_{\boldsymbol{b}} \in B_{\nearrow}$ and Definition 3.3.6.

Definition 3.3.10. In the context of Definition 3.3.8, we call a sequence of points $\left(z_{1}, \ldots, z_{m}\right)$ with $z_{i}=\left(x_{i}, y_{i}\right)$ a $\boldsymbol{b}$-increasing sequence if the following two conditions are satisfied.

(a) $\left(z_{1}, \ldots, z_{m}\right)$ is an increasing sequence, that is $x_{i}<x_{i+1}$ and $y_{i}<y_{i+1}$ for all $i \in[m-1]$.

(b) Every point in the sequence is contained in some rectangle $R_{j}$ with $j \in[K]$. In other words, $(j-1) \Delta x<x_{i} \leq j \Delta x$ implies $b_{j-1} \Delta y<y_{i} \leq\left(b_{j}+1\right) \Delta y$.

Given a collection of points $\boldsymbol{z}=\left\{z_{i}\right\}_{i \in[n]}$, let $L I S_{\boldsymbol{b}}(\boldsymbol{z})$ denote the length of the longest $\boldsymbol{b}$-increasing subsequence of $\boldsymbol{z}$. That is

$$
L I S_{\boldsymbol{b}}(\boldsymbol{z}):=\max \left\{m: \exists\left(i_{1}, i_{2}, \ldots, i_{m}\right)\right.
$$

such that $\left(z_{i_{1}}, z_{i_{2}}, \ldots, z_{i_{m}}\right)$ is a $\boldsymbol{b}$-increasing sequence $\}$.

Here we do not require $i_{j}<i_{j+1}$.

Lemma 3.3.11. Under the same conditions as in Lemma 2.3.1, for any $\delta>0$, there exist $K_{0}, L_{0}$ such that, for any $K>K_{0}, L>L_{0}$ and any $\boldsymbol{b}=\left(b_{0}, b_{1}, \ldots, b_{K}\right)$ with $0=b_{0} \leq b_{1} \leq \cdots \leq b_{K}=K L-1$,

$$
\lim _{n \rightarrow \infty} \mathbb{P}_{n}\left(L I S_{\boldsymbol{b}}(\boldsymbol{z}(\pi, \tau))>2 \sqrt{n}(\bar{J}+\delta)\right)=0
$$


Here $\boldsymbol{z}(\pi, \tau):=\left\{\left(\frac{\pi(i)}{n}, \frac{\tau(i)}{n}\right)\right\}_{i \in[n]}$.

Proof. Given $\delta>0$, by Lemma 3.3.9, there exist $K_{1}, L_{1}>0$ such that, for any $K>$ $K_{1}, L>L_{1}$ and any $\boldsymbol{b}=\left(b_{0}, b_{1}, \ldots, b_{K}\right)$ with $0=b_{0} \leq b_{1} \leq \cdots \leq b_{K}=K L-1$, we have

$$
J_{\boldsymbol{b}}^{K, L}<\bar{J}+\frac{\delta}{2}
$$

Then, we get

$$
\mathbb{P}_{n}\left(\operatorname{LIS}_{\boldsymbol{b}}(\boldsymbol{z}(\pi, \tau))>2 \sqrt{n}(\bar{J}+\delta)\right) \leq \mathbb{P}_{n}\left(\operatorname{LIS}_{\boldsymbol{b}}(\boldsymbol{z}(\pi, \tau))>2 \sqrt{n}\left(J_{\boldsymbol{b}}^{K, L}+\delta / 2\right)\right)
$$

Hence, to show (3.52), it suffices to show that there exists $K_{2}, L_{2}$ such that, for any $K>K_{2}, L>L_{2}$ and any $\boldsymbol{b}$,

$$
\lim _{n \rightarrow \infty} \mathbb{P}_{n}\left(\operatorname{LIS}_{\boldsymbol{b}}(\boldsymbol{z}(\pi, \tau))>2 \sqrt{n}\left(J_{\boldsymbol{b}}^{K, L}+\delta / 2\right)\right)=0
$$

Given $K, L>0$, whose values are to be determined, and any $\boldsymbol{b}=\left(b_{0}, \ldots, b_{K}\right)$ with $0=b_{0} \leq b_{1} \leq \cdots \leq b_{K}=K L-1$, we inherit all the notations introduced in Definition 3.3.8. Let $l_{R_{i}}(\pi, \tau)$ denote the length of the longest increasing subsequence of $\boldsymbol{z}(\pi, \tau)$ wholly contained in the rectangle $R_{i}$. For any $i \in[K]$, define

$$
E_{i}(\boldsymbol{b}):=\left\{(\pi, \tau): l_{R_{i}}(\pi, \tau) \geq 2 \sqrt{n}\left(\sqrt{M_{i}\left(b_{i}-b_{i-1}+1\right) \Delta x \Delta y}+\delta \Delta x / 2\right)\right\}
$$

Since $\operatorname{LIS}_{\boldsymbol{b}}(\boldsymbol{z}(\pi, \tau)) \leq \sum_{i=1}^{K} l_{R_{i}}(\pi, \tau)$, we get

$$
\left\{\operatorname{LIS}_{\boldsymbol{b}}(\boldsymbol{z}(\pi, \tau))>2 \sqrt{n}\left(J_{\boldsymbol{b}}^{K, L}+\delta / 2\right)\right\} \subset \bigcup_{i \in[K]} E_{i}(\boldsymbol{b}) .
$$

Hence, to show (3.53), it suffices to show

$$
\lim _{n \rightarrow \infty} \mathbb{P}_{n}\left(E_{i}(\boldsymbol{b})\right)=0, \quad \forall i \in[K]
$$

Since $e^{\Delta x|\beta| / 2}-1=\Theta(\Delta x)$, there exists $K_{2}>0$ such that, for any $K>K_{2}$, we have

$$
e^{\Delta x|\beta| / 2}<1+\frac{\delta \sqrt{\Delta x}}{2 \sqrt{M}} \quad \text { and } \quad \Delta x|\beta|<\ln 2
$$


Here $M:=\sup _{0 \leq x, y \leq 1} \rho(x, y)$. Moreover, for any $i \in[K]$,

$$
\begin{aligned}
& \mathbb{P}_{n}\left(E_{i}(\boldsymbol{b})\right) \\
\leq & \mathbb{P}_{n}\left(l_{R_{i}}(\pi, \tau) \geq 2 \sqrt{n} \sqrt{M_{i}\left(b_{i}-b_{i-1}+1\right) \Delta x \Delta y}\left(1+\frac{\delta \Delta x}{2 \sqrt{M \Delta x}}\right)\right) \\
\leq & \mathbb{P}_{n}\left(l_{R_{i}}(\pi, \tau) \geq 2 \sqrt{n \rho\left(R_{i}\right)}\left(1+\frac{\delta \sqrt{\Delta x}}{2 \sqrt{M}}\right)\right) .
\end{aligned}
$$

Here the first inequality follows since $\left(b_{i}-b_{i-1}+1\right) \Delta y \leq 1$ and $M_{i} \leq M$. The second one follows since

$$
M_{i}\left(b_{i}-b_{i-1}+1\right) \Delta x \Delta y \geq \int_{R_{i}} \rho(x, y) d x d y=\rho\left(R_{i}\right) .
$$

Hence, combining (3.55), (3.56) and Lemma 3.3.4, we get, for any $K>K_{2}, L>0$ and any $\boldsymbol{b}$,

$$
\lim _{n \rightarrow \infty} \mathbb{P}_{n}\left(E_{i}(\boldsymbol{b})\right)=0, \quad \forall i \in[K] .
$$

Thus, (3.54) as well as the lemma follow.

Proof of Theorem 3. Lemma 1.1.4, if $\pi \sim \mu_{n, q}, \pi^{-1}$ has the same distribution $\mu_{n, q}$. Hence, if $(\pi, \tau) \sim \mu_{n, q} \times \mu_{n, q^{\prime}},\left(\pi^{-1}, \tau^{-1}\right)$ has the same distribution $\mu_{n, q} \times \mu_{n, q^{\prime}}$. Thus, by Corollary 3.1.4, to prove Theorem 3, it suffices to show

$$
\lim _{n \rightarrow \infty} \mathbb{P}_{n}\left(\left|\frac{\operatorname{LIS}(\boldsymbol{z}(\pi, \tau))}{\sqrt{n}}-2 \bar{J}\right|<\epsilon\right)=1,
$$

for any $\epsilon>0$. Here we use the trivial fact that $\operatorname{LIS}(\pi, \tau)=\operatorname{LIS}(\boldsymbol{z}(\pi, \tau))$.

By Lemma 3.3.7 and the definition of $\bar{J}$, we have

$$
\lim _{n \rightarrow \infty} \mathbb{P}_{n}\left(\frac{\operatorname{LIS}(\boldsymbol{z}(\pi, \tau))}{\sqrt{n}}>2 \bar{J}-\epsilon\right)=1 .
$$

To show the upper bound in (3.57), note that, for any $K, L>0$ and any increasing sequence of points $\left\{\left(x_{j}, y_{j}\right)\right\}_{j \in[n]}$ with $0<x_{j}, y_{j} \leq 1$, there exists a choice of $\boldsymbol{b}=$ $\left(b_{0}, b_{1}, \ldots, b_{K}\right)$ such that $\left\{\left(x_{j}, y_{j}\right)\right\}_{j \in[n]}$ is a $\boldsymbol{b}$ - increasing sequence. Specifically, we can define $\boldsymbol{b}$ as follows. Let $\Delta x:=\frac{1}{K}, \Delta y:=\frac{1}{K L}$.

- Define $b_{0}:=0, b_{K}:=K L-1$. 
- For $i \in[K-1]$, define $b_{i}:=\left\lfloor\max \left\{y_{j}:(i-1) \Delta x<x_{j} \leq i \Delta x\right\} \cdot K L\right\rfloor$.

It can be easily verified that with $\boldsymbol{b}$ thus defined, every point $\left(x_{j}, y_{j}\right)$ is in some rectangle $R_{i}$, where $R_{i}$ is defined in Definition 3.3.8. Hence, we get

$$
\begin{aligned}
& \mathbb{P}_{n}\left(\frac{\operatorname{LIS}(\boldsymbol{z}(\pi, \tau))}{\sqrt{n}}>2 \bar{J}+\epsilon\right) \\
= & \mathbb{P}_{n}\left(\max _{\boldsymbol{b}}\left(\operatorname{LIS}_{\boldsymbol{b}}(\boldsymbol{z}(\pi, \tau))\right)>\sqrt{n}(2 \bar{J}+\epsilon)\right) \\
\leq & \sum_{\boldsymbol{b}} \mathbb{P}_{n}\left(\operatorname{LIS}_{\boldsymbol{b}}(\boldsymbol{z}(\pi, \tau))>\sqrt{n}(2 \bar{J}+\epsilon)\right) .
\end{aligned}
$$

Here, the maximum and summation are taken over all possible $\boldsymbol{b}$ with $0=b_{0} \leq b_{1} \leq$ $\cdots \leq b_{K}=K L-1$. By Lemma 3.3.11, we can choose $K, L$ sufficiently large such that, for any $\boldsymbol{b}$,

$$
\lim _{n \rightarrow \infty} \mathbb{P}_{n}\left(\operatorname{LIS}_{\boldsymbol{b}}(\boldsymbol{z}(\pi, \tau))>\sqrt{n}(2 \bar{J}+\epsilon)\right)=0 .
$$

Hence, by (3.59) and the fact that the number of different choices of $\boldsymbol{b}$ is bounded above by $(K L)^{K}$, we have

$$
\lim _{n \rightarrow \infty} \mathbb{P}_{n}\left(\frac{\operatorname{LIS}(\boldsymbol{z}(\pi, \tau))}{\sqrt{n}}>2 \bar{J}+\epsilon\right)=0 .
$$

(3.57) follows from (3.58) and (3.60)

The following lemma let us solve for the supremum $\bar{J}$ when the underlying density $\rho(x, y)$ satisfies $\rho\left(\frac{x+y}{2}, \frac{x+y}{2}\right) \geq \rho(x, y)$.

Lemma 3.3.12. Given a density $\rho(x, y)$ on $[0,1] \times[0,1]$ such that $\rho(x, y)$ is $C_{b}^{1}$ and $c<\rho(x, y)<C$ for some $C, c>0$, if $\rho(x, y) \leq \rho\left(\frac{x+y}{2}, \frac{x+y}{2}\right)$ for any $0 \leq x, y \leq 1$, then we have

$$
\bar{J}=\int_{0}^{1} \sqrt{\rho(x, x)} d x,
$$

i. e. the supremum of $J(\phi)$ on $B_{\nearrow}$ is attained for $\phi(x)=x$.

Proof. By the remark following Definition 3.3.6, it suffices to show that, for any $\phi \in B_{\nearrow}^{1}$, we have

$$
J(\phi) \leq \int_{0}^{1} \sqrt{\rho(x, x)} d x
$$


Define $g_{\phi}(x):=x+\phi(x)$. Since $\dot{\phi}(x) \geq 0$, we have $\dot{g}_{\phi}(x) \geq 1$. Next, we reparameterize $\phi(x)$ as follows,

$$
t:=\frac{g_{\phi}(x)}{2}=\frac{x+\phi(x)}{2} .
$$

Thus, we have $x=g_{\phi}^{-1}(2 t)$ and $\phi(x)=2 t-x=2 t-g_{\phi}^{-1}(2 t)$ where $t \in[0,1]$. Moreover, since $g_{\phi}(x)$ is strictly increasing, $x$ is strictly increasing as a function of $t$. Hence we have

$$
\rho(x, \phi(x))=\rho\left(g_{\phi}^{-1}(2 t), 2 t-g_{\phi}^{-1}(2 t)\right) \leq \rho(t, t)
$$

Here the last inequality follows since $\rho(x, y) \leq \rho\left(\frac{x+y}{2}, \frac{x+y}{2}\right)$. Next, by taking derivative with respect to $t$ on both sides of (3.62), we have

$$
1=\frac{1}{2}\left(\frac{d x}{d t}+\dot{\phi}(x) \frac{d x}{d t}\right)
$$

By multiplying $2 \frac{d x}{d t}$ on both sides of (3.64), we get

$$
\dot{\phi}(x)\left(\frac{d x}{d t}\right)^{2}=2 \frac{d x}{d t}-\left(\frac{d x}{d t}\right)^{2} \leq 1 .
$$

Hence, by (3.63) and (3.65), we have

$$
\begin{aligned}
J(\phi) & =\int_{0}^{1} \sqrt{\dot{\phi}(x) \rho(x, \phi(x))} d x \\
& \leq \int_{0}^{1} \sqrt{\dot{\phi}(x) \rho(t, t) \cdot \frac{d x}{d t} d t} \\
& =\int_{0}^{1} \sqrt{\rho(t, t) \dot{\phi}(x)\left(\frac{d x}{d t}\right)^{2}} d t \\
& \leq \int_{0}^{1} \sqrt{\rho(t, t)} d t .
\end{aligned}
$$

Therefore, $\bar{J}$ is attained for $\phi(x)=x$.

Proof of Corollary 3. Note that in the special case where $\beta=\gamma$, the density $\rho(x, y)$ in (1.6) is given by

$$
\rho(x, y):=\int_{0}^{1} u(x, t, \beta) \cdot u(t, y, \beta) d t
$$


In this case, we will show that $\rho(x, y) \leq \rho\left(\frac{x+y}{2}, \frac{x+y}{2}\right)$ for any $0 \leq x, y \leq 1$. Hence, by Lemma 3.3.5 and Lemma 3.3.12, $\bar{J}$ defined in Theorem 3 is attained when $\phi(x)=x$. In fact, by direct calculation, it can be shown that

$$
u(x, t, \beta) \cdot u(t, y, \beta) \leq u\left(\frac{x+y}{2}, t, \beta\right) \cdot u\left(t, \frac{x+y}{2}, \beta\right),
$$

for any $0 \leq x, y, t \leq 1$.

By the definition of $u(x, y, \beta)$, we have

$$
\begin{aligned}
& u(x, t, \beta) \cdot u(t, y, \beta) \\
& =\frac{(\beta / 2) \sinh (\beta / 2)}{\left(e^{\beta / 4} \cosh (\beta[x-t] / 2)-e^{-\beta / 4} \cosh (\beta[x+t-1] / 2)\right)^{2}} \\
& \times \frac{(\beta / 2) \sinh (\beta / 2)}{\left(e^{\beta / 4} \cosh (\beta[t-y] / 2)-e^{-\beta / 4} \cosh (\beta[t+y-1] / 2)\right)^{2}} \\
& =\frac{\beta\left(e^{\beta}-1\right)}{\left(2 e^{\beta / 2} \cosh (\beta[x-t] / 2)-2 \cosh (\beta[x+t-1] / 2)\right)^{2}} \\
& \times \frac{\beta\left(e^{\beta}-1\right)}{\left(2 e^{\beta / 2} \cosh (\beta[t-y] / 2)-2 \cosh (\beta[t+y-1] / 2)\right)^{2}} .
\end{aligned}
$$

Considering the term inside the square of the denominator, by using the hyperbolic trigonometric identities,

$$
\begin{aligned}
\cosh (x) \cosh (y) & =(\cosh (x+y)+\cosh (x-y)) / 2, \\
\cosh (x+y) & =\cosh (x) \cosh (y)+\sinh (x) \sinh (y), \\
\cosh (x-y) & =\cosh (x) \cosh (y)-\sinh (x) \sinh (y),
\end{aligned}
$$

we get

$$
\begin{gathered}
\left(2 e^{\beta / 2} \cosh (\beta[x-t] / 2)-2 \cosh (\beta[x+t-1] / 2)\right) \\
\times\left(2 e^{\beta / 2} \cosh (\beta[t-y] / 2)-2 \cosh (\beta[t+y-1] / 2)\right) \\
=2 e^{\beta}(\cosh (\beta[x-y] / 2)+\cosh (\beta[x+y-2 t] / 2)) \\
-2 e^{\beta / 2}(\cosh (\beta[x+y-1] / 2)+\cosh (\beta[x-y-2 t+1] / 2)) \\
-2 e^{\beta / 2}(\cosh (\beta[x-y+2 t-1] / 2)+\cosh (\beta[x+y-1] / 2)) \\
+2(\cosh (\beta[x+y+2 t-2] / 2)+\cosh (\beta[x-y] / 2))
\end{gathered}
$$




$$
=S_{t}^{-}+S_{t}^{+}
$$

Here $S_{t}^{-}$denotes the sum of those terms in the above equation containing the term $x-y$. $S_{t}^{+}$denotes the sum of those which contain the term $x+y$. After further simplification using the identities above, we have

$$
S_{t}^{-}=2 \cosh (\beta[x-y] / 2)\left(e^{\beta}-2 e^{\beta / 2} \cosh (\beta[2 t-1] / 2)+1\right) .
$$

It is easily seen that the minimum of $e^{\beta}-2 e^{\beta / 2} \cosh (\beta[2 t-1] / 2)+1$ for $0 \leq t \leq 1$ is attained when $t=0,1$, and the minimum is 0 . Hence, for any $t \in[0,1]$, $S_{t}^{-}$is minimized when $x=y$. Thus to prove (3.67), it suffices to show that $S_{t}^{+} \geq 0$, since $S_{t}^{-}+S_{t}^{+}$is the term inside the square of the denominator of (3.68). After simplification, we have

$$
\begin{aligned}
S_{t}^{+}=2 e^{\beta}(\cosh (\beta[x+y-1] / 2) \cosh (\beta[2 t-1] / 2) \\
-\sinh (\beta[x+y-1] / 2) \sinh (\beta[2 t-1] / 2)) \\
-4 e^{\beta / 2} \cosh (\beta[x+y-1] / 2) \\
+2(\cosh (\beta[x+y-1] / 2) \cosh (\beta[2 t-1] / 2) \\
+\sinh (\beta[x+y-1] / 2) \sinh (\beta[2 t-1] / 2)) .
\end{aligned}
$$

Next, we make change of variables. Define $r:=e^{\beta(x+y-1) / 2}, s:=e^{\beta(2 t-1) / 2}$. Then, from (3.71), we have

$$
\begin{aligned}
S_{t}^{+}= & \frac{e^{\beta}}{2}\left(\left(r+\frac{1}{r}\right)\left(s+\frac{1}{s}\right)-\left(r-\frac{1}{r}\right)\left(s-\frac{1}{s}\right)\right)-2 e^{\beta / 2}\left(r+\frac{1}{r}\right) \\
& \quad+\frac{1}{2}\left(\left(r+\frac{1}{r}\right)\left(s+\frac{1}{s}\right)+\left(r-\frac{1}{r}\right)\left(s-\frac{1}{s}\right)\right) \\
= & e^{\beta}\left(\frac{r}{s}+\frac{s}{r}\right)-2 e^{\beta / 2}\left(r+\frac{1}{r}\right)+\left(r s+\frac{1}{r s}\right) \\
= & \left(\frac{e^{\beta} r}{s}+r s-2 e^{\beta / 2} r\right)+\left(\frac{e^{\beta} s}{r}+\frac{1}{r s}-\frac{2 e^{\beta / 2}}{r}\right) \\
\geq & 0 .
\end{aligned}
$$


Here the last inequality follows since $x+y \geq 2 \sqrt{x y}$ for any $x, y \geq 0$. We complete the proof of Corollary 3 by showing:

$$
\int_{0}^{1} u(x, t, \beta) \cdot u(t, x, \beta) d t=\frac{\beta(\cosh (\beta / 2)+2 \cosh (\beta[2 x-1] / 2))}{6 \sinh (\beta / 2)},
$$

for $0 \leq x \leq 1$.

By the same change of variables as above, since $y=x$, let $r:=e^{\beta(2 x-1) / 2}$, $s:=e^{\beta(2 t-1) / 2}$. Then, we have

$$
\frac{d t}{d s}=\frac{1}{\frac{d s}{d t}}=\frac{1}{s \beta}
$$

By (3.70), we have,

$$
S_{t}^{-}=2\left(e^{\beta}-e^{\beta / 2}\left(s+\frac{1}{s}\right)+1\right) .
$$

Then, by (3.72) and (3.75), it can be easily verified that

$$
r s\left(S_{t}^{+}+S_{t}^{-}\right)=\left(e^{\beta / 2}(r+s)-(r s+1)\right)^{2} .
$$

Hence, we have

$$
\begin{aligned}
& \int_{0}^{1} u(x, t, \beta) \cdot u(t, x, \beta) d t \\
= & \int_{e^{-\beta / 2}}^{e^{\beta / 2}} \frac{\beta^{2}\left(e^{\beta}-1\right)^{2}}{\left(S_{t}^{+}+S_{t}^{-}\right)^{2}} \frac{1}{s \beta} d s \\
= & \int_{e^{-\beta / 2}}^{e^{\beta / 2}} \frac{\beta\left(e^{\beta}-1\right)^{2} r^{2} s}{\left(r s\left(S_{t}^{+}+S_{t}^{-}\right)\right)^{2}} d s \\
= & \int_{e^{-\beta / 2}}^{e^{\beta / 2}} \frac{\beta\left(e^{\beta}-1\right)^{2} r^{2} s}{\left(e^{\beta / 2}(r+s)-(r s+1)\right)^{4}} d s \\
= & \beta\left(e^{\beta}-1\right)^{2} r^{2} \int_{e^{-\beta / 2}}^{e^{\beta / 2}} \frac{s}{\left(\left(e^{\beta / 2}-r\right) s+e^{\beta / 2} r-1\right)^{4}} d s \\
= & \beta\left(e^{\beta}-1\right)^{2} e^{\beta(2 x-1)} \int_{e^{-\beta / 2}}^{e^{\beta / 2}} \frac{s}{\left(e^{\beta / 2}\left(1-e^{\beta(x-1)}\right) s+e^{\beta x}-1\right)^{4}} d s .
\end{aligned}
$$

Here the first equality follows from (3.68), (3.69), (3.74) and change of variables. The third equality follows from (3.76). Then we make another change of variable by defining

$$
w:=\frac{e^{\beta / 2}\left(1-e^{\beta(x-1)}\right) s+e^{\beta x}-1}{e^{\beta}-1},
$$


from which we have

$$
\frac{d s}{d w}=\frac{e^{\beta}-1}{e^{\beta / 2}\left(1-e^{\beta(x-1)}\right)}, \quad \text { and } \quad w= \begin{cases}1 & \text { when } s=e^{\beta / 2} \\ e^{\beta(x-1)} & \text { when } s=e^{-\beta / 2}\end{cases}
$$

Hence, by (3.77), we have

$$
\begin{aligned}
& \int_{0}^{1} u(x, t, \beta) \cdot u(t, x, \beta) d t \\
= & \frac{\beta e^{2 \beta(x-1)}}{\left(e^{\beta}-1\right)\left(1-e^{\beta(x-1)}\right)^{2}} \int_{e^{\beta(x-1)}}^{1} \frac{\left(e^{\beta}-1\right) w-e^{\beta x}+1}{w^{4}} d w \\
= & \left.\frac{\beta e^{2 \beta(x-1)}}{\left(e^{\beta}-1\right)\left(1-e^{\beta(x-1)}\right)^{2}}\left(\frac{1-e^{\beta}}{2 w^{2}}+\frac{e^{\beta x}-1}{3 w^{3}}\right)\right|_{e^{\beta(x-1)}} ^{1} \\
= & \frac{\beta\left(1+e^{\beta}+2 e^{\beta x}+2 e^{-\beta(x-1)}\right)}{6\left(e^{\beta}-1\right)} \\
= & \frac{\beta(\cosh (\beta / 2)+2 \cosh (\beta[2 x-1] / 2))}{6 \sinh (\beta / 2)} .
\end{aligned}
$$

\subsection{More Couplings of Mallows Permutations}

In this section, we will prove the following lemmas. Based on these lemmas, we can construct couplings of pairs of independent Mallows permutations such that there exists ordering of the length of the LCS in terms of the ordering of the underlying parameters.

Lemma 3.4.1. Given $q \in(0,1]$ and $0<q_{1}<q_{2}$, there exists a coupling $\left(X_{1}, Y_{1}, X_{2}, Y_{2}\right)$ such that

(a) $X_{1} \sim \mu_{n, q}, X_{2} \sim \mu_{n, q}, Y_{1} \sim \mu_{n, q_{1}}$ and $Y_{2} \sim \mu_{n, q_{2}}$.

(b) $X_{1}$ and $Y_{1}$ are independent. $X_{2}$ and $Y_{2}$ are independent.

(c) $\operatorname{LCS}\left(X_{1}, Y_{1}\right) \geq \operatorname{LCS}\left(X_{2}, Y_{2}\right)$.

Lemma 3.4.2. Given $q \in(0,1]$ and $q^{\prime}>0$, there exists a coupling $(X, Y, Z)$ such that

(a) $X \sim \mu_{n, q}, Y \sim \mu_{n, q}$ and $Z \sim \mu_{n, q^{\prime}}$. 
(b) $Y$ and $Z$ are independent.

(c) $\operatorname{LIS}(X) \geq \operatorname{LCS}(Y, Z)$.

Lemma 3.4.3. Given $q \geq 1$ and $0<q_{1}<q_{2}$, there exists a coupling $\left(X_{1}, Y_{1}, X_{2}, Y_{2}\right)$ such that

(a) $X_{1} \sim \mu_{n, q}, X_{2} \sim \mu_{n, q}, Y_{1} \sim \mu_{n, q_{1}}$ and $Y_{2} \sim \mu_{n, q_{2}}$.

(b) $X_{1}$ and $Y_{1}$ are independent. $X_{2}$ and $Y_{2}$ are independent.

(c) $\operatorname{LCS}\left(X_{1}, Y_{1}\right) \leq \operatorname{LCS}\left(X_{2}, Y_{2}\right)$.

Lemma 3.4.4. Given $q \geq 1$ and $q^{\prime}>0$, there exists a coupling $(X, Y, Z)$ such that

(a) $X \sim \mu_{n, q}, Y \sim \mu_{n, q}$ and $Z \sim \mu_{n, q^{\prime}}$.

(b) $Y$ and $Z$ are independent.

(c) $\operatorname{LIS}(X) \leq \operatorname{LCS}(Y, Z)$.

The following two lemmas play the key role in the proofs of the four lemmas above.

Lemma 3.4.5. Given $\pi, \tau \in S_{n}$ with $\pi \leq_{R} \tau$, for any $q \in(0,1]$, there exists a coupling $(X, Y)$ such that $X \sim \mu_{n, q}, Y \sim \mu_{n, q}$ and

$$
\operatorname{LCS}(X, \pi) \geq \operatorname{LCS}(Y, \tau)
$$

Lemma 3.4.6. Given $\pi, \tau \in S_{n}$ with $\pi \leq_{R} \tau$, for any $q \geq 1$, there exists a coupling $(X, Y)$ such that $X \sim \mu_{n, q}, Y \sim \mu_{n, q}$ and

$$
\operatorname{LCS}(X, \pi) \leq \operatorname{LCS}(Y, \tau)
$$

Proof of Lemma 3.4.5. The proof of this lemma is similar to the proof of Lemma 3.2.9. Firstly, we claim that it suffices to show the case when $\tau$ covers $\pi$ in $\left(S_{n}, \leq_{R}\right)$, that is $l(\tau)=l(\pi)+1$ and $\tau=\pi \circ s_{i}$ for some $i \in[n-1]$. The claim can be shown 
by induction on the Kendall's tau distance of $\pi$ and $\tau$, i.e., the minimum number of adjacent transpositions multiplied to $\pi$ from the right to get $\tau$. Suppose we have $\pi \leq_{R} \sigma \leq_{R} \tau$ in $S_{n}$. By induction hypothesis there exist two couplings $\left(X^{\prime}, Z\right)$ and $\left(Z^{\prime}, Y^{\prime}\right)$, which are not necessarily defined in the same probability space, such that $X^{\prime}, Z, Z^{\prime}, Y^{\prime}$ have the same marginal distribution $\mu_{n, q}$ and

$$
\operatorname{LCS}\left(X^{\prime}, \pi\right) \geq \operatorname{LCS}(Z, \sigma), \quad \operatorname{LCS}\left(Z^{\prime}, \sigma\right) \geq \operatorname{LCS}\left(Y^{\prime}, \tau\right)
$$

We can construct a new coupling $(X, Y)$ as follows,

- Sample a permutation $\xi \in S_{n}$ according to the distribution $\mu_{n, q}$.

- Sample $X$ according to the induced distribution on $S_{n}$ by the first coupling $\left(X^{\prime}, Z\right)$ conditioned on $Z=\xi$.

- Sample $Y$ according to the induced distribution on $S_{n}$ by the second coupling $\left(Z^{\prime}, Y^{\prime}\right)$ conditioned on $Z^{\prime}=\xi$.

By the law of total probability, it is easily seen that $X \sim \mu_{n, q}$ and $Y \sim \mu_{n, q}$. Also, regardless of which permutation $\xi$ is being sampled in the first step, by (3.78), we have

$$
\operatorname{LCS}(X, \pi) \geq \operatorname{LCS}(\xi, \sigma) \geq \operatorname{LCS}(Y, \tau)
$$

In the remainder of the proof, we assume $\tau=\pi \circ s_{i}$ and $l(\tau)=l(\pi)+1$. Note that, for any $\sigma \in S_{n}$,

$$
\sigma \circ(i, j)=(\sigma(i), \sigma(j)) \circ \sigma
$$

Here $(i, j)$ denotes the transposition of $i$ and $j$. Hence we have

$$
\tau=\pi \circ s_{i}=(\pi(i), \pi(i+1)) \circ \pi
$$

Since $l(\tau)=l(\pi)+1$, we have $\pi(i)<\pi(i+1)$. Let $r:=\pi(i)$ and $t:=\pi(i+1)$. Let $A:=\left\{\{\sigma,(r, t) \circ \sigma\}: \sigma \in S_{n}\right.$ and $\left.\sigma^{-1}(r)<\sigma^{-1}(t)\right\}$. Clearly, $A$ is a partition of $S_{n}$. Then we construct the coupling $(X, Y)$ as follows: 
(1) Choose a set in $A$ according to measure $\mu_{n, q}$, i. e. the set $\{\sigma,(r, t) \circ \sigma\}$ is chosen with probability $\mu_{n, q}(\{\sigma,(r, t) \circ \sigma\})$.

(2) Suppose the set $\{\sigma,(r, t) \circ \sigma\}$, with $\sigma^{-1}(r)<\sigma^{-1}(t)$, is chosen in the first step. Flip a coin with probability of heads being

$$
p=\frac{q^{l(\sigma)}-q^{l((r, t) \circ \sigma)}}{q^{l(\sigma)}+q^{l((r, t) \circ \sigma)}} .
$$

(3) If the outcome is head, then we set $X=Y=\sigma$.

(4) If the outcome is tail, then, with equal probability, we set either $X=\sigma, Y=$ $(r, t) \circ \sigma$ or $X=(r, t) \circ \sigma, Y=\sigma$.

Here, in the second step, the probability of head $p$ is nonnegative because $0<q \leq 1$ and the following fact:

$$
i<j \text { and } \sigma^{-1}(i)<\sigma^{-1}(j) \Rightarrow l(\sigma)<l((i, j) \circ \sigma), \quad \forall \sigma \in S_{n}
$$

It can be verified that $(X, Y)$ thus defined has the correct marginal distribution $\mu_{n, q}$. In the following we show that

$$
\operatorname{LIS}\left(\pi^{-1} \circ X\right) \geq \operatorname{LIS}\left(\tau^{-1} \circ Y\right)
$$

Then, the lemma follows from the following facts. Let $i d$ denote the identity in $S_{n}$, we have

$$
\begin{aligned}
& \operatorname{LIS}\left(\pi^{-1} \circ X\right)=\operatorname{LCS}\left(\pi^{-1} \circ X, i d\right)=\operatorname{LCS}(X, \pi), \\
& \operatorname{LIS}\left(\tau^{-1} \circ Y\right)=\operatorname{LCS}\left(\tau^{-1} \circ Y, i d\right)=\operatorname{LCS}(Y, \tau) .
\end{aligned}
$$

Here we use the facts that $\operatorname{LIS}(\pi)=\operatorname{LCS}(\pi, i d)$ for any $\pi \in S_{n}$ and

$$
\operatorname{LCS}(\pi, \tau)=\operatorname{LCS}(\sigma \circ \pi, \sigma \circ \tau)
$$

for any $\sigma, \pi, \tau \in S_{n}$. To prove (3.81), suppose the set $\{\sigma,(r, t) \circ \sigma\}$, with $\sigma^{-1}(r)<\sigma^{-1}(t)$, is chosen in the first step. If the outcome in the second step is tail, we verify that $\pi^{-1} \circ X=\tau^{-1} \circ Y$. Specifically, when $X=\sigma, Y=(r, t) \circ \sigma$, we have

$$
\pi^{-1} \circ X=\pi^{-1} \circ \sigma,
$$




$$
\tau^{-1} \circ Y=\pi^{-1} \circ(r, t) \circ(r, t) \circ \sigma=\pi^{-1} \circ \sigma
$$

When $X=(r, t) \circ \sigma, Y=\sigma$, we have

$$
\begin{gathered}
\pi^{-1} \circ X=\pi^{-1} \circ(r, t) \circ \sigma, \\
\tau^{-1} \circ Y=\pi^{-1} \circ(r, t) \circ \sigma .
\end{gathered}
$$

On the other hand, if the outcome in the second step is head, we have

$$
\begin{aligned}
& \pi^{-1} \circ X=\pi^{-1} \circ \sigma, \\
& \tau^{-1} \circ Y=\pi^{-1} \circ(r, t) \circ \sigma=s_{i} \circ \pi^{-1} \circ \sigma .
\end{aligned}
$$

Then, since

$$
\begin{aligned}
& \left(\pi^{-1} \circ \sigma\right)^{-1}(i)=\sigma^{-1}(\pi(i))=\sigma^{-1}(r), \\
& \left(\pi^{-1} \circ \sigma\right)^{-1}(i+1)=\sigma^{-1}(\pi(i+1))=\sigma^{-1}(t),
\end{aligned}
$$

the fact $\sigma^{-1}(r)<\sigma^{-1}(t)$ implies that $\tau^{-1} \circ Y$ covers $\pi^{-1} \circ X$ in $\left(S_{n}, \leq_{L}\right)$. Hence, we have (3.81).

Lemma 3.4.6 can be proved in a similar argument and we omit its proof. We complete this section by proving Lemma 3.4.1 and Lemma 3.4.2 using Lemma 3.4.5. Lemma 3.4.3 and Lemma 3.4.4 follow from Lemma 3.4.6 in the same way.

Proof of Lemma 3.4.1. By Lemma 3.2.6, we have $\mu_{n, q_{1}} \preceq \mu_{n, q_{2}}$ with the underlying partial order being the right weak Bruhat order. By Strassen's theorem [27], there exists a coupling $\left(Y_{1}, Y_{2}\right)$ such that $Y_{1} \sim \mu_{n, q_{1}}, Y_{2} \sim \mu_{n, q_{2}}$ and $Y_{1} \leq_{R} Y_{2}$. Next, for any $(\pi, \tau)$ with $\pi \leq_{R} \tau$, let $\left(X_{\pi, \tau}, Y_{\pi, \tau}\right)$ be the coupling constructed in Lemma 3.4.5. Then we define $\left(X_{1}, X_{2}\right)$ as follows. Conditioned on $\left(Y_{1}, Y_{2}\right)=(\pi, \tau)$, define $X_{1}=X_{\pi, \tau}$, $X_{2}=Y_{\pi, \tau}$. We show that $\left(X_{1}, X_{2}, Y_{1}, Y_{2}\right)$ thus defined satisfies all three requirements. Firstly, since both marginal distributions of $\left(X_{\pi, \tau}, Y_{\pi, \tau}\right)$ are $\mu_{n, q}$ for any $(\pi, \tau)$ with $\pi \leq_{R} \tau$, the $X_{1}, X_{2}$ defined above both have distribution $\mu_{n, q}$. Next, note that no matter what value $\left(Y_{1}, Y_{2}\right)$ takes, the conditional distribution of $X_{i}$ is always $\mu_{n, q}$. 
Hence $X_{i}$ is independent of $\left(Y_{1}, Y_{2}\right)$. Finally, $\operatorname{LCS}\left(X_{1}, Y_{1}\right) \geq \operatorname{LCS}\left(X_{2}, Y_{2}\right)$ follows by the construction of the coupling and Lemma 3.4.5.

Proof of Lemma 3.4.2. Since the identity $i d$ is the minimum of $\left(S_{n}, \leq_{R}\right)$, for any $\tau \in$ $S_{n}$, define $\left(X_{\tau}, Y_{\tau}\right)$ to be the coupling constructed in Lemma 3.4 .5 with $\pi$ being the identity $i d$. Then, we define the coupling $(X, Y, Z)$ as follows. First sample $Z$ according to $\mu_{n, q^{\prime}}$. Then, conditioned on $Z=\tau$, define $X=X_{\tau}, Y=Y_{\tau}$. Since $X_{\tau}$ and $Y_{\tau}$ are both $\mu_{n, q}$-distributed for any $\tau \in S_{n}, X$ and $Y$ thus defined are both $\mu_{n, q}$-distributed. By the definition of $Y$, the distribution of $Y$ is independent of the choice of $\tau$. Hence $Y$ and $Z$ are independent. Finally, by Lemma 3.4.5, we have $\operatorname{LCS}(Y, Z) \leq \operatorname{LCS}(X, i d)=$ $\operatorname{LIS}(X)$.

\subsection{Proof of WLLN when only $\beta$ is finite}

Given $\gamma>0$ and two sequences $\left\{q_{n}\right\}_{n=1}^{\infty},\left\{q_{n}^{\prime}\right\}_{n=1}^{\infty}$ such that $\lim _{n \rightarrow \infty} n(1-$ $\left.q_{n}\right)=\infty$, and $\lim _{n \rightarrow \infty} n\left(1-q_{n}^{\prime}\right)=\beta$, define a new sequence $\left\{\bar{q}_{n}\right\}_{n=1}^{\infty}$ by setting

$\bar{q}_{n}=\max \left(q_{n}, 1-\frac{\gamma}{n}\right)$. Note that, for $n$ sufficiently large, we have $q_{n}<1-\frac{\gamma}{n}$. Hence $\lim _{n \rightarrow \infty} n\left(1-\bar{q}_{n}\right)=\gamma$.

Assuming $\beta>0$, by Lemma 3.4.1 and Lemma 3.4.2, for sufficiently large $n$, we can construct a coupling $\left(X_{n}, Y_{n}, Z_{n}, X_{n}^{\prime}, Y_{n}^{\prime}\right)$ such that

- $X_{n} \sim \mu_{n, q_{n}}, Y_{n} \sim \mu_{n, q_{n}^{\prime}}, Z_{n} \sim \mu_{n, q_{n}^{\prime}}, X_{n}^{\prime} \sim \mu_{n, \bar{q}_{n}}$ and $Y_{n}^{\prime} \sim \mu_{n, q_{n}^{\prime}}$,

- $X_{n}$ and $Y_{n}$ are independent. $X_{n}^{\prime}$ and $Y_{n}^{\prime}$ are independent.

$$
\operatorname{LCS}\left(X_{n}^{\prime}, Y_{n}^{\prime}\right) \leq \operatorname{LCS}\left(X_{n}, Y_{n}\right) \leq \operatorname{LIS}\left(Z_{n}\right)
$$

By Theorem 1.1.7,

$$
\frac{\operatorname{LIS}\left(Z_{n}\right)}{\sqrt{n}} \stackrel{p}{\longrightarrow} 2 \kappa(\beta) .
$$

On the other hand, by Theorem 3,

$$
\frac{\operatorname{LCS}\left(X_{n}^{\prime}, Y_{n}^{\prime}\right)}{\sqrt{n}} \stackrel{p}{\longrightarrow} 2 \bar{J}_{\beta, \gamma}
$$


where $\bar{J}_{\beta, \gamma}=\sup _{\phi \in B_{\nearrow}^{1}} J\left(\phi, \rho_{\beta, \gamma}\right)$. Here $B_{\nearrow}^{1}$ denotes the set of nondecreasing, $C_{b}^{1}$ functions $\phi:[0,1] \rightarrow[0,1]$, with $\phi(0)=0$ and $\phi(1)=1$ and

$$
\begin{aligned}
\rho_{\beta, \gamma}(x, y) & :=\int_{0}^{1} u_{\beta}(x, t) u_{\gamma}(t, y) d t \\
u_{\beta}(x, y) & :=\frac{(\beta / 2) \sinh (\beta / 2)}{\left(e^{\beta / 4} \cosh (\beta[x-y] / 2)-e^{-\beta / 4} \cosh (\beta[x+y-1] / 2)\right)^{2}} \\
\text { when } \beta & \neq 0, \text { and } u_{0}(x, y):=1, \\
J\left(\phi, \rho_{\beta, \gamma}\right) & :=\int_{0}^{1} \sqrt{\dot{\phi}(x) \rho_{\beta, \gamma}(x, \phi(x))} d x .
\end{aligned}
$$

By (3.85), (3.86) and (3.87), to prove Theorem 4, it suffices to show

$$
\lim _{\gamma \rightarrow \infty} \bar{J}_{\beta, \gamma}=\kappa(\beta)
$$

For the case $\beta<0$, the same argument applies except that we construct the coupling $\left(X_{n}, Y_{n}, Z_{n}, X_{n}^{\prime}, Y_{n}^{\prime}\right)$ based on Lemma 3.4.3 and Lemma 3.4.4 such that

$$
\operatorname{LIS}\left(Z_{n}\right) \leq \operatorname{LCS}\left(X_{n}, Y_{n}\right) \leq \operatorname{LCS}\left(X_{n}^{\prime}, Y_{n}^{\prime}\right)
$$

For the case $\beta=0$, we can split $\left\{q_{n}^{\prime}\right\}$ into two subsequences with one has those $q_{n}^{\prime} \leq 1$ and the other has those $q_{n}^{\prime}>1$. The argument for the case when $\beta>0$ applies for the first subsequence and the argument for the case when $\beta<0$ applies for the second subsequence.

To show $(3.91)$, we will prove that $\rho_{\beta, \gamma}(x, y)$ converges uniformly to $u_{\beta}(x, y)$ on $[0,1] \times[0,1]$ as $\gamma$ goes to infinity, and the following lemma is a key step.

Lemma 3.5.1. For any $\epsilon>0$, there exists $N$ such that, when $\gamma>N$, for any $x, y \in$ $[0,1]$ with $|x-y|>\epsilon$, we have $u_{\gamma}(x, y)<\epsilon$.

Proof. By (3.89), we have $u_{\gamma}(x, y)=u_{\gamma}(y, x)$. Hence, without loss of generality, we assume $x>y$. Given $\gamma>0$, we have

$$
\begin{aligned}
u_{\gamma}(x, y) & =\frac{(\gamma / 2) \sinh (\gamma / 2)}{\left(e^{\gamma / 4} \cosh (\gamma[x-y] / 2)-e^{-\gamma / 4} \cosh (\gamma[x+y-1] / 2)\right)^{2}} \\
& =\frac{\gamma\left(1-e^{-\gamma}\right)}{4\left(\cosh (\gamma[x-y] / 2)-e^{-\gamma / 2} \cosh (\gamma[x+y-1] / 2)\right)^{2}}
\end{aligned}
$$




$$
\begin{aligned}
& =\frac{\gamma\left(1-e^{-\gamma}\right)}{\left(e^{\gamma(x-y) / 2}+e^{\gamma(y-x) / 2}-e^{\gamma(x+y-2) / 2}-e^{-\gamma(x+y) / 2}\right)^{2}} \\
& \leq \frac{\gamma}{\left(e^{\gamma|x-y| / 2}-1\right)^{2}} .
\end{aligned}
$$

Here the last inequality (3.94) follows, because, considering the term inside the square in the denominator of (3.93), we have

$$
\begin{aligned}
& e^{\gamma(x-y) / 2}+e^{\gamma(y-x) / 2}-e^{\gamma(x+y-2) / 2}-e^{-\gamma(x+y) / 2} \\
= & \left(e^{\gamma(x-y) / 2}-1\right)+\left(1-e^{\gamma(x+y-2) / 2}\right)+\left(e^{\gamma(y-x) / 2}-e^{-\gamma(x+y) / 2}\right) .
\end{aligned}
$$

Each term inside the three parentheses in (3.95) are non negative. Then, since $|x-y|>$ $\epsilon$, by (3.94), we have

$$
u_{\gamma}(x, y) \leq \frac{\gamma}{\left(e^{\gamma \epsilon / 2}-1\right)^{2}}
$$

We can choose $N$ such that for any $\gamma>N, \frac{\gamma}{\left(e^{\gamma \epsilon / 2}-1\right)^{2}}<\epsilon$.

The following two facts about the density $u_{\beta}(x, y)$, which are proved in Lemma 2.1.9 and Lemma 3.3.5 respecively, will be used in the proof of next lemma. The first one is that $u_{\beta}(x, y)$ has uniform marginal measure.

$$
\int_{0}^{1} u_{\beta}(x, y) d y=1, \quad \forall x \in[0,1]
$$

The second one is that $u_{\beta}(x, y) \in C_{b}^{1}$. Specifically, we have

$$
e^{-|\beta|} \leq u_{\beta}(x, y) \leq e^{|\beta|}, \quad \max \left(\left|\frac{\partial u_{\beta}}{\partial x}\right|,\left|\frac{\partial u_{\beta}}{\partial y}\right|\right) \leq|\beta| e^{|\beta|} .
$$

\section{Lemma 3.5.2.}

$$
\lim _{\gamma \rightarrow+\infty} \sup _{x, y \in[0,1]}\left|\rho_{\beta, \gamma}(x, y)-u_{\beta}(x, y)\right|=0 .
$$

Proof. Given $\epsilon>0$, by Lemma 3.5.1, we can choose $N>0$ such that, for any $\gamma>N$, we have $u_{\gamma}(x, y)<\epsilon$ whenever $|x-y|>\epsilon$. Define $A_{y, \epsilon}:=[0,1] \backslash[y-\epsilon, y+\epsilon]$. Assuming that $u_{\beta}, u_{\gamma}$ and $\rho_{\beta, \gamma}$ are all zero outside of the unit square $[0,1] \times[0,1]$, for any $\gamma>N$, we have

$$
\left|\rho_{\beta, \gamma}(x, y)-\int_{y-\epsilon}^{y+\epsilon} u_{\beta}(x, t) u_{\gamma}(t, y) d t\right|
$$




$$
\begin{aligned}
& =\left|\int_{0}^{1} u_{\beta}(x, t) u_{\gamma}(t, y) d t-\int_{y-\epsilon}^{y+\epsilon} u_{\beta}(x, t) u_{\gamma}(t, y) d t\right| \\
& =\int_{A_{y, \epsilon}} u_{\beta}(x, t) u_{\gamma}(t, y) d t \\
& \leq \epsilon \int_{A_{y, \epsilon}} u_{\beta}(x, t) d t \\
& \leq \epsilon .
\end{aligned}
$$

Here the last inequality follows from (3.97). By (3.98), for any $t \in[y-\epsilon, y+\epsilon]$, we have

$$
\left|u_{\beta}(x, y)-u_{\beta}(x, t)\right| \leq \epsilon|\beta| e^{|\beta|} .
$$

By (3.97) and Lemma 3.5.1, we have

$$
1 \geq \int_{y-\epsilon}^{y+\epsilon} u_{\gamma}(t, y) d t=1-\int_{A_{y, \epsilon}} u_{\gamma}(t, y) d t>1-\epsilon .
$$

Hence, we have

$$
\begin{aligned}
& \int_{y-\epsilon}^{y+\epsilon} u_{\beta}(x, t) u_{\gamma}(t, y) d t \\
\leq & \left(u_{\beta}(x, y)+\epsilon|\beta| e^{|\beta|}\right) \int_{y-\epsilon}^{y+\epsilon} u_{\gamma}(t, y) d t \\
\leq & u_{\beta}(x, y)+\epsilon|\beta| e^{|\beta|},
\end{aligned}
$$

and

$$
\begin{aligned}
& \int_{y-\epsilon}^{y+\epsilon} u_{\beta}(x, t) u_{\gamma}(t, y) d t \\
\geq & \left(u_{\beta}(x, y)-\epsilon|\beta| e^{|\beta|}\right) \int_{y-\epsilon}^{y+\epsilon} u_{\gamma}(t, y) d t \\
\geq & \left(u_{\beta}(x, y)-\epsilon|\beta| e^{|\beta|}\right)(1-\epsilon) \\
\geq & u_{\beta}(x, y)-\epsilon\left(e^{|\beta|}+|\beta| e^{|\beta|}\right) .
\end{aligned}
$$

Here we use the fact that $u_{\beta}(x, y) \leq e^{|\beta|}$ in the last inequality. By combining (3.100), (3.101) and (3.102), it follows that, for any $\gamma>N$ and any $x, y \in[0,1]$,

$$
\left|\rho_{\beta, \gamma}(x, y)-u_{\beta}(x, y)\right| \leq \epsilon\left(1+e^{|\beta|}+|\beta| e^{|\beta|}\right)
$$


Now we are in the position to show (3.91) which completes the proof of Theorem 4.

Proof of Theorem 4. In [22], Mueller and Starr show that

$$
\kappa(\beta)=\sup _{\phi \in B_{\nearrow}^{1}} J\left(\phi, u_{\beta}\right)=\sup _{\phi \in B_{\nearrow}^{1}} \int_{0}^{1} \sqrt{\dot{\phi}(x) u_{\beta}(x, \phi(x))} d x .
$$

In fact, they show that the supremum is attained when $\phi(x)=x$, i.e.

$$
\kappa(\beta)=\int_{0}^{1} \sqrt{u_{\beta}(x, x)} d x
$$

We claim that, to show (3.91), it suffices to show that

$$
\lim _{\gamma \rightarrow \infty} \sup _{\phi \in B_{\gamma}^{1}}\left|J\left(\phi, \rho_{\beta, \gamma}\right)-J\left(\phi, u_{\beta}\right)\right|=0
$$

To see this, let $g$ denote the identity function, i.e., $g(x)=x$. Since $\bar{J}_{\beta, \gamma} \geq J\left(g, \rho_{\beta, \gamma}\right)$, we have

$$
\liminf _{\gamma \rightarrow \infty} \bar{J}_{\beta, \gamma} \geq \liminf _{\gamma \rightarrow \infty} J\left(g, \rho_{\beta, \gamma}\right)=J\left(g, u_{\beta}\right)=\kappa(\beta) .
$$

Here we use the fact follows by (3.105) that

$$
\lim _{\gamma \rightarrow \infty}\left|J\left(g, \rho_{\beta, \gamma}\right)-J\left(g, u_{\beta}\right)\right|=0
$$

On the other hand, choose $\left\{\gamma_{n}\right\}$ and $\left\{\phi_{n}\right\}$ such that $\gamma_{n} \rightarrow \infty, \phi_{n} \in B_{\nearrow}^{1}$ and

$$
\lim _{n \rightarrow \infty} J\left(\phi_{n}, \rho_{\beta, \gamma_{n}}\right)=\limsup _{\gamma \rightarrow \infty} \bar{J}_{\beta, \gamma}
$$

By (3.105), we have

$$
\lim _{n \rightarrow \infty} J\left(\phi_{n}, \rho_{\beta, \gamma_{n}}\right)=\lim _{n \rightarrow \infty} J\left(\phi_{n}, u_{\beta}\right) \leq \kappa(\beta)
$$

The claim follows from (3.106), (3.107) and (3.108).

Given $0<\epsilon<\frac{e^{-|\beta|}}{2}$, by Lemma 3.5.2, there exists $N$ such that, for any $\gamma>N$ and any $x, y \in[0,1]$, we have $\left|\rho_{\beta, \gamma}(x, y)-u_{\beta}(x, y)\right|<\epsilon$. Hence, by (3.98), we have

$$
\rho_{\beta, \gamma}(x, y) \geq u_{\beta}(x, y)-\epsilon \geq \frac{e^{-|\beta|}}{2} .
$$


Therefore, when $\gamma>N$, for any $\phi \in B_{\gamma}^{1}$, we have

$$
\begin{aligned}
& \left|J\left(\phi, \rho_{\beta, \gamma}\right)-J\left(\phi, u_{\beta}\right)\right| \\
\leq & \int_{0}^{1}\left|\sqrt{\dot{\phi}(x) \rho_{\beta, \gamma}(x, \phi(x))}-\sqrt{\dot{\phi}(x) u_{\beta}(x, \phi(x))}\right| d x \\
= & \int_{0}^{1} \frac{\dot{\phi}(x)\left|\rho_{\beta, \gamma}(x, \phi(x))-u_{\beta}(x, \phi(x))\right|}{\sqrt{\dot{\phi}(x) \rho_{\beta, \gamma}(x, \phi(x))}+\sqrt{\dot{\phi}(x) u_{\beta}(x, \phi(x))}} d x \\
\leq & \frac{\epsilon e^{|\beta| / 2}}{\sqrt{2}} \int_{0}^{1} \sqrt{\dot{\phi}(x)} d x \leq \frac{\epsilon e^{|\beta| / 2}}{\sqrt{2}}\left(\int_{0}^{1} \dot{\phi}(x) d x\right)^{1 / 2} \\
= & \frac{\epsilon e^{|\beta| / 2}}{\sqrt{2}} .
\end{aligned}
$$

Here the last inequality follows by Cauchy-Schwarz inequality. (3.105) follows from $(3.110)$.

\subsection{Proof of Lemma 3.3.1 and Lemma 3.3.2}

We first introduce two corollaries of these two lemmas. Recall that, for any positive integer $n$ and $m \in[n]$, we define

$$
Q(n, m):=\left\{\left(b_{1}, b_{2}, \ldots, b_{m}\right): b_{i} \in[n] \text { and } b_{i}<b_{i+1} \text { for all } i\right\}
$$

By choosing $k_{n}=n$ in these two lemmas and the fact that $Q(n, n)$ contains a single member $\{(1,2, \ldots, n)\}$, we can recover Corollary 4.3 of [22]:

Corollary 3.6.1. Suppose that $\left\{q_{n}\right\}_{n=1}^{\infty}$ is a sequence such that $q_{n} \geq 1$ and $\liminf _{n \rightarrow \infty} n\left(1-q_{n}\right)=\beta$, with $\beta \in \mathbb{R}$. We have

$$
\lim _{n \rightarrow \infty} \mu_{n, q_{n}}\left(\pi \in S_{n}: \frac{\operatorname{LIS}(\pi)}{\sqrt{n}} \notin\left(2 e^{\frac{\beta}{2}}-\epsilon, 2+\epsilon\right)\right)=0
$$

for any $\epsilon>0$.

Corollary 3.6.2. Suppose that $\left\{q_{n}\right\}_{n=1}^{\infty}$ is a sequence such that $0<q_{n} \leq 1$ and $\lim \sup _{n \rightarrow \infty} n\left(1-q_{n}\right)=\beta<\ln 2$. We have

$$
\lim _{n \rightarrow \infty} \mu_{n, q_{n}}\left(\pi \in S_{n}: \frac{L I S(\pi)}{\sqrt{n}} \notin\left(2-\epsilon, 2 e^{\frac{\beta}{2}}+\epsilon\right)\right)=0,
$$

for any $\epsilon>0$. 
To prove these two lemmas, we use the same techniques developed in the proof of Corollary 4.3 in [22], in which they constructed a coupling of two point processes. A point process is a random, locally finite, nonnegative integer valued measure. Let $\mathcal{X}_{k}$ denote the set of all Borel measures $\xi$ on $\mathbb{R}^{k}$ such that $\xi(A) \in\{0,1,2, \ldots\}$ for any bounded Borel set $A$ in $\mathbb{R}^{k}$. Then, a point process on $\mathbb{R}^{k}$ is a random variable which takes value in $\mathcal{X}_{k}$.

Suppose $\mu, \nu$ are two measures on $\mathbb{R}^{k}$. We say $\mu \leq \nu$ if $\mu(A) \leq \nu(A)$ for any $A \in \mathcal{B}\left(\mathbb{R}^{k}\right)$.

Lemma 3.6.3. Suppose $\hat{\alpha}$ and $\alpha$ are two measures on $[0,1]$ with density $f(x), g(x)$ respectively. If, for any $x \in[0,1], f(x) \geq p \cdot g(x)$ for some $1>p>0$, then there exist random variables $X, Y$ and $B_{p}$ such that the following hold.

- $X$ is $\hat{\alpha}$-distributed, $Y$ is $\alpha$-distributed and $B_{p}$ is Bernoulli distributed with $\mathbb{P}\left(B_{p}=1\right)=p$.

- $B_{p}$ and $Y$ are independent.

- Define two point processes $\eta, \xi$ on $[0,1]$ as follows,

$$
\xi(A):=\mathbb{1}_{A}(X) \quad \text { and } \quad \eta(A):=B_{p} \cdot \mathbb{1}_{A}(Y), \quad \forall A \in \mathcal{B}([0,1])
$$

Then, we have $\eta \leq \xi$ almost surely.

Proof. Let $Y, Y^{\prime}$ and $B_{p}$ be independent random variables defined on the same probability space such that $Y$ is $\alpha$-distributed, $B_{p}$ is Bernoulli distributed with $\mathbb{P}\left(B_{p}=1\right)=p$ and the density of the distribution of $Y^{\prime}$ is $\frac{f(x)-p \cdot g(x)}{1-p}$. Define $X=B_{p} Y+\left(1-B_{p}\right) Y^{\prime}$. It can be easily verified that $X$ thus defined is $\hat{\alpha}$-distributed. Because,

$$
\mathbb{P}(X \in A)=p \int_{A} g(x) d x+(1-p) \int_{A} \frac{f(x)-p \cdot g(x)}{1-p} d x=\int_{A} f(x) d x,
$$

for any $A \in \mathcal{B}([0,1])$. Finally, the two point processes $\xi$ and $\eta$ thus defined satisfy $\eta \leq \xi$, since for any $A \in \mathcal{B}([0,1])$, when $B_{p}=1$, we have $\xi(A)=\eta(A)$, and, when $B_{p}=0$, we have $\eta(A)=0$. 
Lemma 3.6.4. Suppose $\hat{\alpha}$ and $\alpha$ are two measures on $[0,1]$ with density $f(x), g(x)$ respectively. If, for any $x \in[0,1],\left(1-\theta_{1}\right) g(x) \leq f(x) \leq\left(1+\theta_{2}\right) g(x)$ for some $\theta_{1}, \theta_{2} \geq 0$ with $\theta_{1}+\theta_{2}<1$, then there exist random variables $X, Y, Z$ and $B_{\theta}$ such that the following hold.

- $X$ is $\hat{\alpha}$-distributed, $Y$ and $Z$ are $\alpha$-distributed and $B_{\theta}$ is Bernoulli distributed with $\mathbb{P}\left(B_{\theta}=1\right)=\theta$, where $\theta=\theta_{1}+\theta_{2}$.

- $B_{\theta}, Y$ and $Z$ are independent.

- Define two point processes $\xi, \zeta$ on $[0,1]$ as follows,

$$
\xi(A):=\mathbb{1}_{A}(X) \quad \text { and } \quad \zeta(A):=\mathbb{1}_{A}(Y)+B_{\theta} \cdot \mathbb{1}_{A}(Z), \quad \forall A \in \mathcal{B}([0,1]) .
$$

Then, we have $\xi \leq \zeta$ almost surely.

Proof. Let $Y, Z$ and $B_{\theta}$ be independent random variables defined on the same probability space such that $Y, Z$ is $\alpha$-distributed, $B_{\theta}$ is Bernoulli distributed with $\mathbb{P}\left(B_{\theta}=\right.$ $1)=\theta$. We define a new random variable $X$ as follows. Conditioned on $Y=y$ and $Z=z$,

- if $B_{\theta}=0$, define $X=y$

- if $B_{\theta}=1$, we flip a coin $W$ with probability of head being $\frac{f(z)-\left(1-\theta_{1}\right) g(z)}{\theta \cdot g(z)}$. If the result is head, define $X=z$. Else, define $X=y$.

Note that, without loss of generality, here we may assume $g(z)>0$, since $\mathbb{P}(g(Z)=0)=0$. It is straight forward that the two point processes $\xi$ and $\zeta$ thus defined satisfy $\xi \leq \zeta$ a.s.. We complete the proof by verifying that $X$ thus defined has distribution $f(x)$.

For any $A \in \mathcal{B}([0,1])$, the event $\{X \in A\}$ can be partitioned into three parts:

$\left\{B_{\theta}=0, Y \in A\right\},\left\{B_{\theta}=1, W\right.$ is head,$\left.Z \in A\right\}$ and $\left\{B_{\theta}=1, W\right.$ is tail,$\left.Y \in A\right\}$. We have

$$
\mathbb{P}\left(\left\{B_{\theta}=0, Y \in A\right\}\right)=(1-\theta) \int_{A} g(x) d x=(1-\theta) \alpha(A),
$$




$$
\begin{aligned}
\mathbb{P}\left(\left\{B_{\theta}=1, W \text { is head }, Z \in A\right\}\right) & =\theta \int_{A} \frac{f(z)-\left(1-\theta_{1}\right) g(z)}{\theta \cdot g(z)} g(z) d z \\
& =\int_{A} f(z) d z-\left(1-\theta_{1}\right) \alpha(A), \\
\mathbb{P}\left(\left\{B_{\theta}=1, W \text { is tail }, Y \in A\right\}\right) & =\theta \int_{A} g(y) d y \int_{0}^{1}\left(1-\frac{f(z)-\left(1-\theta_{1}\right) g(z)}{\theta \cdot g(z)}\right) g(z) d z \\
& =\alpha(A) \int_{0}^{1}\left(1+\theta_{2}\right) g(z)-f(z) d z \\
& =\alpha(A) \theta_{2} .
\end{aligned}
$$

Here we evaluate the last two probabilities by conditioning on the value of $Z$. Summing up the three probabilities, we get

$$
\mathbb{P}(\{X \in A\})=\int_{A} f(z) d z .
$$

Next, we define a triangular array of random variables in $[0,1]$.

Definition 3.6.5. Suppose that $\left\{q_{n}\right\}_{n=1}^{\infty}$ is a sequence such that $q_{n}>0$. For any $n \in \mathbb{N}$, we define the random vector $\left(Y_{1}^{(n)}, Y_{2}^{(n)}, \ldots, Y_{n}^{(n)}\right)$ as follows. Let $\left\{Y_{i}\right\}_{i=1}^{n}$ be i.i.d. uniform random variables on $[0,1]$. Let $\left\{Y_{(i)}\right\}_{i=1}^{n}$ be the order statistics of $\left\{Y_{i}\right\}_{i=1}^{n}$. Independently, let $\pi$ be a $\mu_{n, q_{n}}$-distributed random variable on $S_{n}$. We define $Y_{i}^{(n)}:=Y_{(\pi(i))}$ for all $i \in[n]$.

In the remainder of this paper, we use $\left(Y_{1}^{(n)}, Y_{2}^{(n)}, \ldots, Y_{n}^{(n)}\right)$ specifically to denote the random vector defined as above. Also, we define the function $\Phi$ which maps vectors in $\mathbb{R}^{n}$ or $n$ points in $\mathbb{R}^{2}$ to the induced permutation in $S_{n}$.

Definition 3.6.6. Suppose $\boldsymbol{x}=\left(x_{1}, x_{2}, \ldots, x_{n}\right)$ is a vector in $\mathbb{R}^{n}$ such that all its entries are different. Let $\Phi(\boldsymbol{x})$ denote the permutation in $S_{n}$ such that, for any $i \in[n]$, $\Phi(\boldsymbol{x})(i)=j$ if $x_{i}$ is the $j$-th smallest entry in $\boldsymbol{x}$. Similarly, suppose $\boldsymbol{z}=\left\{\left(x_{i}, y_{i}\right)\right\}_{i=1}^{n}$ are $n$ points in $\mathbb{R}^{2}$ such that they share no $x$ coordinate nor any $y$ coordinate. Let $\Phi(\boldsymbol{x})$ denote the permutation in $S_{n}$ such that, for any $i \in[n], \Phi(\boldsymbol{z})(i)=j$ if there exits $k \in[n]$, such that $x_{k}$ is the $i$-th smallest term in $\left\{x_{i}\right\}_{i=1}^{n}$ and $y_{k}$ is the $j$-th smallest term in $\left\{y_{i}\right\}_{i=1}^{n}$. 
Remark. From the above definitions, it can be easily seen that

(a) For any $x_{1}<x_{2}<\cdots<x_{n}$ and $\boldsymbol{y}=\left(y_{1}, y_{2}, \ldots, y_{n}\right) \in \mathbb{R}^{n}$, we have $\Phi(\boldsymbol{y})=$ $\Phi\left(\left\{\left(x_{i}, y_{i}\right)\right\}_{i=1}^{n}\right)$.

(b) For any $\boldsymbol{y}=\left(y_{1}, y_{2}, \ldots, y_{n}\right) \in \mathbb{R}^{n}$ and $\boldsymbol{b}=\left(b_{1}, b_{2}, \ldots, b_{m}\right) \in Q(n, m)$, we have $\Phi(\boldsymbol{y})_{\boldsymbol{b}}=\Phi\left(\left(y_{b_{1}}, y_{b_{2}}, \ldots, y_{b_{m}}\right)\right)$.

(c) $\Phi\left(\left(Y_{1}^{(n)}, Y_{2}^{(n)}, \ldots, Y_{n}^{(n)}\right)\right)$ is $\mu_{n, q_{n}}$-distributed.

It is not hard to show that the density function of $\left(Y_{1}^{(n)}, Y_{2}^{(n)}, \ldots, Y_{n}^{(n)}\right)$ is the following (in the sense of a.s.),

$$
f_{n}(\boldsymbol{y})=\mu_{n, q_{n}}(\Phi(\boldsymbol{y})) \cdot n ! \quad \text { for all } \boldsymbol{y} \in[0,1]^{n} \backslash \text { Diagonal } .
$$

Here the set Diagonal consists of all those vectors which contain (at least two) identical entries. Since $\left\{Y_{i}^{(n)}\right\}_{i=1}^{n}=\left\{Y_{i}\right\}_{i=1}^{n}$ are $n$ i.i.d. uniform samples from $[0,1]$, we have $\mathbb{P}\left(\left(Y_{1}^{(n)}, Y_{2}^{(n)}, \ldots, Y_{n}^{(n)}\right) \in\right.$ Diagonal $)=0$. Intuitively, for any $0 \leq y_{1}<\cdots<y_{n} \leq$ 1 , there are $n$ ! ways to choose the vector $\left(Y_{1}, \ldots, Y_{n}\right)$ such that $\left\{Y_{i}\right\}_{i=1}^{n}=\left\{y_{i}\right\}_{i=1}^{n}$. Conditioned on $\left\{Y_{i}\right\}_{i=1}^{n}=\left\{y_{i}\right\}_{i=1}^{n}$, the probability of $\left(Y_{1}^{(n)}, \ldots, Y_{n}^{(n)}\right)=\left(y_{\pi(1)}, \ldots, y_{\pi(n)}\right)$ is $\mu_{n, q_{n}}(\pi)$.

Lemma 3.6.7. Given $i \in[n]$ and a vector $\left(y_{1}, \ldots, y_{i-1}, y_{i+1}, \ldots, y_{n}\right) \in[0,1]^{n-1} \backslash$ Diagonal, let $\hat{\alpha}$ denote the distribution of $Y_{i}^{(n)}$ conditioned on the event $\left\{Y_{j}^{(n)}=\right.$ $y_{j}$ for all $\left.j \in[n] \backslash\{i\}\right\}$. Then $\hat{\alpha}$ has density $f(y)$ on $[0,1]$ such that, excluding a set $G$ of measure zero, for any $y, y^{\prime} \in[0,1] \backslash G$, we have

$$
f(y) \geq \min \left(q_{n}^{n}, \frac{1}{q_{n}^{n}}\right), \quad f(y)-f\left(y^{\prime}\right) \leq \max \left(q_{n}^{n}, \frac{1}{q_{n}^{n}}\right)-1 .
$$

Proof. Since $\left(Y_{1}^{(n)}, Y_{2}^{(n)}, \ldots, Y_{n}^{(n)}\right)$ has density $f_{n}(\boldsymbol{y})=\mu_{n, q_{n}}(\Phi(\boldsymbol{y})) \cdot n !$ on $[0,1]^{n} \backslash$ Diagonal, the density $f(y)$ of $\hat{\alpha}$ is given by

$$
f(y)=\frac{\mu_{n, q_{n}}\left(\Phi\left(\left(y_{1}, y_{2}, \ldots, y_{i-1}, y, y_{i+1}, \ldots, y_{n}\right)\right)\right)}{\int_{0}^{1} \mu_{n, q_{n}}\left(\Phi\left(\left(y_{1}, y_{2}, \ldots, y_{i-1}, t, y_{i+1}, \ldots, y_{n}\right)\right)\right) d t},
$$


for any $y \in[0,1] /\left\{y_{1}, y_{2}, \ldots, y_{i-1}, y_{i+1}, \ldots, y_{n}\right\}$.

It can be seen from the definition that $f(y)$ is a simple function which takes at most $n$ different values. Let $M$ and $m$ denote the maximum and minimum of $f(y)$ respectively. Then we have $M \geq 1$ and $0<m \leq 1$. Moreover, for any $y, y^{\prime} \in[0,1]$, let $\boldsymbol{y}:=$ $\left(y_{1}, y_{2}, \ldots, y_{i-1}, y, y_{i+1}, \ldots, y_{n}\right)$ and $\boldsymbol{y}^{\prime}:=\left(y_{1}, y_{2}, \ldots, y_{i-1}, y^{\prime}, y_{i+1}, \ldots, y_{n}\right)$. We have

$$
\left|l(\Phi(\boldsymbol{y}))-l\left(\Phi\left(\boldsymbol{y}^{\prime}\right)\right)\right| \leq n-1
$$

That is, if $\boldsymbol{y}$ and $\boldsymbol{y}^{\prime}$ differ at one entry, the number of inversions of the induced permutations differ at most by $n-1$. Hence, assuming $q_{n} \geq 1$, for any $y, y^{\prime} \in[0,1]$, we have

$$
\frac{1}{q_{n}^{n-1}} \leq \frac{f(y)}{f\left(y^{\prime}\right)} \leq q_{n}^{n-1}
$$

Choose $y^{\prime}$ such that $f\left(y^{\prime}\right)=M$, we have $f(y) \geq M / q_{n}^{n-1} \geq 1 / q_{n}^{n}$. For the second part, we choose $y, y^{\prime}$ such that $f(y)=M$ and $f\left(y^{\prime}\right)=m$. Then we have $M / m-1 \leq$ $q_{n}^{n-1}-1 \leq q_{n}^{n}-1$. Thus, $M-m \leq q_{n}^{n}-1$, since $0<m \leq 1$.

On the other hand, assuming $0<q_{n}<1$, by the similar argument it follows that for any $y, y^{\prime} \in[0,1]$, we have

$$
q_{n}^{n-1} \leq \frac{f(y)}{f\left(y^{\prime}\right)} \leq \frac{1}{q_{n}^{n-1}}
$$

Choose $y^{\prime}$ such that $f\left(y^{\prime}\right)=M$, we have $f(y) \geq M q_{n}^{n-1} \geq q_{n}^{n}$. For the second part, we choose $y, y^{\prime}$ such that $f(y)=M$ and $f\left(y^{\prime}\right)=m$. Then we have $M / m-1 \leq 1 / q_{n}^{n-1}-1 \leq$ $1 / q_{n}^{n}-1$. Thus, $M-m \leq 1 / q_{n}^{n}-1$, since $0<m \leq 1$.

Combining the two cases above, the lemma follows.

Lemma 3.6.8. Given $n \in \mathbb{N}$ and $q_{n} \geq 1$, for any $m \leq n$ and any $\boldsymbol{b}=\left(b_{1}, b_{2}, \ldots, b_{m}\right) \in$ $Q(n, m)$, there exists a random vector $\left(V_{1}, V_{2}, \ldots, V_{n}\right) \in[0,1]^{n}$ and $2 m$ independent random variables $\left\{U_{i}\right\}_{i=1}^{m} \cup\left\{B_{i}\right\}_{i=1}^{m}$ such that $\left(V_{1}, V_{2}, \ldots, V_{n}\right)$ has the same distribution as $\left(Y_{1}^{(n)}, Y_{2}^{(n)}, \ldots, Y_{n}^{(n)}\right)$, each $U_{i}$ is uniformly distributed on $[0,1]$ and each $B_{i}$ is a Bernoulli random variable with $\mathbb{P}\left(B_{i}=1\right)=\frac{1}{q_{n}^{n}}$. Moreover, if we define two point processes as follows,

$$
\xi_{\boldsymbol{b}}^{(n)}(A):=\sum_{i=1}^{m} \mathbb{1}_{A}\left(\left(i, V_{b_{i}}\right)\right), \quad \eta_{m}(A):=\sum_{i=1}^{m} B_{i} \cdot \mathbb{1}_{A}\left(\left(i, U_{i}\right)\right), \forall A \in \mathcal{B}(\mathbb{N} \times[0,1]),
$$


we have $\eta_{m} \leq \xi_{b}^{(n)}$ almost surely.

Proof. Given $n, m$ and $\boldsymbol{b} \in Q(n, m)$, define $\left(Y_{1}^{(n)}, Y_{2}^{(n)}, \ldots, Y_{n}^{(n)}\right)$ as in Definition 3.6.5 and, independently, define $2 m$ independent random variables $\left\{U_{i}\right\}_{i=1}^{m} \cup\left\{B_{i}\right\}_{i=1}^{m}$ such that each $U_{i}$ is uniformly distributed on $[0,1]$ and each $B_{i}$ is a Bernoulli random variable with $\mathbb{P}\left(B_{i}=1\right)=\frac{1}{q_{n}^{n}}$. We define the random vector $\left(V_{1}, V_{2}, \ldots, V_{n}\right)$ as follows,

- Sample the random vector $\left(Y_{1}^{(n)}, Y_{2}^{(n)}, \ldots, Y_{n}^{(n)}\right)$, say, we get $\left(Y_{1}^{(n)}, Y_{2}^{(n)}, \ldots, Y_{n}^{(n)}\right)=\left(y_{1}, y_{2}, \ldots, y_{n}\right)$.

- For $j \in[n] \backslash\left\{b_{i}\right\}_{i=1}^{m}$, let $V_{j}:=y_{j}$.

- For each $i \in[m]$, we resample $Y_{b_{i}}^{(n)}$ one by one, conditioned on the current value of other $Y_{j}^{(n)}$. Let $y_{b_{i}}^{\prime}$ denote the new value of $Y_{b_{i}}^{(n)}$ after the resampling and define $V_{b_{i}}:=y_{b_{i}}^{\prime}$. Specifically, for each $i \in[m]$, we sample a value $y_{b_{i}}^{\prime}$ according to the distribution of $Y_{b_{i}}^{(n)}$, conditioned on the event

$$
\left\{Y_{b_{j}}^{(n)}=y_{b_{j}}^{\prime} \text { for } \forall j<i \text { and } Y_{k}^{(n)}=y_{k} \text { for } \forall k \in[n] \backslash\left\{b_{j}\right\}_{j \in[i]}\right\} \text {. }
$$

- In each resampling step, say, resampling $Y_{b_{i}}^{(n)}$, let $\hat{\alpha}$ denote the above conditional distribution of $Y_{b_{i}}^{(n)}$. By Lemma 3.6.7, we know that that $\hat{\alpha}$ has density $f(y)$ with $f(y) \geq 1 / q_{n}^{n}$ almost surely. Hence, we can couple this resampling procedure with variables $U_{i}$ and $B_{i}$ in the same fashion as in the proof of Lemma 3.6.3, with $\alpha$ in that lemma being the uniform measure on $[0,1]$. Thus we have $\mathbb{1}_{A}\left(\left(i, V_{b_{i}}\right)\right) \geq$ $B_{i} \cdot \mathbb{1}_{A}\left(\left(i, U_{i}\right)\right)$ a.s. for any $A \in \mathcal{B}(\mathbb{N} \times[0,1])$.

It can be easily seen from the above procedure that $\left(V_{1}, V_{2}, \ldots, V_{n}\right)$ thus defined has the same distribution as $\left(Y_{1}^{(n)}, Y_{2}^{(n)}, \ldots, Y_{n}^{(n)}\right)$, and

$$
\eta_{m}(A)=\sum_{i=1}^{m} B_{i} \cdot \mathbb{1}_{A}\left(\left(i, U_{i}\right)\right) \leq \sum_{i=1}^{m} \mathbb{1}_{A}\left(\left(i, V_{b_{i}}\right)\right)=\xi_{b}^{(n)}(A) \text { a.s. }
$$

for any $A \in \mathcal{B}(\mathbb{N} \times[0,1])$. 
Lemma 3.6.9. Given $n \in \mathbb{N}$ and $0<q_{n} \leq 1$ such that $q_{n}^{n}>\frac{1}{2}$, for any $m \leq n$ and any $\boldsymbol{b}=\left(b_{1}, b_{2}, \ldots, b_{m}\right) \in Q(n, m)$, there exists a random vector $\left(V_{1}, V_{2}, \ldots, V_{n}\right) \in$ $[0,1]^{n}$ and $3 m$ independent random variables $\left\{U_{i}\right\}_{i=1}^{m} \cup\left\{U_{i}^{\prime}\right\}_{i=1}^{m} \cup\left\{B_{i}\right\}_{i=1}^{m}$ such that $\left(V_{1}, V_{2}, \ldots, V_{n}\right)$ has the same distribution as $\left(Y_{1}^{(n)}, Y_{2}^{(n)}, \ldots, Y_{n}^{(n)}\right)$, each $U_{i}, U_{i}^{\prime}$ are uniformly distributed on $[0,1]$ and each $B_{i}$ is a Bernoulli random variable with $\mathbb{P}\left(B_{i}=\right.$ $1)=\frac{1}{q_{n}^{n}}-1$. Moreover, if we define two point processes as follows,

$$
\begin{array}{rlrl}
\xi_{\boldsymbol{b}}^{(n)}(A):=\sum_{i=1}^{m} \mathbb{1}_{A}\left(\left(i, V_{b_{i}}\right)\right), & & \forall A \in \mathcal{B}(\mathbb{N} \times[0,1]) \\
\zeta_{m}(A):=\sum_{i=1}^{m} \mathbb{1}_{A}\left(\left(i, U_{i}^{\prime}\right)\right)+B_{i} \cdot \mathbb{1}_{A}\left(\left(i, U_{i}\right)\right), & \forall A \in \mathcal{B}(\mathbb{N} \times[0,1])
\end{array}
$$

we have $\xi_{b}^{(n)} \leq \zeta_{m}$ almost surely.

Proof. The proof of this lemma is similar to the proof of Lemma 3.6.8. Given $n, m$ and $\boldsymbol{b} \in Q(n, m)$, define $\left(Y_{1}^{(n)}, Y_{2}^{(n)}, \ldots, Y_{n}^{(n)}\right)$ as in Definition 3.6.5 and, independently, define $3 m$ independent random variables $\left\{U_{i}\right\}_{i=1}^{m} \cup\left\{U_{i}^{\prime}\right\}_{i=1}^{m} \cup\left\{B_{i}\right\}_{i=1}^{m}$ such that each $U_{i}, U_{i}^{\prime}$ are uniformly distributed on $[0,1]$ and each $B_{i}$ is a Bernoulli random variable with $\mathbb{P}\left(B_{i}=1\right)=1 / q_{n}^{n}-1$. Then we define the random vector $\left(V_{1}, V_{2}, \ldots, V_{n}\right)$ by the same steps as in the proof of Lemma 3.6.8, except that, in each resampling step, we couple the resampling of $Y_{b_{i}}^{(n)}$ with the variables $U_{i}, U_{i}^{\prime}$ and $B_{i}$ in the same fashion as in the proof of Lemma 3.6.4, with $\alpha$ in that lemma being the uniform measure on $[0,1]$. Note that the second inequality in Lemma 3.6.7 ensures that the conditions in Lemma 3.6.4 are met. Specifically, in each resampling step, let $f(y)$ denote the density of the conditional distribution of $Y_{b_{i}}^{(n)}$. Let $M, m$ be the maximum and minimum of $f(y)$ respectively. Define $\theta_{1}:=1-m$ and $\theta_{2}:=M-1$. Hence, $1-\theta_{1} \leq f(y) \leq 1+\theta_{2}$ almost surely and $\theta_{1}+\theta_{2}=M-m \leq 1 / q_{n}^{n}-1<1$.

Recall that $\mathcal{X}_{2}$ denotes the set of all Borel measures $\xi$ on $\mathbb{R}^{2}$ such that $\xi(A) \in$ $\{0,1,2, \ldots\}$ for any bounded Borel set $A$ in $\mathbb{R}^{2}$. 
Definition 3.6.10. For any $\xi \in \mathcal{X}_{2}$, we define the length of the longest increasing subsequence of $\xi$ as follows,

$$
\begin{aligned}
& \operatorname{LIS}(\xi):=\max \left\{k: \exists\left(x_{1}, y_{1}\right),\left(x_{2}, y_{2}\right), \ldots,\left(x_{k}, y_{k}\right) \in \mathbb{R}^{2}\right. \text { such that } \\
&\left.\xi\left(\left\{\left(x_{i}, y_{i}\right)\right\}\right) \geq 1, \forall i \in[k] \text { and }\left(x_{i}-x_{j}\right)\left(y_{i}-y_{j}\right)>0, \forall i \neq j\right\} .
\end{aligned}
$$

It is easily seen that the function $\operatorname{LIS}()$ is non-decreasing on $\mathcal{X}_{2}$ in the sense that, if $\xi, \zeta \in \mathcal{X}_{2}$ with $\xi \leq \zeta$, we have $\operatorname{LIS}(\xi) \leq \operatorname{LIS}(\zeta)$. Moreover, for any $n$ points $\left\{\left(x_{i}, y_{i}\right)\right\}_{i=1}^{n}$ in $\mathbb{R}^{2}$ such that $x_{i} \neq x_{j}$ and $y_{i} \neq y_{j}$ for all $i \neq j$, define the integer-valued measure $\xi$ as follows,

$$
\xi(A):=\sum_{i=1}^{n} \mathbb{1}_{A}\left(\left(x_{i}, y_{i}\right)\right), \quad \forall A \in \mathcal{B}\left(\mathbb{R}^{2}\right) .
$$

Then we have $\operatorname{LIS}(\xi)=\operatorname{LIS}\left(\left\{\left(x_{i}, y_{i}\right)\right\}_{i=1}^{n}\right)$, where the latter one is defined in Definition 3.1.1.

Lemma 3.6.11. Let $\left(V_{1}, \ldots, V_{n}\right)$ be a random vector which has the same distribution as $\left(Y_{1}^{(n)}, \ldots, Y_{n}^{(n)}\right)$. For any $m \leq n$ and $\boldsymbol{b}=\left(b_{1}, b_{2}, \ldots, b_{m}\right) \in Q(n, m)$, define the point process $\xi_{\boldsymbol{b}}^{(n)}$ as in the previous two lemmas, that is,

$$
\xi_{\boldsymbol{b}}^{(n)}(A):=\sum_{i=1}^{m} \mathbb{1}_{A}\left(\left(i, V_{b_{i}}\right)\right), \quad \forall A \in \mathcal{B}(\mathbb{N} \times[0,1]) .
$$

Then $\operatorname{LIS}\left(\xi_{\boldsymbol{b}}^{(n)}\right)$ and $\operatorname{LIS}\left(\pi_{\boldsymbol{b}}\right)$ have the same distribution, where $\pi \sim \mu_{n, q_{n}}$.

Proof. By the remarks after Definition 3.6.6, we have

$$
\Phi\left(\left\{\left(i, V_{b_{i}}\right)\right\}_{i=1}^{m}\right)=\Phi\left(\left(V_{b_{1}}, V_{b_{2}}, \ldots, V_{b_{m}}\right)\right)=\Phi\left(\left(V_{1}, V_{2}, \ldots, V_{n}\right)\right)_{b}
$$

where $\Phi\left(\left(V_{1}, V_{2}, \ldots, V_{n}\right)\right)$ in the last term has the distribution $\mu_{n, q_{n}}$. The lemma follows by the fact that

$$
\operatorname{LIS}\left(\xi_{\boldsymbol{b}}^{(n)}\right)=\operatorname{LIS}\left(\left\{\left(i, V_{b_{i}}\right)\right\}_{i=1}^{m}\right)=\operatorname{LIS}\left(\Phi\left(\left\{\left(i, V_{b_{i}}\right)\right\}_{i=1}^{m}\right)\right)
$$


Now we are in the position to prove Lemma 3.3.1 and Lemma 3.3.2. In the following, we use $\lambda_{n}$ to denote the uniform measure on $S_{n}$.

Proof of Lemma 3.3.1. The lemma can be divided into two parts. For the first part, we show that, for any sequence $\left\{k_{n}\right\}_{n=1}^{\infty}$ such that $k_{n} \in[n]$ and $\lim _{n \rightarrow \infty} k_{n}=\infty$,

$$
\lim _{n \rightarrow \infty} \max _{\boldsymbol{b} \in Q\left(n, k_{n}\right)} \mu_{n, q_{n}}\left(\pi \in S_{n}: \frac{\operatorname{LIS}\left(\pi_{\boldsymbol{b}}\right)}{\sqrt{k_{n}}}>2+\epsilon\right)=0
$$

for any $\epsilon>0$.

Since we have $q_{n} \geq 1$, by Corollary 3.2 .8 (a), for any $\boldsymbol{b} \in Q\left(n, k_{n}\right)$, there exist two random variables $(U, V)$ such that $U \sim \lambda_{k_{n}}, V$ has the same distribution as $\pi_{\boldsymbol{b}}$ with $\pi \sim \mu_{n, q_{n}}$ and $U \leq_{L} V$. Hence we have $\operatorname{LIS}(U) \geq \operatorname{LIS}(V)$, since LIS() is non-increasing on the poset $\left(S_{n}, \leq_{L}\right)$. Therefore, we have

$$
\mu_{n, q_{n}}\left(\pi \in S_{n}: \frac{\operatorname{LIS}\left(\pi_{\boldsymbol{b}}\right)}{\sqrt{k_{n}}}>2+\epsilon\right) \leq \lambda_{k_{n}}\left(\pi \in S_{k_{n}}: \frac{\operatorname{LIS}(\pi)}{\sqrt{k_{n}}}>2+\epsilon\right) .
$$

Then (3.111) follows by the result of Vershik and Kerov [17] that, under uniform measure, $\operatorname{LIS}(\pi) / \sqrt{n}$ converges in probability to 2 as $n$ goes to infinity. Note that (3.111) only depends on the fact that $q_{n} \geq 1$.

For the second part, we need to show that, for any $\epsilon>0$,

$$
\lim _{n \rightarrow \infty} \max _{\boldsymbol{b} \in Q\left(n, k_{n}\right)} \mu_{n, q_{n}}\left(\pi \in S_{n}: \frac{\operatorname{LIS}\left(\pi_{\boldsymbol{b}}\right)}{\sqrt{k_{n}}} \leq 2 e^{\frac{\beta}{2}}-\epsilon\right)=0 .
$$

Given $n>0$, for any $\boldsymbol{b} \in Q\left(n, k_{n}\right)$, by Lemma 3.6.11, $\operatorname{LIS}\left(\xi_{\boldsymbol{b}}^{(n)}\right)$ and $\operatorname{LIS}\left(\pi_{\boldsymbol{b}}\right)$ have the same distribution, where $\xi_{\boldsymbol{b}}^{(n)}$ is the point process as defined in that lemma. Moreover, by Lemma 3.6.8, there exists a point process $\eta_{k_{n}}$ such that $\eta_{k_{n}} \leq \xi_{\boldsymbol{b}}^{(n)}$ almost surely and $\eta_{k_{n}}$ is defined by

$$
\eta_{k_{n}}(A):=\sum_{i=1}^{k_{n}} B_{n, i} \cdot \mathbb{1}_{A}\left(\left(i, U_{i}\right)\right) \quad \forall A \in \mathcal{B}(\mathbb{N} \times[0,1])
$$

where $\left\{U_{i}\right\}_{i=1}^{k_{n}} \cup\left\{B_{n, i}\right\}_{i=1}^{k_{n}}$ are $2 k_{n}$ independent random variables with each $U_{i}$ being uniformly distributed on $[0,1]$ and each $B_{n, i}$ being a Bernoulli random variable with 
$\mathbb{P}\left(B_{n, i}=1\right)=1 / q_{n}^{n}$.

Hence, we have

$$
\begin{aligned}
\mu_{n, q_{n}}\left(\pi \in S_{n}: \frac{\operatorname{LIS}\left(\pi_{b}\right)}{\sqrt{k_{n}}} \leq 2 e^{\frac{\beta}{2}}-\epsilon\right) & =\mathbb{P}\left(\frac{\operatorname{LIS}\left(\xi_{b}^{(n)}\right)}{\sqrt{k_{n}}} \leq 2 e^{\frac{\beta}{2}}-\epsilon\right) \\
& \leq \mathbb{P}\left(\frac{\operatorname{LIS}\left(\eta_{k_{n}}\right)}{\sqrt{k_{n}}} \leq 2 e^{\frac{\beta}{2}}-\epsilon\right) .
\end{aligned}
$$

Here the last inequality follows by the monotonicity of $\operatorname{LIS}()$ on $\mathcal{X}_{2}$.

We complete the proof of (3.112) as well as Lemma 3.3.1 by showing the following,

$$
\lim _{n \rightarrow \infty} \mathbb{P}\left(\frac{\operatorname{LIS}\left(\eta_{k_{n}}\right)}{\sqrt{k_{n}}}>2 e^{\frac{\beta}{2}}-\epsilon\right)=1,
$$

for any $\epsilon>0$.

From the inequality $\ln (1+x) \leq x$ for all $x>-1$, we have

$$
\frac{1}{q_{n}^{n}}=e^{-n \ln q_{n}} \geq e^{-n\left(q_{n}-1\right)}=e^{n\left(1-q_{n}\right)} .
$$

Since $\liminf \operatorname{in}_{n \rightarrow \infty} n\left(1-q_{n}\right)=\beta$, for any $\epsilon_{1}>0$, there exists $N_{1}>0$ such that, for any $n>N_{1}$, we have $1 / q_{n}^{n}>e^{\beta-\epsilon_{1}}$. Thus, by the law of large numbers, we have

$$
\lim _{n \rightarrow \infty} \mathbb{P}\left(\sum_{i=1}^{k_{n}} B_{n, i}>k_{n} e^{\beta-\epsilon_{1}}\right)=1 .
$$

Here we use the fact that $\lim _{n \rightarrow \infty} k_{n}=\infty$. Given $\boldsymbol{U}=\left(U_{1}, \ldots, U_{k_{n}}\right)$ and $\boldsymbol{B}=$ $\left(B_{n, 1}, \ldots, B_{n, k_{n}}\right)$, let $\Lambda(\boldsymbol{U}, \boldsymbol{B})$ denote the set of points in $\mathbb{R}^{2}$ defined by

$$
\Lambda(\boldsymbol{U}, \boldsymbol{B}):=\left\{\left(i, U_{i}\right): i \in\left[k_{n}\right] \text { and } B_{n, i}=1\right\} .
$$

By the definition of $\eta_{k_{n}}$ and Definition 3.6.10, we have

$$
\operatorname{LIS}\left(\eta_{k_{n}}\right)=\operatorname{LIS}(\Lambda(\boldsymbol{U}, \boldsymbol{B})) .
$$

Moreover, conditioned on $\sum_{i=1}^{k_{n}} B_{n, i}=m$, by the independence of $\boldsymbol{U}$ and $\boldsymbol{B}$, it is easily seen that $\operatorname{LIS}(\Lambda(\boldsymbol{U}, \boldsymbol{B}))$ has the same distribution as $\operatorname{LIS}(\pi)$ with $\pi \sim \lambda_{m}$.

For any $0<\epsilon_{2}, \epsilon_{3}<1$, by the result of Vershik and Kerov [17] again, there exists $M>0$ such that, for any $m>M$,

$$
\lambda_{m}\left(\frac{\operatorname{LIS}(\pi)}{\sqrt{m}}>2-\epsilon_{2}\right)>1-\epsilon_{3} .
$$


Since $\lim _{n \rightarrow \infty} k_{n}=\infty$ and (3.115), there exists $N>N_{1}$ such that, for any $n>N$, we have

$$
k_{n} e^{\beta-\epsilon_{1}}>M \quad \text { and } \quad \mathbb{P}\left(\sum_{i=1}^{k_{n}} B_{n, i}>k_{n} e^{\beta-\epsilon_{1}}\right)>1-\epsilon_{3} .
$$

Let $s=\left\lfloor k_{n} e^{\beta-\epsilon_{1}}\right\rfloor+1$. For any $n>N$, we have

$$
\begin{aligned}
& \mathbb{P}\left(\operatorname{LIS}\left(\eta_{k_{n}}\right)>\left(2-\epsilon_{2}\right) \sqrt{k_{n} e^{\beta-\epsilon_{1}}}\right) \\
\geq & \sum_{m=s}^{k_{n}} \mathbb{P}\left(\operatorname{LIS}\left(\eta_{k_{n}}\right)>\left(2-\epsilon_{2}\right) \sqrt{k_{n} e^{\beta-\epsilon_{1}}} \mid \sum_{i=1}^{k_{n}} B_{n, i}=m\right) \mathbb{P}\left(\sum_{i=1}^{k_{n}} B_{n, i}=m\right) \\
\geq & \sum_{m=s}^{k_{n}} \mathbb{P}\left(\operatorname{LIS}\left(\eta_{k_{n}}\right)>\left(2-\epsilon_{2}\right) \sqrt{m} \mid \sum_{i=1}^{k_{n}} B_{n, i}=m\right) \mathbb{P}\left(\sum_{i=1}^{k_{n}} B_{n, i}=m\right) \\
= & \sum_{m=s}^{k_{n}} \lambda_{m}\left(\operatorname{LIS}(\pi)>\left(2-\epsilon_{2}\right) \sqrt{m}\right) \mathbb{P}\left(\sum_{i=1}^{k_{n}} B_{n, i}=m\right) \\
> & \left(1-\epsilon_{3}\right) \sum_{m=s}^{k_{n}} \mathbb{P}\left(\sum_{i=1}^{k_{n}} B_{n, i}=m\right) \\
= & \left(1-\epsilon_{3}\right) \mathbb{P}\left(\sum_{i=1}^{k_{n}} B_{n, i}>k_{n} e^{\beta-\epsilon_{1}}\right) \\
> & \left(1-\epsilon_{3}\right)^{2} .
\end{aligned}
$$

Here the second inequality follows since $m \geq s \geq k_{n} e^{\beta-\epsilon_{1}}$. The third inequailty follows from (3.116) and the fact that $m \geq k_{n} e^{\beta-\epsilon_{1}}>M$. Therefore, we have shown that $\lim _{n \rightarrow \infty} \mathbb{P}\left(\operatorname{LIS}\left(\eta_{k_{n}}\right)>\left(2-\epsilon_{2}\right) \sqrt{k_{n} e^{\beta-\epsilon_{1}}}\right)=1$, and (3.114) follows from the fact that, by choosing $\epsilon_{1}$ and $\epsilon_{2}$ small enough, $\left(2-\epsilon_{2}\right) \sqrt{e^{\beta-\epsilon_{1}}}$ can be arbitrarily close to $2 e^{\frac{\beta}{2}}$.

The proof of Lemma 3.3.2 is similar to the proof of Lemma 3.3.1.

Proof of Lemma 3.3.2. Again, we split the proof into two parts. For the first part, we need to show that, for any $\epsilon>0$,

$$
\lim _{n \rightarrow \infty} \max _{\boldsymbol{b} \in Q\left(n, k_{n}\right)} \mu_{n, q_{n}}\left(\pi \in S_{n}: \frac{\operatorname{LIS}\left(\pi_{\boldsymbol{b}}\right)}{\sqrt{k_{n}}}<2-\epsilon\right)=0 .
$$

For this part, since $0<q_{n} \leq 1$, we use Corollary 3.2.8 (b). For any $\boldsymbol{b} \in Q\left(n, k_{n}\right)$, there exist two random variables $(U, V)$ such that $U \sim \lambda_{k_{n}}, V$ has the same distribution 
as $\pi_{\boldsymbol{b}}$ with $\pi \sim \mu_{n, q_{n}}$ and $V \leq_{L} U$. Hence we have $\operatorname{LIS}(V) \geq \operatorname{LIS}(U)$, since $\operatorname{LIS}()$ is non-increasing on the poset $\left(S_{n}, \leq_{L}\right)$. Therefore, we have

$$
\mu_{n, q_{n}}\left(\pi \in S_{n}: \frac{\operatorname{LIS}\left(\pi_{\boldsymbol{b}}\right)}{\sqrt{k_{n}}}<2-\epsilon\right) \leq \lambda_{k_{n}}\left(\pi \in S_{k_{n}}: \frac{\operatorname{LIS}(\pi)}{\sqrt{k_{n}}}<2-\epsilon\right) .
$$

Then (3.117) follows by the result of Vershik and Kerov [17] that, under uniform measure, $\operatorname{LIS}(\pi) / \sqrt{n}$ converges in probability to 2 as $n$ goes to infinity. Note that (3.117) only depends on the fact that $0<q_{n} \leq 1$.

For the second part, we need to show that, for any $\epsilon>0$,

$$
\lim _{n \rightarrow \infty} \max _{\boldsymbol{b} \in Q\left(n, k_{n}\right)} \mu_{n, q_{n}}\left(\pi \in S_{n}: \frac{\operatorname{LIS}\left(\pi_{\boldsymbol{b}}\right)}{\sqrt{k_{n}}} \geq 2 e^{\frac{\beta}{2}}+\epsilon\right)=0 .
$$

First, we point out that, for any sequence $\left\{q_{n}\right\}_{n=1}^{\infty}$ with $0<q_{n} \leq 1$ and $\lim \sup _{n \rightarrow \infty} n(1-$ $\left.q_{n}\right)=\beta<\ln 2$, we have

$$
\limsup _{n \rightarrow \infty} \frac{1}{q_{n}^{n}}=e^{\limsup _{n \rightarrow \infty}-n \ln q_{n}}=e^{\limsup _{n \rightarrow \infty} n\left(1-q_{n}\right)}=e^{\beta}<2 .
$$

Here the second equality follows from the fact that $\lim _{x \rightarrow 1} \frac{\ln x}{x-1}=1$. Thus, for any $0<\epsilon_{1}<\ln 2-\beta$, there exists $N_{1}>0$ such that, for all $n>N_{1}$, we have $1 / q_{n}^{n}<e^{\beta+\epsilon_{1}}$.

Given $n>N_{1}$, for any $\boldsymbol{b} \in Q\left(n, k_{n}\right)$, by Lemma 3.6.11, $\operatorname{LIS}\left(\xi_{\boldsymbol{b}}^{(n)}\right)$ and $\operatorname{LIS}\left(\pi_{\boldsymbol{b}}\right)$ have the same distribution, where $\xi_{b}^{(n)}$ is the point process as defined in that lemma. Moreover, by Lemma 3.6.9, there exists a point process $\zeta_{k_{n}}$ such that $\xi_{\boldsymbol{b}}^{(n)} \leq \zeta_{k_{n}}$ almost surely and $\zeta_{k_{n}}$ is defined by

$$
\zeta_{k_{n}}(A):=\sum_{i=1}^{k_{n}} \mathbb{1}_{A}\left(\left(i, U_{i}^{\prime}\right)\right)+B_{n, i} \cdot \mathbb{1}_{A}\left(\left(i, U_{i}\right)\right) \quad \forall A \in \mathcal{B}(\mathbb{N} \times[0,1]),
$$

where $\left\{U_{i}\right\}_{i=1}^{k_{n}} \cup\left\{U_{i}^{\prime}\right\}_{i=1}^{k_{n}} \cup\left\{B_{n, i}\right\}_{i=1}^{k_{n}}$ are $3 k_{n}$ independent random variables with each $U_{i}, U_{i}^{\prime}$ being uniformly distributed on $[0,1]$ and each $B_{n, i}$ being a Bernoulli random variable with $\mathbb{P}\left(B_{n, i}=1\right)=\frac{1}{q_{n}^{n}}-1$.

Hence, we have

$$
\mu_{n, q_{n}}\left(\pi \in S_{n}: \frac{\operatorname{LIS}\left(\pi_{\boldsymbol{b}}\right)}{\sqrt{k_{n}}} \geq 2 e^{\frac{\beta}{2}}+\epsilon\right)=\mathbb{P}\left(\frac{\operatorname{LIS}\left(\xi_{b}^{(n)}\right)}{\sqrt{k_{n}}} \geq 2 e^{\frac{\beta}{2}}+\epsilon\right)
$$




$$
\leq \mathbb{P}\left(\frac{\operatorname{LIS}\left(\zeta_{k_{n}}\right)}{\sqrt{k_{n}}} \geq 2 e^{\frac{\beta}{2}}+\epsilon\right)
$$

Here the last inequality follows by the monotonicity of $\operatorname{LIS}()$ on $\mathcal{X}_{2}$.

We complete the proof of (3.118) as well as Lemma 3.3.2 by showing that, for any $\epsilon>0$

$$
\lim _{n \rightarrow \infty} \mathbb{P}\left(\frac{\operatorname{LIS}\left(\zeta_{k_{n}}\right)}{\sqrt{k_{n}}}<2 e^{\frac{\beta}{2}}+\epsilon\right)=1
$$

First, since, for all $n>N_{1}$, we have $\mathbb{P}\left(B_{n, i}=1\right)=1 / q_{n}^{n}-1<e^{\beta+\epsilon_{1}}-1$, by the law of large numbers, we get

$$
\lim _{n \rightarrow \infty} \mathbb{P}\left(\sum_{i=1}^{k_{n}} B_{n, i}<k_{n}\left(e^{\beta+\epsilon_{1}}-1\right)\right)=1
$$

Here we use the fact that $\lim _{n \rightarrow \infty} k_{n}=\infty$. Given $\boldsymbol{U}^{\prime}=\left(U_{1}^{\prime}, \ldots, U_{k_{n}}^{\prime}\right), \boldsymbol{U}=\left(U_{1}, \ldots, U_{k_{n}}\right)$ and $\boldsymbol{B}=\left(B_{n, 1}, \ldots, B_{n, k_{n}}\right)$, let $\Lambda\left(\boldsymbol{U}^{\prime}, \boldsymbol{U}, \boldsymbol{B}\right)$ denote the set of points in $\mathbb{R}^{2}$ defined by

$$
\Lambda\left(\boldsymbol{U}^{\prime}, \boldsymbol{U}, \boldsymbol{B}\right):=\left\{\left(i, U_{i}\right): i \in\left[k_{n}\right] \text { and } B_{n, i}=1\right\} \bigcup\left\{\left(i, U_{i}^{\prime}\right): i \in\left[k_{n}\right]\right\}
$$

By the definition of $\zeta_{k_{n}}$ and Definition 3.6.10, we have

$$
\operatorname{LIS}\left(\zeta_{k_{n}}\right)=\operatorname{LIS}\left(\Lambda\left(\boldsymbol{U}^{\prime}, \boldsymbol{U}, \boldsymbol{B}\right)\right)
$$

Based on $\boldsymbol{U}^{\prime}, \boldsymbol{U}$ and $\boldsymbol{B}$, define another set of points in $\mathbb{R}^{2}$ as follows,

$$
\Lambda^{+}\left(\boldsymbol{U}^{\prime}, \boldsymbol{U}, \boldsymbol{B}\right):=\left\{\left(i+1 / 2, U_{i}\right): i \in\left[k_{n}\right] \text { and } B_{n, i}=1\right\} \bigcup\left\{\left(i, U_{i}^{\prime}\right): i \in\left[k_{n}\right]\right\}
$$

Then, we have

$$
\operatorname{LIS}\left(\Lambda\left(\boldsymbol{U}^{\prime}, \boldsymbol{U}, \boldsymbol{B}\right)\right) \leq \operatorname{LIS}\left(\Lambda^{+}\left(\boldsymbol{U}^{\prime}, \boldsymbol{U}, \boldsymbol{B}\right)\right)
$$

Since, by Definition 3.1.1, no two points with the same $x$ coordinates can be both within an increasing subsequence, by increasing the $x$ coordinates of those points in $\Lambda\left(\boldsymbol{U}^{\prime}, \boldsymbol{U}, \boldsymbol{B}\right)$ which reside on the same vertical line as other points by $1 / 2$, the relative ordering of the shifted point with other points does not change, except the one which has the same $x$ coordinate when unshifted. Combining (3.122) and (3.123), we have

$$
\operatorname{LIS}\left(\zeta_{k_{n}}\right) \leq \operatorname{LIS}\left(\Lambda^{+}\left(\boldsymbol{U}^{\prime}, \boldsymbol{U}, \boldsymbol{B}\right)\right)
$$


Moreover, conditioned on $\sum_{i=1}^{k_{n}} B_{n, i}=m$, by independence of $\boldsymbol{U}^{\prime}, \boldsymbol{U}$ and $\boldsymbol{B}$, it is easily seen that $\operatorname{LIS}\left(\Lambda^{+}\left(\boldsymbol{U}^{\prime}, \boldsymbol{U}, \boldsymbol{B}\right)\right)$ has the same distribution as $\operatorname{LIS}(\pi)$ with $\pi \sim \lambda_{k_{n}+m}$. For any $0<\epsilon_{2}, \epsilon_{3}<1$, by the result of Vershik and Kerov [17] again, there exists $M>0$ such that, for any $k>M$,

$$
\lambda_{k}\left(\frac{\operatorname{LIS}(\pi)}{\sqrt{k}}<2+\epsilon_{2}\right)>1-\epsilon_{3} .
$$

Since $\lim _{n \rightarrow \infty} k_{n}=\infty$ and (3.121), there exists $N>N_{1}$ such that, for any $n>N$, we have

$$
k_{n}>M \quad \text { and } \quad \mathbb{P}\left(\sum_{i=1}^{k_{n}} B_{n, i}<k_{n}\left(e^{\beta+\epsilon_{1}}-1\right)\right)>1-\epsilon_{3} .
$$

Let $s=\left\lceil k_{n}\left(e^{\beta+\epsilon_{1}}-1\right)\right\rceil-1$. For any $n>N$, we have

$$
\begin{aligned}
& \mathbb{P}\left(\operatorname{LIS}\left(\zeta_{k_{n}}\right)<\left(2+\epsilon_{2}\right) \sqrt{k_{n} e^{\beta+\epsilon_{1}}}\right) \\
\geq & \sum_{m=0}^{s} \mathbb{P}\left(\operatorname{LIS}\left(\zeta_{k_{n}}\right)<\left(2+\epsilon_{2}\right) \sqrt{k_{n} e^{\beta+\epsilon_{1}}} \mid \sum_{i=1}^{k_{n}} B_{n, i}=m\right) \mathbb{P}\left(\sum_{i=1}^{k_{n}} B_{n, i}=m\right) \\
\geq & \sum_{m=0}^{s} \mathbb{P}\left(\operatorname{LIS}\left(\zeta_{k_{n}}\right)<\left(2+\epsilon_{2}\right) \sqrt{k_{n}+m} \mid \sum_{i=1}^{k_{n}} B_{n, i}=m\right) \mathbb{P}\left(\sum_{i=1}^{k_{n}} B_{n, i}=m\right) \\
\geq & \sum_{m=0}^{s} \mathbb{P}\left(\operatorname{LIS}\left(\Lambda^{+}\left(\boldsymbol{U}^{\prime}, \boldsymbol{U}, \boldsymbol{B}\right)\right)<\left(2+\epsilon_{2}\right) \sqrt{k_{n}+m} \mid \sum_{i=1}^{k_{n}} B_{n, i}=m\right) \\
= & \sum_{m=0}^{s} \lambda_{k_{n}+m}\left(\operatorname{LIS}(\pi)<\left(2+\epsilon_{2}\right) \sqrt{k_{n}+m}\right) \mathbb{P}\left(\sum_{i=1}^{k_{n}} B_{n, i}=m\right) \\
> & \left(1-\epsilon_{3}\right) \sum_{m=0}^{s} \mathbb{P}\left(\sum_{i=1}^{k_{n}} B_{n, i}=m\right) \\
= & \left(1-\epsilon_{3}\right) \mathbb{P}\left(\sum_{i=1}^{k_{n}} B_{n, i}<k_{n}\left(e^{\beta+\epsilon_{1}}-1\right)\right) \\
> & \left(1-\epsilon_{3}\right)^{2} .
\end{aligned}
$$

The second inequality follows because

$$
k_{n}+m \leq k_{n}+s<k_{n}+k_{n}\left(e^{\beta+\epsilon_{1}}-1\right)=k_{n} e^{\beta+\epsilon_{1}},
$$

and the third inequality follows from (3.124). Therefore, we have shown that

$$
\lim _{n \rightarrow \infty} \mathbb{P}\left(\operatorname{LIS}\left(\zeta_{k_{n}}\right)<\left(2+\epsilon_{2}\right) \sqrt{k_{n} e^{\beta+\epsilon_{1}}}\right)=1
$$


(3.120) follows from the fact that, by choosing $\epsilon_{1}$ and $\epsilon_{2}$ small enough, $\left(2+\epsilon_{2}\right) \sqrt{e^{\beta+\epsilon_{1}}}$ can be arbitrarily close to $2 e^{\frac{\beta}{2}}$. 


\section{Chapter 4}

\section{WEAK LAW OF LARGE NUMBERS WHEN NEITHER PERMUTATION IS CLOSE TO UNIFORMLY RANDOM}

In this chapter we prove Theorem 5, the weak law of large numbers for the LCS of two independent Mallows permutations when $q_{n}=q_{n}^{\prime}, q_{n} \rightarrow 1$ and $n\left(1-q_{n}\right) \rightarrow \infty$. Since $\operatorname{LCS}(\pi, \tau)$ has the same distribution as $\operatorname{LIS}(\pi, \tau)$, we once again reduce the LCS problem to the LIS problem. The remainder of the proof follows the approach used in [6] to prove a weak law for the LIS of a Mallows permutation (Theorem 1.1.9). We approximate $\operatorname{LIS}(\pi, \tau)$ by the sum of $\operatorname{LIS}\left(\pi_{B_{i}}, \tau_{B_{i}}\right)$, where $\left\{B_{i}\right\}$ are disjoint blocks of indices which partition $[n]$. We choose the size of $B_{i}$ such that the limiting size of $\operatorname{LIS}\left(\pi_{B_{i}}, \tau_{B_{i}}\right)$ can be obtained from Corollary 3. We believe that the approach can be extended to the case that $\lim _{n \rightarrow \infty} \frac{q_{n}}{q_{n}^{\prime}}=1$, but to keep the computation simple we restrict to the case that $q_{n}=q_{n}^{\prime}$.

\subsection{Mallows process}

In this section we describe a random process on permutations which was known to Mallows [20], and is termed as Mallows process in [6]. Given $q>0$, the $q$-Mallows process is a permutation-valued stochastic process $\left(p_{n}\right)_{n \geq 1}$, where $p_{n} \in S_{n}$. The process is initialized by setting $p_{1}$ to be the only permutation on one element. The process iteratively constructs $p_{n}$ from $p_{n-1}$ and an independent random variable $p_{n}(n)$ distributed as a truncated geometric. Precisely, let $\left\{p_{n}(n)\right\}_{n \geq 1}$ be a sequence of independent random variables with the distributions

$$
\mathbb{P}\left(p_{n}(n)=j\right)=\frac{q^{j-1}}{1+q+\cdots+q^{n-1}}=\frac{(1-q) q^{j-1}}{1-q^{n}}, \quad \forall 1 \leq j \leq n .
$$


Each permutation $p_{n}$ is defined iteratively by

$$
p_{n}(i)= \begin{cases}p_{n-1}(i), & \text { when } p_{n-1}(i)<p_{n}(n) \\ p_{n-1}(i)+1, & \text { when } p_{n-1}(i) \geq p_{n}(n) \\ p_{n}(n), & \text { when } i=n .\end{cases}
$$

The Mallows process constructed as above has the following property (cf. Lemma 2.1 in $[6])$.

Lemma 4.1.1. Let $q>0$ and let $\left\{p_{n}\right\}_{n \geq 1}$ be the $q$-Mallows process. Then $p_{n}$ is distributed according to $\mu_{n, 1 / q}$.

The next lemma says that $p_{i}(i)$ is determined by the value of $p_{n}$ on $[i]$.

Lemma 4.1.2. For any $1 \leq i \leq n$, we have

$$
i-p_{i}(i)=\sum_{t=1}^{i} \mathbb{1}\left(p_{n}(t)>p_{n}(i)\right) .
$$

Proof. By the definition of Mallows process, $p_{i}$ is a permutation in $S_{i}$. Hence we have

$$
\begin{aligned}
p_{i}(i) & =\sum_{t=1}^{i} \mathbb{1}\left(p_{i}(t) \leq p_{i}(i)\right) \\
& =\sum_{t=1}^{i} \mathbb{1}\left(p_{n}(t) \leq p_{n}(i)\right) .
\end{aligned}
$$

Here the last equality follows since the relative ordering of previous indices will not change by the following updates. Thus

$$
i-p_{i}(i)=\sum_{t=1}^{i} 1-\mathbb{1}\left(p_{n}(t) \leq p_{n}(i)\right)=\sum_{t=1}^{i} \mathbb{1}\left(p_{n}(t)>p_{n}(i)\right) .
$$

A direct corollary of Lemma 4.1.2 is that the number of inversion of $p_{n}$ can be written as a function of $p_{i}(i)$.

\section{Corollary 4.1.3.}

$$
l\left(p_{n}\right)=\frac{(n+1) n}{2}-\sum_{i=1}^{n} p_{i}(i)
$$


Lemma 4.1.4. For any $1 \leq i \leq n$, we have

$$
p_{n}(i)=p_{i}(i)+n-i-\sum_{t=i+1}^{n} \mathbb{1}\left(p_{n}(t)>p_{n}(i)\right) .
$$

Moreover, if $k \in[n] \backslash\left\{p_{n}(t): i+1 \leq t \leq n\right\}$ satisfy the following equation,

$$
k=p_{i}(i)+n-i-\sum_{t=i+1}^{n} \mathbb{1}\left(p_{n}(t)>k\right) .
$$

we have $k=p_{n}(i)$.

Proof. Since $p_{n}$ is a permutation in $S_{n}$, we have

$$
p_{n}(i)=n-\sum_{t=1}^{n} \mathbb{1}\left(p_{n}(t)>p_{n}(i)\right) \text {. }
$$

Hence (4.3) follows from (4.1). We prove the second claim by contradiction. Suppose we have $j<k$ with $j, k \in[n] \backslash\left\{p_{n}(t): i+1 \leq t \leq n\right\}$ such that

$$
\begin{aligned}
& j=p_{i}(i)+n-i-\sum_{t=i+1}^{n} \mathbb{1}\left(p_{n}(t)>j\right), \\
& k=p_{i}(i)+n-i-\sum_{t=i+1}^{n} \mathbb{1}\left(p_{n}(t)>k\right) .
\end{aligned}
$$

By subtracting these two equations, we have

$$
k-j=\sum_{t=i+1}^{n} \mathbb{1}\left(j<p_{n}(t) \leq k\right)=\sum_{t=i+1}^{n} \mathbb{1}\left(j<p_{n}(t) \leq k-1\right),
$$

which is a contradiction. Because $\left\{p_{n}(t): i+1 \leq t \leq n\right\}$ are distinct numbers and there are only $k-j-1$ slots within $(j, k-1]$.

Let $\pi_{n}$ and $\tau_{n}$ be as defined in Theorem 5. By Lemma 3.2.11, for any $n \geq 1$, there exists a coupling $\left(\pi_{n}, \tau_{n}, Z_{n}\right)$ such that $Z_{n} \sim \mu_{n, q}$ and

$$
\operatorname{LIS}\left(\pi_{n}, \tau_{n}\right) \leq \operatorname{LIS}\left(Z_{n}\right)
$$

In [6] Section 5.1, the authors show that, given $p>0$, when $q$ is sufficiently close to 1 , the family of random variables $\left\{\left|\frac{\operatorname{LIS}\left(Z_{n}\right)}{n \sqrt{1-q}}\right|^{p}\right\}$ indexed by $q$ is uniformly integrable. Hence 
by (4.4), the family of random variables $\left\{\left|\frac{\operatorname{LIS}\left(\pi_{n}, \tau_{n}\right)}{n \sqrt{1-q}}\right|^{p}\right\}$ is also uniformly integrable. In the following we show that

$$
\frac{\operatorname{LIS}\left(\pi_{n}, \tau_{n}\right)}{n \sqrt{1-q_{n}}} \stackrel{L_{1}}{\longrightarrow} \frac{\sqrt{6}}{3},
$$

as $n \rightarrow \infty$. Then, by the uniform integrability of $\left\{\left|\frac{\operatorname{LIS}\left(\pi_{n}, \tau_{n}\right)}{n \sqrt{1-q}}\right|^{p}\right\}$, for any $p>0$, we have

$$
\frac{\operatorname{LIS}\left(\pi_{n}, \tau_{n}\right)}{n \sqrt{1-q_{n}}} \stackrel{L_{p}}{\longrightarrow} \frac{\sqrt{6}}{3},
$$

as $n \rightarrow \infty$. Theorem 5 follows from Corollary 3.1.4 and the fact that $\left(\pi_{n}, \tau_{n}\right)$ has the same distribution as $\left(\pi_{n}^{-1}, \tau_{n}^{-1}\right)$. The proof of (4.5) follows the approach developed in [6] in which the authors prove a similar result for the length of the LIS of a Mallows permutation.

\subsection{Block decomposition}

Let $n=n(q)$ be a function of $q$ such that

$$
\lim _{q \rightarrow 1} n=\infty, \quad \text { and } \quad \lim _{q \rightarrow 1} n(1-q)=\infty
$$

Let $\pi \sim \mu_{n, q}, \tau \sim \mu_{n, q}$ and $\pi$ and $\tau$ are independent. To prove (4.5), it suffices to show that

$$
\frac{\operatorname{LIS}(\pi, \tau)}{n \sqrt{1-q}} \stackrel{L_{1}}{\longrightarrow} \frac{\sqrt{6}}{3},
$$

as $q \rightarrow 1$. In the following, we will partition $[n]$ into blocks of size $\frac{\beta}{1-q}$ for some large $\beta$. We consider the LIS formed by $\pi$ and $\tau$ when restricted to each blocks and show that the concatenation of these increasing subsequences within each block is close to $\operatorname{LIS}(\pi, \tau)$.

Given $\beta>0$, define a function $\beta(q)$ such that $\frac{\beta(q)}{1-q}$ is an integer and $\beta(q) \rightarrow \beta$ as $q \rightarrow 1$. Define

$$
m:=\left\lfloor\frac{n(1-q)}{\beta(q)}\right\rfloor .
$$

For $1 \leq i \leq m$ define

$$
B_{i}:=\left((i-1) \frac{\beta(q)}{1-q}+1, \ldots, i \frac{\beta(q)}{1-q}\right)
$$


Hence, each $B_{i}$ is a block of consecutive integers of size $\frac{\beta(q)}{1-q}$. To make $\left\{B_{i}\right\}$ a partition of $[n]$, define $B_{m+1}:=\left(m \frac{\beta(q)}{1-q}+1, \ldots, n\right)$. For $1 \leq i \leq m+1$, let

$$
X_{i}:=\operatorname{LIS}\left(\pi_{B_{i}}, \tau_{B_{i}}\right)
$$

be the length of the longest increasing subsequence of the restriction of $\pi$ and $\tau$ to $B_{i}$. By Lemma 1.1.5, the $X_{i}$ are independent. By Lemma 1.1.6, each $X_{i}$ has the distribution of the LIS of two independent Mallows permutations of size $\frac{\beta(q)}{1-q}$ and parameter $q$. Moreover, by Lemma 1.1.4, and using Corollary 3.1.4 in another direction, $X_{i}$ has the distribution of the LCS of two independent Mallows permutations of size $\frac{\beta(q)}{1-q}$ and parameter $q$.

By triangle inequality, we have

$$
\left|\frac{\operatorname{LIS}(\pi, \tau)}{n \sqrt{1-q}}-\frac{\sqrt{6}}{3}\right| \leq\left|\frac{\operatorname{LIS}(\pi, \tau)-\sum_{i=1}^{m} X_{i}}{n \sqrt{1-q}}\right|+\left|\frac{\sum_{i=1}^{m} X_{i}}{n \sqrt{1-q}}-\frac{\sqrt{6}}{3}\right| .
$$

We will prove that

$$
\begin{gathered}
\varlimsup_{\beta \rightarrow \infty} \varlimsup_{q \rightarrow 1} \mathbb{E}\left(\left|\frac{\operatorname{LIS}(\pi, \tau)-\sum_{i=1}^{m} X_{i}}{n \sqrt{1-q}}\right|\right)=0, \\
\varlimsup_{\beta \rightarrow \infty} \varlimsup_{q \rightarrow 1} \mathbb{E}\left(\left|\frac{\sum_{i=1}^{m} X_{i}}{n \sqrt{1-q}}-\frac{\sqrt{6}}{3}\right|\right)=0 .
\end{gathered}
$$

These equalities imply that

$$
\varlimsup_{\beta \rightarrow \infty} \varlimsup_{q \rightarrow 1} \mathbb{E}\left(\left|\frac{\operatorname{LIS}(\pi, \tau)}{n \sqrt{1-q}}-\frac{\sqrt{6}}{3}\right|\right)=0,
$$

and since $\pi$ and $\tau$ do not depend on $\beta$, we have

$$
\lim _{q \rightarrow 1} \mathbb{E}\left(\left|\frac{\operatorname{LIS}(\pi, \tau)}{n \sqrt{1-q}}-\frac{\sqrt{6}}{3}\right|\right)=0
$$

which is exactly (4.8).

Since $\left\{B_{i}\right\}$ partition $[n]$, it follows trivially that

$$
\operatorname{LIS}(\pi, \tau) \leq \sum_{i=1}^{m+1} X_{i}
$$


We will show a bound in the other direction by using the $q$-Mallows process. Given two independent $q$-Mallows processes $\left\{p_{i}\right\}$ and $\left\{p_{i}^{\prime}\right\}$, define two permutations $\pi$ and $\tau$ by

$$
\pi(j):=n+1-p_{n}(j), \quad \tau(j):=n+1-p_{n}^{\prime}(j)
$$

for $1 \leq j \leq n$. By Lemma 4.1.1 and Lemma 1.1.4, it follows that $\pi \sim \mu_{n, q}$ and $\tau \sim \mu_{n, q}$. Let $a=a(\beta)>0$ be any function of $\beta$ satisfying

$$
a \rightarrow \infty \quad \text { and } \quad \frac{a}{\beta} \rightarrow 0, \quad \text { as } \beta \rightarrow \infty
$$

For each $i \in[m]$ define

$$
E_{i}:=\left\{j \in B_{i}: p_{\max B_{i}}(j) \leq \frac{a}{1-q}\right\}, \quad F_{i}:=\left\{j \in B_{i}: p_{j}(j)>\frac{a}{1-q}\right\} .
$$

That is $E_{i}$ consists of those indices in $B_{i}$ at which the first $q$-Mallows process is at most $\frac{a}{1-q}$ after the entire block $B_{i}$ is assigned. $F_{i}$ consists of those indices in $B_{i}$ at which its initial position is greater than $\frac{a}{1-q}$. For the second $q$-Mallows process, we define $E_{i}^{\prime}$ and $F_{i}^{\prime}$ similarly.

Let $I_{i}=\left(i_{1}, \ldots, i_{k}\right) \subset B_{i}$ be the indices of an arbitrary longest increasing subsequence of $\pi$ and $\tau$ in the restriction of $B_{i}$. That is $\pi\left(i_{j}\right)<\pi\left(i_{j+1}\right)$ and $\tau\left(i_{j}\right)<$ $\tau\left(i_{j+1}\right)$ for any $j \in[k-1]$. Note that by the definition of $X_{i}$, we have $\left|I_{i}\right|=X_{i}$. Define

$$
I_{i}^{\prime}:=I_{i} \backslash\left(E_{i} \cup F_{i} \cup E_{i}^{\prime} \cup F_{i}^{\prime}\right)
$$

In other words, $I_{i}^{\prime}$ is obtained by delete those indices in $E_{i} \cup F_{i} \cup E_{i}^{\prime} \cup F_{i}^{\prime}$ from $I_{i}$ without changing the ordering of the remaining indices in $I_{i}$. The definitions of $B_{i}, E_{i}, F_{i}, E_{i}^{\prime}$ and $F_{i}^{\prime}$ imply that the concatenation of $\left\{I_{i}^{\prime}\right\}_{i \in[m]}$ is a set of indices along which defines an increasing subsequence of $\pi$ and $\tau$. To see this, suppose $j, k$ come from the same $I_{i}^{\prime}$ with $j$ comes before $k$ in $I_{i}^{\prime}$, then by the definition of $I_{i}^{\prime}$ we have $\pi(j)<\pi(k)$ and $\tau(j)<\tau(k)$. On the other hand, suppose $j \in I_{i_{1}}^{\prime}$ and $k \in I_{i_{2}}^{\prime}$ with $i_{1}<i_{2}$. By the definition of $E_{i_{1}}, F_{i_{2}}$, we have

$$
p_{k}(k) \leq \frac{a}{1-q}<p_{\max B_{i_{1}}}(j) \leq p_{k}(j)
$$


which implies that $p_{n}(k)<p_{n}(j)$, thus $\pi(k)>\pi(j) . \tau(k)>\tau(j)$ follows from the similar argument. Hence

$$
\operatorname{LIS}(\pi, \tau) \geq \sum_{i=1}^{m}\left|I_{i}^{\prime}\right|
$$

Moreover, the definitions of $I_{i}$ and $I_{i}^{\prime}$ imply that

$$
X_{i}=\left|I_{i}\right| \leq\left|I_{i}^{\prime}\right|+\sum_{A \in\left\{E_{i}, E_{i}^{\prime}, F_{i}, F_{i}^{\prime}\right\}} \operatorname{LIS}\left(\pi_{A}, \tau_{A}\right),
$$

for $1 \leq i \leq m$. From (4.15) and (4.16), we have

$$
\operatorname{LIS}(\pi, \tau) \geq \sum_{i=1}^{m} X_{i}-\sum_{i=1}^{m} \sum_{A \in\left\{E_{i}, E_{i}^{\prime}, F_{i}, F_{i}^{\prime}\right\}} \operatorname{LIS}\left(\pi_{A}, \tau_{A}\right) .
$$

Thus from (4.12) and (4.17), we get

$$
\mathbb{E}\left(\left|\operatorname{LIS}(\pi, \tau)-\sum_{i=1}^{m} X_{i}\right|\right) \leq \sum_{i=1}^{m} \sum_{A \in\left\{E_{i}, E_{i}^{\prime}, F_{i}, F_{i}^{\prime}\right\}} \mathbb{E}\left(\operatorname{LIS}\left(\pi_{A}, \tau_{A}\right)\right)+\mathbb{E}\left(X_{m+1}\right) .
$$

Therefore, (4.10) is a direct consequence of the next lemma.

\section{Lemma 4.2.1.}

$$
\begin{gathered}
\varlimsup_{\beta \rightarrow \infty} \varlimsup_{q \rightarrow 1} \mathbb{E}\left(\frac{X_{m+1}}{n \sqrt{1-q}}\right)=0, \\
\varlimsup_{\beta \rightarrow \infty} \varlimsup_{q \rightarrow 1} \frac{\sum_{i=1}^{m} \mathbb{E}\left(\operatorname{LIS}\left(\pi_{A_{i}}, \tau_{A_{i}}\right)\right)}{n \sqrt{1-q}}=0,
\end{gathered}
$$

for $A_{i} \in\left\{E_{i}, E_{i}^{\prime}, F_{i}, F_{i}^{\prime}\right\}$.

Before proving Lemma 4.2.1, we state the following technical lemma whose proof will be presented at the end of this section. Both Lemma 4.2.2 and Lemma 3.2.11 will be used to reduce the claim in Lemma 4.2.1 to the result of Lemma 5.1 in [6].

Lemma 4.2.2. Given consecutive indices $B \subset[n], 0<q<1$ and any constant $C>0$, there exists a coupling of $q$-Mallows processes $\left\{p_{i}\right\},\left\{p_{i}^{\prime}\right\}$ and $\left\{\hat{p}_{i}\right\}$ such that

- $\left\{p_{i}\right\}$ and $\left\{p_{i}^{\prime}\right\}$ are independent. 
- Let $\pi, \tau$ be as defined in (4.13). Define $\hat{\pi}(j):=n+1-\hat{p}_{n}(j)$ and

$$
F:=\left\{j \in B: p_{j}(j)>C\right\}, \quad \hat{F}:=\left\{j \in B: \hat{p}_{j}(j)>C\right\}
$$

Then, we have $F=\hat{F}$ and $\operatorname{LIS}\left(\pi_{F}, \tau_{F}\right) \leq \operatorname{LIS}\left(\hat{\pi}_{F}\right)$.

Proof of Lemma 4.2.1. To prove (4.19), Define $X:=\operatorname{LIS}\left(\pi_{B_{m+1}}\right)$. By Lemma 3.2.11, letting $\boldsymbol{a}=B_{m+1}$, we have

$$
\mathbb{E}\left(X_{m+1}\right)=\mathbb{E}\left(\operatorname{LIS}\left(\pi_{B_{m+1}}, \tau_{B_{m+1}}\right)\right) \leq \mathbb{E}(X),
$$

and (4.19) follows from the first equation in Lemma 5.1 in [6].

To prove (4.20), by symmetry, we only need to show (4.20) holds when $A_{i}=$ $E_{i}, F_{i}$. For the case when $A_{i}=E_{i}$, define

$$
I:=\left(1,2, \ldots,\left\lfloor\frac{a}{1-q}\right\rfloor\right), \quad \sigma:=p_{\max B_{i}}, \quad \bar{E}_{i}:=\sigma^{-1}(I) .
$$

We have

$$
\begin{aligned}
\operatorname{LIS}\left(\pi_{E_{i}}, \tau_{E_{i}}\right) \leq \operatorname{LIS}\left(\pi_{\bar{E}_{i}}, \tau_{\bar{E}_{i}}\right) & =\operatorname{LIS}\left(\left(p_{n}\right)_{\bar{E}_{i}},\left(p_{n}^{\prime}\right)_{\bar{E}_{i}}\right) \\
& =\operatorname{LIS}\left(\sigma_{\bar{E}_{i}},\left(p_{\max B_{i}}^{\prime}\right)_{\bar{E}_{i}}\right) \\
& =\operatorname{LIS}\left(\left(\sigma_{\bar{E}_{i}}\right)^{r},\left(\left(p_{\max B_{i}}^{\prime}\right)_{\bar{E}_{i}}\right)^{r}\right) .
\end{aligned}
$$

By Lemma 1.1.6 and (4.21), conditioned on the value of $\bar{E}_{i}$, we have $\sigma_{\bar{E}_{i}} \sim \mu_{\left\lfloor\frac{a}{1-q}\right\rfloor, 1 / q}$. By Lemma 1.1.4, we have $\left(\sigma_{\bar{E}_{i}}\right)^{r} \sim \mu_{\left\lfloor\frac{a}{1-q}\right\rfloor, q}$. Moreover, conditioned on the value of $\bar{E}_{i}$, $\left(\sigma_{\bar{E}_{i}}\right)^{r}$ and $\left(\left(p_{\max B_{i}}^{\prime}\right)_{\bar{E}_{i}}\right)^{r}$ are independent. Thus, by choosing $\boldsymbol{a}=I$ in Lemma 3.2.11, there exists a random variable $Z$ with $Z \sim \mu_{\left\lfloor\frac{a}{1-q}\right\rfloor, q}$ such that

$$
\operatorname{LIS}\left(\left(\sigma_{\bar{E}_{i}}\right)^{r},\left(\left(p_{\max B_{i}}^{\prime}\right)_{\bar{E}_{i}}\right)^{r}\right) \leq \operatorname{LIS}(Z)
$$

Hence it follows from (4.22) that $\operatorname{LIS}\left(\pi_{E_{i}}, \tau_{E_{i}}\right) \leq \operatorname{LIS}(Z)$. For any $a>5$, since $0<q<$ 1 , we have $\left\lfloor\frac{a}{1-q}\right\rfloor>5$. Thus

$$
1-\frac{4}{\left\lfloor\frac{a}{1-q}\right\rfloor} \geq 1-\frac{5}{\frac{a}{1-q}}>q .
$$


Hence, by Theorem 1.3 in [6], there exists a constant $c$ such that

$$
\mathbb{E}\left(\operatorname{LIS}\left(\pi_{E_{i}}, \tau_{E_{i}}\right)\right) \leq \mathbb{E}(\operatorname{LIS}(Z)) \leq c\left\lfloor\frac{a}{1-q}\right\rfloor \sqrt{1-q} \leq \frac{c a}{\sqrt{1-q}} .
$$

Hence, from the definition of $m$ in (4.9) and the property of $a$ as defined in (4.14), it follows that

$$
\begin{aligned}
& \varlimsup_{\beta \rightarrow \infty} \varlimsup_{q \rightarrow 1} \frac{\sum_{i=1}^{m} \mathbb{E}\left(\operatorname{LIS}\left(\pi_{E_{i}}, \tau_{E_{i}}\right)\right)}{n \sqrt{1-q}} \leq \varlimsup_{\beta \rightarrow \infty} \varlimsup_{q \rightarrow 1} \frac{m c a}{n(1-q)} \\
\leq & \varlimsup_{\beta \rightarrow \infty} \varlimsup_{q \rightarrow 1} \frac{c a}{\beta(q)}=\varlimsup_{\beta \rightarrow \infty} \frac{c a}{\beta}=0,
\end{aligned}
$$

which completes the proof of (4.20) when $A_{i}=E_{i}$. For the case when $A_{i}=F_{i}$, by Lemma 4.2.2, there exists a coupling such that

$$
\mathbb{E}\left(\operatorname{LIS}\left(\pi_{F_{i}}, \tau_{F_{i}}\right)\right) \leq \mathbb{E}\left(\operatorname{LIS}\left(\hat{\pi}_{F_{i}}\right)\right) .
$$

The claim follows directly from the third equation in Lemma 5.1 in [6].

Next we establish (4.11), which combined with (4.10) imply (4.8), whence completes the proof of Theorem 5. We will make use of Theorem 3, specifically Corollary 3. Define

$$
\bar{J}(\beta)=\sqrt{\frac{\beta}{6 \sinh (\beta / 2)}} \cdot \int_{0}^{1} \sqrt{\cosh (\beta / 2)+2 \cosh (\beta[2 x-1] / 2)} d x .
$$

First we show that

$$
\lim _{\beta \rightarrow \infty} \frac{\bar{J}(\beta)}{\sqrt{\beta}}=\frac{1}{\sqrt{6}} .
$$

Since $\lim _{x \rightarrow \infty} \operatorname{coth}(x)=1$, by (4.24), it suffices to show

$$
\lim _{\beta \rightarrow \infty} \int_{0}^{1} \sqrt{1+2 \cosh (\beta[2 x-1] / 2) / \cosh (\beta / 2)} d x=1 .
$$

Note that

$$
\begin{gathered}
1+2 \cdot \frac{\cosh (\beta[2 x-1] / 2)}{\cosh (\beta / 2)} \\
=1+2 \cdot \frac{e^{\beta(2 x-1) / 2}+e^{-\beta(2 x-1) / 2}}{e^{\beta / 2}+e^{-\beta / 2}}
\end{gathered}
$$




$$
=1+2 \cdot \frac{e^{\beta(x-1)}+e^{-\beta x}}{1+e^{-\beta}}<1+2(1+1)=5 .
$$

for any $x \in[0,1]$ and $\beta>0$. Hence, by dominated convergence theorem, we have

$$
\begin{aligned}
& \lim _{\beta \rightarrow \infty} \int_{0}^{1} \sqrt{1+2 \cosh (\beta[2 x-1] / 2) / \cosh (\beta / 2)} d x \\
= & \int_{0}^{1} \lim _{\beta \rightarrow \infty} \sqrt{1+2 \cosh (\beta[2 x-1] / 2) / \cosh (\beta / 2)} d x \\
= & \int_{0}^{1} 1 d x=1 .
\end{aligned}
$$

(4.26) as well as (4.25) follow.

We continue with the notation defined in Section 4.2. Suppose $n=n(q)$ is such that (4.7) holds. Recall that $X_{1}$ denotes the length of the LIS of two independent Mallows permutations with the same distribution $\mu_{\frac{\beta(q)}{(1-q)}, q}$. Since

$$
\lim _{q \rightarrow 1} \frac{\beta(q)}{1-q} \cdot(1-q)=\beta,
$$

we can apply Corollary 3 and Corollary 3.1.4 to $X_{1}$ and deduce that

$$
\sqrt{\frac{1-q}{\beta(q)}} \cdot X_{1} \stackrel{p}{\longrightarrow} 2 \bar{J}(\beta) .
$$

Now fix $\beta_{0}$ sufficiently large and $q_{0}$ sufficiently close to 1 such that $\beta>\beta_{0}$ and $q_{0} \leq$ $q<1$ imply $\frac{1}{2}<q<1-\frac{4(1-q)}{\beta(q)}$. By (68) in [6] and Lemma 3.2.11, it follows that

$$
\left\{\left(\frac{\sqrt{1-q}}{\beta(q)} \cdot X_{1}\right)^{2}\right\} \text { indexed by } q_{0}<q<1 \text { are uniformly integrable. }
$$

Since $\beta(q) \rightarrow \beta$ as $q \rightarrow 1,(4.27)$ and (4.28) imply that for any fixed $\beta>\beta_{0}$,

$$
\sqrt{\frac{1-q}{\beta}} \cdot X_{1} \stackrel{L_{2}}{\longrightarrow} 2 \bar{J}(\beta)
$$

as $q \rightarrow 1$. Hence, for any fixed $\beta>\beta_{0}$, we have

$$
\lim _{q \rightarrow 1} \sqrt{\frac{1-q}{\beta}} \cdot \mathbb{E}\left(X_{1}\right)=2 \bar{J}(\beta) \quad \text { and } \quad \lim _{q \rightarrow 1}(1-q) \cdot \operatorname{Var}\left(X_{1}\right)=0 .
$$

Let $Y:=\frac{\sum_{i=1}^{m} X_{i}}{n \sqrt{1-q}}$. To prove $(4.11)$, we first show that

$$
\lim _{\beta \rightarrow \infty} \lim _{q \rightarrow 1} \mathbb{E}(Y)=\frac{\sqrt{6}}{3},
$$




$$
\lim _{\beta \rightarrow \infty} \lim _{q \rightarrow 1} \operatorname{Var}(Y)=0
$$

To prove (4.30), note that since $\left\{X_{i}\right\}_{i \in[m]}$ are i.i.d. random variables, we have

$$
\mathbb{E}(Y)=\frac{m}{n \sqrt{1-q}} \mathbb{E}\left(X_{1}\right)=\frac{m \beta}{n(1-q)} \cdot \frac{\sqrt{1-q}}{\beta} \cdot \mathbb{E}\left(X_{1}\right) .
$$

By the definition of $m$ and (4.7), we have

$$
\lim _{q \rightarrow 1} \frac{m \beta}{n(1-q)}=1
$$

Hence, from (4.32) and using (4.29), it follows that

$$
\lim _{q \rightarrow 1} \mathbb{E}(Y)=\frac{1}{\sqrt{\beta}} \cdot \lim _{q \rightarrow 1} \sqrt{\frac{1-q}{\beta}} \cdot \mathbb{E}\left(X_{1}\right)=\frac{2 \bar{J}(\beta)}{\sqrt{\beta}} .
$$

Thus, (4.30) follows from (4.25), since

$$
\lim _{\beta \rightarrow \infty} \lim _{q \rightarrow 1} \mathbb{E}(Y)=\lim _{\beta \rightarrow \infty} \frac{2 \bar{J}(\beta)}{\sqrt{\beta}}=\frac{\sqrt{6}}{3} .
$$

To prove (4.31), again since $\left\{X_{i}\right\}_{i \in[m]}$ are i.i.d., by (4.33), we have

$$
\begin{aligned}
\lim _{q \rightarrow 1} \operatorname{Var}(Y) & =\lim _{q \rightarrow 1} \frac{m}{n^{2}(1-q)} \operatorname{Var}\left(X_{1}\right) \\
& =\lim _{q \rightarrow 1} \frac{1}{\beta n} \operatorname{Var}\left(X_{1}\right)=\lim _{q \rightarrow 1} \frac{1}{\beta n(1-q)}(1-q) \operatorname{Var}\left(X_{1}\right) .
\end{aligned}
$$

Hence, for $\beta>\beta_{0}$, (4.7) and (4.29) imply that

$$
\lim _{q \rightarrow 1} \operatorname{Var}(Y)=0
$$

proving (4.31). Finally, by the triangle and Jensen's inequalities we have

$$
\mathbb{E}\left|Y-\frac{\sqrt{6}}{3}\right| \leq \mathbb{E}|Y-\mathbb{E}(Y)|+\left|\mathbb{E}(Y)-\frac{\sqrt{6}}{3}\right| \leq \sqrt{\operatorname{Var}(Y)}+\left|\mathbb{E}(Y)-\frac{\sqrt{6}}{3}\right|
$$

which shows that (4.30) and (4.31) imply (4.11).

\subsection{Proof of Lemma 4.2.2}

Let $M:=\max \{i \in B\}$. Let $\left\{p_{i}\right\}$ be a $q$-Mallows process. Given a constant $C$, recall that we define $F:=\left\{i \in B: p_{i}(i)>C\right\}$. First we will prove the following claim. 
Claim 1: Given increasing indices $\boldsymbol{v}=\left(v_{1}, v_{2}, \ldots, v_{l}\right)$ with $v_{i} \in B$, for any $1 \leq j<k \leq l$ and any permutation $\boldsymbol{b}=\left(b_{1}, b_{2}, \ldots, b_{M}\right) \in S_{M}$ with $b_{v_{j}}<b_{v_{k}}$, we have

$$
\mathbb{P}\left(p_{M}=\boldsymbol{b} \mid F=\boldsymbol{v}\right) \leq \mathbb{P}\left(p_{M}=\boldsymbol{b} \circ\left(v_{j}, v_{k}\right) \mid F=\boldsymbol{v}\right)
$$

Here $\boldsymbol{b} \circ\left(v_{j}, v_{k}\right)$ denotes the permutation obtained by switching $b_{v_{j}}$ and $b_{v_{k}}$ in $\boldsymbol{b}$.

Proof of Claim 1. If $\mathbb{P}\left(p_{M}=\boldsymbol{b} \mid F_{i}=\boldsymbol{v}\right)=0$, the claim holds trivially. Suppose $\mathbb{P}\left(p_{M}=\boldsymbol{b} \mid F_{i}=\boldsymbol{v}\right)>0$, i.e. there exists $\boldsymbol{t}=\left(t_{1}, \ldots, t_{M}\right)$ such that

(i) $1 \leq t_{i} \leq i$

(ii) if $i \in B, t_{i}>C$ if only if $i \in \boldsymbol{v}$,

(iii) if $p_{i}(i)=t_{i}$ for $i \in[M]$, we have $p_{M}=\boldsymbol{b}$.

Define

$$
\left\{\begin{array}{l}
\hat{t}_{i}:=t_{i} \quad \text { if } 1 \leq i<v_{j} \text { or } v_{k}<i \leq M \\
\hat{t}_{i}:=t_{i}-\mathbb{1}\left(b_{v_{j}}<p_{M}(i)<b_{v_{k}}\right) \quad \text { if } v_{j}<i<v_{k} ; \\
\hat{t}_{v_{j}}:=v_{j}-\sum_{i=1}^{v_{j}} \mathbb{1}\left(p_{M}(i)>b_{v_{k}}\right) ; \\
\hat{t}_{v_{k}}:=v_{k}-\sum_{i=1}^{v_{k}} \mathbb{1}\left(p_{M}(i)>b_{v_{j}}\right) .
\end{array}\right.
$$

We show that, if at each step of the $q$-Mallows process $\left\{\hat{p}_{i}\right\}$,

$$
\hat{p}_{i}(i)=\hat{t}_{i} \quad \text { for any } i \in[M]
$$

we have $\hat{p}_{M}=\boldsymbol{b} \circ\left(v_{j}, v_{k}\right)$. Moreover, if we define $\hat{F}:=\left\{i \in B: \hat{p}_{i}(i)>C\right\}$, then $F=\hat{F}$.

We first show that $\hat{t}_{v_{i}}$ as defined in (4.35) satisfy that $C<\hat{t}_{v_{i}} \leq v_{i}$. We will prove this claim in different cases depending on the value of $i$.

- For $1 \leq i<j$ or $k<i \leq l$, we have $\hat{t}_{v_{i}}=t_{v_{i}}$. Thus by (i), it follows that $C<\hat{t}_{v_{i}} \leq v_{i}$

- For $j<i<k$, we have

$$
\hat{t}_{v_{i}} \leq t_{v_{i}} \leq v_{i}
$$


On the other hand, by the definition of Mallows process, $p_{M}\left(v_{i}\right)>b_{v_{j}}$ if and only if $p_{v_{i}}\left(v_{i}\right)>p_{v_{i}}\left(v_{j}\right)$. Hence if $\mathbb{1}\left(b_{v_{j}}<p_{M}\left(v_{i}\right)<b_{v_{k}}\right)=1$, we have

$$
t_{v_{i}}=p_{v_{i}}\left(v_{i}\right)>p_{v_{i}}\left(v_{j}\right) \geq t_{v_{j}}>C,
$$

which means $\mathbb{1}\left(b_{v_{j}}<p_{M}\left(v_{i}\right)<b_{v_{k}}\right)=1$ implies $t_{v_{i}}>C+1$. Thus

$$
\hat{t}_{v_{i}}=t_{v_{i}}-\mathbb{1}\left(b_{v_{j}}<p_{M}\left(v_{i}\right)<b_{v_{k}}\right)>C .
$$

- To show $C<\hat{t}_{v_{j}} \leq v_{j}$, note that by the definition of $\hat{t}_{v_{j}}$ in (4.35), we have $\hat{t}_{v_{j}} \leq v_{j}$. To show $\hat{t}_{v_{j}}>C$, note that since $p_{v_{j}}$ is a permutation in $S_{v_{j}}$, we have

$$
\begin{aligned}
v_{j}-t_{v_{j}} & =\sum_{i=1}^{v_{j}} \mathbb{1}\left(p_{v_{j}}(i)>t_{v_{j}}\right) \\
& =\sum_{i=1}^{v_{j}} \mathbb{1}\left(p_{M}(i)>b_{v_{j}}\right) \geq \sum_{i=1}^{v_{j}} \mathbb{1}\left(p_{M}(i)>b_{v_{k}}\right) .
\end{aligned}
$$

Here the last inequality follows since $b_{v_{j}}<b_{v_{k}}$. The definition of $\hat{t}_{v_{j}}$ and (4.37) imply $\hat{t}_{v_{j}} \geq t_{v_{j}}>C$.

- To show $C<\hat{t}_{v_{k}} \leq v_{k}$, again by the definition of $\hat{t}_{v_{k}}$ in (4.35), we have $\hat{t}_{v_{k}} \leq v_{k}$. To show $\hat{t}_{v_{k}}>C$, note that since $p_{v_{k}}$ is a permutation in $S_{v_{k}}$, we have

$$
\begin{aligned}
v_{k}-t_{v_{j}} & =\sum_{i=1}^{v_{k}} \mathbb{1}\left(p_{v_{k}}(i)>t_{v_{j}}\right) \\
& \geq \sum_{i=1}^{v_{k}} \mathbb{1}\left(p_{v_{k}}(i)>p_{v_{k}}\left(v_{j}\right)\right)=\sum_{i=1}^{v_{k}} \mathbb{1}\left(p_{M}(i)>b_{v_{j}}\right) .
\end{aligned}
$$

Here the inequality follows since $t_{v_{j}}=p_{v_{j}}\left(v_{j}\right) \leq p_{v_{k}}\left(v_{j}\right)$. The definition of $\hat{t}_{v_{k}}$ and (4.38) imply $\hat{t}_{v_{k}} \geq t_{v_{j}}>C$

For $i \in B \backslash \boldsymbol{v}$, by the definition of $\hat{t}_{i}$, we have $\hat{t}_{i} \leq C$. For $v_{j}<i<v_{k}$, $\mathbb{1}\left(b_{v_{j}}<p_{M}(i)<b_{v_{k}}\right)=1$ implies $t_{i}=p_{i}(i)>p_{i}\left(v_{j}\right) \geq 1$. Hence

$$
\hat{t}_{i}=t_{i}-\mathbb{1}\left(b_{v_{j}}<p_{M}(i)<b_{v_{k}}\right) \geq 1 .
$$


Therefore, we have shown $F=\hat{F}$. The claim that $\hat{p}_{i}(i)=\hat{t}_{i}$ at every step $i \in[M]$ will result to $\hat{p}_{M}=\boldsymbol{b} \circ\left(v_{j}, v_{k}\right)$ can be proved by induction. The induction is taken in reverse order with the base case $i=M$ and the induction step is established by using the second part of Lemma 4.1.4 and the definition of $\hat{t}_{i}$. Here we omit the proof.

To prove the Claim 1, note that conditioned on $F=\boldsymbol{v}$, the random variables $p_{i}(i)$ are still independent with truncated geometric distribution. Hence, we have

$$
\begin{aligned}
& \mathbb{P}\left(\left\{p_{i}(i)=t_{i}: i \in[M]\right\} \mid F=\boldsymbol{v}\right)=c \cdot q^{\sum_{i=1}^{M} t_{i}}, \\
& \mathbb{P}\left(\left\{p_{i}(i)=\hat{t}_{i}: i \in[M]\right\} \mid F=\boldsymbol{v}\right)=c \cdot q^{\sum_{i=1}^{M} \hat{t}_{i}},
\end{aligned}
$$

Here $c$ is a normalizing constant. By Corollary 4.1.3, we have

$$
\sum_{i=1}^{M} t_{i}=\frac{(M+1) M}{2}-l(\boldsymbol{b}), \quad \sum_{i=1}^{M} \hat{t}_{i}=\frac{(M+1) M}{2}-l\left(\boldsymbol{b} \circ\left(v_{j}, v_{k}\right)\right) .
$$

Since $b_{v_{j}}<b_{v_{k}}$ implies $l(\boldsymbol{b})<l\left(\boldsymbol{b} \circ\left(v_{j}, v_{k}\right)\right)$, we have $\sum_{i=1}^{M} t_{i}>\sum_{i=1}^{M} \hat{t}_{i}$. Thus, by (4.39) and (4.40),

$$
\mathbb{P}\left(\left\{p_{i}(i)=t_{i}: i \in[M]\right\} \mid F=\boldsymbol{v}\right)<\mathbb{P}\left(\left\{p_{i}(i)=\hat{t}_{i}: i \in[M]\right\} \mid F=\boldsymbol{v}\right) .
$$

By (iii) and (4.36), Claim 1 follows.

Based on Claim 1, we next prove the following claim.

Claim 2: For any $\kappa \in S_{M}$ and any $w \in[M-1]$ such that $\kappa^{-1}(w)<\kappa^{-1}(w+1)$, there exists a coupling of two $q$-Mallows process $\left\{\bar{p}_{i}\right\}$ and $\left\{\hat{p}_{i}\right\}$ such that the following are satisfied.

- With $\bar{F}:=\left\{i \in B: \bar{p}_{i}(i)>C\right\}$ and $\hat{F}:=\left\{i \in B: \hat{p}_{i}(i)>C\right\}$, we have $\bar{F}=\hat{F}$.

- $\operatorname{LIS}\left(\left(\bar{p}_{M}\right)_{F}, \kappa_{F}\right) \leq \operatorname{LIS}\left(\left(\hat{p}_{M}\right)_{F},((w, w+1) \circ \kappa)_{F}\right)$.

Proof of Claim 2. By Lemma 4.1.2, we know that the values of $\left\{p_{i}(i)\right\}_{i \in[M]}$ is determined by $p_{M}$. Hence, to construct a coupling of $\left\{\bar{p}_{i}\right\}$ and $\left\{\hat{p}_{i}\right\}$, it suffices to define a coupling of $\left(\bar{p}_{M}, \hat{p}_{M}\right)$. 
Let $\left\{p_{i}\right\}$ be a $q$-Mallows process. Define $F:=\left\{i \in B: p_{i}(i)>C\right\}$. Let $\boldsymbol{v}=$ $\left\{v_{1}, \cdots, v_{l}\right\}$ be an arbitrary increasing indices in $[M]$. Conditioned on $F=\boldsymbol{v}$, we defined $\left(\bar{p}_{M}, \hat{p}_{M}\right)$ as follows.

- If $\kappa^{-1}(w) \notin \boldsymbol{v}$ or $\kappa^{-1}(w+1) \notin \boldsymbol{v}$, define $\bar{p}_{M}=\hat{p}_{M}=p_{M}$.

- If $\kappa^{-1}(w)=v_{j}$ and $\kappa^{-1}(w+1)=v_{k}$, note that we can partition $S_{M}$ into pairs of permutations $\left\{\boldsymbol{b}, \boldsymbol{b} \circ\left(v_{j}, v_{k}\right)\right\}$ with $b_{v_{j}}<b_{v_{k}}$. Then, first choose a pair of permutation such that $\left\{\boldsymbol{b}, \boldsymbol{b} \circ\left(v_{j}, v_{k}\right)\right\}$ being chosen with probability $\mathbb{P}\left(p_{M}=\boldsymbol{b} \mid F=\boldsymbol{v}\right)+\mathbb{P}\left(p_{M}=\boldsymbol{b} \circ\left(v_{j}, v_{k}\right) \mid F=\boldsymbol{v}\right)$. Then flip a coin with probability of head being

$$
h:=\frac{2 \cdot \mathbb{P}\left(p_{M}=\boldsymbol{b} \mid F=\boldsymbol{v}\right)}{\mathbb{P}\left(p_{M}=\boldsymbol{b} \mid F=\boldsymbol{v}\right)+\mathbb{P}\left(p_{M}=\boldsymbol{b} \circ\left(v_{j}, v_{k}\right) \mid F=\boldsymbol{v}\right)} .
$$

If the outcome is tail, define $\bar{p}_{M}=\hat{p}_{M}=\boldsymbol{b} \circ\left(v_{j}, v_{k}\right)$. If the outcome is head, then, with equal probability, define either $\bar{p}_{M}=\boldsymbol{b}, \hat{p}_{M}=\boldsymbol{b} \circ\left(v_{j}, v_{k}\right)$ or $\bar{p}_{M}=\boldsymbol{b} \circ\left(v_{j}, v_{k}\right)$, $\hat{p}_{M}=\boldsymbol{b}$.

For the first case, note that $\kappa^{-1}(i) \notin \boldsymbol{v}$ or $\kappa^{-1}(i+1) \notin \boldsymbol{v}$ imply $\kappa_{\boldsymbol{v}}=((i, i+1) \circ \kappa)_{\boldsymbol{v}}$. Hence, by setting $\bar{p}_{M}=\hat{p}_{M}=p_{M}$, the two conditions in the claim are satisfied trivially. For the second case, note that by Claim 1, the probability of being head $h$ defined in (4.41) is no greater than 1. Also as shown in the proof of Claim 1, when one of $\bar{p}_{M}$ and $\hat{p}_{M}$ equals $\boldsymbol{b}$ and the other equals $\boldsymbol{b} \circ\left(v_{j}, v_{k}\right)$, we have $\bar{F}=\hat{F}=\boldsymbol{v}$. Moreover, it is easy to verify that $((w, w+1) \circ \kappa)_{\boldsymbol{v}}=\kappa_{\boldsymbol{v}} \circ(j, k)$ and $\left(\boldsymbol{b} \circ\left(v_{j}, v_{k}\right)\right)_{\boldsymbol{v}}=\boldsymbol{b}_{\boldsymbol{v}} \circ(j, k)$. Hence, when the outcome of the coin is head we have

$$
\operatorname{LIS}\left(\left(\bar{p}_{M}\right)_{\boldsymbol{v}}, \kappa_{\boldsymbol{v}}\right)=\operatorname{LIS}\left(\left(\hat{p}_{M}\right)_{\boldsymbol{v}},((w, w+1) \circ \kappa)_{\boldsymbol{v}}\right) .
$$

When is outcome is tail, we need to show that

$$
\operatorname{LIS}\left(\left(\boldsymbol{b} \circ\left(v_{j}, v_{k}\right)\right)_{\boldsymbol{v}}, \kappa_{\boldsymbol{v}}\right) \leq \operatorname{LIS}\left(\left(\boldsymbol{b} \circ\left(v_{j}, v_{k}\right)\right)_{\boldsymbol{v}},((w, w+1) \circ \kappa)_{\boldsymbol{v}}\right) .
$$


Note that we have $\left(\boldsymbol{b} \circ\left(v_{j}, v_{k}\right)\right)_{\boldsymbol{v}}=\boldsymbol{b}_{\boldsymbol{v}} \circ(j, k)$ and $((w, w+1) \circ \kappa)_{\boldsymbol{v}}=(r, r+1) \circ \kappa_{\boldsymbol{v}}$. Here $r$ denote the rank of $w$ among $\kappa$ restricted on $\boldsymbol{v}$. Moreover, we have $\left(\kappa_{\boldsymbol{v}}\right)^{-1}(r)=j<$ $k=\left(\kappa_{\boldsymbol{v}}\right)^{-1}(r+1)$. Hence by Lemma 3.1.3, we have

$$
\begin{aligned}
\operatorname{LIS}\left(\left(\boldsymbol{b} \circ\left(v_{j}, v_{k}\right)\right)_{\boldsymbol{v}}, \kappa_{\boldsymbol{v}}\right) & =\operatorname{LIS}\left(\boldsymbol{b}_{\boldsymbol{v}} \circ(j, k), \kappa_{\boldsymbol{v}}\right) \\
& =\operatorname{LIS}\left(\boldsymbol{b}_{\boldsymbol{v}} \circ(j, k) \circ\left(\kappa_{\boldsymbol{v}}\right)^{-1}, i d\right), \\
\operatorname{LIS}\left(\left(\boldsymbol{b} \circ\left(v_{j}, v_{k}\right)\right)_{\boldsymbol{v}},((w, w+1) \circ \kappa)_{\boldsymbol{v}}\right) & =\operatorname{LIS}\left(\boldsymbol{b}_{\boldsymbol{v}} \circ(j, k),(r, r+1) \circ \kappa_{\boldsymbol{v}}\right) \\
& =\operatorname{LiS}\left(\boldsymbol{b}_{\boldsymbol{v}} \circ(j, k) \circ\left(\kappa_{\boldsymbol{v}}\right)^{-1},(r, r+1)\right) .
\end{aligned}
$$

Here $i d$ denotes the identity in $S_{l}$. Note that

$$
\begin{aligned}
& \boldsymbol{b}_{\boldsymbol{v}} \circ(j, k) \circ\left(\kappa_{\boldsymbol{v}}\right)^{-1}(r)=\boldsymbol{b}_{\boldsymbol{v}} \circ(j, k)(j)=\boldsymbol{b}_{\boldsymbol{v}}(k), \\
& \boldsymbol{b}_{\boldsymbol{v}} \circ(j, k) \circ\left(\kappa_{\boldsymbol{v}}\right)^{-1}(r+1)=\boldsymbol{b}_{\boldsymbol{v}} \circ(j, k)(k)=\boldsymbol{b}_{\boldsymbol{v}}(j) .
\end{aligned}
$$

Since $b_{v_{j}}<b_{v_{k}}$, we have $\boldsymbol{b}_{\boldsymbol{v}}(j)<\boldsymbol{b}_{\boldsymbol{v}}(k)$, which means $\{r, r+1\}$ is a pair of inversion for the permutation $\boldsymbol{b}_{\boldsymbol{v}} \circ(j, k) \circ\left(\kappa_{\boldsymbol{v}}\right)^{-1}$. Hence (4.42) follows from (4.43) and (4.44).

Finally, it can be easily verified that $\bar{p}_{M}$ and $\hat{p}_{M}$ thus defined have the right marginal distribution, i.e. both $\bar{p}_{M}$ and $\hat{p}_{M}$ have the same distribution as $p_{M}$.

Proof of Lemma 4.2.2. Let $i d_{M}^{r}$ denote the reversal of identity in $S_{M}$. Considering the poset $\left(S_{M}, \leq_{L}\right)$, here $\leq_{L}$ denotes the left weak Bruhat order as defined in 3.2.1, we have that $i d_{M}^{r}$ is the maximum element in $\left(S_{M}, \leq_{L}\right)$. Hence for any permutation $\kappa \neq i d_{M}^{r}$, we can find a sequence of permutations $\left\{\kappa_{i}\right\}$ such that

$$
\kappa=\kappa_{0} \leq_{L} \kappa_{1} \leq_{L} \cdots \leq_{L} \kappa_{m}=i d_{M}^{r}
$$

and $\kappa_{i+1}$ covers $\kappa_{i}$, i.e. there exists $w \in[M-1]$ such that $(w, w+1) \circ \kappa_{i}=\kappa_{i+1}$ and $l\left(\kappa_{i+1}\right)=l\left(\kappa_{i}\right)+1$. Note that here $m=\frac{M(M-1)}{2}-l(\kappa)$. Then by Claim 2 and induction on $m$, it can be shown that, for any $\kappa \in S_{M}$, there exists a coupling, denoted by $\mathcal{C}_{\kappa}$, of two $q$-Mallows processes $\left\{\bar{p}_{i}\right\}$ and $\left\{\hat{p}_{i}\right\}$ such that the following are satisfied.

- With $\bar{F}:=\left\{i \in B: \bar{p}_{i}(i)>C\right\}$ and $\hat{F}:=\left\{i \in B: \hat{p}_{i}(i)>C\right\}$, we have $\bar{F}=\hat{F}$. 
- $\operatorname{LIS}\left(\left(\bar{p}_{M}\right)_{F}, \kappa_{F}\right) \leq \operatorname{LIS}\left(\left(\hat{p}_{M}\right)_{F},\left(i d_{M}^{r}\right)_{F}\right)$.

To construct the coupling $\left\{p_{i}\right\},\left\{p_{i}^{\prime}\right\}$ and $\left\{\hat{p}_{i}\right\}$ in Lemma 4.2.2, note that, by Definition 3.1.2, we have

$$
\begin{aligned}
\operatorname{LIS}\left(\pi_{F}, \tau_{F}\right) & =\operatorname{LIS}(\pi(F), \tau(F))=\operatorname{LIS}\left(p_{n}(F), p_{n}^{\prime}(F)\right) \\
& =\operatorname{LIS}\left(p_{M}(F), p_{M}^{\prime}(F)\right)=\operatorname{LiS}\left(\left(p_{M}\right)_{F},\left(p_{M}^{\prime}\right)_{F}\right), \\
\operatorname{LIS}\left(\hat{\pi}_{F}\right) & =\operatorname{LIS}\left(\hat{\pi}(F), i d_{n}(F)\right)=\operatorname{LIS}\left(\hat{p}_{n}(F),\left(i d_{n}^{r}\right)(F)\right) \\
& =\operatorname{LIS}\left(\hat{p}_{M}(F),\left(i d_{M}^{r}\right)(F)\right)=\operatorname{LiS}\left(\left(\hat{p}_{M}\right)_{F},\left(i d_{M}^{r}\right)_{F}\right) .
\end{aligned}
$$

Here $i d_{n}$ denotes the identity in $S_{n}$. Hence by (4.47) and (4.48) we have

$$
\operatorname{LIS}\left(\pi_{F}, \tau_{F}\right) \leq \operatorname{LIS}\left(\hat{\pi}_{F}\right) \Leftrightarrow \operatorname{LIS}\left(\left(p_{M}\right)_{F},\left(p_{M}^{\prime}\right)_{F}\right) \leq \operatorname{LIS}\left(\left(\hat{p}_{M}\right)_{F},\left(i d_{M}^{r}\right)_{F}\right) .
$$

We define the coupling $\left\{p_{i}\right\},\left\{p_{i}^{\prime}\right\}$ and $\left\{\hat{p}_{i}\right\}$ as follows. For any $i>M$, we simply let $p_{i}, p_{i}^{\prime}$ and $\hat{p}_{i}$ be i.i.d. truncated geometric distributed. For $1 \leq i \leq M$, let $p_{M}^{\prime} \sim \mu_{M, q}$. Conditioned on $p_{M}^{\prime}=\kappa$, define $\left\{p_{i}\right\}$ and $\left\{\hat{p}_{i}\right\}$ such that they have joint distribution $\mathcal{C}_{\kappa}$. The lemma follows from (4.49) and the property of $\mathcal{C}_{\kappa}$. 


\section{Chapter 5}

\section{CENTRAL LIMIT THEOREM FOR THE LCS}

In this chapter we prove Theorem 6 . In the first section, we introduce the construction of Mallows permutation from a sequence of i.i.d. geometric random variables, which enables us to decompose the common subsequence of two permutations as the concatenation of common subsequences within disjoint blocks. In the second section, we prove Theorem 6 by using the central limit theorem for the regenerative processes.

\subsection{Bound LCS via Regenerative Process}

\subsubsection{Constructing Mallows Permutations}

For a given parameter $0<q<1$, Gnedin and Olshanski [14] constructed an infinite Mallows permutation with parameter $q$ on $\mathbb{N}$ by an insertion process, which we will refer to as Mallows $(q)$ process. The process is as follows. Given an i.i.d. sequence $\left\{Z_{i}\right\}_{i>=1}$ of Geom $(1-q)$ variables, construct a permutation $\tilde{\Pi}$ of the natural numbers inductively according the following rule: Set $\tilde{\Pi}(1)=Z_{1}$. For $i>1$, set $\tilde{\Pi}(i)=k$ where $k$ is the $Z_{i}$-th number in the increasing order from the set $\mathbb{N} \backslash\{\tilde{\Pi}(j): 1 \leq j<i\}$. For example, suppose that the realizations of the first five independent geometric random variables are $Z_{1}=4, Z_{2}=4, Z_{3}=1, Z_{4}=2, Z_{5}=3$. Then we have $\tilde{\Pi}(1)=4, \tilde{\Pi}(2)=5$, $\tilde{\Pi}(3)=1, \tilde{\Pi}(4)=3$ and $\tilde{\Pi}(5)=7$. We represent the process step-by-step below.

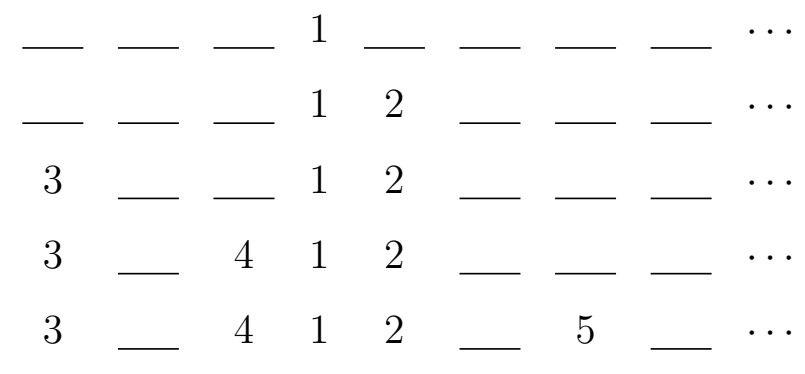


Let $\Pi_{n}$ be the permutation on $[n]$ induced by $\tilde{\Pi}$, i.e.,$\Pi_{n}(i)=j$ if $\tilde{\Pi}(i)$ has rank $j$ when the set $\{\tilde{\Pi}(k): k \in[n]\}$ is arranged in increasing order. Consider the example above when $n=5$. Then we have $\Pi_{5}(1)=3, \Pi_{5}(2)=4, \Pi_{5}(3)=1, \Pi_{5}(4)=2$ and $\Pi_{5}(5)=5$. The following lemma (cf. Lemma 2.1 in [5]) says that $\Pi_{n}$ thus defined is Mallows distributed with parameter $q$.

Lemma 5.1.1. Let $\tilde{\Pi}$ be an infinite Mallows(q) permutation and let $\Pi_{n}$ be the induced permutation on $[n]$ as defined above. Then $\Pi_{n}$ is a Mallows $(q)$ permutation on $[n]$.

\subsubsection{The Regenerative Process Representation}

A stochastic process $\{X(t): t \geq 0\}$ is said to be a regenerative process if there exist regeneration times $0 \leq T_{0}<T_{1}<T_{2}<\cdots$ such that for each $k \geq 1$, the process $\left\{X\left(T_{k}+t\right): t \geq 0\right\}$ has the same distribution as $\left\{X\left(T_{0}+t\right): t \geq 0\right\}$ and is independent of $\left\{X(t): 0 \leq t<T_{k}\right\}$. Below we will define a regenerative process using the Mallows $(q)$ process defined above such that we can bound the LCS of two independent Mallows permutations by the sum of i.i.d. random variables.

Let $\tilde{\Pi}$ and $\tilde{\Pi}^{\prime}$ be two independent infinite Mallows permutations with parameters $q, q^{\prime}$ respectively. Suppose for a given $m \in \mathbb{N}$ we have $\tilde{\Pi}([m])=\tilde{\Pi}^{\prime}([m])=[m]$, i.e. the permutations $\tilde{\Pi}$ and $\tilde{\Pi}^{\prime}$ restricted to $[m]$ defines two bijections from $[m]$ to $[m]$. Define two infinite permutations $\tilde{\Pi}_{m}$ and $\tilde{\Pi}_{m}^{\prime}$ as follows,

$$
\tilde{\Pi}_{m}(i):=\tilde{\Pi}(i+m)-m, \quad \tilde{\Pi}_{m}^{\prime}:=\tilde{\Pi}^{\prime}(i+m)-m, \quad \forall i \in \mathbb{N}
$$

From the construction of $\tilde{\Pi}$ and $\tilde{\Pi}^{\prime}$, it is obvious that $\tilde{\Pi}_{m}$ and $\tilde{\Pi}_{m}^{\prime}$ are also infinite Mallows permutations with parameters $q$ and $q^{\prime}$ respectively. Together with the independence of the geometric variables $\left\{Z_{i}\right\}$ as well as $\left\{Z_{i}^{\prime}\right\}$, it follows that $\left\{\left(\tilde{\Pi}(i)-i, \tilde{\Pi}^{\prime}(i)-i\right)\right\}_{i \in \mathbb{N}}$ is a regenerative process with regeneration times $0=T_{0}<$ $T_{1}<T_{2}<\cdots$ where for $i>1$ we have,

$$
T_{i}:=\min \left\{j>T_{i-1}:\{\tilde{\Pi}(k): k \in[j]\}=\left\{\tilde{\Pi}^{\prime}(k): k \in[j]\right\}=[j]\right\} .
$$


Let $X_{j}:=T_{j}-T_{j-1}$ for $j \geq 1$. Clearly, $X_{j}$ are independent and identically distributed. For $j \geq 1$, define

$$
\Sigma_{j}(i):=\tilde{\Pi}\left(i+T_{j-1}\right)-T_{j-1}, \quad \Sigma_{j}^{\prime}(i):=\tilde{\Pi}^{\prime}\left(i+T_{j-1}\right)-T_{j-1}, \quad \forall i \in\left[X_{j}\right]
$$

Then, both $\Sigma_{j}$ and $\Sigma_{j}^{\prime}$ are permutations of $\left[X_{j}\right]$. Furthermore, the $\left\{\Sigma_{j}\right\}_{j \in \mathbb{N}}$ are i.i.d. and $\left\{\Sigma_{j}^{\prime}\right\}_{j \in \mathbb{N}}$ are i.i.d.. Let $Y_{j}:=\operatorname{LCS}\left(\Sigma_{j}, \Sigma_{j}^{\prime}\right)$ i.e. $Y_{j}$ denotes the length of the longest common subsequence between $\Sigma_{j}$ and $\Sigma_{j}^{\prime}$. Clearly, $\left\{Y_{j}\right\}_{j \in \mathbb{N}}$ are i.i.d.. Then we have the following bounds for the LCS of two independent Mallows permutation.

Lemma 5.1.2. Let $S_{n}:=\min \left\{j: T_{j} \geq n\right\}$. Then we have

$$
\sum_{j=1}^{S_{n}-1} Y_{j}<L C S\left(\Pi_{n}, \Pi_{n}^{\prime}\right) \leq \sum_{j=1}^{S_{n}} Y_{j}
$$

Proof. Given $j>0$, let $\operatorname{LCS}_{\left[T_{j-1}+1, T_{j}\right]}\left(\Pi_{n}, \Pi_{n}^{\prime}\right)$ denote the length of the longest common subsequence of $\Pi_{n}, \Pi_{n}^{\prime}$ restricted on $\left[T_{j-1}+1, T_{j}\right]$. From the definition of $T_{j}$, we have $\Pi_{n}\left(\left[T_{j-1}+1, T_{j}\right]\right)=\Pi_{n}^{\prime}\left(\left[T_{j-1}+1, T_{j}\right]\right)=\left[T_{j-1}+1, T_{j}\right]$. Thus, we get

$$
\sum_{j=1}^{S_{n}-1} \operatorname{LCS}_{\left[T_{j-1}+1, T_{j}\right]}\left(\Pi_{n}, \Pi_{n}^{\prime}\right)<\operatorname{LCS}\left(\Pi_{n}, \Pi_{n}^{\prime}\right) \leq \sum_{j=1}^{S_{n}} \operatorname{LCS}_{\left[T_{j-1}+1, T_{j}\right]}\left(\Pi_{n}, \Pi_{n}^{\prime}\right) .
$$

It follows from the definition of $\Sigma_{j}$ and $\Sigma_{j}^{\prime}$ that there exists a bijection between the common subsequences of $\Pi_{n}, \Pi_{n}^{\prime}$ restricted on $\left[T_{j-1}+1, T_{j}\right]$ and the common subsequences of $\Sigma_{j}, \Sigma_{j}^{\prime}$. Hence we have $\operatorname{LCS}_{\left[T_{j-1}+1, T_{j}\right]}\left(\Pi_{n}, \Pi_{n}^{\prime}\right)=Y_{j}$. The lemma follows.

\subsection{Renewal Time Estimate and the CLT}

In this section, we first prove that the inter-renewal times $X_{i}$ as defined in the previous section has finite first and second moments, which are the conditions required to apply results from the theory of regenerative processes to show Theorem 6 . Again we follow the approach developed in [5], in which the author introduce the following Markov chain.

Let $\left\{M_{n}\right\}_{n \geq 0}$ denote the Markov chain with the state space $\Omega=\mathbb{N} \cup\{0\}$ and the one step transition defined as follows: $M_{n}:=\max \left\{M_{n-1}, Z_{n}\right\}-1$ where $\left\{Z_{i}\right\}$ is 
a sequence of i.i.d. Geom $(1-q)$ variables. Likewise, for the parameter $q^{\prime}$, we define a Markov chain $\left\{M_{n}^{\prime}\right\}_{n \geq 0}$ in the same fashion, i.e., the one step transition rule is defined by $M_{n}^{\prime}:=\max \left\{M_{n-1}^{\prime}, Z_{n}^{\prime}\right\}-1$ where $\left\{Z_{i}^{\prime}\right\}$ is a sequence of i.i.d. $\operatorname{Geom}\left(1-q^{\prime}\right)$ variables. Let $\left\{M_{n}^{\otimes}\right\}_{n \geq 0}$ denote the product chain of $\left\{M_{n}\right\}$ and $\left\{M_{n}^{\prime}\right\}$. Let $R_{0}^{+}$denote the first return time to $(0,0)$ of this chain, i.e.

$$
R_{0}^{+}:=\min \left\{k>0: M_{k}^{\otimes}=(0,0)\right\} .
$$

Lemma 5.2.1. For the Markov chain $\left\{M_{n}^{\otimes}\right\}$ started at $M_{0}^{\otimes}=(0,0)$, the first return time $R_{0}^{+} \stackrel{d}{=} T_{1}$. In other words, $X_{i}$ has the same distribution as $R_{0}^{+}$.

Proof. We couple the Markov chain $M_{n}^{\otimes}=\left(M_{n}, M_{n}^{\prime}\right)$ with the infinite Mallows permutations $\tilde{\Pi}, \tilde{\Pi}^{\prime}$ with parameters $q$ and $q^{\prime}$ respectively by using the same i.i.d. sequences $\left\{Z_{i}\right\}$ and $\left\{Z_{i}^{\prime}\right\}$ with $Z_{i} \sim \operatorname{Geom}(1-q)$ and $Z_{i}^{\prime} \sim \operatorname{Geom}\left(1-q^{\prime}\right)$. Under this coupling, it is easy to verify that

$$
M_{n}=\max _{1 \leq j \leq n}\{\tilde{\Pi}(j)\}-n, \quad M_{n}^{\prime}=\max _{1 \leq j \leq n}\left\{\tilde{\Pi}^{\prime}(j)\right\}-n
$$

The lemma follows from the definition of $T_{1}$ and $R_{0}^{+}$.

We analyze the Markov chain $M_{n}^{\otimes}$ and the first return time $R_{0}^{+}$in the next few lemmas.

Lemma 5.2.2. The Markov chain $M_{n}^{\otimes}$ is a positive recurrent Markov chain with unique stationary distribution $\nu=\left(\nu_{i, j}\right)_{i, j \geq 0}$ where

$$
\nu_{i, j}:=\frac{q^{i}}{\mathcal{Z}(q) \prod_{k=1}^{i}\left(1-q^{k}\right)} \cdot \frac{\left(q^{\prime}\right)^{j}}{\mathcal{Z}\left(q^{\prime}\right) \prod_{k=1}^{j}\left(1-\left(q^{\prime}\right)^{k}\right)} .
$$

Here $\mathcal{Z}(q):=1 / \prod_{k=1}^{\infty}\left(1-q^{k}\right)$.

Note that $\mathcal{Z}(q)$ is finite since $\lim _{k \rightarrow \infty} \log \left(\frac{1}{1-q^{k}}\right) / q^{k}=1$.

Proof. The claim follows directly from Lemma 4.2 in [5] and the fact that $M_{n}^{\otimes}$ is the product chain of $M_{n}$ and $M_{n}^{\prime}$. 
Let $R_{t}$ denote the first time the chain $M_{n}^{\otimes}$ to reach a state $(i, j)$ such that $i+j \leq t$. In the following, we shall denote by $\mathbb{E}_{i, j}$ the expectation with respect to the chain started at the state $(i, j)$ and $\mathbb{E}_{\nu}$ denote the expectation with respect to the chain started from stationary distribution.

Lemma 5.2.3. For any $i, j \geq 0$ with $i+j>0$, we have

$$
\mathbb{E}_{i, j} R_{i+j-1} \geq \mathbb{E}_{i, j+1} R_{i+j}, \quad \mathbb{E}_{i, j} R_{i+j-1} \geq \mathbb{E}_{i+1, j} R_{i+j}
$$

Proof. By symmetry of the two entries $M_{n}$ and $M_{n}^{\prime}$ in $M_{n}^{\otimes}$, it suffices to show the first inequality. We couple two chains $\left(M_{n}, M_{n}^{\prime}\right)$ and $\left(\tilde{M}_{n}, \tilde{M}_{n}^{\prime}\right)$ which start from $(i, j)$ and $(i, j+1)$ respectively by using the same sequences $\left\{Z_{i}\right\}$ and $\left\{Z_{i}^{\prime}\right\}$. It is easily seen from the one step transition rule that, at any time $n$, we have $M_{n}=\tilde{M}_{n}$ and $0 \leq \tilde{M}_{n}^{\prime}-M_{n}^{\prime} \leq 1$. Thus we have

$$
0 \leq\left(\tilde{M}_{n}+\tilde{M}_{n}^{\prime}\right)-\left(M_{n}+M_{n}^{\prime}\right) \leq 1, \quad \forall n \geq 0
$$

Therefore, $M_{n}+M_{n}^{\prime} \leq i+j-1$ implies $\tilde{M}_{n}+\tilde{M}_{n}^{\prime} \leq i+j$.

As the immediate corollary of Lemma 5.2.3, we have

Corollary 5.2.4. For any $i, j \geq 0$ with $i+j>0$,

$$
\max \left\{\mathbb{E}_{0,1} R_{0}, \mathbb{E}_{1,0} R_{0}\right\} \geq \mathbb{E}_{i, j} R_{i+j-1}
$$

The positive recurrence of the chain $M_{n}^{\otimes}$ implies that $\mathbb{E}_{0,1} R_{0}$ and $\mathbb{E}_{1,0} R_{0}$ are finite. Let $\eta:=\max \left\{\mathbb{E}_{0,1} R_{0}, \mathbb{E}_{1,0} R_{0}\right\}$.

Lemma 5.2.5. For any $i, j \geq 0$ with $i+j>0$, we have

$$
\mathbb{E}_{i, j} R_{0} \leq(i+j) \eta
$$

Proof. We proof this lemma by induction on the sum of $i$ and $j$. When $i+j=1$, the claim holds trivially. Suppose the claim holds for any $\{i, j \geq 0: i+j \leq k\}$. Given $s, t$ with $s+t=k+1$, by the Markov property, we have

$$
\mathbb{E}_{s, t} R_{0}=\sum_{n \geq 1} \sum_{i+j \leq k}\left(n+\mathbb{E}_{i, j} R_{0}\right) \cdot \mathbb{P}_{s, t}\left(R_{k}=n, M_{n}^{\otimes}=(i, j)\right)
$$




$$
\begin{aligned}
& \leq \sum_{n \geq 1} \sum_{i+j \leq k}(n+k \eta) \cdot \mathbb{P}_{s, t}\left(R_{k}=n, M_{n}^{\otimes}=(i, j)\right) \\
& =k \eta+\sum_{n \geq 1} \sum_{i+j \leq k} n \cdot \mathbb{P}_{s, t}\left(R_{k}=n, M_{n}^{\otimes}=(i, j)\right) \\
& =k \eta+\sum_{n \geq 1} n \cdot \mathbb{P}_{s, t}\left(R_{k}=n\right) \\
& =k \eta+\mathbb{E}_{s, t} R_{k} \\
& \leq(k+1) \eta .
\end{aligned}
$$

Here the first inequality follows from induction hypothesis and the last inequality follows from Corollary 5.2.4.

Lemma 5.2.6. For the Markov chain $M_{n}^{\otimes}, \mathbb{E}_{\nu} R_{0}<\infty$.

Proof. Due to Lemma 4.2 in [5], define

$$
\begin{aligned}
\mu_{i} & :=\sum_{j=0}^{\infty} \nu_{i, j}=\frac{q^{i}}{\mathcal{Z}(q) \prod_{k=1}^{i}\left(1-q^{k}\right)}, \\
\mu_{j}^{\prime} & :=\sum_{i=0}^{\infty} \nu_{i, j}=\frac{\left(q^{\prime}\right)^{j}}{\mathcal{Z}\left(q^{\prime}\right) \prod_{k=1}^{j}\left(1-\left(q^{\prime}\right)^{k}\right)} .
\end{aligned}
$$

Note that since $\mu_{i}<q^{i} / \mathcal{Z}(q)^{2}$, we have $\sum_{i=0}^{\infty} i \mu_{i}<\infty$. Similarly we also have $\sum_{j=0}^{\infty} j \mu_{j}^{\prime}<\infty$. Hence, we have

$$
\begin{aligned}
\mathbb{E}_{\nu} R_{0}=\sum_{i, j \geq 0} \nu_{i, j} \mathbb{E}_{i, j} R_{0} & \leq \sum_{i, j \geq 0} \nu_{i, j}(i+j) \eta \\
& =\eta \sum_{i=0}^{\infty} i \mu_{i}+\eta \sum_{j=0}^{\infty} j \mu_{j}^{\prime}<\infty .
\end{aligned}
$$

In the next lemma, we show that the first and second moments of the first return time $R_{0}^{+}$are finite by using Kac's formula.

\section{Lemma 5.2.7.}

$$
\mathbb{E}_{0,0} R_{0}^{+}<\infty, \quad \mathbb{E}_{0,0}\left(R_{0}^{+}\right)^{2}<\infty
$$


Proof. It is a basic fact about Markov chain that $\mathbb{E}_{0,0} R_{0}^{+}=\frac{1}{\nu_{0,0}}$. By lemma 5.2.2 and the remark, we have $\frac{1}{\nu_{0,0}}=\mathcal{Z}(q) \cdot \mathcal{Z}\left(q^{\prime}\right)<\infty$. The finiteness of the second moment of $R_{0}^{+}$follows from Lemma 5.2.6 and Kac's formula (cf. Corollary 2.24 in [2]),

$$
\mathbb{E}_{0,0}\left(R_{0}^{+}\right)^{2}=\frac{2 E_{\nu}\left(R_{0}\right)+1}{\nu_{0,0}}
$$

In the remainder of this section, we complete the proof of Theorem 6 by using the following version of central limit theorem due to Anscombe.

Theorem 5.2.8 (Anscombe's Theorem). Let $\left\{X_{i}\right\}_{i \geq 1}$ be a sequence of i.i.d. random variables with mean 0 and positive, finite variance $\sigma^{2}$. For $n \geq 1$, let $Q_{n}:=\sum_{i=1}^{n} X_{i}$. Suppose $\{N(t), t \geq 0\}$ is a family of positive integer-valued random variables such that for some $0<c<\infty$,

$$
\frac{N(t)}{t} \stackrel{p}{\rightarrow} c \quad \text { as } t \rightarrow \infty
$$

Then,

$$
\frac{Q_{N(t)}}{\sqrt{t}} \stackrel{d}{\longrightarrow} \mathcal{N}\left(0, c \sigma^{2}\right) \quad \text { as } t \rightarrow \infty .
$$

Recall that in section 5.1.2, we define $X_{i}$ to be the inter-renewal times and $S_{n}=\min \left\{j: \sum_{i=1}^{j} X_{i} \geq n\right\}$.

Lemma 5.2.9. For $\nu_{0,0}$ as defined in Lemma 5.2.2,

$$
\frac{S_{n}}{n} \stackrel{a . s .}{\longrightarrow} \nu_{0,0}
$$

Proof. Observer that

$$
\frac{\sum_{j=1}^{S_{n}-1} X_{j}}{S_{n}} \leq \frac{n}{S_{n}} \leq \frac{\sum_{j=1}^{S_{n}} X_{j}}{S_{n}} .
$$

As $n \rightarrow \infty$, by SLLN, both the left and right hand sides of the above inequality converge almost surely to $\nu_{0,0}^{-1}$.

As our last step in preparation for the proof of Theorem 6, we introduce the following basic result (cf. Lemma 5.5 in [5]). 
Lemma 5.2.10. Let $W_{1}, W_{2}, \ldots$ be an i.i.d. sequence of non-negative random variables with $\mathbb{E} W_{i}^{2}<\infty$. Then we have for any constant $c>0$,

$$
\frac{\max _{1 \leq i \leq c n} W_{i}}{\sqrt{n}} \stackrel{p}{\longrightarrow} 0
$$

We inherit the notations defined in section 5.1.2. Let $a:=\nu_{0,0} \mathbb{E}\left(Y_{1}\right)$ and $\delta^{2}:=$ $\operatorname{Var}\left(Y_{1}-a X_{1}\right)$. Since $1 \leq Y_{1} \leq X_{1}$, we have $\left|Y_{1}-a X_{1}\right|<(1+a) X_{1}$. Hence by Lemma 5.2.1 and Lemma 5.2.7, we have $\delta^{2}<\infty$. Trivially, $\delta^{2}>0$ since $Y_{1}$ is clearly not constant. Hence, using Theorem 5.2.8 and Lemma 5.2.9, we can show the following regenerative reversion of central limit theorem.

Theorem 5.2.11 (Regenerative CLT). Let $\left(X_{i}, Y_{i}\right)_{i \geq 1}$ and $S_{n}$ be as defined in section 5.1.2. Let $Q_{S_{n}}:=\sum_{i=1}^{S_{n}} Y_{i}$. Then we have

$$
\frac{Q_{S_{n}}-a n}{\sqrt{n}} \stackrel{d}{\longrightarrow} \mathcal{N}\left(0, \delta^{2} \nu_{0,0}\right)
$$

Proof. Define $\tilde{Q}_{S_{n}}=\sum_{i=1}^{S_{n}} Y_{i}-a X_{i}$. Then, by Theorem 5.2 .8 we have

$$
\frac{\tilde{Q}_{S_{n}}}{\sqrt{n}} \stackrel{d}{\longrightarrow} \mathcal{N}\left(0, \delta^{2} \nu_{0,0}\right) \text {. }
$$

By the definition of $S_{n}$, we have

$$
\tilde{Q}_{S_{n}} \leq Q_{S_{n}}-a n \leq \tilde{Q}_{S_{n}}+a \cdot X_{S_{n}} \leq \tilde{Q}_{S_{n}}+a \cdot \max _{1 \leq i \leq n} X_{i}
$$

Here the last inequality follows since $S_{n} \leq n$. By Lemma 5.2.10, we have

$$
\frac{\max _{1 \leq i \leq n} X_{i}}{\sqrt{n}} \stackrel{p}{\longrightarrow} 0
$$

The theorem follows.

Proof of Theorem 6. It follows from Lemma 5.1.2 that

$$
\frac{Q_{S_{n}}-a n}{\sqrt{n}}-\frac{Y_{S_{n}}}{\sqrt{n}} \leq \frac{\operatorname{LCS}\left(\Pi_{n}, \Pi_{n}^{\prime}\right)-a n}{\sqrt{n}} \leq \frac{Q_{S_{n}}-a n}{\sqrt{n}} .
$$

Since $1 \leq Y_{i} \leq X_{i}$, we have $\mathbb{E}\left(Y_{i}^{2}\right)<\mathbb{E}\left(X_{i}^{2}\right)<\infty$ by Lemma 5.2.7. Hence, by Lemma 5.2 .10 , it follows that

$$
\frac{\max _{1 \leq i \leq n} Y_{i}}{\sqrt{n}} \stackrel{p}{\longrightarrow} 0
$$


Since $S_{n} \leq n$, we have $Y_{S_{n}} \leq \max _{1 \leq i \leq n} Y_{i}$. Thus

$$
\frac{Y_{S_{n}}}{\sqrt{n}} \stackrel{p}{\longrightarrow} 0
$$

Therefore, by setting $\sigma:=\delta \sqrt{\nu_{0,0}}$, it follows from Theorem 5.2.11 that

$$
\frac{\operatorname{LCS}\left(\Pi_{n}, \Pi_{n}^{\prime}\right)-a n}{\sigma \sqrt{n}} \stackrel{d}{\longrightarrow} \mathcal{N}(0,1) .
$$

Theorem 6 follows from (5.3) and Lemma 5.1.1. 


\section{BIBLIOGRAPHY}

[1] James Abello, The weak bruhat order of $s_{-} \sigma$, consistent sets, and catalan numbers, SIAM Journal on Discrete Mathematics 4 (1991), no. 1, 1-16.

[2] David Aldous and Jim Fill, Reversible markov chains and random walks on graphs, 2002.

[3] R.B. Ash and C. Doléans-Dade, Probability and measure theory, Harcourt/Academic Press, 2000.

[4] Jinho Baik, Percy Deift, and Kurt Johansson, On the distribution of the length of the longest increasing subsequence of random permutations, Journal of the American Mathematical Society 12 (1999), no. 4, 1119-1178.

[5] Riddhipratim Basu and Nayantara Bhatnagar, Limit theorems for longest monotone subsequences in random mallows permutations, arXiv preprint arXiv:1601.02003 (2016).

[6] Nayantara Bhatnagar and Ron Peled, Lengths of monotone subsequences in a mallows permutation, Probability Theory and Related Fields 161 (2015), no. 3-4, 719-780.

[7] Václáv Chvatal and David Sankoff, Longest common subsequences of two random sequences, Journal of Applied Probability (1975), 306-315.

[8] Douglas E Critchlow, Metric methods for analyzing partially ranked data, vol. 34, Springer Science \& Business Media, 2012.

[9] Vladimír Dancík, Expected length of longest common subsequences, (1994).

[10] Vlado Dančík and Mike Paterson, Upper bounds for the expected length of a longest common subsequence of two binary sequences, Random Structures \& Algorithms 6 (1995), no. 4, 449-458.

[11] Joseph G Deken, Some limit results for longest common subsequences, Discrete Mathematics 26 (1979), no. 1, 17-31.

[12] Jean-Dominique Deuschel and Ofer Zeitouni, Limiting curves for iid records, The Annals of Probability (1995), 852-878. 
[13] Michael A Fligner and Joseph S Verducci, Probability models and statistical analyses for ranking data, vol. 80, Springer, 1993.

[14] Alexander Gnedin and Grigori Olshanski, The two-sided infinite extension of the mallows model for random permutations, Advances in Applied Mathematics 48 (2012), no. 5, 615-639.

[15] JM Hammersley, A few seedlings of research in proc. of the sixth berkeley symp. math. statist. and probability, vol. 1 (1972), 345-394, 1972.

[16] Christian Houdré and Ümit Işlak, A central limit theorem for the length of the longest common subsequence in random words, arXiv preprint arXiv:1408.1559 (2014).

[17] SV Kerov and AM Vershik, Asymptotics of the plancherel measure of the symmetric group and the limiting form of young tableaux, Soviet Math. Dokl, vol. 18, 1977, pp. 527-531.

[18] Benjamin F Logan and Larry A Shepp, A variational problem for random young tableaux, Advances in mathematics 26 (1977), no. 2, 206-222.

[19] George S Lueker, Improved bounds on the average length of longest common subsequences, Journal of the ACM (JACM) 56 (2009), no. 3, 17.

[20] Colin L Mallows, Non-null ranking models. i, Biometrika 44 (1957), no. 1/2, 114130.

[21] John I Marden, Analyzing and modeling rank data, volume 64 of monographs on statistics and applied probability, 1995.

[22] Carl Mueller and Shannon Starr, The length of the longest increasing subsequence of a random mallows permutation, Journal of Theoretical Probability 26 (2013), no. $2,514-540$.

[23] Pavel Pevzner, Computational molecular biology: an algorithmic approach, MIT press, 2000.

[24] H.L. Royden and P. Fitzpatrick, Real analysis, Prentice Hall, 2010.

[25] Richard P. Stanley, Enumerative combinatorics: Volume 1, 2nd ed., Cambridge University Press, New York, NY, USA, 2011.

[26] Shannon Starr, Thermodynamic limit for the mallows model on $s$, Journal of Mathematical Physics 50 (2009), no. 9, 095208.

[27] Volker Strassen, The existence of probability measures with given marginals, The Annals of Mathematical Statistics (1965), 423-439. 